Aus dem Institut für Pflanzenbau und Pflanzenzüchtung der Georg-August-Universität zu Göttingen

\title{
Wachstum und Ertrag von Getreide nach mechanischer Bodenbelastung in unterschiedlichen Bearbeitungssystemen
}

\author{
Dissertation \\ zur Erlangung des Doktorgrades \\ (Dr. sc. agr.) \\ der Fakultät für Agrarwissenschaften \\ der Georg-August-Universität Göttingen \\ vorgelegt von \\ Karl-Theodor Mähner \\ geboren in Hamm/Westfalen
}

Göttingen, Mai 1999

Gefördert durch die Deutsche Forschungsgemeinschaft 
D 7

Referent: $\quad$ Prof. Dr. W. Ehlers

Korreferent: $\quad$ Prof. Dr. M. Runge

Tag der mündlichen Prüfung: 08.07.1999

Diese Dissertation ist als elektronische Dissertation auf dem Dokumentenserver der Niedersächsischen Staats- und Universitätsbibliothek Göttingen im Internet veröffentlicht.

http://www.sub.uni-goettingen.de 
Inhaltsverzeichnis

Verzeichnis der Abkürzungen.............................................. 6

Verzeichnis der Abbildungen ................................................... 7

Verzeichnis der Tabellen ....................................................11

Kurzfassung ................................................................. 14

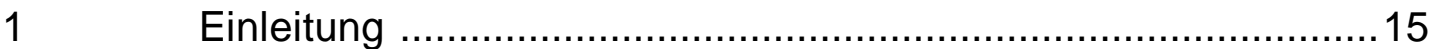

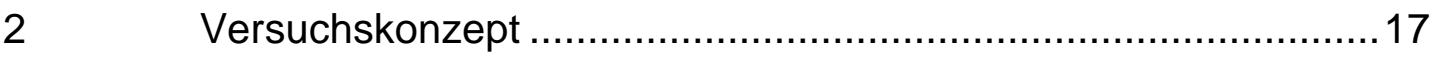

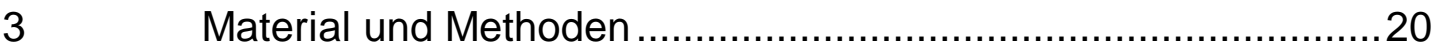

3.1 Versuchsanlage und Standortbeschreibung ..........................20

3.1.1 Einrichtung der mechanischen Bodenbelastung .......................20

3.1.2 Pflanzenbauliche Maßnahmen ............................................ 22

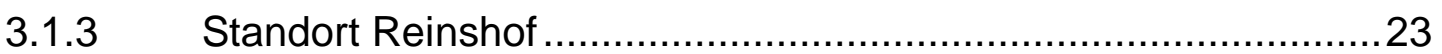

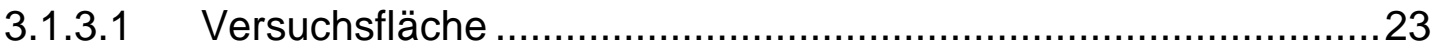

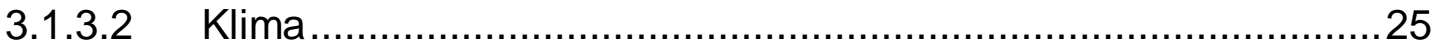

3.1.4 Standort Relliehausen ...................................................... 26

3.1.4.1 Versuchsfläche ................................................................. 26

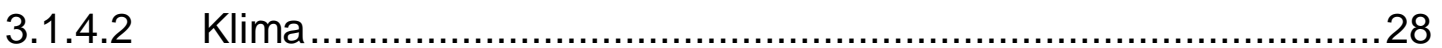

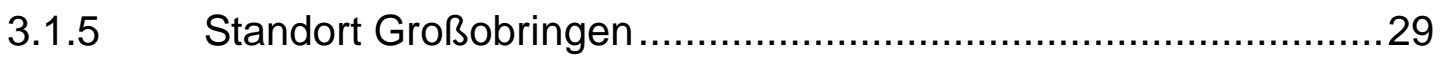

3.1.5.1 Versuchsfläche ...............................................................29

3.1.5.2 Klima ................................................................................. 31

3.2 Bodenphysikalische Untersuchungen.................................... 32

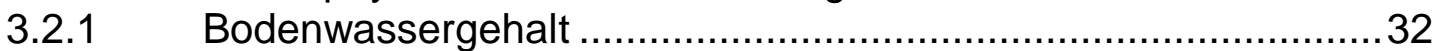

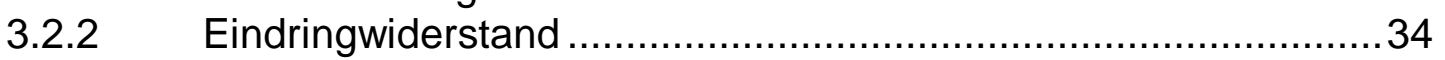

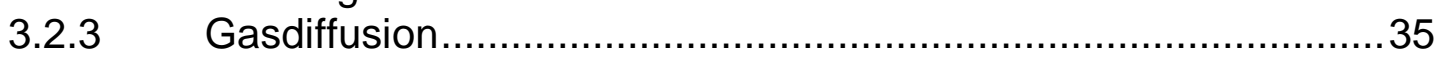

3.2.4 Ungesättigte Wasserleitfähigkeit ......................................... 36

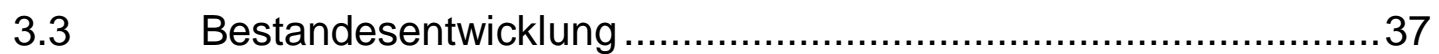

3.3.1 Pflanzendichte .................................................................. 37

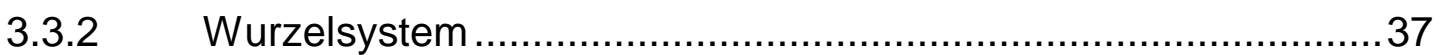

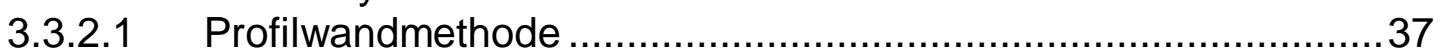

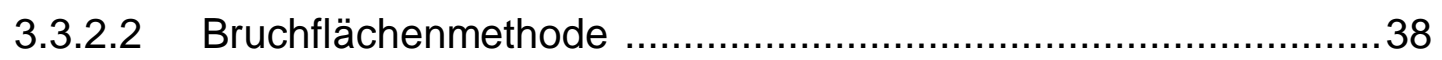

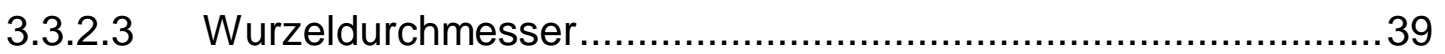

3.3.3 Oberirdische Trockenmasse .................................................. 40

3.3.4 Blattfläche .................................................................. 41

3.3.5 Bestandeshöhe ............................................................. 41

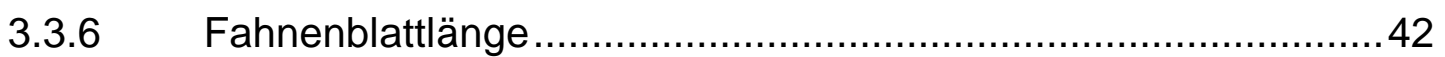

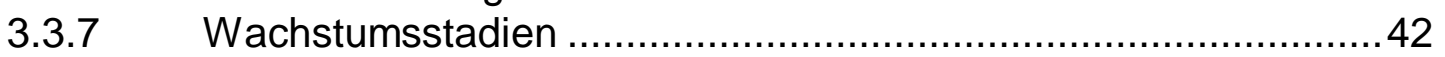

3.3.8 Gesamtwasserpotential .................................................... 42

3.3.9 Gaswechsel der Kulturpflanzen ............................................ 43

3.3.9.1 Ableitung von Stoffwechselkenngrößen ................................. 44

3.3.9.1.1 $\quad \mathrm{CO}_{2}-$ Assimlationsrate ..................................................... 44

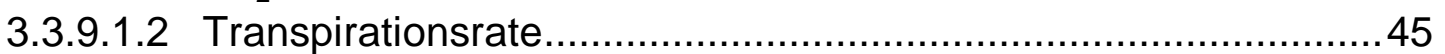

3.3.9.1.3 Stomatäre Leitfähigkeit für Wasserdampf............................. 45 
Seite

3.4 Untersuchungen von Ertragsbildung und Ertrag.......................45

3.4.1 Ährendichte und Kornbesatz der Ähre................................. 45

3.4.2 Korn und Strohertrag .................................................... 46

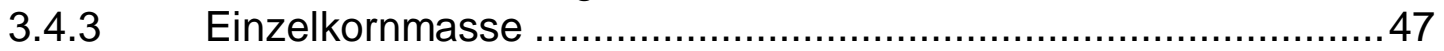

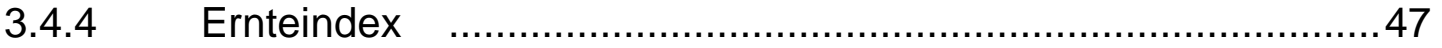

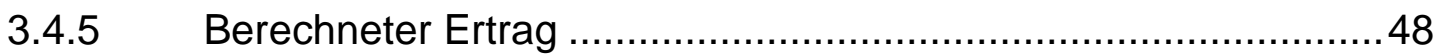

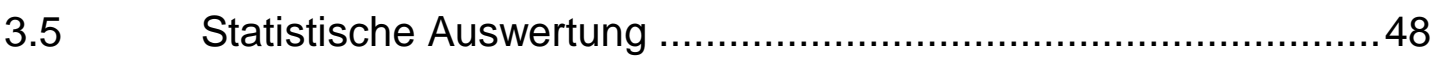

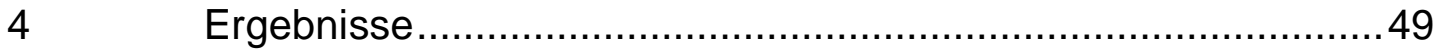

$4.1 \quad$ Bodenphysikalische Eigenschaften ..................................... 49

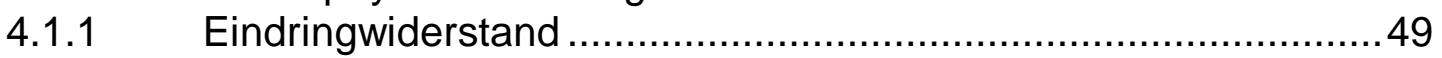

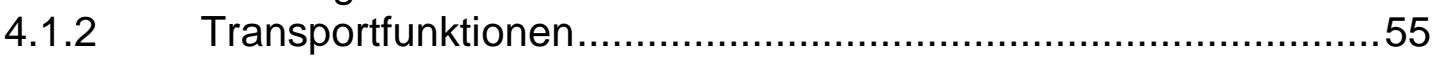

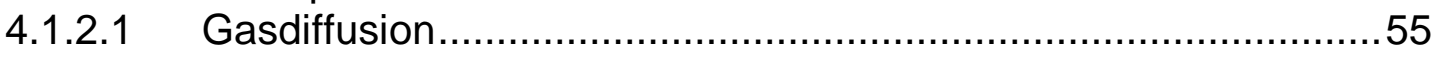

4.1.2.2 Ungesättigte Wasserleitfähigkeit .......................................61

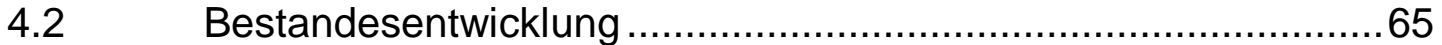

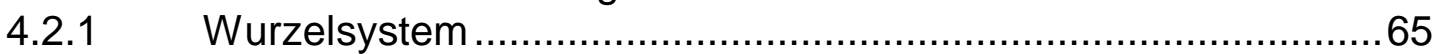

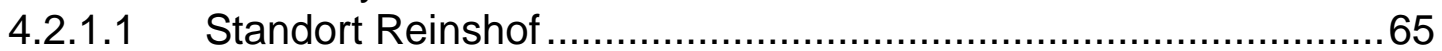

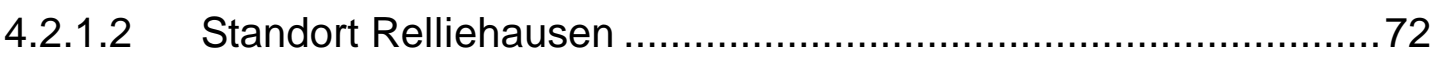

4.2.1.3 Standort Großobringen .................................................... 74

4.2.2 Oberirdische Trockenmasse und Blattfläche ...........................76

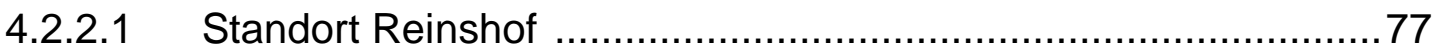

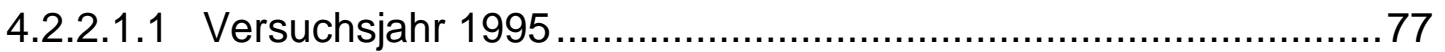

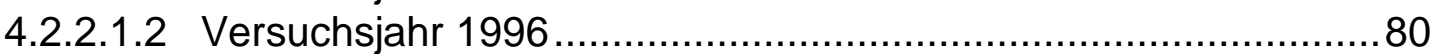

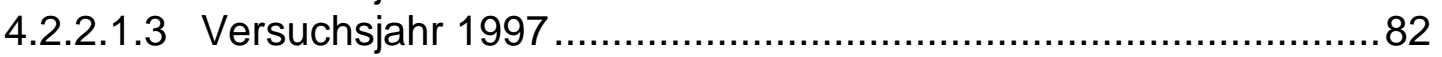

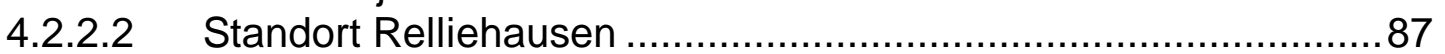

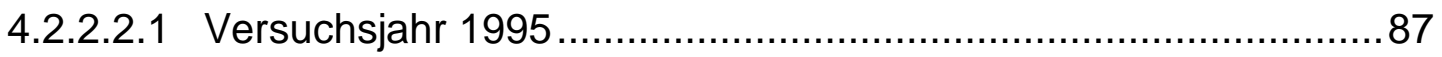

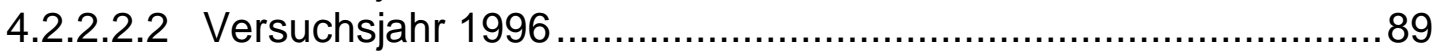

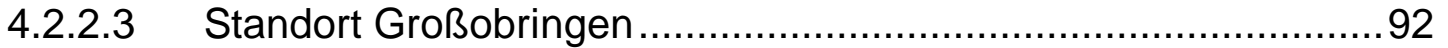

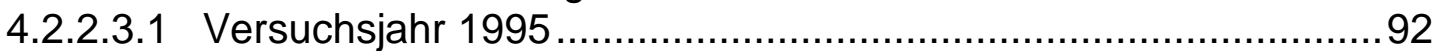

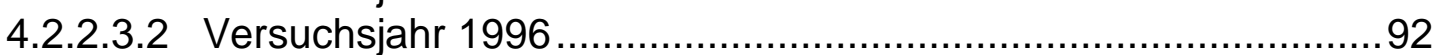

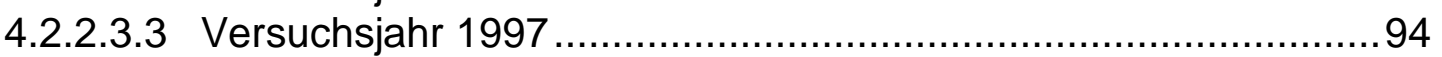

4.2.3 Gaswechsel und Gesamtwasserpotential ...............................96

4.3 Ertragsbildung und Ertrag................................................. 110

4.3.1 Standort Reinshof ........................................................... 111

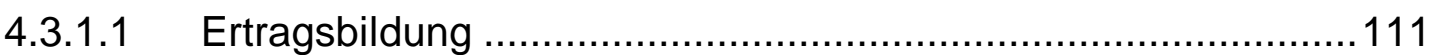

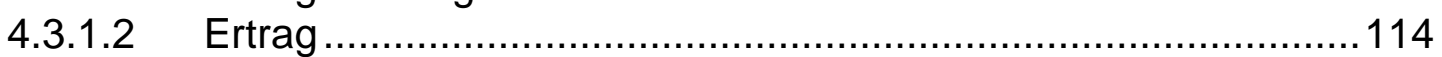

4.3.2 Standort Relliehausen ................................................... 116

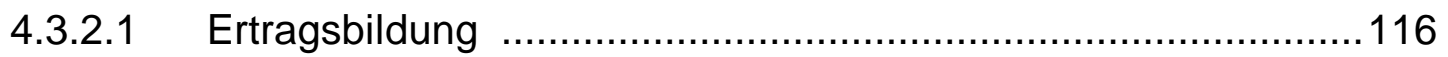

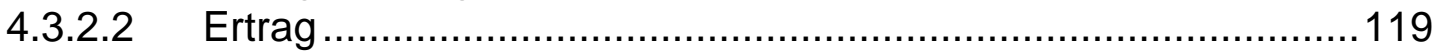

4.3.3 Standort Großobringen ............................................... 121

4.3.3.1 Ertragsbildung ............................................................ 121

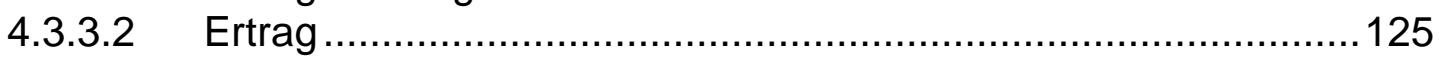


Seite

5.1 Auswirkungen der Bearbeitungssysteme und der mechanischen

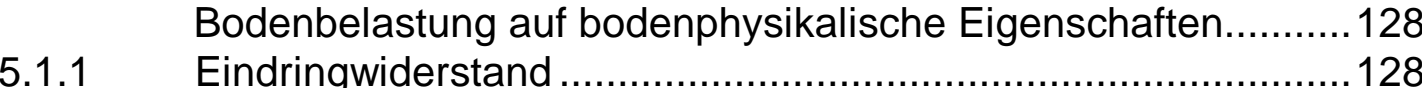

5.1.2 Transportfunktionen...................................................... 134

5.2 Auswirkungen der Bearbeitungssysteme und der mechanischen Bodenbelastung auf die Bestandesentwicklung .................... 140

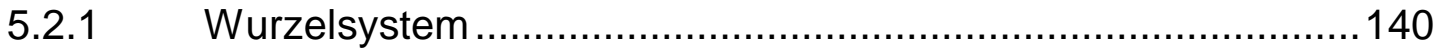

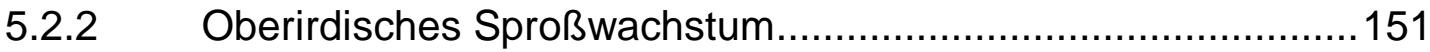

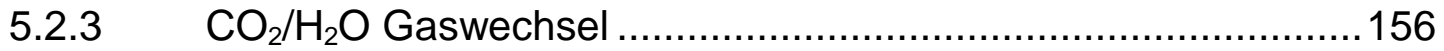

5.2.4 Ertragsbildung und Ertrag............................................. 160

Konsequenzen für die pflanzenbauliche Praxis......................166

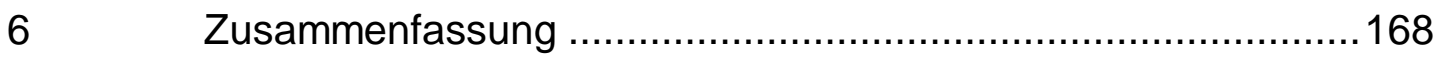

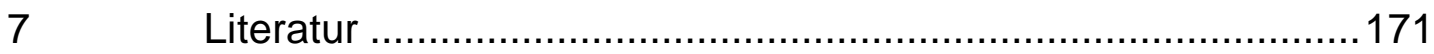

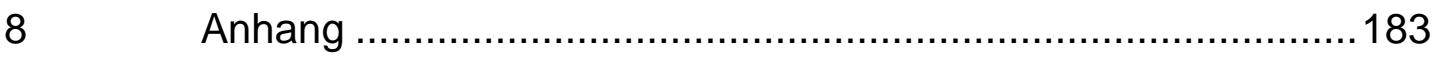


Verzeichnis der Abkürzungen und der verwendeten Einheiten

\begin{tabular}{|c|c|}
\hline$A G$ & Arbeitsgemeinschaft \\
\hline BFD & Blattflächendauer [Tage] \\
\hline $\mathrm{BFI}$ & Blattflächenindex [ - ] \\
\hline $\mathrm{Bl}$ & Block \\
\hline Bo & Bodenbearbeitungssystem \\
\hline Bs & Belastungsstufe \\
\hline $\mathrm{Bt}$ & Beprobungszeitpunkt [Tag des Jahres] \\
\hline Do & $\mathrm{O}_{2}$ - Gasdiffusionskoeffizient in Luft $\left[=0,203 \mathrm{~cm}^{2} / \mathrm{s}\right]$ \\
\hline Ds & scheinbarer Gasdiffusionskoeffizient $\left[\mathrm{cm}^{2} / \mathrm{s}\right]$ \\
\hline Ds/Do & relativer scheinbarer Gasdiffusionskoeffizient [-] \\
\hline EC & $\begin{array}{l}\text { Bezeichnung der Wachstumsstadien von Getreide } \\
\text { nach ZADOKS et al. } 1974\end{array}$ \\
\hline EDW & Eindringwiderstand [MPa] \\
\hline EKM & Einzelkornmasse $[\mathrm{mg}]$ \\
\hline El & luftgefülltes Porenvolumen $\left[\mathrm{cm}^{3} / \mathrm{cm}^{3}\right]$ \\
\hline FMW & Festboden-Mulchwirschaft \\
\hline GO & Standort Großobringen \\
\hline GWL & Gesamtwurzellänge $\left[\mathrm{km} / \mathrm{m}^{2}\right]$ \\
\hline GWP & Gesamtwasserpotential der Pflanze [bar] \\
\hline KBV & Krumenbasisverdichtung \\
\hline $\mathrm{ku}$ & ungesättigte hydraulische Wasserleitfähigkeit [cm/Tag] \\
\hline LBW & Lockerbodenwirtschaft \\
\hline LBW flach & Lockerbodenwirtschaft, flach bearbeitet \\
\hline LBW tief & Lockerbodenwirtschaft, tief bearbeitet \\
\hline LK & Luftkapazität [Vol. \%] \\
\hline $\mathrm{n}$ & Anzahl der Messungen pro Parzelle \\
\hline PAR & Photosynthetisch aktive Strahlung $\left[\mu \mathrm{mol}\right.$ Photonen $\left./ \mathrm{m}^{2} / \mathrm{s}\right]$ \\
\hline $\mathrm{pF}$ & $\begin{array}{l}\text { dekadischer Logarithmus der Wasserspannung } \\
\text { in cm Wassersäule }\end{array}$ \\
\hline $\mathrm{RH}$ & Standort Reinshof \\
\hline $\mathrm{RL}$ & Standort Relliehausen \\
\hline SG & Sommergerste \\
\hline $\mathrm{T}$ & Tiefe der untersuchten Bodenschicht $[\mathrm{cm}]$ \\
\hline TM & Trockenmasse $[\mathrm{g}]$ \\
\hline WG & Wintergerste \\
\hline WLD & Wurzellängendichte $\left[\mathrm{cm} / \mathrm{cm}^{3}\right]$ \\
\hline WW & Winterweizen \\
\hline
\end{tabular}


Abb. 1: Versuchsplan Standort Reinshof

Abb. 2: Niederschläge (Monatssummen) und Lufttemperatur (Monatsmittel) als langjähriges Mittel (1951-1980) und in den Jahren 1995, 1996 und 1997 auf dem Standort Reinshof .................................... 25

Abb. 3: Versuchsplan Standort Relliehausen ........................................... 27

Abb. 4: Niederschläge (Monatssummen) als langjähriges Mittel (19691997) und in den Versuchsjahren 1995, 1996 und 1997 sowie die Lufttemperatur in Relliehausen ................................................. 28

Abb. 5: Versuchsplan Standort Großobringen ........................................... 30

Abb. 6: Niederschläge (Monatssummen) und Lufttemperatur (Monatsmittel) als langjähriges Mittel (1983-1997) und in den Jahren 1995, 1996 und 1997 auf dem Standort Großobringen.

Abb. 7: Eindringwiderstand - Tiefenfunktionen des Standortes Reinshof der Jahre 1995,1996 und 1997

Abb. 8: Eindringwiderstand - Tiefenfunktionen des Standortes Relliehausen der Jahre 1996 und 1997

Abb. 9: Eindringwiderstand - Tiefenfunktionen des Standortes Großobringen der Jahre 1996 und 1997.

Abb. 10: Beziehung zwischen Ds/Do und dem luftgefültten Porenvolumen (EI) in Reinshof am12.04.1995 in 28-34 cm Bodentiefe....

Abb. 11: Beziehung zwischen Ds/Do und dem luftgefüllten Porenvolumen (EI) in Relliehausen am 26.04.1995 in 28-34 cm Bodentiefe 58

Abb. 12: Beziehung zwischen Ds/Do und dem luftgefüllten Porenvolumen

(El) in Großobringen am 24.03.1995 in 28-34 cm Bodentiefe 59

Abb. 13: Beziehung zwischen Ds/Do und dem luftgefültten Porenvolumen (EI) in Reinshof am16.04.1997 in 28-34 cm Bodentiefe.

Abb. 14: Beziehung zwischen der ungesättigten hydraulischen Leitfähigkeit (ku) und der Wasserspannung des Bodens in der Bodentiefe 28$34 \mathrm{~cm}$ am 12.04.1995 auf dem Standort Reinshof.

Abb. 15: Beziehung zwischen der ungesättigten hydraulischen Leitfähigkeit (ku) und der Wasserspannung des Bodens in der Bodentiefe 28$34 \mathrm{~cm}$ am 26.04.1995 auf dem Standort Relliehausen.

Abb. 16: Beziehung zwischen der ungesättigten hydraulischen Leitfähigkeit (ku) und der Wasserspannung des Bodens in der Bodentiefe 28$34 \mathrm{~cm}$ am 24.03.1995 auf dem Standort Großobringen ....

Abb. 17: Wurzelprofil für Sommergerste nach der Profilwandmethode im Versuchsjahr 1995 auf dem Standort Reinshof

Abb. 18: Wurzelprofil für Sommergerste nach der Profilwandmethode im Versuchsjahr 1997 auf dem Standort Reinshof

Abb. 19: Gesamtwurzellänge auf dem Standort Reinshof.

Abb. 20: Wirkung der mechanischen Bodenbelastung und des Bearbeitungssystems auf den Wurzeldurchmesser von Winterweizen auf dem Standort Reinshof 1996

Abb. 21: Gesamtwurzellänge (GWL) und GWL in Oberboden (0-30 cm Bodentiefe, weiße Säulen) und Unterboden (von $30 \mathrm{~cm}$ Bodentiefe maximale Durchwurzelungstiefe, schwarze Säulen) auf dem Standort Relliehausen 
Seite

Abb. 22: Gesamtwurzellänge (GWL) und GWL in Oberboden $(0-30 \mathrm{~cm}$ Bodentiefe, weiße Säulen) und Unterboden (von $30 \mathrm{~cm}$ Bodentiefe $50 \mathrm{~cm}$ Durchwurzelungstiefe, schwarze Säulen) auf dem Standort Großobringen .

Abb. 23: Entwicklung der oberirdischen Trockenmasse (oben) und des Blattflächenindexes (unten) von Sommergerste über die Zeit auf dem Standort Reinshof im Versuchsjahr 1995.

Abb. 24: Bestandeshöhe der Sommergerste Reinshof 1995

Abb. 25: Entwicklung der oberirdischen Trockenmasse (oben) und des Blattflächenindexes (unten) von Winterweizen über die Zeit auf dem Standort Reinshof im Versuchsjahr 1996.

Abb. 26: Blattflächendauer (BFD) des Winterweizens vom Ende der Blüte (EC 69) bis zur mittleren Milchreife (EC 75) auf dem Standort

Reinshof 1996 in Abhängigkeit von der Belastungsstufe

Abb. 27: Bestandeshöhe des Winterweizens Reinshof 1996.

Abb. 28: Entwicklung der oberirdischen Trockenmasse (oben) und des Blattflächenindexes (unten) von Wintergerste auf dem Standort Reinshof über die Zeit im Versuchsjahr 1997.

Abb. 29: Blattflächendauer (BFD) der Wintergerste vom Ende der Blüte (EC 69) bis zur Gelbreife (EC 87) auf dem Standort Reinshof 1997 in Abhängigkeit von der Belastungsstufe...

Abb. 30: Bestandeshöhe der Wintergerste Reinshof 1997

Abb. 31: Entwicklung der oberirdischen Trockenmasse (oben) und des Blatt-flächenindexes (unten) von Sommergerste auf dem Standort

Relliehausen über die Zeit im Versuchsjahr 1995

Abb. 32: Entwicklung der oberirdischen Trockenmasse (oben) und des Blattflächenindexes (unten) von Winterweizen auf dem Standort Relliehausen über die Zeit im Versuchsjahr 1996

Abb. 33: Blattflächendauer (BFD) des Winterweizens vom Ende der Blüte (EC 69) bis zur mittleren Milchreife (EC 75) auf dem Standort Relliehausen 1996 in Abhängigkeit von der Belastungsstufe.

Abb. 34: Bestandeshöhe des Winterweizens auf dem Standort Relliehausen 1996

Abb. 35: Entwicklung der oberirdischen Trockenmasse (oben) und des Blattflächenindexes (unten) von Winterweizen über die Zeit auf dem Standort Großobringen im Versuchsjahr 1996.

Abb. 36: Entwicklung der oberirdischen Trockenmasse (oben) und des Blattflächenindexes (unten) von Wintergerste über die Zeit auf dem Standort Großobringen im Versuchsjahr 1997.

Abb. 37: Gang der photosynthetisch aktiven Strahlung (PAR) und des Sättigungsdefizites am 23.06.1996 auf dem Standort Reinshof.

Abb. 38: Stomatäre Leitfähigkeit (oben) und $\mathrm{CO}_{2}$-Assimilationsrate des Winterweizens (unten) gemessen am Fahnenblatt auf dem Standort Reinshof als Funktion der Zeit am 23.06.1996

Abb. 39: Gesamtwasserpotential im Winterweizen als Funktion der Zeit am 23.06.1996 auf dem Standort Reinshof 
Seite

Abb. 40: Bodenwassergehalt als Funktion der Bodentiefe auf dem Standort Reinshof am 23.06.1996.

Abb. 41: Die $\mathrm{CO}_{2}$-Assimilationsrate von Winterweizen (gemessen am Fahnenblatt) als Funktion der photosynthetisch aktiven Strahlung (PAR) zum Stadium EC 69 bei Lockerbodenwirtschaft (LBW) auf dem Standort Reinshof vom 23.06.1996

Abb. 42: Lichtfluß-Effektkurven des Winterweizens am 23.06.1996 im Sta-

dium EC 69 auf dem Standort Reinshof

Abb. 43: Gang der photosynthetisch aktiven Strahlung (PAR) und des Sättigungsdefizites am 18.06.1997 und am 19.06.1997 auf dem Standort Reinshof.

Abb. 44: Stomatäre Leitfähigkeit (oben) und $\mathrm{CO}_{2}$-Assimilationsrate des Wintergerste (unten) gemessen am Fahnenblatt auf dem Standort

Reinshof als Funktion der Zeit am 18. und 19. 06.1997...

Abb. 45:Gesamtwasserpotential in der Wintergerste als Funktion der Zeit am 18. und 19.06.1996 auf dem Standort Reinshof

Abb. 46: Bodenwasserausschöpfung vom 04.06.1997 bis zum 19.06.1997 aus einzelnen Bodenschichten auf dem Standort Reinshof..

Abb. 47: Lichtfluß-Effektkurven der Wintergerste zum Stadium EC 69 bis 83 imJahr 1997 auf dem Standort Reinshof.

Abb. 48: Kornertrag auf dem Standort Reinshof ....

Abb. 49: Kornertrag auf dem Standort Relliehausen.

Abb. 50: Kornertrag auf dem Standort Großobringen.

Abb. 51: Beziehung zwischen der Lagerungsdichte des Bodens und dem Eindringwiderstand (EDW) in der Bodentiefe $30-35 \mathrm{~cm}$ auf den Standort Reinshof

Abb. 52: Bodentemperatur in zwei Bodentiefen, gemessen an der Wetterstation Göttingen.

Abb. 53: Relativer scheinbarer Gasdiffusionskoeffizient bei einer Wasserspannung von pF 2,0 als Funktion der Lagerungsdichte des Bodens in der Tiefe 28-34 cm auf dem Standort Reinshof in den Versuchsjahren 1995 und 1997

Abb. 54: Relativer scheinbarer Gasdiffusionskoeffizient bei einer Wasserspannung von pF 2,0 in der Bodentiefe 28-34 cm in Abhängigkeit von der Belastungsstufe im Versuchsjahr 1995.

Abb. 55: Relativer scheinbarer Gasdiffusionskoeffizient bei einer Wasserspannung von pF 2,0 in der Bodentiefe $28-34 \mathrm{~cm}$ in Abhängigkeit von der Belastungsstufe in den Versuchsjahren 1995 und 1997 auf dem Standort Reinshof.

Abb. 56: Beitrag der Poren von 60 bis $300 \mu$ m Äquivalentdurchmesser an der ungesättigten hydraulischen Leitfähigkeit in Abhängigkeit von der Belastungsstufe auf dem Standort Reinshof in der Bodentiefe 28-34 cm am 12.04.1995

Abb. 57: Gesamtwurzellänge (GWL) unterhalb $35 \mathrm{~cm}$ Bodentiefe am 03.06.1995 als Funktion des Eindringwiderstandes in der Tiefe 30$35 \mathrm{~cm}$ am 04.04.1995 und des relativen scheinbaren Gasdiffusionskoeffizienten am 12.04.1995 auf dem Standort Reinshof 
Seite

Abb. 58: Beziehung des relativen scheinbaren Gasdiffusionskoeffizienten bei pF 2,0 zur Luftkapazität ( $p F 1,8$ ) bei steigender Bodenbelastung in der Bodenschicht 28-34 cm auf dem Standort Reinshof am 12.04.1995 und am 16.04.1997

Abb. 59: Veränderung der Gesamtwurzellänge im Unterboden auf dem Standort Reinshof 1995

Abb. 60:Gesamtwurzellänge unterhalb $35 \mathrm{~cm}$ Bodentiefe am 02.06.1997 als Funktion des Eindringwiderstandes in der Tiefe $30-35 \mathrm{~cm}$ am 19.03.1997 und des relativen scheinbaren Gasdiffusionskoeffizienten bei pF 2,0 in der Tiefe 28-34 cm am 16.04.1997 auf dem Standort Reinshof....

Abb. 61: Beziehung zwischen der Gesamtwurzellänge am 03.07.1995 (EC 45) und der oberirdischen Trockenmasse der Sommergerste am 23.06.1995 (EC37) auf dem Standort Reinshof

Abb. 62: Beziehung zwischen der Gesamtwurzellänge im Unterboden am 02.06.1997 und der mittleren stomatären Leitfähigkeit am 18. und 19.06.1997 auf dem Standort Reinshof

Abb. 63: Gemessener Kornertrag der Sommergerste 1995 als Funktion der Gesamtwurzellänge im Unterboden auf den Standorten Reinshof, Relliehausen und Großobringen

Abb. 64: Beziehung zwischen dem Kornertrag und dem mittlerer Blattflächenindex von EC 25-37 der Sommergerste auf dem Standort Reinshof im Versuchsjahr 1995

Abb. 65: Beziehung zwischen gemessenem und berechnetem Kornertrag auf den Standorte Reinshof, Relliehausen und Großobringen in den Jahren 1996 und 1997. 
Verzeichnis der Tabellen

Seite

Tab. 1: Kennzeichnung der Belastungsstufen. 20

Tab. 2: Termine der mechanischen Belastung. 21

Tab. 3: Bodenwassergehalt (BWG) und Feldkapazität (FK) der Böden bei der mechanischen Belastung zu Versuchsbeginn auf den Standorten Reinshof und Relliehausen

Tab. 4: Bodenwassergehalt (BWG) und Feldkapazität (FK) der Böden bei der mechanischen Belastung zu Versuchsbeginn auf dem Standort Großobringen.

Tab. 5: Horizontfolge und Korngrößenzusammensetzung des Bodens auf der Versuchsfläche Reinshof.

Tab. 6: Horizontfolge und Korngrößenzusammensetzung des Bodens auf der Versuchsfläche Relliehausen

Tab. 7: Horizontabfolge und Korngrößenzusammensetzung des Bodens auf der Versuchsfläche Großobringen

Tab. 8: Beprobungsplan Bodenwassergehalt auf den Standorten Reins-

hof (RH), Relliehausen (RL) und Großobringen (GO)

Tab. 9: Beprobungszeitpunkte (Bt) zum Bodenwassergehalt auf den Standorten Reinshof (RH), Relliehausen (RL) und Großobrin-

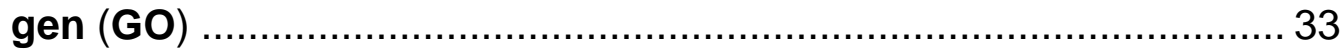

Tab. 10: Beprobungsplan Eindringwiderstand ........................................... 34

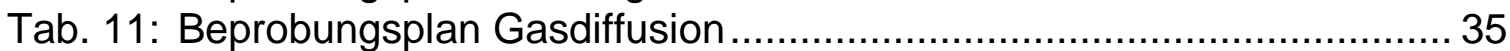

Tab. 12: Beprobungsplan ungesättigte Wasserleitfähigkeit ............................. 36

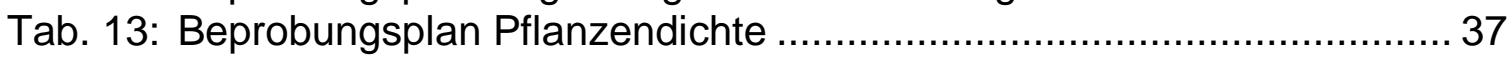

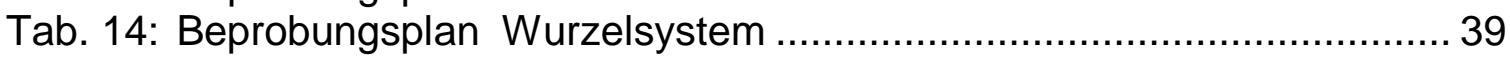

Tab. 15: Beprobungstermine der Teilernten zur Bestimmung der oberirdischen Biomasse und der Blattfläche .............................................. 40

Tab. 16: Beprobungsplan oberirdische Biomasse und Blattfläche ....................41 4

Tab. 17: Beprobungsplan Bestandeshöhe ................................................. 42

Tab. 18: Beprobungsplan Fahnenblattlänge ................................................. 42

Tab. 19: Beprobungsplan Gesamtwasserpotential ....................................... 43

Tab. 20: Beprobungsplan $\mathrm{CO}_{2} / \mathrm{H}_{2} \mathrm{O}$ - Gaswechsel .................................... 44

Tab. 21: Beprobungsplan Ährendichte und Kornbesatz der Ähre ..................... 46

Tab. 22: Beprobungsplan Korn- und Strohertrag ...................................... 47

Tab. 23: Bestandeshöhe der Sommergerste zum Zeitpunkt der Ernte im Versuchsjahr 1995 auf dem Standort Großobringen...................... 92

Tab. 24: Bestandeshöhe des Winterweizens zum Zeitpunkt der Ernte im Versuchsjahr 1996 auf dem Standort Großobringen...................... 93

Tab. 25: Bestandeshöhe der Wintergerste zum Zeitpunkt der Ernte im Versuchsjahr 1997 auf dem Standort Großobringen

Tab. 26: Ertragsbildung bei Sommergerste im Jahr 1995 auf dem Standort Reinshof. EKM bezeichnet die Einzelkornmasse.

Tab. 27: Ertragsbildung bei Winterweizen im Jahr 1996 auf dem Standort Reinshof.

Tab. 28: Ertragsbildung bei Wintergerste im Jahr 1997 auf dem Standort Reinshof.

Tab. 29: Ernteindex bei Sommergerste (SG) 1995, Winterweizen (WW) 1996 und Wintergerste (WG) 1997 auf dem Standort Reinshof 
Seite

Tab. 30: Ertragsbildung bei Sommergerste im Jahr 1995 auf dem Standort Relliehausen.

Tab. 31: Ertragsbildung bei Winterweizen im Jahr 1996 auf dem Standort Relliehausen.

Tab. 32: Ernteindex bei Sommergerste (SG) 1995, Winterweizen (WW) 1996 und Wintergerste (WG) 1997 auf dem Standort Relliehausen.

Tab. 33: Ertragsbildung der Sommergerste im Jahr 1995 auf dem Standort Großobringen

Tab. 34: Ertragsbildung bei Winterweizen im Jahr 1996 auf dem Standort Großobringen

Tab. 35: Ertragsbildung bei Wintergerste im Jahr 1997 auf dem Standort Großobringen

Tab. 36: Ernteindex bei Sommergerste (SG) 1995, Winterweizen (WW) 1996 und Wintergerste (WG) 1997 auf dem Standort Großobringen

Tab. 37: Bodenwassergehalt auf den Standorten Reinshof (20.07.1995) und Relliehausen (05.07.1995)

Anhang

Tab. 38: Technische Daten und Gewichte der verwendeten Fahrzeuge ......... 183

Tab. 39: Kontaktflächendruck bei den Belastungsstufen .............................. 183

Tab. 40: Einteilung der Entwicklungsstadien nach ZADOKS et al. 1974 ............ 184

Tab. 41: Lagerungsdichte und Luftkapazität bei pF 1,8 auf dem Standort Reinshof 1995.

Tab. 42: Lagerungsdichte und Luftkapazität bei pF 1,8 auf dem Standort Reinshof 1996.

Tab. 43: Lagerungsdichte und Luftkapazität bei pF 1,8 auf dem Standort Reinshof 1997.

Tab. 44: Lagerungsdichte und Luftkapazität bei pF 1,8 auf dem Standort Relliehausen 1995.

Tab. 45: Lagerungsdichte und Luftkapazität bei pF 1,8 auf dem Standort Relliehausen 1996.

Tab. 46: Lagerungsdichte und Luftkapazität bei pF 1,8 auf dem Standort Relliehausen 1997.

Tab. 47: Lagerungsdichte und Luftkapazität bei pF 1,8 auf dem Standort Großobringen 1995

Tab. 48: Lagerungsdichte und Luftkapazität bei pF 1,8 auf dem Standort Großobringen 1996 .

Tab. 49: Lagerungsdichte und Luftkapazität bei pF 1,8 auf dem Standort Großobringen 1997 .

Tab. 50: Wurzellängendichte der Sommergerste in der Bodenmatrix (idBm) und in Bioporen (iBp)auf dem Standort Reinshof am 03.07.1995.... 195

Tab. 51: Wurzellängendichte der Sommergerste in der Bodenmatrix (idBm) und in Bioporen (iBp)auf dem Standort Reinshof am 26.07.1995 .... 196

Tab. 52: Wurzellängendichte des Winterweizens auf dem Standort Reinshof am 15.05.1996 
Tab. 53: Wurzellängendichte des Winterweizens auf dem Standort Reinshof am 12.06.1996 ........................................................... 198

Tab. 54: Wurzellängendichte des Winterweizens auf dem Standort Reins-

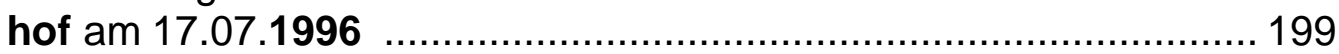

Tab. 55: Wurzellängendichte der Wintergerste auf dem Standort Reinshof am 14.05.1997

Tab. 56: Wurzellängendichte der Wintergerste auf dem Standort Reinshof am 02.06.1997

Tab. 57: Pflanzenbauliche Maßnahmen auf dem Standort Reinshof in den Jahren 1995-1997.

Tab. 58: Pflanzenbauliche Maßnahmen auf dem Standort Relliehausen in den Jahren 1995-1997

Tab. 59: Pflanzenbauliche Maßnahmen auf dem Standort Großobringen in den Jahren 1995-1997 
Kurzfassung

Vor dem Hintergrund einer ständig steigenden Belastung der Ackerböden durch schwere Maschinen weisen praktische Erfahrungen darauf hin, daß Böden mit

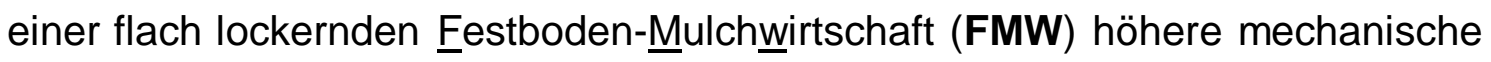
Belastbarkeit und ein höheres Regenerationsvermögen besitzen können als Böden mit tief-wendender Lockerbodenwwirtschaft (LBW). Um diese These zu prüfen, wurden Lößböden an drei Standorten mit den Bearbeitungssystemen LBW und FMW im Frühjahr 1995 bei Feldkapazität einer einmaligen geringen, mittleren oder hohen mechanischen Belastung ausgesetzt. Im Feld und Labor wurden nach dem Leitgedanken der Wirkkette mechanische Belastung $\rightarrow$ Bodeneigenschaften $\rightarrow$ Wurzelwachstum $\rightarrow$ Sproßwachstum $\rightarrow$ Ertrag bodenphysikalische Kennwerte, Wurzel- und Sproßwachstum, Gaswechsel der Pflanzen sowie der Korn- und Strohertrag erfaßt.

Die Beeinträchtigung bodenphysikalischer Eigenschaften wie Lagerungsdichte, Porenraumgestaltung und Eindringwiderstand führte im Anlagejahr bei beiden Bearbeitungssystemen zu einer Reduktion von Wurzel- und Sproßwachstum, bei LBW viel stärker ausgeprägt als bei FMW. Im Anlagejahr führte steigende Belastung auf LBW zu Ertragsdepressionen der Sommergerste, die auch bei FMW, aber beschränkt auf mittlere und hohe Belastung, nachweisbar waren.

Im zweiten und dritten Versuchsjahr mit Winterweizen und Wintergerste fiel der Ertragsrückgang - wenn überhaupt feststellbar - geringer aus als im Anlagejahr. In der Tendenz war er bei LBW stärker ausgeprägt als bei FMW. In Trokkenperioden wurde bei LBW durch Behinderung des Wurzelwachstums nach hohem Lasteintrag ein Wasserstreß in den Pflanzen ausgelöst, der den Gaswechsel am Fahnenblatt erniedrigte.

In den drei Versuchsjahren beschränkte sich die Regeneration des Bodens auf die Ausbildung von Rissen und Bioporen. Die Untersuchungen lassen den Schluß zu, daß Lößböden mit FMW im Vergleich zu LBW eine höhere mechanische Belastbarkeit aufweisen und sich durch ein höheres Regenerationsvermögen auszeichnen. 
Niemals noch hat Unwissenheit jemandem genützt. Karl Marx 1867

\section{Einleitung}

Die aktuelle landwirtschaftliche Praxis des Pflanzenbaus steht im Spannungsfeld von Ökonomie und Ökologie (DEWIT 1991, BAEUMER 1992, DAHL 1997). Oft scheinen die Ziele der Produktion unvereinbar: Stabile Höchsterträge müssen mit größtmöglicher Umweltverträglichkeit der angewandten Verfahrenstechnik verknüpft werden, wenn die Ertragsfähigkeit als Teil der ökologischen Funktion der Ackerböden langfristig erhalten bleiben soll. Während der Gesetzgeber in einigen Bereichen der Pflanzenproduktion wie zum Beispiel dem chemischen Pflanzenschutz ein umfangreiches Regelwerk einsetzt, verläßt er sich beim Bodenschutz auf die gute fachliche Praxis (GESETZ ZUM SCHUTZ DES BODENS DER BUNDESREPUBLIK DEUTSCHLAND 1998). Betrachtet man die heutige "gute fachliche Praxis" so zeigt sich, daß der Wendepflug als Leitgerät einer Lockerbodenwirtschaft (LBW), einen wesentlichen Bestandteil der Bodenbearbeitung darstellt. Die Gründe sind leicht aufgeführt: Der Wendepflug vergräbt Erntereste ebenso gründlich wie Konkurrenzpflanzen einschließlich deren Samen und hinterläßt einen „sauberen“ Acker. Je nach Arbeitstiefe ist die Krume aufgelokkert und bietet günstige Voraussetzungen für das Saatbett der nachfolgenden Feldfrucht. Den Vorteilen des Wendepfluges stehen seine Nachteile gegenüber: Der Einsatz von Zeit und Energie bei der Pflugarbeit ist hoch. Neben der Zunahme von Erosionsschäden können Verdichtungen und Strukturschäden in der Krumenbasis - einer Bodenschicht direkt unterhalb des Bearbeitungshorizontes - und im Unterboden Folge des Pflügens sein. Die immer größer werdende Belastung der gepflügten Ackerböden durch zunehmend schwerere Landmaschinen führte dazu, daß auf ca. 50 \% der landwirtschaftlichen Nutzfläche der alten Bundesländer mit Schadverdichtungen bis in den Unterboden gerechnet werden muß (RUHM 1983). Unter einer Bodenverdichtung soll im folgenden eine Gefügeveränderung, verbunden mit der Zunahme der Lagerungsdichte des Bodens und der Abnahme des Porenvolumens verstanden werden. Eine Schadverdichtung liegt vor, wenn die Gefügeveränderung das Wachstum 
der Kulturpflanzen behindert (BRANDHUBER 1997). In zahlreichen Untersuchungen wurde auf die Gefahr einer dauerhaften Schädigung des Unterbodens gepflügter Böden durch eine hohe mechanische Belastung hingewiesen (HÅkANSSON et al. 1987, VoORHEEs et al. 1989, PETELKAU \& DANNOWSKI 1990, Domzal et al. 1991, LHOTSKY et al. 1991, LOWERY \& SCHULER 1991, RUSANOW 1991, Gameda et al. 1994, Hammel 1994, Riley 1994, AlakUKKu \& Elonen 1994, ETANA \& HÅKANSSON 1994, SCHJØNNING \& RASMUSSEN 1994, AlBLAS et al. 1994, SOMmeR et al. 1995). In der pflanzenbaulichen Praxis der letzten Jahrzehnte haben Züchtungsfortschritte und eine Optimierung des Pflanzenschutzund Düngermanagements die zunehmende Schädigung des Bodengefüges oft durch eine Stabilisierung oder Steigerung der Erträge überlagert und damit unkenntlich gemacht (DüRR et al. 1995). Eine fortschreitende mechanische Belastung der Böden unter Pflug verbunden mit einer zunehmenden Einschränkung der ökologischen Funktionen des Unterbodens durch Gefügedeformationen können die Ertragssicherheit auf lange Sicht jedoch erheblich gefährden.

Mit dem Ziel einer bodenschonenden Landbewirtschaftung können Bodenbearbeitungssysteme mit reduziertem mechanischen Bodeneingriff und einer Mulchdecke zum Schutz der Bodenoberfläche eine mögliche Alternative zum Wendepflug darstellen (EHLERS 1991). Solche Bearbeitungssysteme mit dauerhaftem Pflugverzicht können als Eestboden-Mulchwirtschaft (FMW) beschrieben werden (BAEUMER 1992). Die anhaltende Bodenruhe in Verbindung mit einer im Vergleich zu gepflügten Böden erhöhten biologischen Aktivität (STOCKFISCH et al. 1995, LANGMAACK et al. 1997) kann auf reduziert bearbeiteten Böden zu einem kontinuierlichen, anisotropen Porensystem führen, daß Ober - und Unterboden miteinander verbindet (EHLERS 1977, BALL 1981). Ein solches Porensystem kann sich durch eine hohe Stabilität gegenüber einer vertikal angreifenden Last auszeichnen (HARTGE \& BOHNE 1983, BLACKWELL et al.1990). Neben der Veränderung des Porensystems kann es auf reduziert bearbeiteten Böden zu einem Anstieg der Aggregatstabilität (EHLERS 1997) und der Aggregatporosität (VOORHEES \& LINDSTROM 1984 kommen. Als Ursache für die Veränderung der Aggregateigenschaften bei reduzierter Bodenbearbeitung kann die oberflächennahe Anreicherung mit organischer Substanz, die hohe biologische Aktivität (EHLERS 1997) und die anhaltende Bodenruhe bei fortlaufenden Quellungs- sowie Schrumpfungsprozessen des Bodens im Jahresgang 
(HORN et al. 1997) angeführt werden. Eine Reihe von Untersuchungen hat gezeigt, daß die höhere Stabilität der Porensysteme und der Bodenaggregate bei langfristig reduzierter Bodenbearbeitung zu einer größeren Unempfindlichkeit solcher Böden gegenüber mechanischer Belastung führen kann (HORN 1986, DeXTer 1988, Baumgartl 1991, Diez 1991, EhLERS et al. 1994, Gruber 1994).

Sollten in der kommenden Zeit die Bestimmungen des Gesetzes zum Schutze des Bodens umgesetzt werden, wird die Grundbodenbearbeitung mit dem Wendepflug unter dem Aspekt der bodenschonenden, standortangepaßten Bodenbearbeitung neu zu beurteilen sein. Es ist dringend erforderlich, alternative Bodenbearbeitungssysteme hinsichtlich ihrer Praxistauglichkeit sowie ihrer ökonomischen und ökologischen Vor- und Nachteile zu prüfen. In der vorliegenden Arbeit wurden die Auswirkungen mechanischer Bodenbelastung auf bodenphysikalische Eigenschaften und Merkmale des Pflanzenwachstums bei den kontrastierenden Bearbeitungssystemen LBW und FMW untersucht. Die Wirkkette: mechanische Belastung $\rightarrow$ Bodeneigenschaften $\rightarrow$ Wurzelwachstum $\rightarrow$ Sproßwachstum $\rightarrow$ Ertrag diente dabei als Leitfaden.

\section{Versuchskonzept}

An drei Standorten in Niedersachsen und Thüringen mit lößbürtigen Böden aber unterschiedlichem Niederschlagsregime wurde die Wirkung einer einmaligen mechanischen Bodenbelastung auf Bodeneigenschaften, Bodenleben und Pflanzenwachstum geprüft. Der Vergleich der Böden mit LBW und FMW hinsichtlich ihrer Empfindlichkeit gegen mechanische Belastung stand im Vordergrund.

Folgende Forschungsschwerpunkte wurden von vier Arbeitsgruppen (AG) bearbeitet: Universität Göttingen, Institut für Pflanzenbau und Pflanzenzüchtung: Wirkung mechanischer Belastung auf Gefügefunktionen und Pflanzenwachstum. Thüringer Landesanstalt für Landwirtschaft (TLL), Jena: Gefügeentwicklung nach mechanischer Belastung. Universität Kiel, Institut für Pflanzenernährung und Bodenkunde: Spannungs- und Verformungsverhalten des Bodens bei mechanischer Belastung. Technische Universität Braunschweig, Arbeitsgruppe 
Bodenzoologie: Auswirkung mechanischer Belastung auf die Bodenfauna. Die vorliegende Arbeit stellt den Beitrag der Arbeitsgruppe Göttingen dar.

Die über Jahre unterschiedlich bearbeiteten Böden der Standorte wurden mechanisch mit Fahrzeugen belastet, die sich durch zwei Achsen und vier gleich große Räder auszeichneten. Die Gesamtlast konnte durch Zuladung von Gewichten gleichmäßig auf Achsen und Räder verteilt werden. Dies sollte die Interpretation der Belastungswirkung vereinfachen. Die Achslast der Fahrzeuge wurde auf $5 \mathrm{t}$ und 10t festgelegt, um praxisübliche, schwere Achslasten zu simulieren. Als Beispiel für praxisübliche Achslasten können ein schwerer Schlepper mit 4t Achslast, ein gefülltes, großvolumiges Güllefaß mit 8t Achslast und ein selbstfahrender Köpfbunkerroder mit Achslasten bis zu 15t (ZIEGLER 1995) gelten.

Durchgeführt wurde die Befahrung auf zwei Standorten in Niedersachsen mit Radladern der Firma Orenstein \& Koppel, Dortmund. Auf dem dritten Standort in Thüringen kamen ein Kranwagen und ein Radlader der Firma Ahlmann zum Einsatz. Die technischen Daten und die Gewichte der Fahrzeuge sowie deren Kontaktflächendrücke finden sich in Tab. 38, 39 im Anhang.

Die mechanische Belastung der Böden wurde flächendeckend aufgebracht. Dies wurde erreicht, indem die Spuren der Fahrzeuge ohne Überlappung oder Zwischenraum aneinander gesetzt wurden. Die Überrollgeschwindigkeit bei der Belastung entsprach in etwa $4 \mathrm{~km} / \mathrm{h}$. Sinn der flächendeckenden Belastung war es, Randeffekte durch nicht belastete Bereiche zu vermeiden. Auch erschien die flächenhafte Belastung gerechtfertigt, da praxisübliche pflanzenbauliche Maßnahmen wie die Ernte von Mais oder Hackfrüchten ebenfalls zu einer stark flächigen Überrollung führen (Brandhuber 1997).

In der pflanzenbaulichen Praxis kommt es oft vor, daß schwere Maschinen wie Güllefässer, selbstfahrende Köpfrodebunker, schwere Kipper oder Silomaisernter bei feuchtem Boden im Herbst zum Einsatz kommen. Dies kann besonders drastische Folgen haben, da Böden im feuchten Zustand instabil und damit besonders anfällig für Gefügeveränderungen sind. In der vorliegenden Arbeit sollte die Auswirkung der mechanischen Belastung auf den Boden unter solch „ungünstigen“ Bedingungen geprüft werden. Um sicherzustellen, daß die Böden der Standorte bei der mechanischen Belastung zu Versuchsbeginn einen nicht 
zu geringen Wassergehalt aufwiesen, wurde der Boden auf allen drei Standorten im Frühjahr bei Wassergehalten nahe der Feldkapazität belastet.

Nach der mechanischen Bodenbelastung wurde die erste Versuchsfrucht ausgesät. Die Aussaat erfolgte nach der Bodenbelastung, da nicht die kombinierte Wirkung aus mechanischer Verletzung des Saatgutes oder der aufgelaufenen Pflanzen und der geänderten Bodeneigenschaften geprüft werden sollte.

Der Boden unter Pflug wurde nach der Bodenbelastung und vor der Einsaat nicht gepflügt, um im ersten Versuchsjahr die direkte Wirkung der Bodenbelastung ohne mechanische Auflockerung zu erfassen und die unterschiedliche Empfindlichkeit der Bearbeitungssysteme gegen mechanische Belastung zeigen zu können. Nach der Bodenbelastung und der Aussaat im ersten Versuchsjahr verliefen die pflanzenbaulichen Maßnahmen betriebsüblich. Auf LBW wurde zu jeder Frucht gepflügt. 
3 Material und Methoden

3.1 Versuchsanlage und Standortbeschreibung

3.1.1 Einrichtung der mechanischen Bodenbelastung

Auf allen drei Standorten wurden zusätzlich zur betriebsüblichen mechanischen Bodenbelastung im Frühjahr 1995 auf den im Herbst gepflügten oder flach bearbeiteten Boden folgende Radlasten aufgebracht:

1. Belastungsstufe: $0 t ;$ keine zusätzliche mechanische Bodenbelastung

2. Belastungsstufe: 2,5t; einmaliges Überfahren mit zwei Achsen

3. Belastungsstufe: $5 t$; einmaliges Überfahren mit zwei Achsen

4. Belastungsstufe: $5 t$; dreimaliges Überfahren mit zwei Achsen

Tab. 1 faßt Abkürzungen und Bezeichnungen der Belastungsstufen zusammen.

Tab. 1: Kennzeichnung der Belastungsstufen.

\begin{tabular}{l|cc} 
& Überrollhäufigkeit x Radlast & Belastungsintensität \\
\hline 1. Belastungsstufe & $0 \mathrm{t}$ & keine \\
2. Belastungsstufe & $2 \times 2,5 \mathrm{t}$ & gering \\
3. Belastungsstufe & $2 \times 5 \mathrm{t}$ & mittel \\
4. Belastungsstufe & $6 \times 5 \mathrm{t}$ & hoch
\end{tabular}

Auf allen drei Standorten wurde die mechanische Bodenbelastung bei Frühjahrssättigung der Böden durchgeführt. Tab. 2 und 3 geben Auskunft über den Zeitpunkt der Versuchsanlage sowie über die Wassergehalte und die Feldkapazität der Böden zu Versuchsbeginn. Die Versuchseinrichtung begann in Thüringen und endete in Relliehausen am Sollingrand. 
Tab. 2: Termine der Versuchsanlage an den Standorten in Niedersachsen (Reinshof, Relliehausen) und in Thüringen (Großobringen).

\begin{tabular}{c|ccc} 
& \multicolumn{3}{|c}{ Standort } \\
\hline & Reinshof & Relliehausen & Großobringen \\
Termin & $12 . / 13.04 .1995$ & $25 . / 26.04 .1995$ & $23 . / 24.03 .1995$
\end{tabular}

Tab. 3: Bodenwassergehalt (BWG) und Feldkapazität (FK) der Böden bei der mechanischen Belastung zu Versuchsbeginn auf den Standorten Reinshof und Relliehausen.

\begin{tabular}{l|cc} 
& \multicolumn{2}{|c}{ Standort } \\
\hline BWG [Vol.\%] in der & Reinshof & Relliehausen \\
\cline { 2 - 3 } Bodenschicht 0-30 cm & 32,1 & 35,1 \\
BWG [Vol.\%] in der & & 34,5 \\
Bodenschicht 30-100 cm & 33,9 \\
FK [Vol.\%] in der & & \\
Bodenschicht 0-30 cm & 31,5 & 34,2 \\
FK [Vol.\%] in der Boden- & & 35,8 \\
schicht 30-100 cm & 32,5 &
\end{tabular}


Tab. 4: Bodenwassergehalt (BWG) und Feldkapazität (FK) der Böden bei der mechanischen Belastung zu Versuchsbeginn auf dem Standort Großobringen. Die Daten sind zusammengestellt nach Angaben der Thüringer Landesanstalt für Landwirtschaft, Jena.

\begin{tabular}{l|c} 
& Standort \\
\hline BWG [Vol.\%] in der & Großobringen \\
Bodenschicht 0-30 cm & 34,0 \\
BWG [Vol.\%] in der & \\
Bodenschicht 30-40 cm & 34,2 \\
FK [Vol.\%] in der & \\
Bodenschicht 0-30 cm & 33,5 \\
FK [Vol.\%] in der Boden- & \\
schicht 30-40 cm & 33,5
\end{tabular}

\subsubsection{Pflanzenbauliche Maßnahmen}

Die mechanische Bodenbelastung erfolgte auf den Standorten von Ende März bis Ende April (Tab. 2). Die erste der Belastung folgende Feldfrucht mußte spätsaatverträglich sein. Aus diesem Grund fiel die Wahl auf Sommergerste. Als ein weiterer Vorteil der Sommergerste wurde ihre Empfindlichkeit gegenüber ungünstigen Bodenverhältnissen wie Bodenverdichtungen angesehen. Der Sommergerste folgte im Herbst 1995 auf allen Standorten Winterweizen. Die Feldfrucht des dritten Versuchsjahres war auf allen Standorten Wintergerste. Winterweizen und Wintergerste wurden auf Grund ihrer allgemeinen Verbreitung und ihrer Bedeutung in standortüblichen Fruchtfolgen in der praktischen Landwirtschaft angebaut. Auf den Anbau von Zwischenfrüchten oder Untersaaten wurde grundsätzlich verzichtet. Ein Verzeichnis der betriebsüblichen pflanzenbaulichen Maßnahmen auf den drei Standorten über die Versuchsjahre findet sich in Tab. 57, 58 und 59 im Anhang. 


\subsubsection{Standort Reinshof}

\subsubsection{Versuchsfläche}

Die Versuchsfläche ist ein Teil des Versuchsgutes Reinshof der Universität Göttingen und liegt im Leinetal ca. 8 km südlich von Göttingen, 165 m ü. NN.

Der Boden des Versuchsfeldes wird als kolluvial überprägte Parabraunerde beschrieben. Das Ausgangsmaterial für die Bodenbildung war Löß. In Tab. 5 ist die Horizontfolge und die Korngrößenzusammensetzung dargestellt.

Tab. 5: Horizontfolge und Korngrößenzusammensetzung des Bodens auf der Versuchsfläche Reinshof. Dargestellt ist der Anteil der Fraktionen [\%].

\begin{tabular}{|c|c|c|c|c|c|c|c|c|}
\hline \multirow[b]{3}{*}{ Horizont } & \multicolumn{8}{|c|}{ Korndurchmesser $[\mu \mathrm{m}]$} \\
\hline & & Ton & & Schluft & & & Sand & \\
\hline & Tiefe $[\mathrm{cm}$ & $<2$ & $2-6$ & $6-20$ & $20-60$ & $60-200$ & $200-600$ & $600-2000$ \\
\hline Ap & $0-30$ & 15,1 & 2,8 & 16,6 & 53,3 & 6,6 & 5,3 & 0,3 \\
\hline$M$ & $30-50$ & 14,9 & 4,3 & 17,9 & 52,2 & 6,0 & 4,6 & 0,1 \\
\hline $\mathrm{Bt}$ & $>50$ & 21,6 & 1,7 & 16,7 & 49,8 & 5,7 & 4,5 & 0,1 \\
\hline
\end{tabular}

Auf dem Standort Reinshof wurde die mechanische Bodenbelastung in einen seit 1970 bestehenden Versuch zum Vergleich der Bearbeitungsysteme LBW und FMW integriert. Abb. 1 zeigt den Parzellenplan des Versuches. 


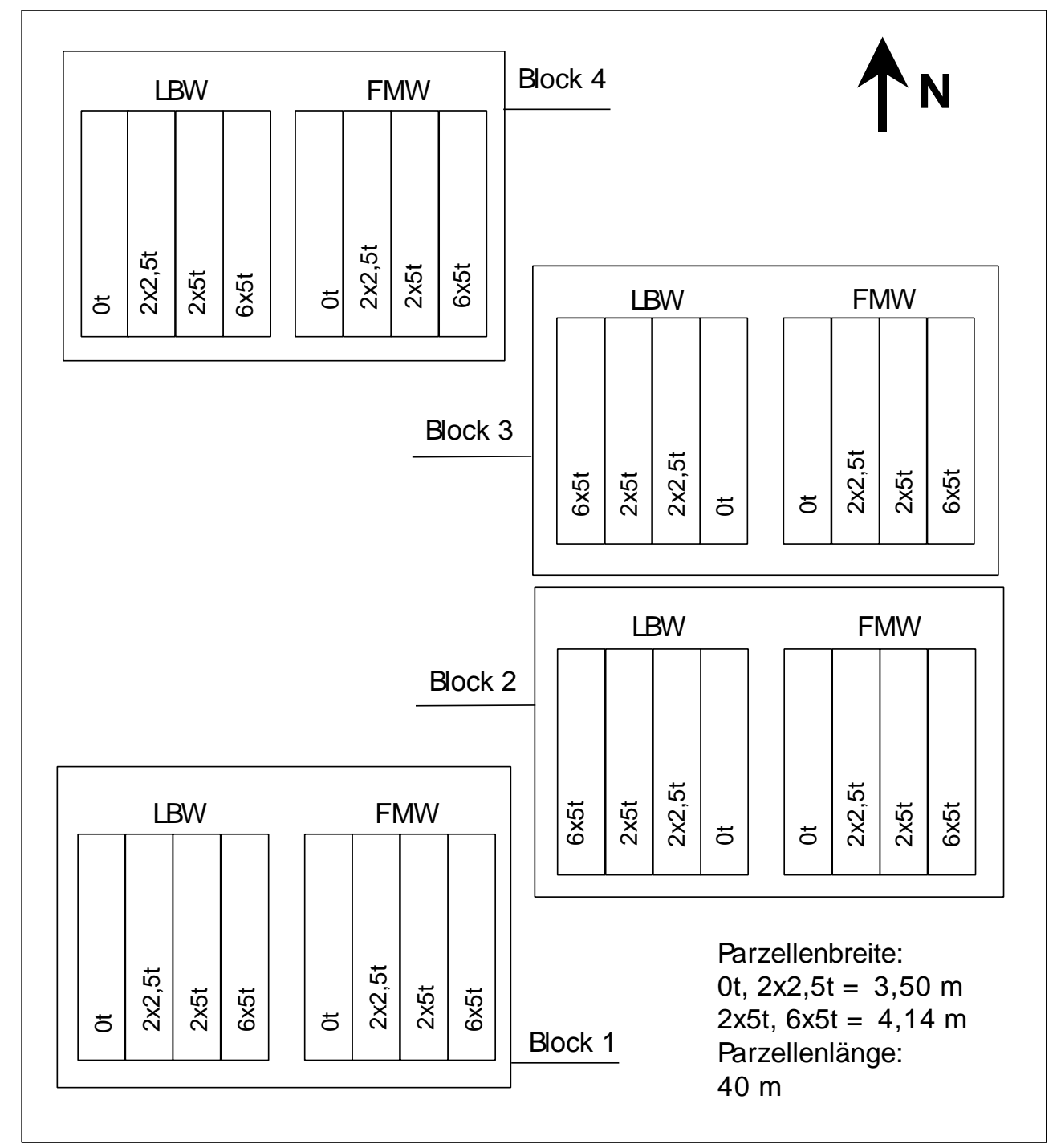

Abb. 1: Versuchsplan Standort Reinshof. Die Darstellung ist nicht maßstabgetreu.

Es standen zu Versuchsbeginn Flächen mit langjährig wendender und nichtwendender Bodenbearbeitung zur Verfügung. Der Feldversuch wurde als zweifaktorielle Blockanlage mit Untereinheiten angelegt. Verglichen werden sollten die beiden Faktoren Bodenbearbeitungssystem und mechanische Bodenbelastung. Unter dem Faktor Bodenbearbeitungssystem standen die beiden Stufen LBW und FMW zur Verfügung. Beim Faktor mechanische Bodenbelastung wurden die Belastungsstufen 0t, 2x2,5t, 2x5t und 6x5t verglichen (Tab. 1). Insgesamt wurden also acht Varianten geprüft. Der Versuch setzte sich aus vier vollständigen Blöcken zusammen. Aus versuchstechnischen Gründen wurden 
die Belastungsstufen innerhalb der Blöcke systematisch in der Reihenfolge 0t, $2 \times 2,5 t, 2 \times 5 t$ und $6 \times 5 t$ angelegt.

\subsubsection{Klima}

Die Jahresdurchschnittstemperatur entsprach in den Jahren 1995 und 1997 in etwa dem langjährigen Mittel, war jedoch 1996 mit 7,1 ${ }^{\circ} \mathrm{C}$ deutlich geringer (Abb. 2).

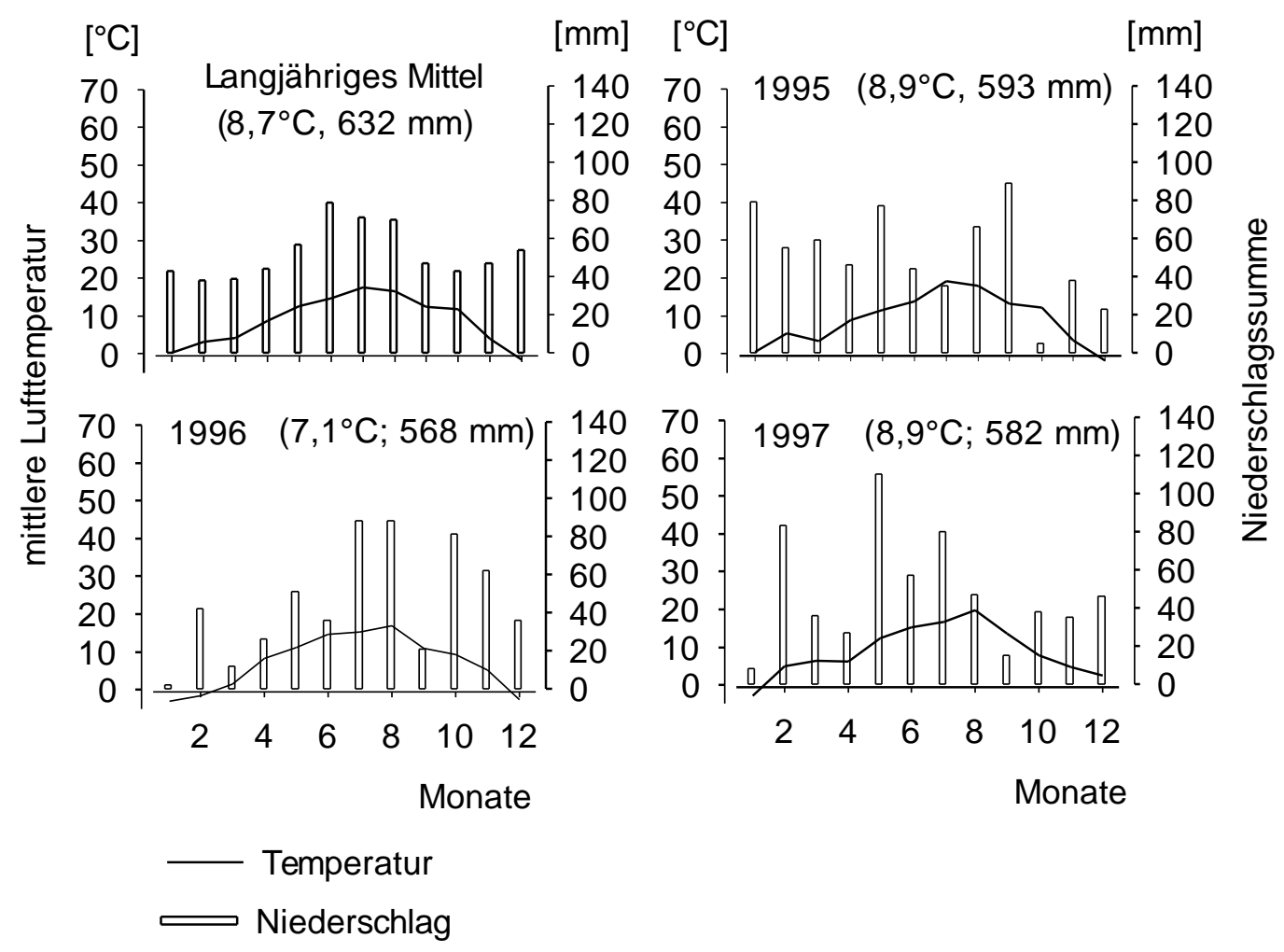

Abb. 2: Niederschläge (Monatssummen) und Lufttemperatur (Monatsmittel) als langjähriges Mittel (1951-1980) und in den Jahren 1995, 1996 und 1997 auf dem Standort Reinshof. Die Zahlen in Klammern kennzeichnen die Jahresduchschnittstemperatur und die Jahresniederschlagsmenge. Die Daten wurden zusammengestellt nach Angaben des Deutschen Wetterdienstes, Wetterstation Göttingen.

Die Jahresniederschlagsmenge lag in allen drei Versuchsjahren unter dem langjährigen Mittel. Die Verteilung der Niederschläge über das Jahr zeigte in allen Versuchsjahren eine deutliche Abweichung vom langjährigen Mittel: Im ersten Versuchsjahr schwankte die Niederschlagsmenge pro Monat stark. Die beiden Monate Juni und Juli fielen im Vergleich mit dem langjährigen Mittel durch besonders geringe Monatssummen auf. Das zweite Versuchsjahr zeich- 
nete sich durch ein niederschlagsarmes Frühjahr und eine regenreiche zweite Jahreshälfte aus. Im Frühjahr des dritten Versuchsjahres lagen die Monatssummen der Monate Februar und Mai erheblich über dem langjährigen Mittel. Mit Ausnahme des trockenen Septembers erreichten die Niederschläge in der zweiten Jahreshälfte annähernd das Niveau des langjährigen Mittels.

\subsubsection{Standort Relliehausen}

\subsubsection{Versuchsfläche}

Die Versuchsfläche liegt am Nord-Ost-Rand des Solling ca. $190 \mathrm{~m}$ ü. NN und ist ein Teil des Versuchsgutes Relliehausen der Universität Göttingen. Der Boden der Versuchsfläche entstand auf einer bis zu $3 \mathrm{~m}$ mächtigen Lößdecke über Hangschutt des mittleren Buntsandsteins. Nach kompletter Entkalkung des primär kalkhaltigen Substrates entwickelte sich eine Pseudogley-Parabraunerde. Tab. 6 zeigt die Horizontfolge und die Korngrößenzusammensetzung des Bodens.

Tab. 6: Horizontfolge und Korngrößenzusammensetzung des Bodens auf der Versuchsfläche Relliehausen. Dargestellt ist der Anteil der Fraktionen [\%].

\begin{tabular}{|c|c|c|c|c|c|c|c|c|}
\hline \multirow[b]{3}{*}{ Horizont } & \multicolumn{8}{|c|}{ Korndurchmesser $[\mu \mathrm{m}]$} \\
\hline & \multirow[b]{2}{*}{ Tiefe $[\mathrm{cm}]$} & \multirow{2}{*}{$\frac{\text { Ton }}{<2}$} & \multicolumn{3}{|c|}{ Schluff } & \multicolumn{3}{|c|}{ Sand } \\
\hline & & & $2-6$ & $6-20$ & $20-60$ & $60-200$ & $200-600$ & $600-2000$ \\
\hline Ap1 & $0-16$ & 12,9 & 3,1 & 20,4 & 37,4 & 24,4 & 1,2 & 0,6 \\
\hline Ap2 & $16-30$ & 11,9 & 4,0 & 20,5 & 36,1 & 25,9 & 1,0 & 0,3 \\
\hline Ap3 & $30-38$ & 11,7 & 4,2 & 21,3 & 36,8 & 24,5 & 1,0 & 0,3 \\
\hline Al & $38-50$ & 12,6 & 3,4 & 21,9 & 39,4 & 21,1 & 1,1 & 0,2 \\
\hline Btg & $>50$ & 18,4 & 2,9 & 22,2 & 32,6 & 22,8 & 1,6 & 0,1 \\
\hline
\end{tabular}

Die auf dem Standort Relliehausen zur Verfügung stehende Fläche wurden seit 1990 mit den Bearbeitungssystemen LBW und FMW bewirtschaftet. Der Belastungsversuch wurde auf dem bestehenden Versuch zu Bodenbearbeitung eingerichtet. Aus Platzgründen wurden nur zwei Wiederholungen angelegt. Die östliche Wiederholung stand ausschließlich der AG Braunschweig zur Verfügung (Abb. 3). 
Verglichen wurden die acht Varianten der beiden Faktoren Bearbeitungssystem und mechanische Bodenbelastung mit den Stufen LBW, FMW sowie 0t, 2x2,5t, 2x5t und 6x5t. Abb. 3 verdeutlicht die Lage und Ausrichtung der Parzellen.

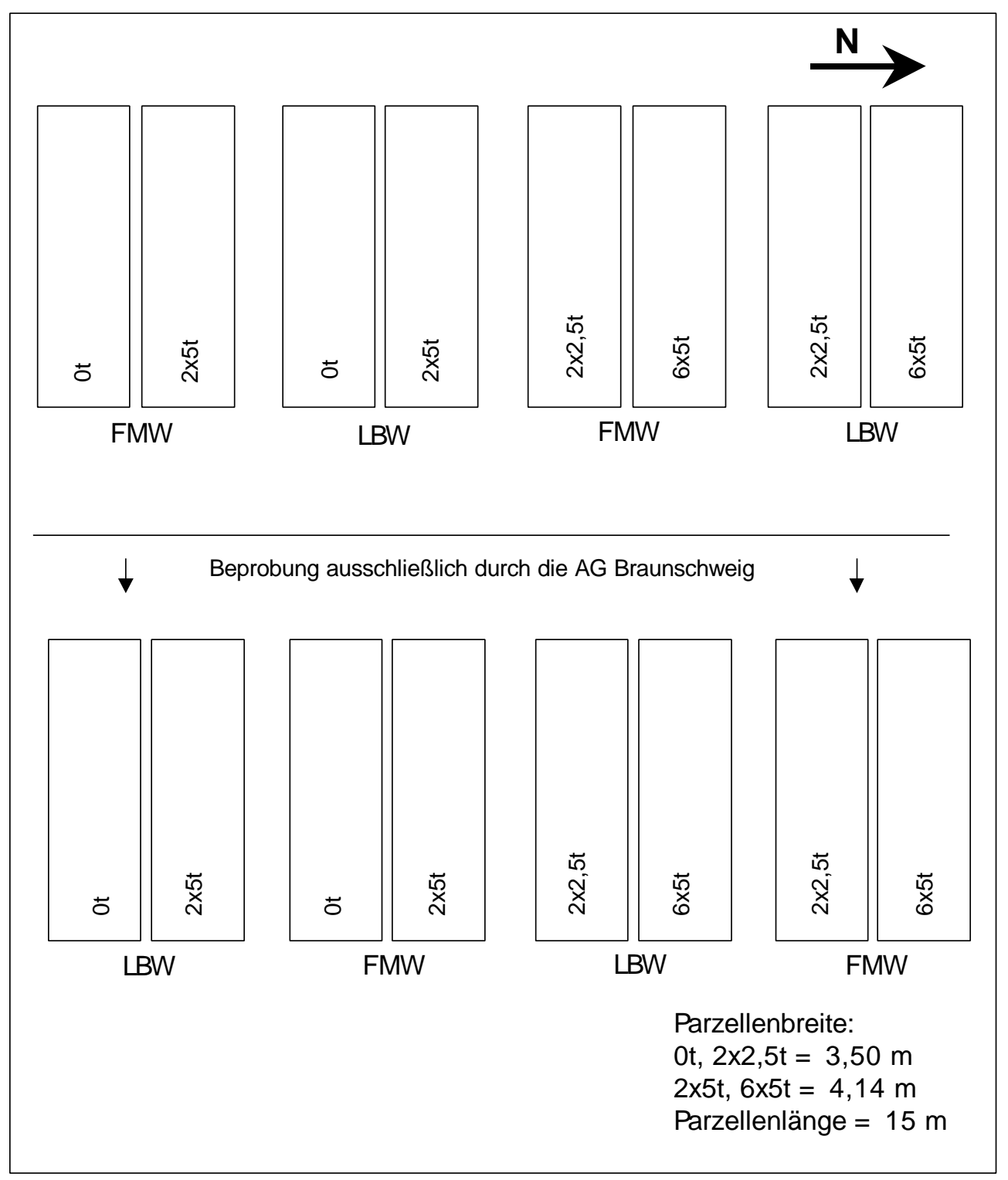

Abb. 3: Versuchsplan Standort Relliehausen. Die Darstellung ist nicht maßstabgetreu. 


\subsubsection{Klima}

Abb. 4 zeigt die Niederschlagsentwicklung der Versuchsjahre im Vergleich zum langjährigen Mittel und die mittlere Lufttemperatur auf dem Standort Relliehausen (190 m. ü. NN). Da für den Standort Relliehausen keine Temperaturmessungen zur Verfügung standen, wurden Messungen aus Silberborn (440 m ü.NN) übertragen. Der Höhenunterschied wurde mit $0,5^{\circ} \mathrm{C}$ Temperaturabfall bei 100 m Höhenzunahme berücksichtigt (WEISCHET 1995). Die Entfernung zwischen Silberborn und Relliehausen beträgt etwa 14 km, der Höhenunterschied $250 \mathrm{~m}$. Um die Temperaturen von Silberborn auf Relliehausen zu übertragen wurden $1,25^{\circ} \mathrm{C}$ addiert.

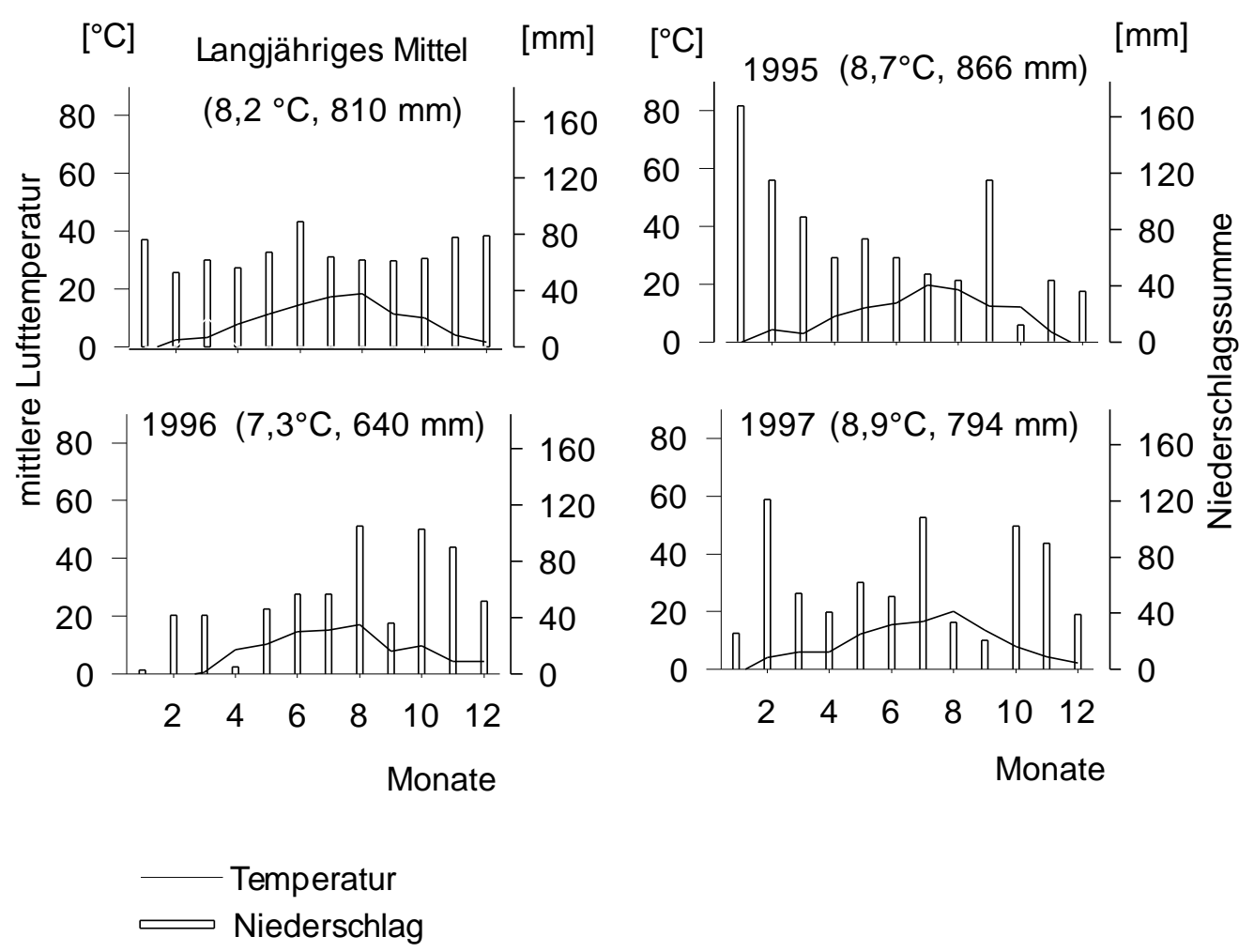

Abb. 4: Niederschläge (Monatssummen) als langjähriges Mittel (1969-1997) und in den Versuchsjahren 1995, 1996 und 1997 sowie die Lufttemperatur (Monatsmittel, 1961-1990 und 1995, 1996 und 1997). Die Daten wurden zusammengestellt nach Messungen auf dem Versuchsgut Relliehausen, in $2 \mathrm{~km}$ Entfernung vom Versuchsfeld (Niederschläge) und nach Angaben des Deutschen Wetterdienstes, Wetterstation Silberborn (Lufttemperatur). 
Das erste Versuchsjahr zeichnete sich durch ein feuchtes Frühjahr und einen trockenen Herbst aus. Auffällig ist der September mit ungewöhnlich hoher Niederschlagsmenge. Die Jahresniederschlagsmenge lag $56 \mathrm{~mm}$ über dem langjährigen Mittel. Im Jahr 1996 lag die Niederschlagsmenge in der ersten Jahreshälfte deutlich unter dem langjährigen Mittel. Das Niveau der Niederschlagsmenge der zweiten Jahreshälfte entsprach in etwa dem des langjährigen Mittels. Insgesamt war das zweite Versuchsjahr deutlich trockener.

Im dritten Versuchsjahr waren die Niederschläge annähernd gleichmäßig über das Jahr verteilt. Der Jahresniederschlag entsprach in etwa dem des langjährigen Mittels. In den Jahren 1995 und 1997 lag die Jahresdurchschnittstemperatur geringfügig über dem langjährigen Mittel. Im Jahr 1996 lag die Durchschnittstemperatur um $1,0^{\circ} \mathrm{C}$ unter dem langjährigen Mittel.

\subsubsection{Standort Großobringen}

\subsubsection{Versuchsfläche}

Die Versuchsfläche bei Großobringen wurde von der Thüriger Landesanstalt für Landwirtschaft zur Verfügung gestellt und betreut. Die Fläche liegt ca. 7 km nördlich von Weimar am Randgebiet des Thüringer Beckens , 240 m ü. NN.

Der Boden der Versuchsfläche wird als flachgründige, lößbeeinflußte Schwarzerde über Keuperton beschrieben. Tab. 7 zeigt die Horizontabfolge und die Korngrößenzusammensetzung des Bodens.

Tab. 7: Horizontabfolge und Korngrößenzusammensetzung des Bodens auf der Versuchsfläche Großobringen. Dargestellt ist der Anteil der Fraktionen [\%]. Die Horizontabfolge und die Daten sind zusammengestellt nach den Angaben der Thüringer Landesanstalt für Landwirtschaft, Jena.

\begin{tabular}{|c|c|c|c|c|c|c|c|c|}
\hline \multirow[b]{3}{*}{ Horizont } & \multicolumn{8}{|c|}{ Korndurchmesser $[\mu \mathrm{m}]$} \\
\hline & \multirow[b]{2}{*}{ Tiefe $[\mathrm{cm}]$} & \multirow{2}{*}{$\frac{\text { Ton }}{<2}$} & \multicolumn{3}{|c|}{ Schluff } & \multicolumn{3}{|c|}{ Sand } \\
\hline & & & $2-6$ & $6-20$ & $20-60$ & $60-200$ & $200-600$ & $600-2000$ \\
\hline Ap1 & $0-5$ & 31,4 & 6,6 & 21,2 & 27,8 & 10,8 & 1,8 & 0,5 \\
\hline Ap2 & $5-20$ & 31,8 & 6,4 & 23,2 & 25,4 & 11,0 & 1,8 & 0,3 \\
\hline Ap3 & $20-30$ & 32,0 & 6,5 & 22,5 & 27,0 & 9,8 & 1,9 & 0,4 \\
\hline Ah & $30-55$ & 30,9 & 4,5 & 23,3 & 26,4 & 13,2 & 1,4 & 0,2 \\
\hline$\| \mathrm{Cc}$ & $55-70$ & 22,0 & 5,8 & 24,0 & 30,1 & 15,5 & 1,9 & 0,6 \\
\hline
\end{tabular}


Im Vergleich der Standorte fällt in Großobringen der besonders hohe Tongehalt des Bodens auf. Aufgrund des hohen Tonanteils wurde der Boden in Großobringen - vor allem bei einem hohen Bodenwassergehalt - als besonders belastungsempfindlich eingeschätzt.

$\mathrm{Zu}$ Versuchsbeginn standen aus einem Vorversuch (seit 1990) tief $(25 \mathrm{~cm})$ und flach $(12 \mathrm{~cm}$ ) bearbeitete Teilflächen zur Verfügung. Die lockernde und wendende Bodenbearbeitung wurde mit der Spatenmaschine durchgeführt.

Der Versuch wurde als Langparzellenversuch ohne Wiederholung angelegt. Unter den beiden Faktoren Bearbeitungssystem und mechanische Bodenbelastung wurden die Stufen LBW tief, LBW flach sowie 0t, 2x2,5t, 2x5t und 6x5t variiert. Insgesamt wurden acht Varianten verglichen. Abb. 5 zeigt die Lage und Ausrichtung der Parzellen.

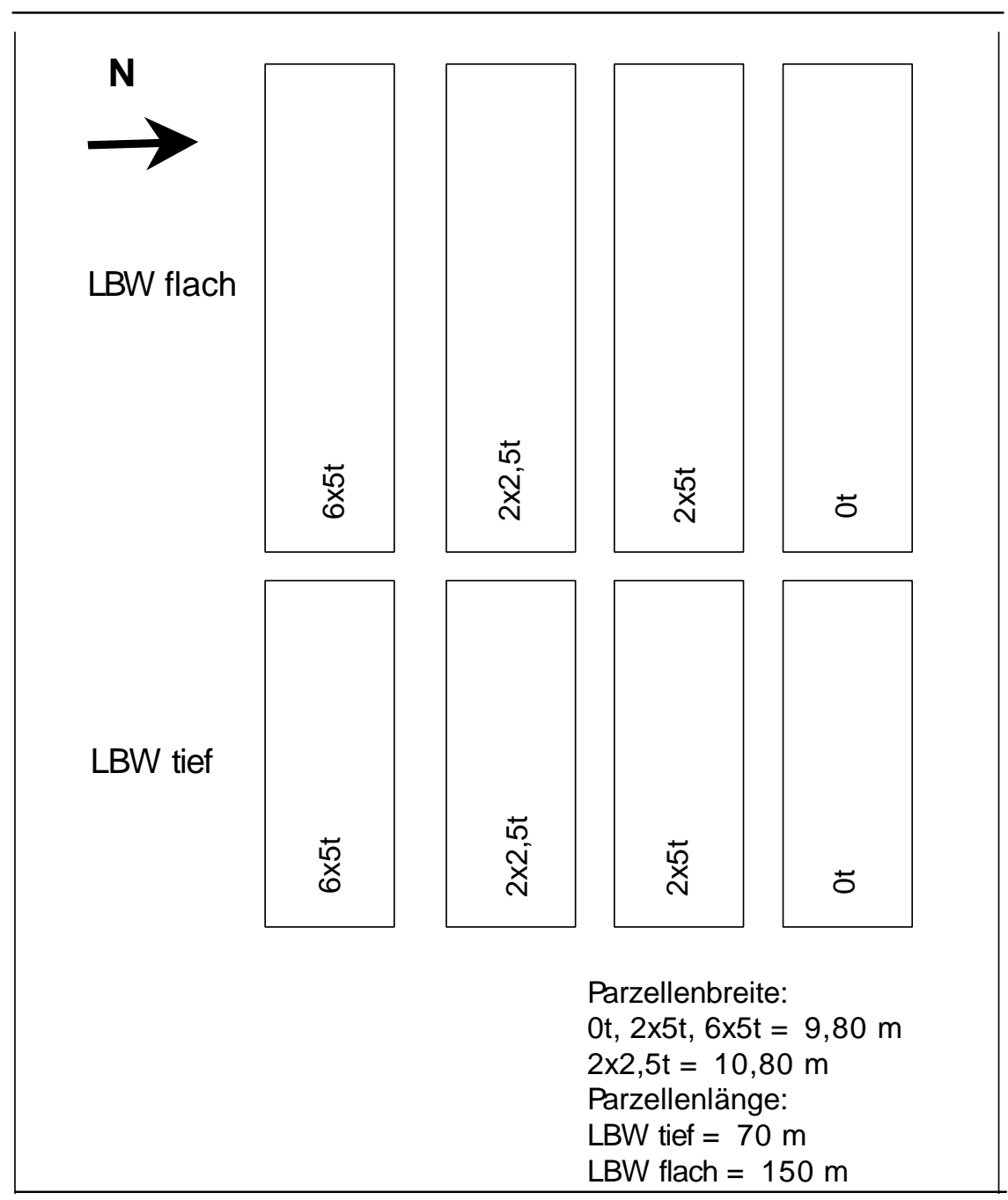

Abb. 5: Versuchsplan Standort Großobringen. Die Darstellung ist nicht maßstabgetreu. 


\subsubsection{Klima}

Das erste Versuchsjahr zeichnete sich durch überdurchschnittlich hohe Niederschläge in den Monaten Mai, Juni, Juli und September aus (Abb. 6). Das letzte Drittel des Jahres war dagegen deutlich trockener als das langjährige Mittel.

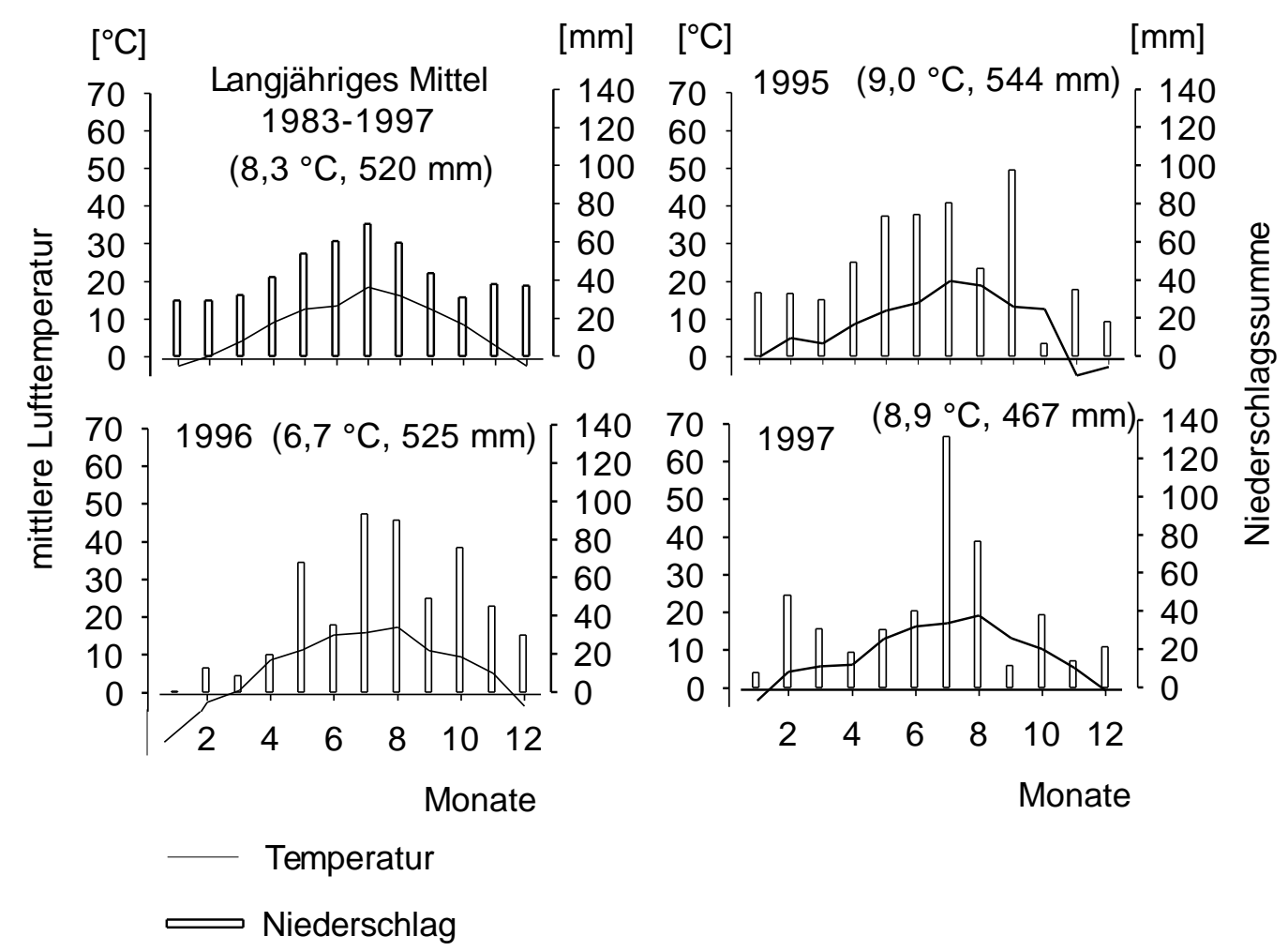

Abb. 6: Niederschläge (Monatssummen) und Lufttemperatur (Monatsmittel) als langjähriges Mittel (1983-1997) und in den Jahren 1995, 1996 und 1997 auf dem Standort Großobringen. Die Zahlen in Klammern kennzeichnen die Jahresdurschnittstemperatur und die Jahresniederschlagsmenge. Die Daten wurden zusammengestellt nach Angaben der Thüringer Landesanstalt für Landwirtschaft, Jena.

Das zweite Versuchsjahr zeichnete sich durch einen überdurchschnittlich kalten Jahresbeginn aus. Insgesamt lag die Jahresdurchschnittstemperatur deutlich unter dem langjährigen Mittel. Mit 525 mm entsprach die Jahresniederschlagssumme in etwa dem des langlährigen Mittels. Die Niederschlagsverteilung über das Jahr wies jedoch Besonderheiten auf: Im ersten Jahresdrittel fiel im Vergleich zum langlährigen Mittel deutlich weniger Niederschlag. Die Monate Juli, August und Oktober zeichneten sich durch hohe Niederschläge aus. Das dritte Versuchsjahr zeichnete sich durch eine außergewöhnlich niedrige Nieder- 
schlagssumme aus. Im Frühjahr und im Herbst lag die Niederschlagsmenge beträchtlich unter dem langjährigen Mittel. Besonders auffällig ist der Monat Juli: In diesem Monat fielen mit $131 \mathrm{~mm}$ ca. $30 \%$ des gesamten Jahresniederschlags. Die Jahresdurchschnittstemperatur wich 1997 mit 8,9 ${ }^{\circ} \mathrm{C}$ nur gering vom langjährigen Mittel ab.

Im Vergleich der Standorte wurde in Großobringen in den drei Versuchsjahren mit durchschnittlich $512 \mathrm{~mm}$ die geringste Jahresniederschlagssumme gemessen. Der feuchteste Standort war Relliehausen mit durchschnittlich $766 \mathrm{~mm}$ Niederschlag in den Versuchsjahren. Göttingen lag mit 581 mm mittlerer Jahresniederschlagssumme zwischen den beiden anderen Standorten.

\subsection{Bodenphysikalische Untersuchungen}

\subsubsection{Bodenwassergehalt}

Der gravimetrische Bodenwassergehalt [Gew.\%] in Bohrstockproben wurde durch Wiegen vor und nach der Trocknung bei $105^{\circ} \mathrm{C}$ bis zur Gewichtskonstanz ermittelt. Alle Angaben zum gravimetrischen Bodenwassergehalt beruhen auf der Trockensubstanz (TS) als 100\% Basis. Zur Berechnung des volumetrischen Wassergehalts [Vol.\%] einer Probe wurde der gravimetrische Bodenwassergehalt mit der Lagerungsdichte der entsprechenden Bodenschicht multipliziert. Die Werte der Lagerungsdichte stellte die AG Jena zur Verfügung. Entsprach der Entnahmehorizont zur Bestimmung der Lagerungsdichte nicht exakt der Bodenschicht zur Bestimmung des Wassergehaltes, wurde die Lagerungsdichte aus den Werten der angrenzenden Bodenschichten interpoliert.

Die Differenz des Bodenwassergehaltes [Vol.\%] vom 04.06.1997 bis zum 19.06.1997 gibt die Bodenwasserausschöpfung durch die Kulturpflanzen an. In dieser Zeitspanne fielen keine Niederschläge. Die Angabe Bodenwassergehalt [Vol.\%] entspricht der Angabe Bodenwassergehalt [mm/dm Bodenschicht]. Zur Diskussion der Bodenwasserausschöpfung wurden zwei Annahmen gemacht: Eine Wassernachlieferung aus angrenzenden Bodenschichten fand nicht statt. Die Wasserverluste durch Evaporation können unberücksichtigt bleiben. Tab. 8 und 9 zeigen eine Zusammenfassung der Beprobungsdaten zum Bodenwassergehalt. 
Tab. 8: Beprobungsplan Bodenwassergehalt auf den Standorten Reinshof (RH), Relliehausen (RL) und Großobringen (GO). Die Beprobung wurde während der Vegetation durchgeführt. Die Abkürzungen bedeuten: $\mathbf{B o}=$ untersuchtes Bearbeitungssystem; $\mathbf{B s}=$ untersuchte Belastungsstufen; $\mathbf{B I}=$ untersuchte Blöcke auf dem Standort RH; $\mathbf{n}=$ Anzahl der Meßwiederholungen pro Parzelle und Beprobungstermin; $\mathbf{T}$ = Tiefe der untersuchten Bodenschicht in [cm].

\begin{tabular}{|c|c|c|c|c|c|}
\hline Standor & Bo & Bs & BI & $n$ & $\mathbf{T}$ \\
\hline \multirow{2}{*}{$\mathbf{R H}$} & & $1-4$ & Block 2 & 3 & $0-100$ \\
\hline & FMW & & & & $10 \mathrm{~cm}$-Abschnitte \\
\hline \multirow{2}{*}{$\mathbf{R L}$} & LBW und & $1-4$ & - & 3 & $0-100$ \\
\hline & FMW & & & & $10 \mathrm{~cm}$-Abschnitte \\
\hline \multirow{2}{*}{ GO } & LBW tief und & $1-4$ & - & 3 & $0-40$ \\
\hline & LBW flach & & & & $10 \mathrm{~cm}$-Abschnitte \\
\hline
\end{tabular}

Das Muster und die Abkürzungen dieser Tabelle werden beibehalten, um die Probenahme der folgenden Methoden zu beschreiben.

Tab. 9: Beprobungszeitpunkte (Bt) zum Bodenwassergehalt auf den Standorten Reinshof (RH), Relliehausen (RL) und Großobringen (GO).

\begin{tabular}{|c|c|c|c|}
\hline & & $\mathrm{Bt}$ & \\
\hline \multirow[t]{2}{*}{ Standort } & \multicolumn{3}{|c|}{ Versuchsjahr } \\
\hline & 1995 & 1996 & 1997 \\
\hline $\mathbf{R H}$ & $\begin{array}{l}04.04 \\
04.05 . \\
13.06 . \\
27.06 . \\
20.07 . \\
01.08 .\end{array}$ & $\begin{array}{l}16.04 . \\
22.04 . \\
08.05 \\
22.05 . \\
05.06 \\
16.06 \\
03.07 \\
15.07 \\
01.08 \\
15.08\end{array}$ & $\begin{array}{l}19.03 . \\
16.04 \text {. } \\
07.05 \\
20.05 . \\
03.06 \text {. } \\
18.06 \\
02.07 \\
16.07 .\end{array}$ \\
\hline $\mathbf{R L}$ & $\begin{array}{c}13.05 \\
21.06 \\
5.07 \\
1.08\end{array}$ & $\begin{array}{l}29.04 \\
29.05 \\
26.06 \\
25.07\end{array}$ & $\begin{array}{l}13.03 . \\
10.06\end{array}$ \\
\hline GO & wöchentlich & wöchentlich & wöchentlich \\
\hline
\end{tabular}




\subsubsection{Eindringwiderstand}

Die Messung des Eindringwiderstandes (EDW) wurde 1995 in RH mit einem selbstregistrierenden Penetrographen der Firma Eijkelkamp (Typ 06.02, mechanische Aufzeichnung) durchgeführt. In diesem Versuchsjahr konnten auf den Standorten RL und GO aus technischen Gründen keine Messungen durchgeführt werden. Zur Messung 1996 und 1997 wurde auf allen Standorten ein selbstregistrierender Penetrograph der Firma Eijkelkamp (Typ 06.05, elektronische Aufzeichnung) eingesetzt. Bei einem Öffnungswinkel von $60 \mathrm{Grad}$ betrug die Fläche der Kegelspitze $1 \mathrm{~cm}^{2}$. Die Eindringgeschwindigkeit wurde auf näherungsweise $8 \mathrm{~cm} / \mathrm{s}$ festgelegt. Die maximale Untersuchungstiefe betrug $70 \mathrm{~cm}$. Die zu den EDW im Anhang dargestellten Lagerungsdichten sind von der AG Jena ermittelt und zur Verfügung gestellt worden. In Tab. 10 ist der Beprobungsplan zur Bestimmung des EDW dargestellt.

Tab. 10: Beprobungsplan Eindringwiderstand; Zur Erläuterung der Abkürzungen vergl. Tab. 8.

\begin{tabular}{|c|c|c|c|c|c|c|}
\hline Standor & $B t$ & Bo & Bs & BI & $\mathrm{n}$ & $\mathbf{T}$ \\
\hline $\mathbf{R H}$ & 04.04.1995 & $\begin{array}{l}\text { LBW und } \\
\text { FMW }\end{array}$ & $1-4$ & Block 2 & 15 & $0-70 \mathrm{~cm}$ \\
\hline $\mathbf{R H}$ & $\begin{array}{l}16.04 .1996 \\
19.03 .1997\end{array}$ & $\begin{array}{l}\text { LBW und } \\
\text { FMW }\end{array}$ & $1-4$ & Block 1-4 & 10 & $0-70 \mathrm{~cm}$ \\
\hline RL & \begin{tabular}{|l|}
29.04 .1996 \\
13.03 .1997
\end{tabular} & $\begin{array}{l}\text { LBW und } \\
\text { FMW }\end{array}$ & $1-4$ & - & 10 & $0-70 \mathrm{~cm}$ \\
\hline GO & $\begin{array}{c}25.04 .1996 \\
17.04 .1997\end{array}$ & $\begin{array}{l}\text { LBW tief und } \\
\text { LBW flach }\end{array}$ & $1-4$ & - & 10 & $0-70 \mathrm{~cm}$ \\
\hline
\end{tabular}




\subsubsection{Gasdiffusion}

Probenahme

Zur Messung der Gasdiffusion durch den wasserfreien Porenraum des Bodens wurden Stechzylinderproben mit einem Volumen von $200 \mathrm{~cm}^{3}$ aus vertikaler Einschlagrichtung entnommen. Die Beprobungstiefe wurde unterhalb der lokkernden Wirkung des Pfluges (Pflugtiefe max. $25 \mathrm{~cm}$ ) festgelegt, um eine natürliche Gefügeregeneration ohne mechanische Auflockerung erfassen zu können. Die Bodenproben wurden bei $4-6^{\circ} \mathrm{C}$ bis zur Untersuchung gelagert. Tab. 11 faßt die Daten der Probenahme zu Gasdiffusion zusammen.

Tab. 11: Beprobungsplan Gasdiffusion

\begin{tabular}{|c|c|c|c|c|c|c|}
\hline Standort & Bt & Bo & Bs & BI & $\mathbf{n}$ & $\mathbf{T}$ \\
\hline $\mathbf{R H}$ & \begin{tabular}{|l|}
12.04 .1995 \\
16.04 .1997
\end{tabular} & LBW und FMW & $1-4$ & Block 2 & 6 & $28-34$ \\
\hline $\mathbf{R L}$ & 26.04.1995 & LBW und FMW & $1-4$ & - & 6 & $28-34$ \\
\hline GO & 24.03.1995 & $\begin{array}{l}\text { LBW tief und } \\
\text { LBW flach }\end{array}$ & $1-4$ & - & 6 & $28-34$ \\
\hline
\end{tabular}

\section{Meßprinzip}

Die $200 \mathrm{~cm}^{3}$ - Stechzylinderproben wurden nach der Einstellung einer definierten Wasserspannung ( $\mathrm{pF} 1,0,1,7$ und 2,0) mittels keramischer Platten (SCHLICHTING \& BLUME 1966) in Diffusionskammern fixiert. Bei einer Wasserspannung von $\mathrm{pF} 1,0$ sind Poren mit einem Äquivalenzdurchmesser größer als $300 \mu \mathrm{m}$ luftgefüllt. Bei pF 1,7 sind Poren mit einem Äquivalenzdurchmesser von mehr als $50 \mu \mathrm{m}$ luftgefüllt, bei pF 2,0 Poren größer $30 \mu \mathrm{m}$. Durch eine Spülung dieser Kammern mit $\mathrm{N}_{2}$-Gas wurde ein $\mathrm{O}_{2}$-Konzentrationsgefälle gegenüber der Außenluft geschaffen. Entlang dieses Gefälles diffundierte Sauerstoff aus der Raumluft über die luftführenden Poren der Bodenprobe in die Diffusionskammern hinein. Über einen Zeitraum von vier Stunden und bei einer konstanten Raumtemperatur von $20^{\circ} \mathrm{C}$ wurde im Abstand von 30 Minuten die $\mathrm{O}_{2}$ Konzentration in den Diffusionskammern gaschromatographisch festgehalten. 
Berechnung des relativen scheinbaren Gasdiffusionskoeffizienten

Nach WeNDROTH (1990) wurde aus dem Anstieg der $\mathrm{O}_{2}$-Konzentration in den Diffusionskammern der relative scheinbare Gasdiffusionskoeffizient (Ds/Do) als Funktion des luftführenden Porenraumes berechnet. Ds bezeichnet den scheinbaren Diffusionskoeffizienten $\left[\mathrm{cm}^{2} / \mathrm{s}\right]$. Do ist der Diffusionskoeffizient von Sauerstoff in Luft und beträgt 0,203 cm²/s (BAKKER \& HIDDING 1970).

\subsubsection{Ungesättigte Wasserleitfähigkeit}

Probenahme

Zur Probenahme wurden $300 \mathrm{~cm}^{3}$ Stechzylinder mit vertikaler Einschlagrichtung eingesetzt. So wie zur Messung der Gasdiffusion wurde auch zur Bestimmung der ungesättigten Wasserleitfähigkeit die Beprobungstiefe unterhalb der lokkernden Wirkung des Pfluges festgelegt. Eine Zusammenfassung der Beprobungsdaten ist in Tab. 12 zu finden.

Tab. 12: Beprobungsplan ungesättigte Wasserleitfähigkeit

\begin{tabular}{c|cccccc} 
Standort & $\mathbf{B t}$ & Bo & Bs & BI & $\mathbf{n}$ & $\mathbf{T}$ \\
\hline $\mathbf{R H}$ & 12.04 .1995 & LBW und FMW & $1-4$ & Block 2 & 4 & $28-34$ \\
$\mathbf{R L}$ & 26.04 .1995 & LBW und FMW & $1-4$ & - & 4 & $28-34$ \\
GO & 24.03 .1995 & $\begin{array}{c}\text { LBW tief und } \\
\text { LBW flach }\end{array}$ & $1-4$ & - & 4 & $28-34$
\end{tabular}

\section{Meßprinzip}

Die ungesättigte hydraulische Leitfähigkeit (ku) wurde im Porenbereich $<300 \mu \mathrm{m}$ mit einer langsamen Verdunstungsmethoden ermittelt. In einer basal abgedichteten, nach oben offenen Stechzylinder-Probe (Höhe $6 \mathrm{~cm}$ ) ist in 1,5 und 4,5 cm Höhe jeweils ein Druckaufnehmertensiometer in waagerechter Position installiert. Über die offene Oberseite der Probe verdunstet Wasser. Im Zeitverlauf wird der Wasserverlust durch Wägung bestimmt. Gleichzeitig werden die Matrixpotentialwerte am oberen und unteren Tensiometer registriert. Aus den Wertepaaren des Gradienten des Matrixpotentials im SZ und des 
Wasserverlustes der Probe zwischen zwei Meßzeitpunkten wurde nach VAN GENUCHTEN (1980) die pF-Kurve des Bodens berechnet. Nach Wösten und VAN GENUCHTEN (1988) konnte anschließend die ku-Matrixpotential-Funktion berechnet werden. Die benötigten Fitparameter wurden mit dem Programm RETC (WösteN und VAN GenUCHTEN 1988) ermittelt. Eine genaue Beschreibung des Aufbaus der Meßapparatur und der Methode der Messung ist bei WeNDROTH (1990) zu finden.

\subsection{Bestandesentwicklung}

\subsubsection{Pflanzendichte}

Tab. 13 zeigt die Daten der Untersuchung zur Pflanzendichte.

Tab. 13: Beprobungsplan Pflanzendichte; Die Abkürzung „m“ bezeichnet die Meßwiederholungen pro Parzelle x Länge der ausgezählten Pflanzenreihe einer Messung. (Termine zu Aussaat und Feldaufgang siehe Tab. 57-59)

\begin{tabular}{|c|c|c|c|c|c|}
\hline Standort & Bt & Bo & Bs & BI & $\mathbf{m}$ \\
\hline RH & $\begin{array}{l}18.05 .1995 \\
22.11 .1995 \\
29.10 .1996\end{array}$ & $\begin{array}{l}\text { LBW und } \\
\text { FMW }\end{array}$ & $1-4$ & Block 1-4 & $10 \times 1 \mathrm{~m}$ \\
\hline $\mathbf{R L}$ & $\begin{array}{l}11.05 .1995 \\
23.11 .1995 \\
04.03 .1997\end{array}$ & $\begin{array}{l}\text { LBW und } \\
\text { FMW }\end{array}$ & $1-4$ & - & $10 \times 1 \mathrm{~m}$ \\
\hline GO & $\begin{array}{l}26.04 .1995 \\
09.04 .1996 \\
28.10 .1996\end{array}$ & $\begin{array}{c}\text { LBW tief } \\
\text { und LBW } \\
\text { flach }\end{array}$ & $1-4$ & - & $10 \times 1 \mathrm{~m}$ \\
\hline
\end{tabular}

\subsubsection{Wurzelsystem}

\subsubsection{Profilwandmethode}

Diese Methode wurde in Reinshof und Relliehausen benutzt.

An einer $1 \mathrm{~m} \times 1 \mathrm{~m}$ großen Profilwand wurde eine $0,5 \mathrm{~cm}$ dicke Bodenschicht abgewaschen und alle freigespülten Wurzeln in Wurzellängeneinheiten von 0,5 $\mathrm{cm}$ Länge ausgezählt. Die Profilwand war in ein Raster von $5 \mathrm{~cm} \times 5 \mathrm{~cm}$ Kan- 
tenlänge eingeteilt. Das Verhältnis gezählter Wurzellänge zum Volumen des abgespülten Bodens ergibt die Wurzellängendichte (WLD). Sie gilt als Maß für die Durchwurzelungsintensität. In Reinshof wurde 1995 die Wurzellängendichte in der Bodenmatrix und in Bioporen getrennt erfaßt (Tab. 50, 51, Anhang). Die gezählte WLD wurde nicht mit einem Korrekturfaktor verrechnet (KÖPKE 1979).

Die Gesamtwurzellänge (GWL) gibt die aus der WLD einzelner Bodenschichten errechnete Gesamtlänge des Wurzelsystems unter einem $\mathrm{m}^{2}$ Boden bis zur maximalen Durchwurzelungstiefe an. Als maximale Durchwurzelungstiefe wurde die Profilmächtigkeit definiert, in der 99 \% der Gesamtwurzellänge gefunden wurden.

Die Darstellung eines Wurzelprofils zeigt eine Wurzellängeneinheit $(0,5 \mathrm{~cm})$ als einen Punkt. An der Punktdichte und der Punktverteilung ist die Durchwurzelungsdichte und die Wurzelverteilung abzulesen. Auf dem Standort Reinshof wurde die Untersuchung des Wurzelsystems ebenso wie die Messungen zur oberirdischen Trockenmasse und der Blattfläche auf Block 2 durchgeführt.

Tab. 14 zeigt eine Zusammenfassung der Beprobungsdaten zur Untersuchung des Wurzelsystems.

\subsubsection{Bruchflächenmethode}

Diese Methode wurde von der AG Jena in Großobringen eingesetzt.

Die Anzahl Wurzelpassagen pro Fläche wurde mit der Bruchflächenmethode nach Vetter \& Scharafat (1964) erfaßt (Tab. 14). Mit Hilfe eines Metallrahmens (10 cm Kantenlänge, $5 \mathrm{~cm}$ Rahmenhöhe) wurde ein Bodenblock aus dem Boden gebrochen. An der Bruchfläche des Stechzylinders wurden die Wurzelpassagen ausgezählt und auf eine Fläche von $1 \mathrm{~m}^{2}$ umgerechnet. Der Wert Wurzelpassagen pro $\mathrm{m}^{2}$ gibt an, wieviele Wurzeln eine Ebene mit der Fläche von einem $\mathrm{m}^{2}$ in der gemessenen Bodentiefe durchbrechen. Um von der Durchwurzelungshäufigkeit einer Fläche auf die WLD eines Bodenvolumens umrechnen zu können, wurden folgende Annahmen gemacht: In der Bodenschicht $0,5 \mathrm{~cm}$ über, und der Schicht $0,5 \mathrm{~cm}$ unter der ausgezählten Fläche verzweigen sich die Wurzeln nicht und durchwachsen den Bodenhorizont vertikal. Einer Wurzelpassage kann so eine Wurzellänge von $1 \mathrm{~cm}$ Höhe zugeordnet werden. Aus der Wurzellänge in der Bodenschicht mit den Ausmaßen $100 \mathrm{~cm}$ x 
$100 \mathrm{~cm} \times 1 \mathrm{~cm}$ läßt sich unschwer die WLD in $\mathrm{cm}$ pro $\mathrm{cm}^{3}$ errechnen. Die so berechnete WLD wird als Annäherung an die tatsächliche Durchwurzelungsintensität in der untersuchten Bodentiefe angesehen. Um ein Maß für die Größe des Wurzelsystems zu erlangen, wurde die WLD der nicht untersuchten Bodenschichten auf der Grundlage des vorliegenden Datenmaterials nach folgendem Muster interpoliert: Die WLD der Messung bei $10 \mathrm{~cm}$ Bodentiefe wurde als WLD der Bodenschicht $0-15 \mathrm{~cm}$ angenommen, die Werte der Messung bei 20 $\mathrm{cm}$ für die Schicht $15-30 \mathrm{~cm}$, die Werte bei $35 \mathrm{~cm}$ für die Schicht $30-40 \mathrm{~cm}$ und die Werte bei $50 \mathrm{~cm}$ für die Schicht $40-50 \mathrm{~cm}$. Aus der Summe der WLD der einzelnen Bodenschichten ergibt sich die GWL bis $50 \mathrm{~cm}$ Bodentiefe. Aussagen zur maximalen Durchwurzelungstiefe können nicht gemacht werden.

Tab. 14: Beprobungsplan Wurzelsystem.

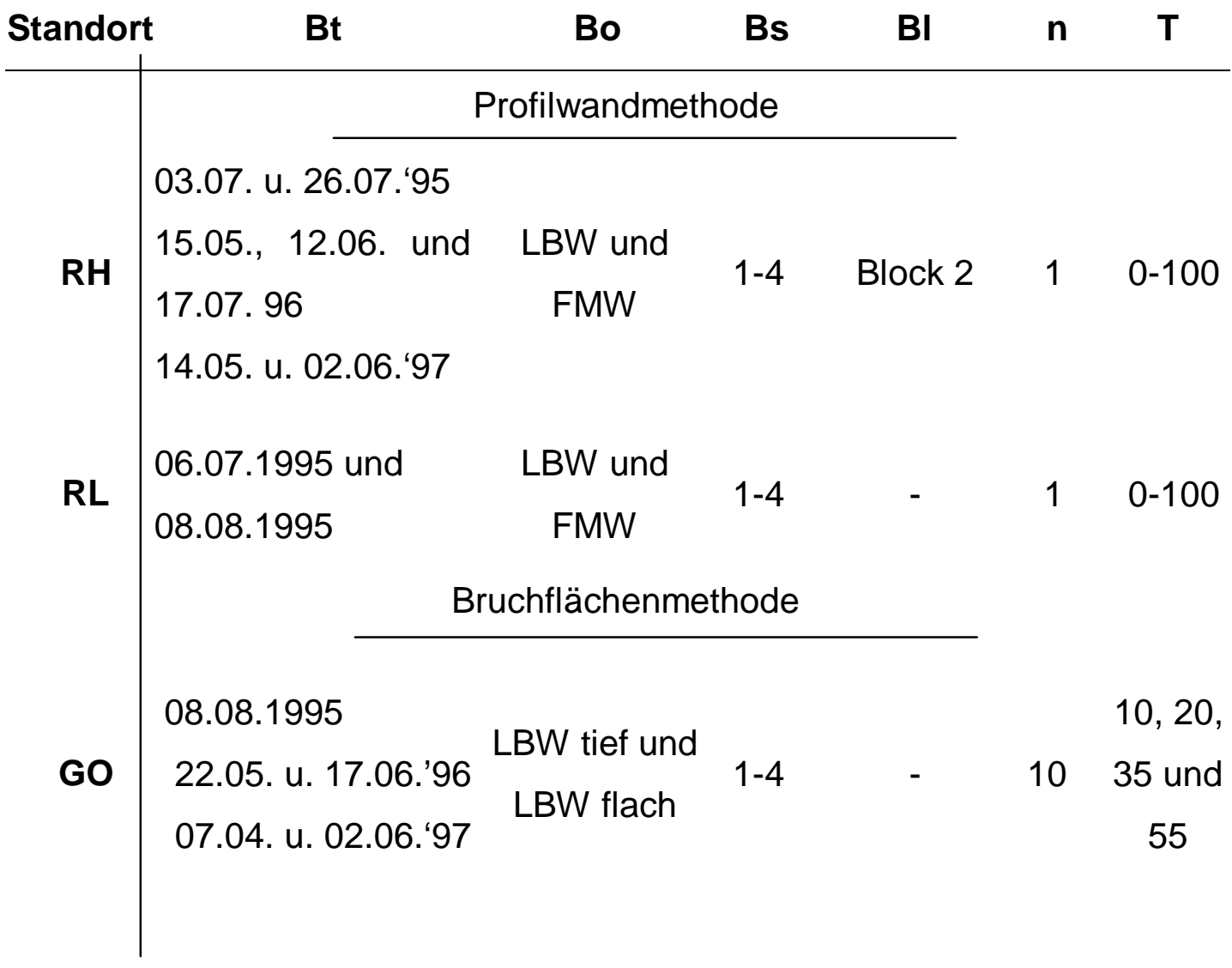

\subsubsection{Wurzeldurchmesser}

Im Versuchsjahr 1996 wurde auf dem Standort RH der Wurzeldurchmesser des Winterweizens in drei Bodentiefen bei LBW und FMW mit 0t und 6x5t auf Block 2 erfaßt. Eine Beschreibung der Methode findet sich bei MERTEN (1997). 


\subsubsection{Oberirdische Trockenmasse}

Pro Parzelle wurden Teilernten auf einer Fläche von $1 \mathrm{~m}^{2}$ durchgeführt. Die Daten zur Probenahme sind in Tab. 15 und 16 zusammengefaßt.

Tab. 15: Beprobungstermine (Bt) der Teilernten zur Bestimmung der oberirdischen Biomasse und der Blattfläche. Neben dem Versuchsjahr sind die angebauten Feldfrüchte Sommergerste (SG), Winterweizen (WW) und Wintergerste (WG) angegeben.

Versuchsjahr und Feldfrucht

\begin{tabular}{|c|c|c|c|}
\hline Standort & 1995 (SG) & 1996 (WW) & 1997 (WG) \\
\hline $\mathbf{R H}$ & $\begin{array}{l}30.05 \\
13.06 \\
29.06\end{array}$ & $\begin{array}{l}22.04 \\
08.05 \\
22.05 \\
05.06 \\
19.06 \\
03.07 \\
18.07 \\
01.08\end{array}$ & $\begin{array}{l}23.04 . \\
07.05 \\
22.05 \\
26.05 \\
04.06 \\
16.06 \\
25.06\end{array}$ \\
\hline RL & $\begin{array}{l}06.06 \\
21.06 \\
01.07\end{array}$ & $\begin{array}{l}03.05 \\
02.06 \\
26.06 \\
27.07\end{array}$ & - \\
\hline GO & - & $\begin{array}{l}25.04 \\
09.05 \\
23.05 \\
06.06 \\
20.06 \\
04.07 \\
18.07 \\
01.08 \\
15.08\end{array}$ & $\begin{array}{l}14.04 . \\
29.04 . \\
12.05 . \\
26.05 . \\
09.06 . \\
26.06 .\end{array}$ \\
\hline
\end{tabular}

Die Lage der Ernteflächen in den Parzellen wurden so gewählt, daß sie - aneinander anschließend - über die gesamte Vegetationsdauer den gleichen Pflanzenreihen folgten. Zu den Ernten wurde der gesamte oberirdische Aufwuchs der Erntefläche flach über dem Boden abgetrennt. Pflanzenteile, die nicht von den Kulturpflanzen stammten, wurden ebenso wie Strohreste und Verunreinigungen beseitigt. An dem so gewonnenen Erntegut wurde neben der oberirdischen Biomasse auch die Blattfläche bestimmt. Zur Trockenmassebestimmung wurde ein Aliquot $24 \mathrm{~h}$ bei $65^{\circ} \mathrm{C}$ und anschließend $24 \mathrm{~h}$ bei $105^{\circ} \mathrm{C}$ bis zur Ge- 
wichtskonstanz getrocknet. Pro Parzelle wurde pro Termin eine Probe gewonnen.

\subsubsection{Blattfläche}

Bei allen Teilernten wurde die Fläche der Blattspreiten der Kulturpflanzen an einem Aliquot mit einem Blattflächenmeßgerät des Typs Cl-203 der Firma CID, Inc., Vancouver, U.S.A ermittelt. Der Blattflächenindex gibt die Gesamtfläche der Blattspreiten in $\mathrm{m}^{2}$ pro $\mathrm{m}^{2}$ Bodenoberfläche an. Tab. 15 und 16 zeigen den Beprobungsplan.

Tab. 16: Beprobungsplan oberirdische Biomasse und Blattfäche.

\begin{tabular}{|c|c|c|c|c|}
\hline Standort & Bo & Bs & BI & $\mathbf{n}$ \\
\hline $\mathbf{R H}$ & LBW und FMW & $1-4$ & $\begin{array}{c}\text { Block } 2(1995 / 96) \\
\text { Block } 3(1997)\end{array}$ & 1 \\
\hline $\mathbf{R L}$ & LBW und FMW & $1-4$ & - & 1 \\
\hline GO & $\begin{array}{l}\text { LBW tief und LBW } \\
\text { flach }\end{array}$ & $1-4$ & - & 1 \\
\hline
\end{tabular}

\subsubsection{Bestandeshöhe}

Die Bestandeshöhe wurde nicht an Einzelpflanzen erfaßt, sondern an ca. $25 \mathrm{~m}^{2}$ großen Teilflächen. Die mittlere Bestandeshöhe einer Teilfläche wurde aus ca. $5 \mathrm{~m}$ Entfernung geschätzt, indem die ablesende Person genau über den Bestand einen Maßstab anvisierte. Tab. 17 faßt die Untersuchungsdaten zusammen. 
Tab. 17: Beprobungsplan Bestandeshöhe

\begin{tabular}{c|ccccc} 
Standort & $\mathbf{B t}$ & Bo & Bs & BI & $\mathbf{n}$ \\
\hline \multirow{2}{*}{$\mathbf{R H}$} & $\begin{array}{c}\text { 27.05. u. 28.06.'95 } \\
\text { 19.05. u. 17.06.'96 }\end{array}$ & LBW und & & & \\
& 22.04. u. 26.05.97 & FMW & $1-4$ & Block 1-4 & 10 \\
$\mathbf{R L}$ & 02.06. u. 26.06.96 & LBW und FMW & $1-4$ & - & 3 \\
& 08.08. 1995 & LBW tief und & & & \\
GO & 09.09.1996 & LBW flach & $1-4$ & - & 10 \\
& 15.07 .1997 & & & &
\end{tabular}

\subsubsection{Fahnenblattlänge}

Die Länge eines Fahnenblattes wurde vom Blatthäutchen bis zur Spitze der Blattspreite bestimmt. Gemessen wurde an nicht abgetrennten Blättern lebender Pflanzen im Bestand. Die Daten der Messungen zur Fähnenblattlänge sind in Tab. 18 zusammengefaßt.

Tab. 18: Beprobungsplan Fahnenblattlänge

\begin{tabular}{c|ccccc} 
Standort & Bt & Bo & Bs & Bl & $\mathbf{n}$ \\
\hline $\mathbf{R H}$ & 18.06 .1997 & $\begin{array}{c}\text { LBW und } \\
\text { FMW }\end{array}$ & $1-4$ & Block 1-4 & 30
\end{tabular}

\subsubsection{Wachstumsstadien}

Die Entwicklungsstadien der Kulturpflanzen wurden nach ZADOKS et al. (1974) bestimmt. Tab. 40 im Anhang zeigt die Einteilung des Getreidewachstums nach ZADOKS et al. (1974).

\subsubsection{Gesamtwasserpotential}

Zur Messung des Gesamtwasserpotentials (GWP) der Kulturpflanzen wurde eine Druckkammer der Firma pms instrument corp., Oregon, U.S.A. eingesetzt. Die Technik, das GWP mit Hilfe einer Druckkammer zu erfassen, wurde grundlegend von SCHOLANDER et al. (1965) entwickelt. Eine genaue Beschreibung der 
Messung des GWP mit Hilfe der Druckapparatur findet sich bei EHLERS (1996). Das jüngste voll ausgebildete Blatt einer Pflanze wurde abgetrennt und zur Messung in die Druckkammer eingespannt. Die Zeitspanne von der Probenahme bis zum Beginn der Messung betrug maximal 40 s. Die Druckzunahme wurde auf 0,2 bar/s festgelegt. Tab. 19 zeigt eine Zusammenfassung der Beprobungsdaten zur Untersuchung des GWP.

Tab. 19: Beprobungsplan Gesamtwasserpotential

Mit dem Beginn einer Messung wurden die Belastungsstufen nach dem Muster FMW Stufe 4 bis1, dann LBW Stufe 1 bis 4 in nicht regelmäßigen Abständen fortlaufend bis zum Ende der Messung beprobt.

\begin{tabular}{|c|c|c|c|c|}
\hline Standort & Bt & Bo & Bs & BI \\
\hline \multirow{8}{*}{ RH } & $23.06 .1996(07: 00-18: 00)$ & \multirow{8}{*}{$\begin{array}{c}\text { LBW und } \\
\text { FMW }\end{array}$} & \multirow{8}{*}{$1-4$} & Block 2 \\
\hline & 04.06.1997 (09:00-09:40) & & & (1996) \\
\hline & 05.06.1997 (08:40-16:50) & & & Block 3 \\
\hline & 11.06.1997 (08:35-14:30) & & & (1997) \\
\hline & 12.06.1997 (04:10-13:30) & & & \\
\hline & 17.06.1997 (12:05-15:40) & & & \\
\hline & 18.06.1997 (04:10-08:00) & & & \\
\hline & 19.06.1997 (08:00-15:40) & & & \\
\hline
\end{tabular}

\subsubsection{Gaswechsel der Kulturpflanzen}

Der $\mathrm{CO}_{2} / \mathrm{H}_{2} \mathrm{O}$ Gaswechsel der Kulturpflanzen und die Strahlungsintensität wurden zeitgleich mit einem tragbaren Porometer des Typs CIRAS S/N 110 (Combined Infrared Gas Analysis System) der Firma PP-Systems, Großbritannien gemessen. $\mathrm{Zu}$ jeder Einzelmessung wurde das jüngste voll entwickelte Blatt einer Pflanze in eine Küvette von $4,5 \mathrm{~cm}^{2}$ Fläche (Typ PLC N, PPSystems, Groß Britannien) eingespannt. In der Küvette wurde ein Luftstrom mit einer Flußrate von $5 \mathrm{~cm}^{3} / \mathrm{s}$ bei $20^{\circ} \mathrm{C}$ und 1 bar Luftdruck an der Blattfläche vorbeigeführt. Die $\mathrm{CO}_{2}$ - Konzentration und der Wassergehalt des eingehenden Luftstroms waren festgelegt auf 350 ppm $\mathrm{CO}_{2}$ und 99\% der umgebenden Luftfeuchtigkeit zum Zeitpunkt der Messung. Die photosynthetisch aktive Strahlung (PAR) zwischen 400 und 700 nm erfaßte ein Punktsensor an der Küvette. Aus 
der Lufttemperatur wurde nach der MAGNUSSCHEN Formel (WEISCHET 1995) der Sättigungsdampfdruck errechnet. Die Differenz des Sättigungsdampfdrukkes und des aktuellen Wasserdampfdruckes ergab das Sättigungsdefizit. Da der Gaswechsel und das GWP der Pflanzen auch bei hohem Wasserstreß ermittelt werden sollte, wurden die Messungen nur an warmen, strahlungsreichen Tagen mit einer geringen Wolkendecke in einer Trockenperiode ohne Niederschlag durchgeführt. Bei aufziehender Bewölkung wurden die Messungen abgebrochen. Wenn möglich wurde der Tagesgang erfaßt. Der Beprobungsplan für die Gaswechselmessung findet sich in Tab. 20.

Tab. 20: Beprobungsplan $\mathrm{CO}_{2} / \mathrm{H}_{2} \mathrm{O}-$ Gaswechsel

Mit dem Beginn einer Messung wurden die Belastungsstufen nach dem Muster FMW Stufe 4 bis1, dann LBW Stufe 1 bis 4 in nicht regelmäßigen Abständen fortlaufend beprobt.

\begin{tabular}{c|cccc} 
Standort & Bt & Bo & Bs & BI \\
\hline \multirow{6}{*}{ RH } & $\begin{array}{l}\text { 23.06.1996 (07:00-18:00) } \\
\text { 04.06.1997 (09:00-09:40) }\end{array}$ & & & Block 2 \\
$05.06 .1997(08: 40-16: 50)$ & LBW & & \\
$11.06 .1997(08: 35-14: 30)$ & und & $1-4$ & Block 3 \\
$12.06 .1997(04: 10-13: 30)$ & FMW & & $(1997)$ \\
$17.06 .1997(12: 05-15: 40)$ & & & \\
$18.06 .1997(04: 10-08: 00)$ & & & \\
$19.06 .1997(08: 00-15: 40)$ & & &
\end{tabular}

\subsubsection{Ableitung von Gaswechselkenngrößen}

Die folgenden Kenngrößen wurden vom internen Rechner des Porometers berechnet.

\subsubsection{1 $\quad \mathrm{CO}_{2}-$ Assimilationsrate}

Die $\mathrm{CO}_{2}-$ Assimilationsrate ergab sich aus der Differenz der $\mathrm{CO}_{2}$ Konzentration der in die Küvette einströmenden Luft und der aus der Küvette austretenden 
Luft. Die $\mathrm{CO}_{2}-$ Assimilationsrate gibt an, wieviel $\mathrm{CO}_{2}[\mu \mathrm{mol}]$ pro Blattfläche $\left[\mathrm{m}^{2}\right]$ und pro Zeiteinheit [s] aufgenommen wurde.

\subsection{Transpirationsrate}

Aus dem Wasserdampfdruck der in die Küvette einströmenden Luft und dem Wasserdampfdruck der austretenden Luft wurde die Menge an Wasser ermittelt, welche die Blattfläche in der Küvette während der Messung abgab. Die Transpirationsrate gibt an, wieviel Wasser [mmol] pro Blattfläche $\left[\mathrm{m}^{2}\right]$ und pro Zeiteinheit [s] aus dem Blattinneren in die umgebende Luft diffundierte.

\subsection{Stomatäre Leitfähigkeit für Wasserdampf}

Aus der Transpirationsrate und dem Grenzflächenwiderstand für Wasserdampf an der Blattoberfläche wurde die stomatäre Leitfähigkeit für Wasserdampf der eingespannten Blattfläche berechnet. Diese Vorgehensweise setzte voraus, daß der Grenzflächenwiderstand an Blattober- und Blattunterseite gleich war. Eine weitere Annahme war die gleichmäßige Verteilung der Stomata auf beiden Seiten des Blattes. Die stomatäre Leitfähigkeit für Wasserdampf [mmol $\left.\mathrm{H}_{2} \mathrm{O} / \mathrm{m}^{2} / \mathrm{s}\right]$ ist ein $\mathrm{Maß}$ für die Öffnungsweite der Stomata. Bei dieser Betrachtungsweise wurde die kutikuläre Wasserabgabe nicht berücksichtigt.

\subsection{Untersuchungen der Ertragsbildung und des Ertrages}

3.4.1 Ährendichte und Kornbesatz der Ähre

Die Daten zur Untersuchung der Ährendichte und des Kornbesatzes der Ähre sind in Tab. 21 dargestellt. 
Tab. 21: Beprobungsplan Ährendichte und Kornbesatz der Ähre;

Die Abkürzung „n“ bezeichnet die Meßwiederholungen pro Parzelle x Länge der untersuchten Pflanzenreihe einer Messung.

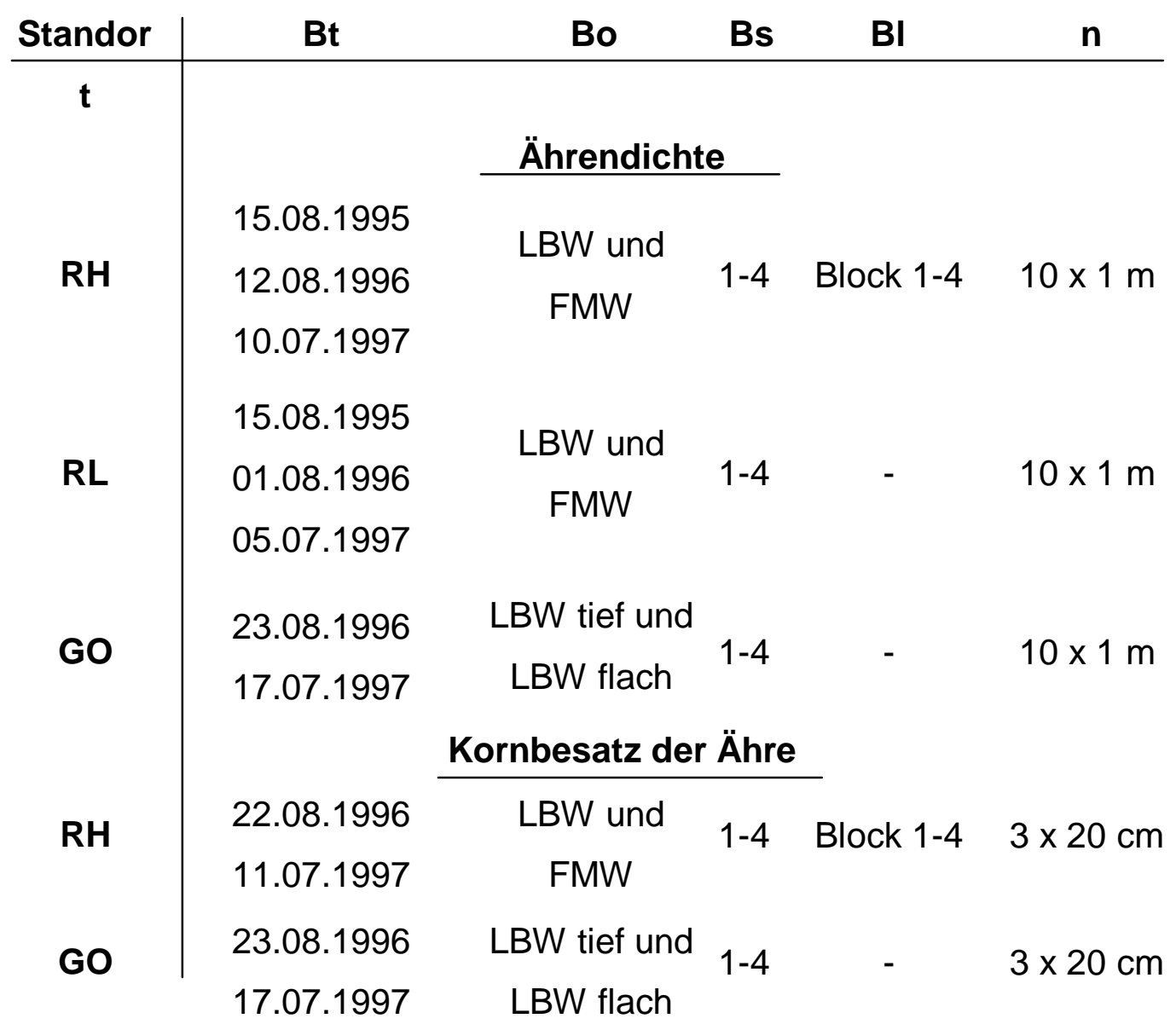

\subsubsection{Korn- und Strohertrag}

Das von Hand gemähte Erntegut wurde auf allen Standorten mit einem stationär betriebenen Parzellenmähdrescher in Stroh und Korn geteilt. Anschließend wurde das Frischgewicht der Fraktionen festgehalten. Teilproben (Stroh: 2x100g Frischmasse, Korn 2x200g Frischmasse) dienten zur Feststellung des Trockengewichtes. Alle Trocknungsvorgänge liefen bis zur Gewichtskonstanz 24 Std bei $60^{\circ} \mathrm{C}$ und anschließend 24 Std bei $105^{\circ} \mathrm{C}$. Die geernteten Mengen wurden gemittelt und umgerechnet auf dt Trockenmasse/ha. Angegeben wird der gereinigte Kornertrag. Die Beprobungsdaten der Ernten zeigt Tab. 22. 
Tab. 22: Beprobungsplan Korn- und Strohertrag

\begin{tabular}{|c|c|c|c|c|c|}
\hline Standort & Bt & Bo & Bs & BI & $\mathbf{n}$ \\
\hline $\mathbf{R H}$ & $\begin{array}{l}15.08 .1995 \\
23.08 .1996\end{array}$ & $\begin{array}{l}\text { LBW und } \\
\text { FMW }\end{array}$ & $1-4$ & Block 1-4 & $4 \times 1 \mathrm{~m}^{2}$ \\
\hline $\mathbf{R H}$ & 22.07.1997 & $\begin{array}{l}\text { LBW und } \\
\text { FMW }\end{array}$ & $1-4$ & Block 1-4 & $8 \times 1 \mathrm{~m}^{2}$ \\
\hline $\mathbf{R L}$ & $\begin{array}{l}15.08 .1995 \\
01.08 .1996 \\
05.07 .1997\end{array}$ & $\begin{array}{l}\text { LBW und } \\
\text { FMW }\end{array}$ & $1-4$ & - & $4 \times 1 \mathrm{~m}^{2}$ \\
\hline GO & 14.06.1995 & $\begin{array}{l}\text { LBW tief und } \\
\text { LBW flach }\end{array}$ & $1-4$ & - & $5 \times 1 \mathrm{~m}^{2}$ \\
\hline GO & $\begin{array}{l}23.08 .1996 \\
17.07 .1997\end{array}$ & $\begin{array}{l}\text { LBW tief und } \\
\text { LBW flach }\end{array}$ & $1-4$ & - & $10 \times 1 \mathrm{~m}^{2}$ \\
\hline
\end{tabular}

\subsubsection{Einzelkornmasse}

Aus dem gereinigten Erntegut der Parzellen wurden 8 Proben von je 100 ganzen Körnern bis zur Gewichtskonstanz getrocknet. Zur Trockenmassebestimmung wurde das Erntegut $24 \mathrm{~h}$ bei $65^{\circ} \mathrm{C}$ und anschließend $24 \mathrm{~h}$ bei $105^{\circ} \mathrm{C}$ bis zur Gewichtskonstanz getrocknet. Die Einzelkornmasse [mg] ergab sich als der hundertste Teil einer Probe. Angegeben wird der Mittelwert aus den 8 Messungen.

\subsubsection{Ernteindex}

Der Ernteindex gibt das Verhältnis des gereinigten Kornertrages (Trockenmasse) zum oberirdischen Gesamtertrag (Trockenmasse) an. Der Gesamtertrag setzt sich zusammen aus dem gereinigten Kornertrag (Trockenmasse) pro Fläche und dem Strohertrag (Trockenmasse) pro Fläche. 


\subsubsection{Berechneter Ertrag}

Auf den Standorten Reinshof und Großobringen wurden in den Versuchsjahren 1996 und 1997 der Kornertrag als Produkt der ertragsbestimmenden Faktoren Ähren pro Flächeneinheit, Körner pro Ähre und Einzelkornmasse bestimmt. Der berechnete Ertrag wird in dt TM pro ha angegeben.

\subsection{Statistische Auswertung}

Statistische Analysen wurden mit Hilfe des SAS-Programms (StatisticalAnalysis-System, SAS-INSTITUT INC. 1989, Version 6.11) nach dem allgemeinen linearen Modell (GLM-Prozedur) durchgeführt. Tests auf Normalverteilung der Residuen wurden mit dem Shapiro-Wilk-Test durchgeführt.

Bei der Analyse von Zeitreihen wurde die 'GLM'-Prozedur um die 'repeated' Anweisung ergänzt. Multiple Vergleiche der Mittelwerte wurden mit Hilfe des Tukey-Tests durchgeführt. Bei allen Berechnungen galt ein Testniveau von $p$ $<5 \%$ als Widerlegung der Nullhypothese. 


\section{Ergebnisse}

\subsection{Bodenphysikalische Eigenschaften}

\subsubsection{Eindringwiderstand}

Die Untersuchung des EDW sollte zeigen, ob die mechanische Bodenbelastung bei den unterschiedlichen Bearbeitungssystemen zu Gefügeveränderungen geführt hat, und wie sich diese Veränderungen im Verlauf der Zeit darstellten. Da mit sinkendem Bodenwassergehalt der EDW eines Bodens zunimmt (EHLERS et al. 1983), wurde die Messung bei oder nahe der Feldkapazität (FK) der Böden jeweils im Frühjahr durchgeführt. Die Werte für den EDW bei geringer und mittlerer Bodenbelastung lagen zwischen den Werten bei keiner und hoher Belastung. Deshalb wird auf deren Darstellung hier verzichtet. Abb. 7 und 8 zeigen den EDW dargestellt als Funktion der Bodentiefe auf den Standorten Reinshof und Relliehausen.

Die EDW - Tiefenfunktionen bei LBW 0t (Abb.7 und 8, links oben) zeigen auf beiden Standorten einen für gepflügte Böden typischen Verlauf: Im Bearbeitungshorizont ist der EDW gering. Im Bereich der Pflugsohle nimmt er sprunghaft zu. Unterhalb dieser Verdichtungs- und Verformungszone nimmt der EDW wieder ab. Der Verlauf der Funktionen kann einen Hinweis auf die Stärke und die Mächtigkeit einer Krumenbasisverdichtung (KBV) unterhalb des Bearbeitungshorizontes geben. Auf dem Standort Reinshof lag der EDW bei gepflügtem Boden ohne Belastung (Abb. 7, links oben), gemittelt über die Versuchsjahre, in der Pflugfurche bei ca. 3,0 MPa. Die Mächtigkeit dieses Horizontes mit hohem EDW betrug weniger als $10 \mathrm{~cm}$. 


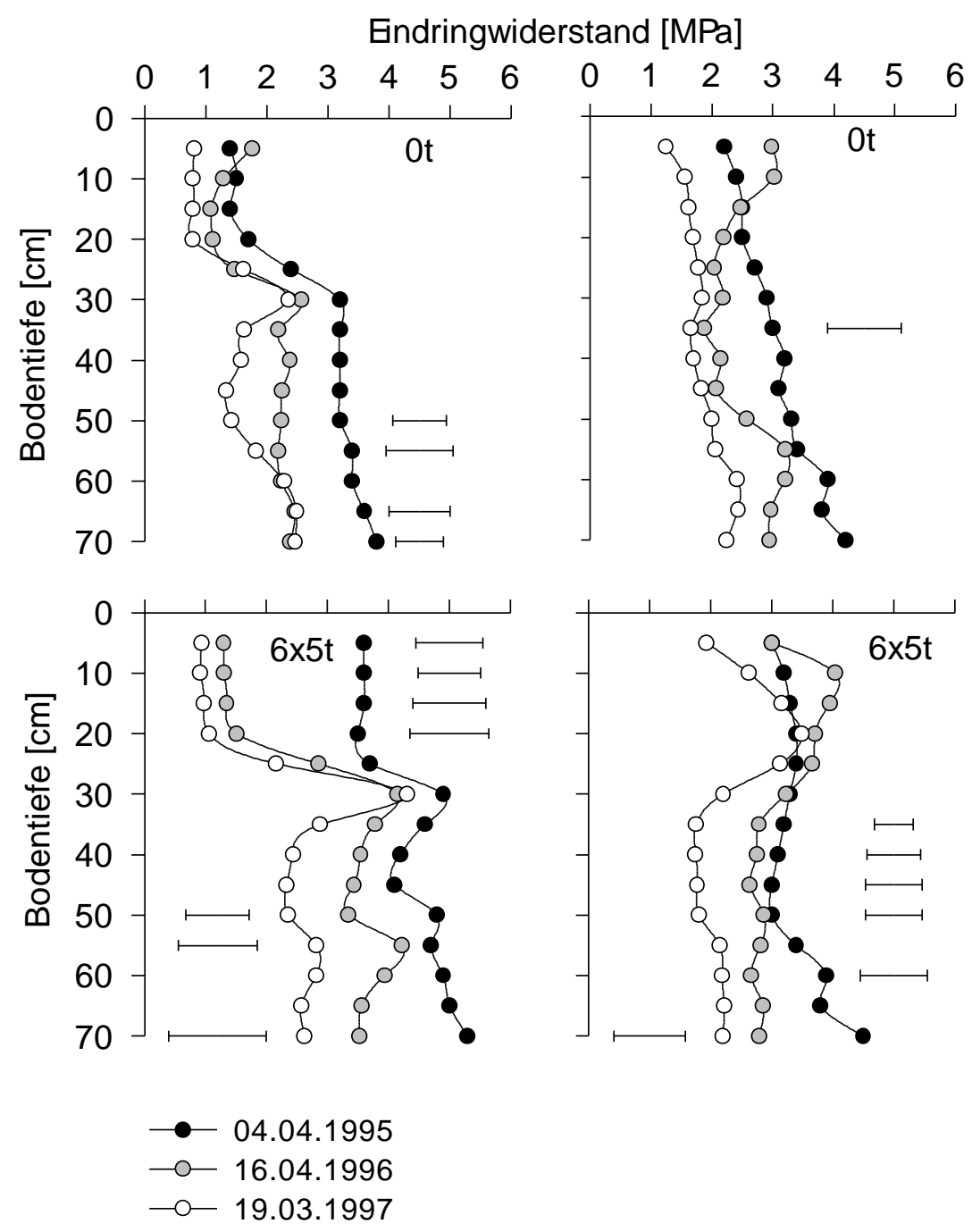

Abb. 7: Eindringwiderstand - Tiefenfunktionen des Standortes Reinshof der Jahre 1995,1996 und 1997. Links dargestellt ist die Lockerbodenwirtschaft (LBW), rechts die Festboden-Mulchwirtschaft (FMW). Die Abkürzungen 0t und 6x5t geben die Überrollhäufigkeit x Radlast an. Aufgetragen sind arithmetische Mittelwerte. Signifikante Unterschiede zwischen den Jahren werden durch horizontale Striche (Grenzdifferenz) dargestellt. 


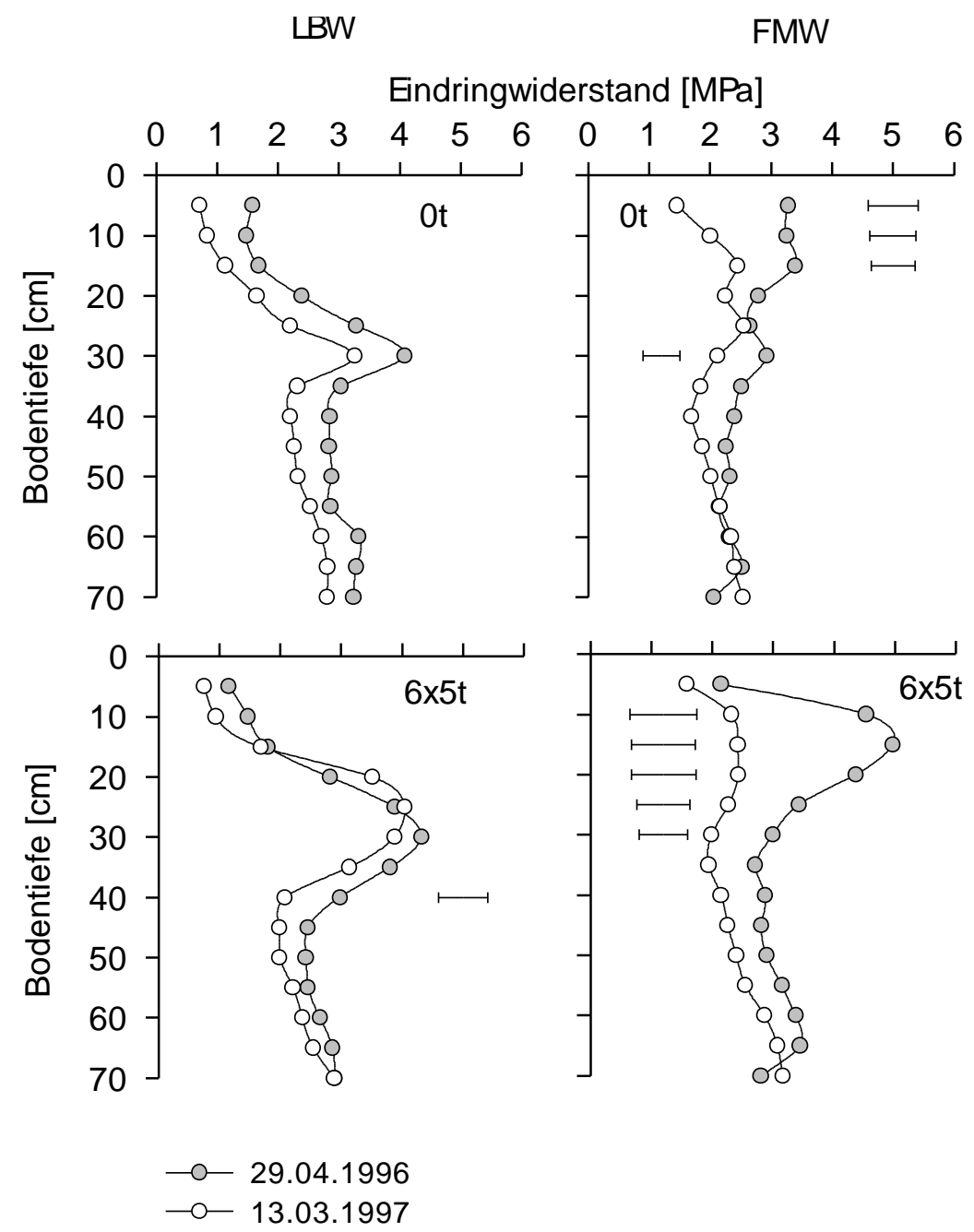

Abb. 8: Eindringwiderstand - Tiefenfunktionen des Standortes Relliehausen der Jahre 1996 und 1997. Aufgetragen sind arithmetische Mittelwerte. Signifikante Unterschied zwischen den Jahren werden durch horizontale Striche (Grenzdifferenz) dargestellt.

In Reinshof führte die hohe Belastung des gepflügten Bodens in der Ackerkrume zu einem Anstieg des EDW um ca. 1,5 MPa (Abb. 7, links unten). Die Mächtigkeit der Verdichtungs- und Verformungszone an der Krumenbasis nahm - verglichen mit dem unbelasteten Boden - durch die hohe Belastung kaum zu. Unterhalb von $40 \mathrm{~cm}$ Bodentiefe lag der EDW auf dem Standort Reinshof bei LBW 6x5t in den drei Versuchsjahren jeweils um ca. $1 \mathrm{MPa}$ höher als bei LBW 0t (Abb.7 links). Auf dem Standort Reinshof stieg bei FMW 0t der EDW - über die drei Versuchsjahre gemittelt - von ca. 1,5 MPa in der obersten Bodenschicht annähernd linear auf etwa 2,5 MPa in $70 \mathrm{~cm}$ Bodentiefe an. Durch die hohe Belastung wurde der EDW in den obersten $30 \mathrm{~cm}$ 
stark erhöht. Die Höchstwerte liegen - über die Jahre gemittelt - bei 3,0 bis 3,5 $\mathrm{MPa}$ (Abb. 7 rechts unten). Unterhalb von $30 \mathrm{~cm}$ Bodentiefe unterschieden sich bei FMW die EDW der Böden mit unterschiedlichen Belastungsstufen nicht.

Betrachtet man den EDW des gepflügten Boden über die Versuchsjahre, so zeigt sich auf dem Standort Reinshof bei hoher Belastung die Wirkung des Pfluges im Bearbeitungshorizont besonders deutlich: Im ersten Versuchsjahr direkt nach der mechanischen Bodenbelastung - lag der EDW im Oberboden mit Werten um 3,5 MPa hoch. Durch die Wirkung des Pfluges wurde der EDW auf Werte um 1,1 MPa in den Jahren 1996 und 1997 gesenkt. So wie der EDW, so nahm auch die Lagerungsdichte des Bodens in der Bodenschicht um $10 \mathrm{~cm}$ Tiefe von 1995 nach 1997 ab (Tab. 41-43, Anhang). Unterhalb von $30 \mathrm{~cm}$ Bodentiefe schien der EDW auf dem gepflügten Boden bei beiden Belastungsstufen über die Versuchsjahre stetig abzunehmen (Abb. 7, links). Die Lagerungsdichten deuteten im Unterboden bei einer Tiefe von $40 \mathrm{~cm}$ keine Veränderung über die Zeit an. Eine tendenzielle Verringerung des EDW im Unterboden von 1995 bis 1997 war nicht nur bei LBW, sondern auch bei FMW festzustellen. Wie Tab. 41-43 im Anhang zeigen, blieben auch hier die Lagerungsdichten im Unterboden (40 cm Tiefe) über die Versuchsjahre annähernd konstant.

In Relliehausen zeichnete sich 1996 die Zone unter dem Bearbeitungshorizont bei LBW 0t mit Maximalwerten um 3,5 MPa und einer Mächtigkeit von ca. $10 \mathrm{~cm}$ aus (Abb. 8 links oben). Maximalwerte des EDW wurden bei LBW 6x5t (Abb. 8, links unten) mit ca. 4,5 MPa gemessen. Die Mächtigkeit der Zone mit hohem EDW unter dem Bearbeitungshorizont lag in etwa bei $15 \mathrm{~cm}$. Die hohe Belastung führte in diesem Horizont zu einer Erhöhung des EDW um ca. 1,0 MPa (Abb. 8 links unten).

In Relliehausen nahm, gemittelt über 1996 und 1997, bei FMW Ot der EDW bis zu einer Bodentiefe von $15-20 \mathrm{~cm}$ bis auf etwa 2,5 MPa zu (Abb. 8 rechts oben). Im Jahr 1996 lag bei FMW mit hoher Belastung der EDW in der Bodentiefe um $15 \mathrm{~cm}$ bei Höchstwerten von 5,0 MPa. Im Jahr 1997 fielen die Werte auf etwa 2,5 MPa zurück (Abb. 8 rechts). Unterhalb von $30 \mathrm{~cm}$ Bodentiefe lag 
der EDW bei allen Belastungsstufen und über die zwei Versuchsjahe mit ca. 2,5 MPa auf gleichem Niveau (Abb. 8 rechts). Wie auf dem Standort Reinshof zeigte sich auch in Relliehausen die Tendenz, daß von einem Jahr zum anderen der EDW der Böden abnahm. Dies zeigte sich bei FMW 6x5t besonders deutlich (Abb. 8 rechts unten).

Bei LBW stieg 1995 die Lagerungsdichte des Bodens in Relliehausen unterhalb der Krumenbasis in der Bodentiefe $30-36 \mathrm{~cm}$ von 1,57 g/cm ohne Belastung auf 1,65 $\mathrm{g} / \mathrm{cm}^{3}$ bei hoher Belastung an (Tab. 44-46, Anhang). Durch die hohe Belastung wurde 1995 die Lagerungsdichte der Krumenbasis stark erhöht. Im Gegensatz zum EDW zeigte die Lagerungsdichte über die Versuchsjahre 1996 und 1997 in der Krumenbasis bei LBW keine kontinuierliche Abnahme (Tab. 44-46, Anhang). Bei FMW 0t wurden in den drei Versuchjahren keine Unterschiede der Lagerungsdichte des Boden über die Tiefe gemessen. Die hohe Belastung führte unterhalb von ca. $10 \mathrm{~cm}$ Bodentiefe zu einem geringfügigen Anstieg der Lagerungsdichte. In Relliehausen zeigte die Lagerungsdichte bei FMW 6x5t über die Versuchsjahre keine deutlichen Unterschiede (Tab. 44-46, Anhang).

Auf dem Standort Großobringen wiesen die EDW keine Unterschiede zwischen den Bearbeitungssystemen, den Belastungsstufen oder den Versuchsjahren auf (Abb. 9 ). Allein auf dem flach gepflügten Boden (Abb. 9 rechts unten) lag der EDW in der Bodenschicht um 15 cm 1997 signifikant höher als im Jahr 1996. Im Gegensatz zu Reinshof und Relliehausen wurden Werte von 2,0 MPa kaum erreicht und überschritten. 


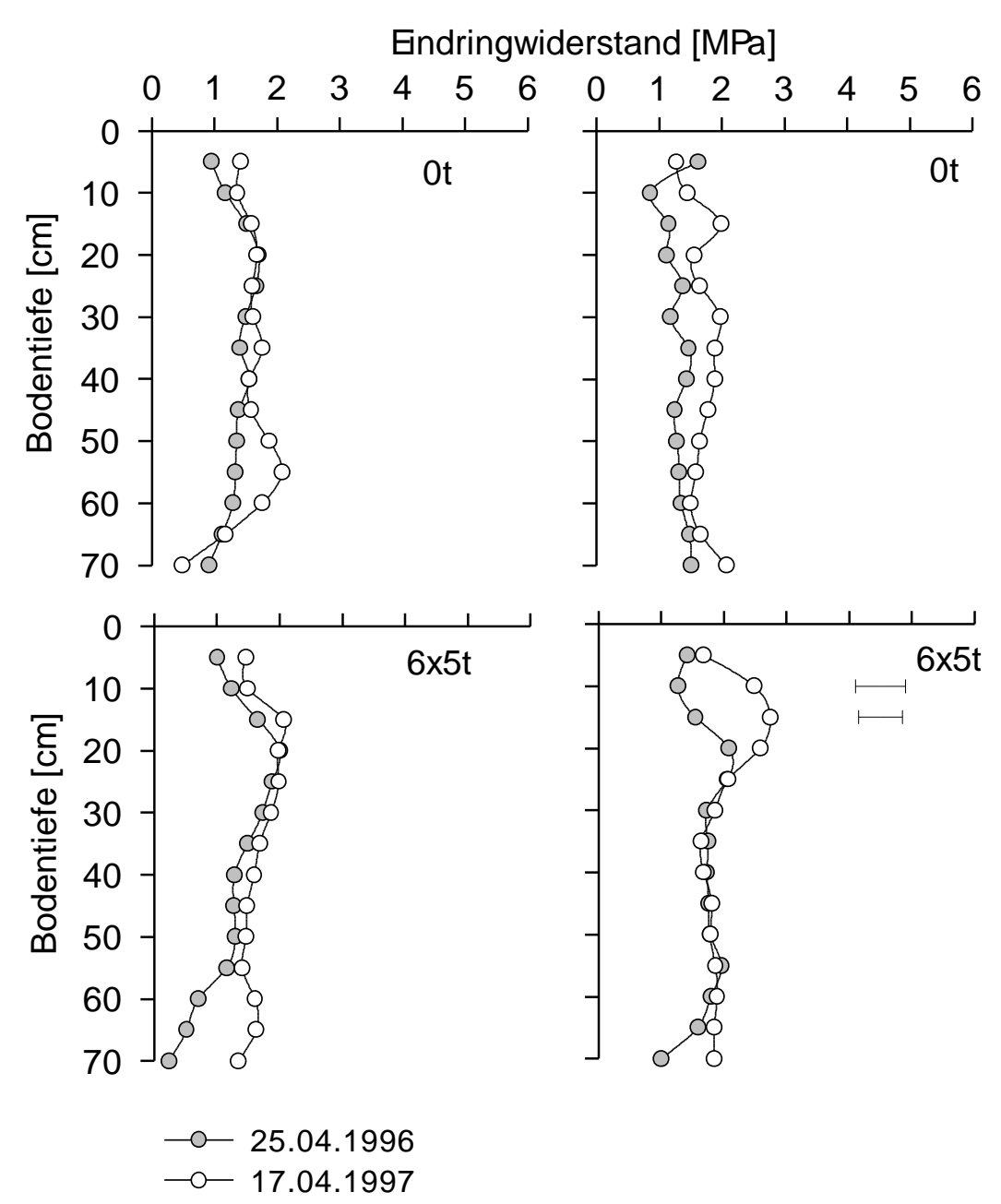

Abb. 9: Eindringwiderstand - Tiefenfunktionen des Standortes Großobringen der Jahre 1996 und 1997. Links dargestellt ist die Lockerbodenwirtschaft mit tiefer Bearbeitung, rechts die Lockerbodenwirtschaft mit flacher Bearbeitung. Aufgetragen sind arithmetische Mittelwerte. Signifikante Unterschiede zwischen den Jahren werden durch horizontale Striche (Grenzdifferenz) dargestellt.

Bei beiden Bearbeitungssystemen lagerte der Boden nach der hohen Belastung in der Bodenschicht von ca. $15 \mathrm{~cm}$ bis zur maximalen Meßtiefe $(40 \mathrm{~cm}$ Bodentiefe) deutlich dichter als der Boden ohne Belastung (Tab. 47, Anhang). Die Zunahme der Lagerungsdichte bei Bodenbelastung deutete auf eine Gefügeveränderung hin. Diese Gefügeveränderung spiegelte sich nicht in den EDW wider. 


\subsubsection{Transportfunktionen}

Neben der Verankerung der Pflanze im Boden ist die Wasser- und Nährstoffaufnahme eine der wichtigsten Aufgaben der Wurzel. Alle Prozesse der Stoffaufnahme und -abgabe sind in hohem Maße abhängig von den Eigenschaften, die den umgebenden Boden charakterisieren. Ist der Porenraum eingeschränkt oder sind existierende Poren isoliert und bilden kein vernetztes System, kann die Gasdiffusion im Boden beeinträchtigt sein. Dies kann dazu führen, daß das Entweichen des von den Wurzeln produzierten Kohlendioxids über das Porensystem ebenso behindert wird wie der Eintritt von Sauerstoff in den Boden. Nach einer Literaturstudie von GRABLE \& SIEMER (1968) liegt der für das Pflanzenwachstum kritische Ds/Do-Wert bei 0,02. Fallen die Ds/DoWerte unter 0,02, kann mit Wachstumsstörungen der Pflanzen gerechnet werden. In den Graphiken zum Gasaustausch ist der Ds/Do-Wert 0,02 durch die horizontalen gestrichelten Hilfslinien markiert. Die vertikalen gestrichelten Hilfslinien kennzeichnen ein luftgefülltes Porenvolumen von $0,1 \mathrm{~cm}^{3} / \mathrm{cm}^{3}$. Das entspricht einem Anteil von $10 \%$ am Gesamtporenvolumen.

Für die Wasserbewegung im teilgesättigten Boden stellt die ungesättigte hydraulische Leitfähigkeit ( $\mathrm{ku}$ ) eine Kenngröße dar, unter der die Eigenschaften des Bodens zusammengefaßt werden, welche die Fließbewegung des Wassers als Massenfluß beeinflussen. Solche Einflüsse gehen von der Porengröße und der Porenform (Porengeometrie) aus, die ihrerseits von der Textur und der Struktur des Bodens bestimmt werden.

Die große Bedeutung des Porendurchmessers für die Wasserleitung als Massenfluß drückt sich im starken Abfall von ku mit zunehmender Wasserspannung aus. Ein hoher Anteil an groben Poren in einer Bodenschicht bedingt einen hohen Fließquerschnitt und ermöglicht so eine große Transportrate, solange die Proben mit Wasser gefüllt sind.

\subsubsection{Gasdiffusion}

Auf dem Standort Reinshof wurde im ersten Versuchsjahr bei LBW mit steigender mechanischer Belastung der luftgefüllte Porenraum bei einer Wasserspannung von pF 2,0 in der Bodenschicht von 28-34 cm reduziert (Abb. 10 oben). Diese Reduktion des Porenvolumens (Poren $>30 \mu \mathrm{m}$ Äquivalenzdurch- 
messer) führte zu einer Abnahme des relativen scheinbaren Diffusionskoeffizienten, Ds/Do von ca. 0,05 auf 0,015. Der Kennwert Ds/Do beschreibt das Verhältnis des scheinbaren Diffusionskoeffizienten im Boden (Ds) zum SauerstoffDiffusionskoeffizienten in Luft (Do) und wird im weiteren Verlauf des Textes als Diffusionskoeffizient abgekürzt.

LBW

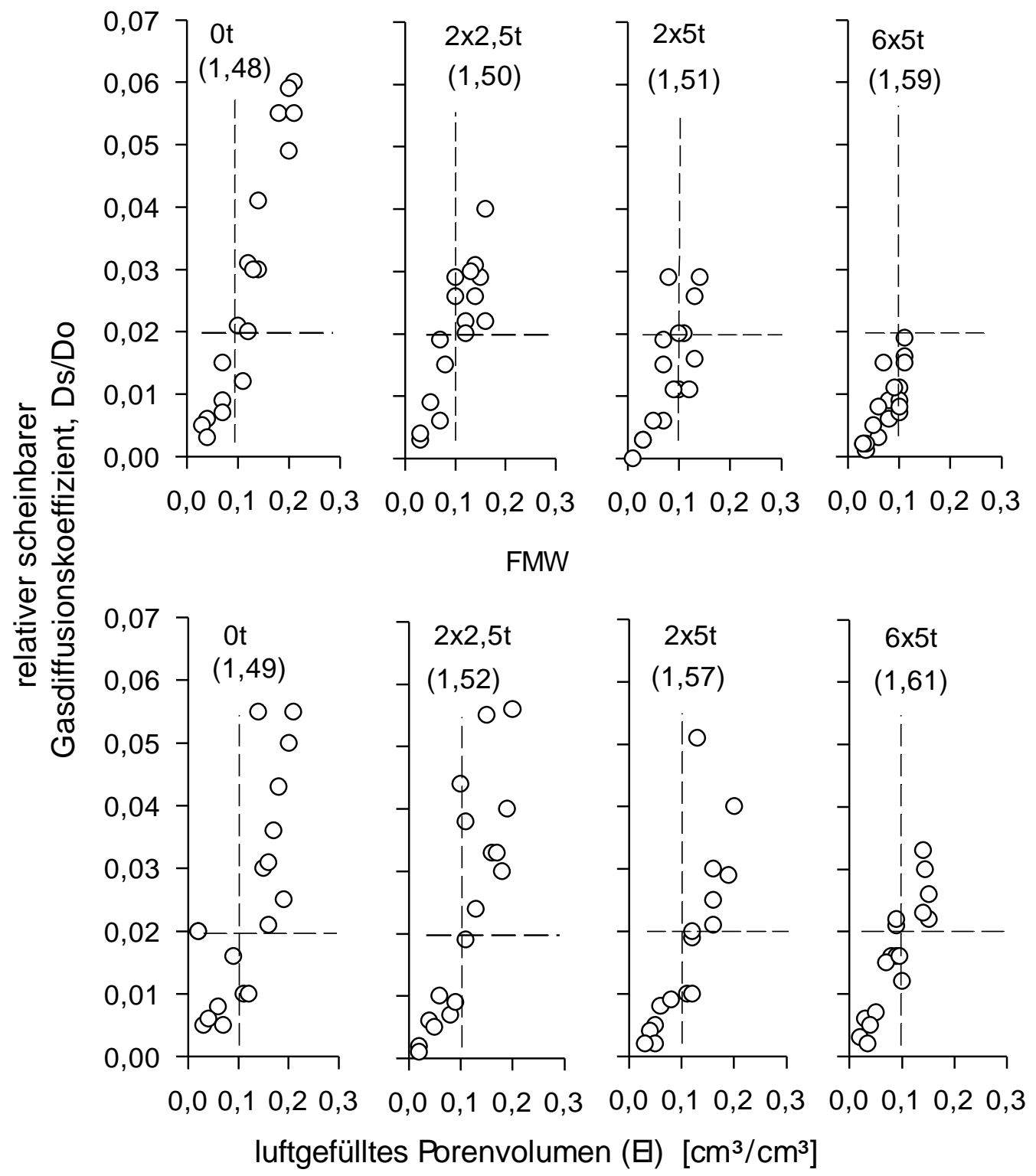

Abb. 10: Beziehung zwischen Ds/Do und dem luftgefüllten Porenvolumen (El) auf den Standort Reinshof am12.04.1995 in 28-34 cm Bodentiefe. Die Zahlen in Klammern geben Mittelwerte der Lagerungsdichte des Bodens $\left[\mathrm{g} / \mathrm{cm}^{3}\right]$ in der untersuchten Probe an. Die gestrichelten Hilfslinien werden im Text erklärt. Die Ds/Do-Werte bei pF 2,0 sind durch ausgefüllte Symbole dargestellt. 
Besonders deutlich zeigte sich die Begrenzung des Diffusionskoeffizienten auf hoch belastetem Boden. Bei dieser Variante lagen alle Diffusionskoeffizienten unter 0,02.

Auf dem Boden mit FMW stellte sich die Abnahme des luftührenden Porenvolumens und der Diffusionskoeffizienten bei mechanischer Belastung weniger drastisch dar als auf gepflügtem Boden (Abb. 10 unten): Bei geringer Belastung zeigte sich keine Veränderung zur Kontrolle. Erst bei mittlerer Belastung nahmen der luftgefüllte Porenraum und der Diffusionskoeffizient ab. Diese Tendenz setzte sich bei hoher Belastung fort. Die Diffusionskoeffizienten sanken noch weiter ab, erreichten aber nicht die Werte von LBW 6x5t. Mit der Abnahme des luftührenden Porenraumes (EI) und der Diffusionskoeffizienten ging die Zunahme der Lagerungsdichte einher (Abb. 10).

Auf dem Standort Relliehausen (1995) führte die mittlere und hohe mechanischen Bodenbelastung bei LBW zu einer starken Zunahme der Lagerungsdichte, verbunden mit einer Reduktion des luftührenden Porenraumes. Dies zog eine drastischen Beeinträchtigung der Diffusion nach sich (Abb. 11). Im Gegensatz zu Reinshof (Abb. 10 oben) war in Relliehausen auf dem gepflügten Boden - vor allem bei mittlerer und hoher Bodenbelastung - die Abnahme der Diffusionskoeffizienten stärker. Bei LBW 2x5t lagen die Ds/Do- Wert bis auf eine Ausnahme unter 0,02. Bei LBW 6x5t wurde der Ds/Do- Wert 0,02 deutlich unterschritten. Bei FMW nahm die Lagerungsdichte in Relliehausen mit steigender Belastung deutlich zu (Abb. 11 unten). Wie auch bei gepflügtem Boden wurde bei einer höheren Dichte des Bodens eine Reduktion des luftgefüllten Porenraumes und eine Beeinträchtigung der Diffusion (Abb. 11) gemessen, aber doch weit weniger ausgeprägt als bei LBW. 
LBW

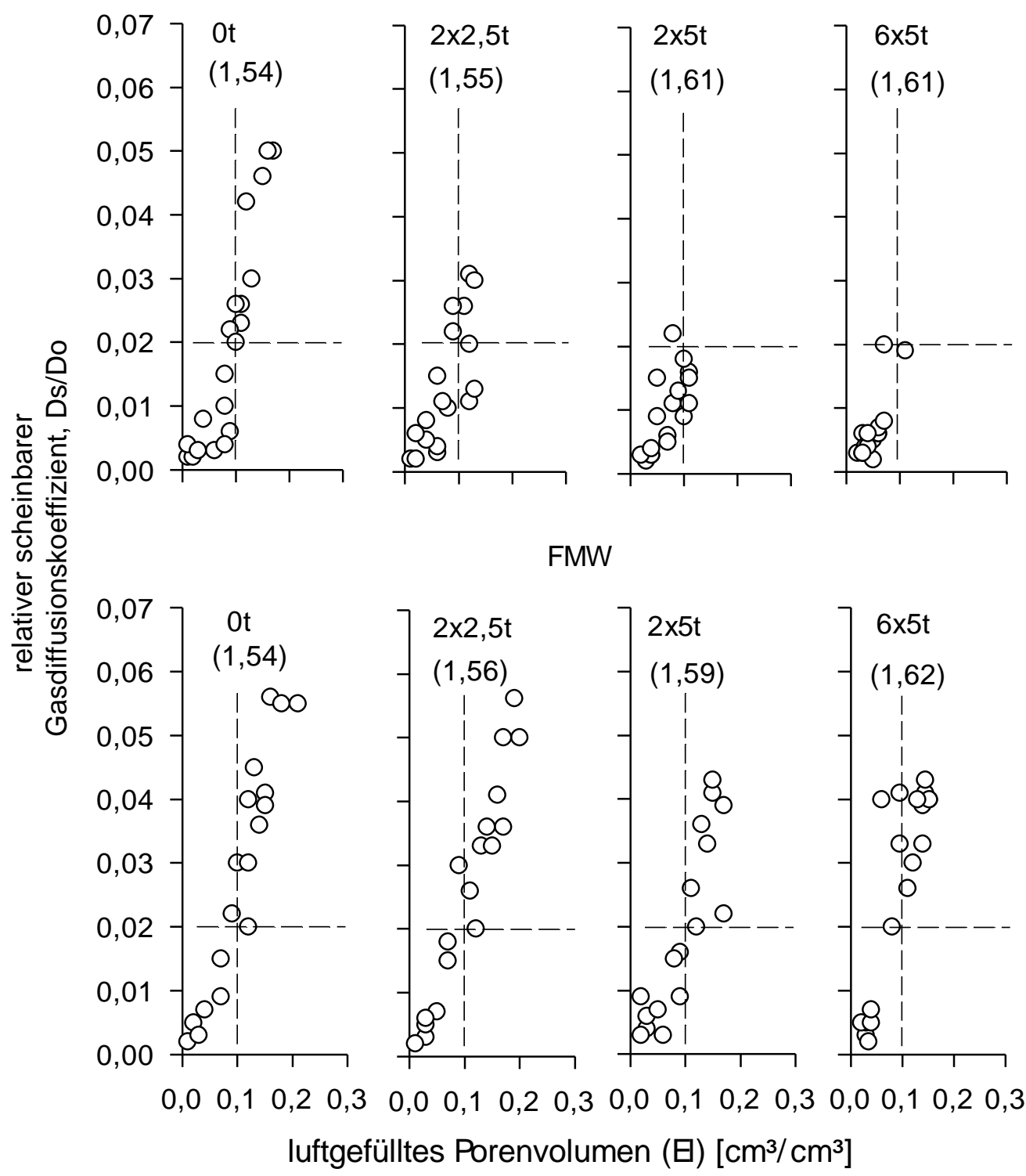

Abb. 11: Beziehung zwischen Ds/Do und dem luftgefüllten Porenvolumen (El) auf den Standort Relliehausen am 26.04.1995 in 28-34 cm Bodentiefe. Die Zahlen in Klammern geben Mittelwerte der Lagerungsdichte des Bodens $\left[\mathrm{g} / \mathrm{cm}^{3}\right]$ in der untersuchten Probe an. Die gestrichelten Hilfslinien werden im Text erklärt. Die Ds/Do-Werte bei pF 2,0 sind durch ausgefüllte Symbole dargestellt.

Wie in Reinshof und Relliehausen kam es in Großobringen auf gepflügtem Boden bei mechanischer Bodenbelastung zu einem Anstieg der Lagerungsdichte, verbunden mit einem Verlust an luftgefülltem Porenraum und einer Beeinträchtigung der Diffusion (Abb.12). 
LBW tief

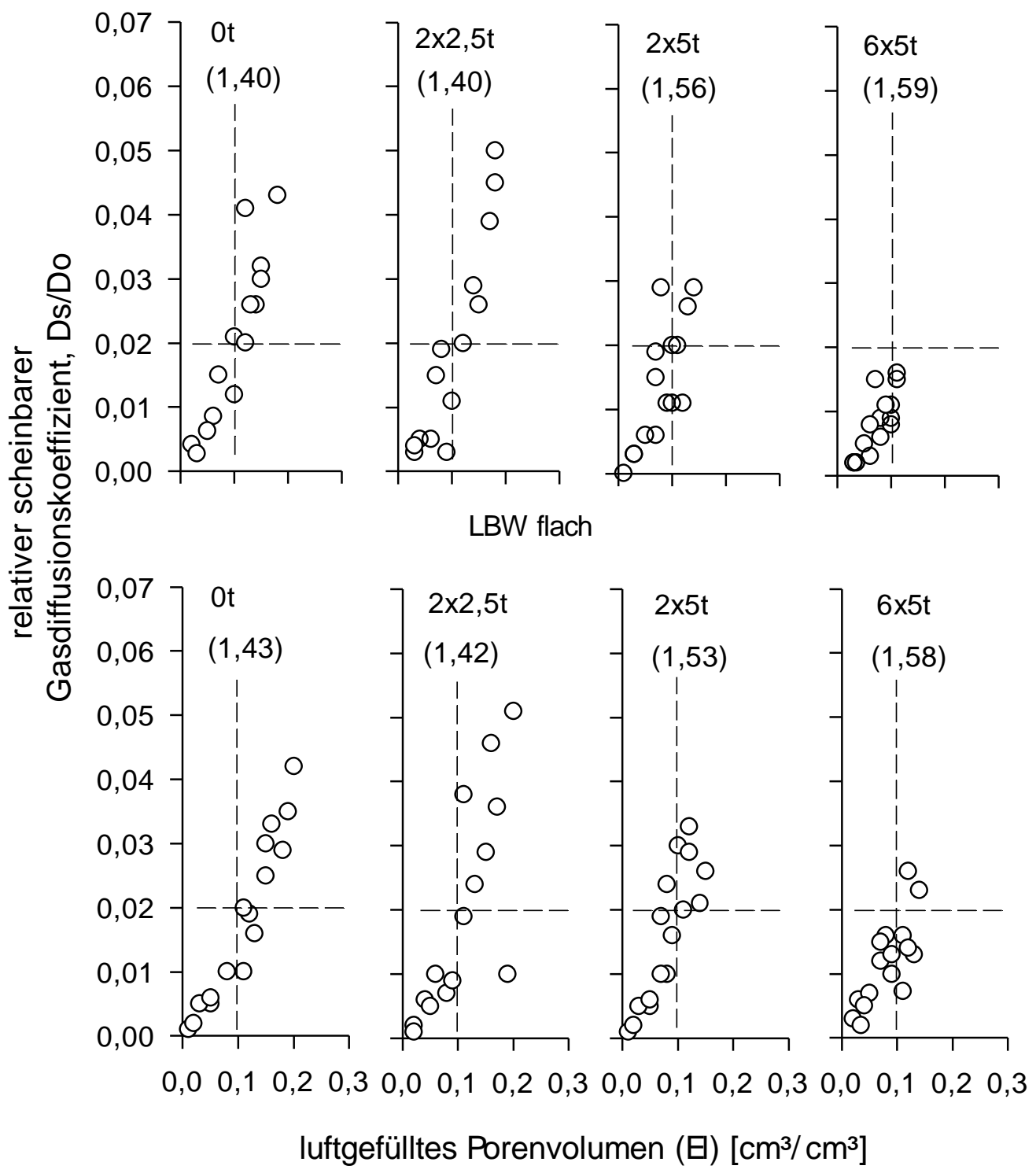

Abb. 12: Beziehung zwischen Ds/Do und dem luftgefüllten Porenvolumen (El) auf den Standort Großobringen am 24.03.1995 in 28-34 cm Bodentiefe. Die Zahlen in Klammern geben Mittelwerte der Lagerungsdichte des Bodens $\left[\mathrm{g} / \mathrm{cm}^{3}\right]$ in der untersuchten Probe an. Die gestrichelten Hilfslinien werden im Text erklärt. Die Ds/Do-Werte bei pF 2,0 sind durch ausgefüllte Symbole dargestellt.

Bei beiden Bearbeitungssystemen führte die geringe Bodenbelastung nicht zu einem Anstieg der Lagerungsdichte oder zu einer Beeinträchtigung der Diffusion. Erst bei mittlerer und hoher Bodenbelastung wurden in beiden Systemen die Lagerungsdichten erhöht und die Diffusionskoeffizienten reduziert. Dies zeigte sich bei tiefer Bodenbearbeitung deutlicher als bei flacher Bearbeitung. 
Auf dem Standort Reinshof wurden im Frühjahr 1997 erneut Messungen der Gasdiffusion durchgeführt (Abb. 13). Die Untersuchung sollte zeigen, ob sich die Bedingungen vor allem auf hoch belastetem Boden bei LBW über die Zeit geändert haben.

LBW

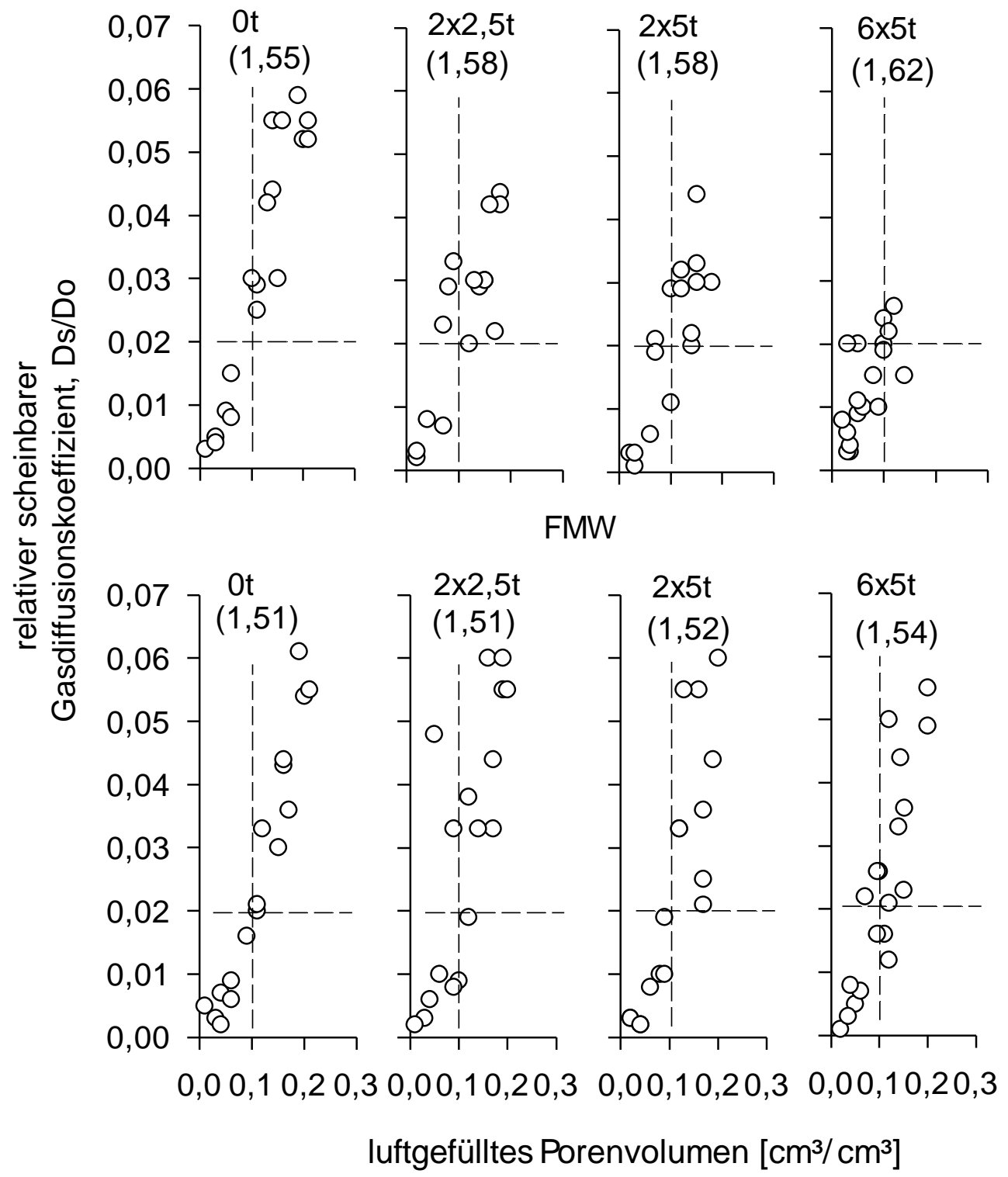

Abb. 13: Beziehung zwischen Ds/Do und dem luftgefüllten Porenvolumen (EI) auf den Standort Reinshof am16.04.1997 in 28-34 cm Bodentiefe. Die Zahlen in Klammern geben Mittelwerte der Lagerungsdichte des Bodens $\left[\mathrm{g} / \mathrm{cm}^{3}\right]$ in der untersuchten Probe an. Die gestrichelten Hilfslinien werden im Text erklärt. Die Ds/Do-Werte bei pF 2,0 sind durch ausgefüllte Symbole dargestellt. 
Bei LBW konnte auch zwei Jahre nach der mechanischen Belastung eine Abnahme des luftgefüllten Porenraumes, gekoppelt mit einer Abnahme der Diffusionskoeffizienten bei geringer, mittlerer und hoher Belastung gemessen werden (Abb. 13 oben). Dennoch sind Veränderungen aufgetreten: Die Abnahme der Diffusionskoeffizienten von LBW 0t nach 2×2,5t und 2x5t war 1997 auf dem gepflügten Boden (Abb. 13 oben) deutlich geringer als 1995 (Abb. 10 oben). Bei einem Vergleich des ersten und dritten Versuchsjahres der Variante LBW 6x5t deutete sich die Zunahme des Porenanteils > 30 um Äquivalenzdurchmesser an (Abb. 13 oben, ausgefüllte Symbole). Der größere Porenanteil führte zu einem Anstieg der Diffusionskoeffizienten, der Ds/Do- Wert von 0,02 wurde im Gegensatz zu 1995 überschritten.

Wie im ersten Versuchsjahr waren 1997 die Diffusionskoeffizienten als Funktion des luftgefüllten Porenvolumens auf dem flach bearbeiteten und gemulchten Boden bei keiner, geringer und mittlerer Bodenbelastung annähernd identisch (Abb. 13 unten). Insgesamt lagen bei diesen Varianten 1997 die Diffusionskoeffizienten geringfügig über denen von 1995. Auf dem hoch belasteten Boden zeigte sich 1997, im Vergleich zu 1995, ein deutlicher Anstieg der Diffusionskoeffizienten.

Die Lagerungsdichten des gepflügten Bodens lagen 1997 (Abb. 13 oben) bei keiner, geringer und mittlerer Bodenbelastung auf gleichem Niveau, insgesamt aber auffallend höher als im ersten Versuchsjahr (Abb. 10 oben). Bei hoher Belastung wurde im Vergleich zu den ersten drei Belastungsstufen und dem Jahr 1995 die höchste Lagerungsdichte gemessen. Auf dem flach bearbeiteten und gemulchten Boden stieg die Lagerungsdichte von $1,44 \mathrm{~g} / \mathrm{cm}^{3}$ auf dem Boden ohne Belastung auf $1,58 \mathrm{~g} / \mathrm{cm}^{3}$ bei hoher Bodenbelastung an (Abb. 13 unten). Diese Werte entsprachen in etwa den Lagerungsdichten des Jahres 1995 (Abb. 10 unten).

\subsubsection{Ungesättigte Wasserleitfähigkeit}

Auf dem Standort Reinshof verlief die Funktion der ungesättigten hydraulischen Leitfähigkeit in der Bodentiefe $28-34 \mathrm{~cm}$ bei LBW 0t, 2x2,5t und 2x5t sowie bei FMW (alle Belastungsstufen) nahezu auf gleicher Höhe (Abb. 14). 
Durch die Belastung mit 6x5t wurde die ungesättigte hydraulische Leitfähigkeit bei einer Wasserspannung zwischen 10 und 50 hPa auf dem gepflügten Boden stark reduziert.

LBW

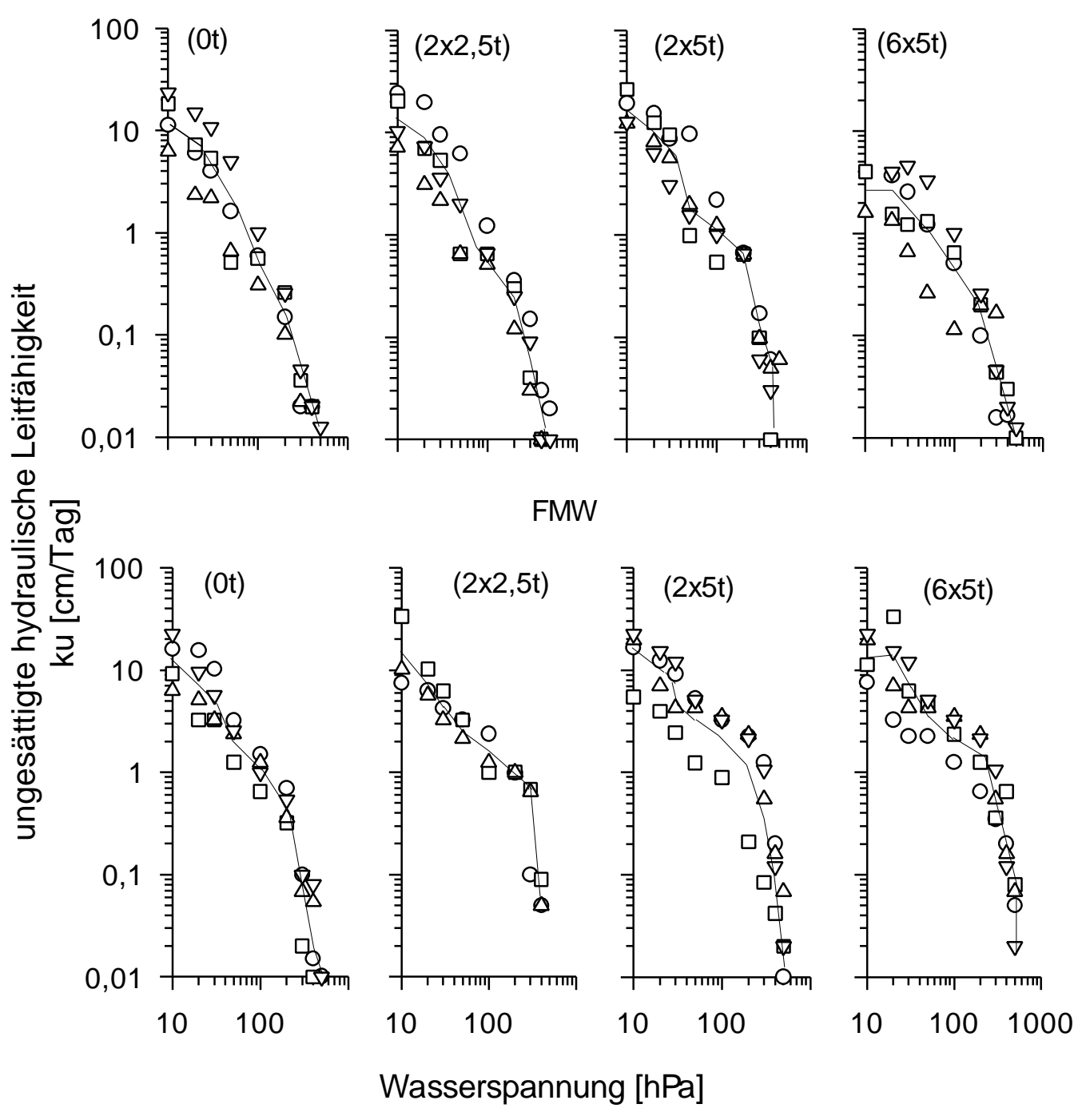

Abb. 14: Beziehung zwischen der ungesättigten hydraulischen Leitfähigkeit (ku) und der Wasserspannung des Bodens in der Bodentiefe $28-34 \mathrm{~cm}$ am 12.04.1995 auf dem Standort Reinshof. Gleiche Symbole stehen für die Meßwerte gleicher Stechzylinderproben. Die durchgezogene Linie verbindet die geometrischen Mittel der ku- Werte bei gleicher Wasserspannung miteinander.

Auf dem Standort Relliehausen stellte sich die ungesättigte hydraulische Leitfähigkeit in der Bodentiefe 28-34 cm ähnlich dar wie auf dem Standort Reinshof: Nur bei LBW 6x5t und einer Wasserspannung zwischen 10 und $50 \mathrm{hPa}$ lag die ungesättigte hydraulische Leitfähigkeit deutlich unter den Werten der 
drei anderen Belastungsstufen (LBW und FMW) bei gleicher Wasserspannung (Abb. 15).

LBW

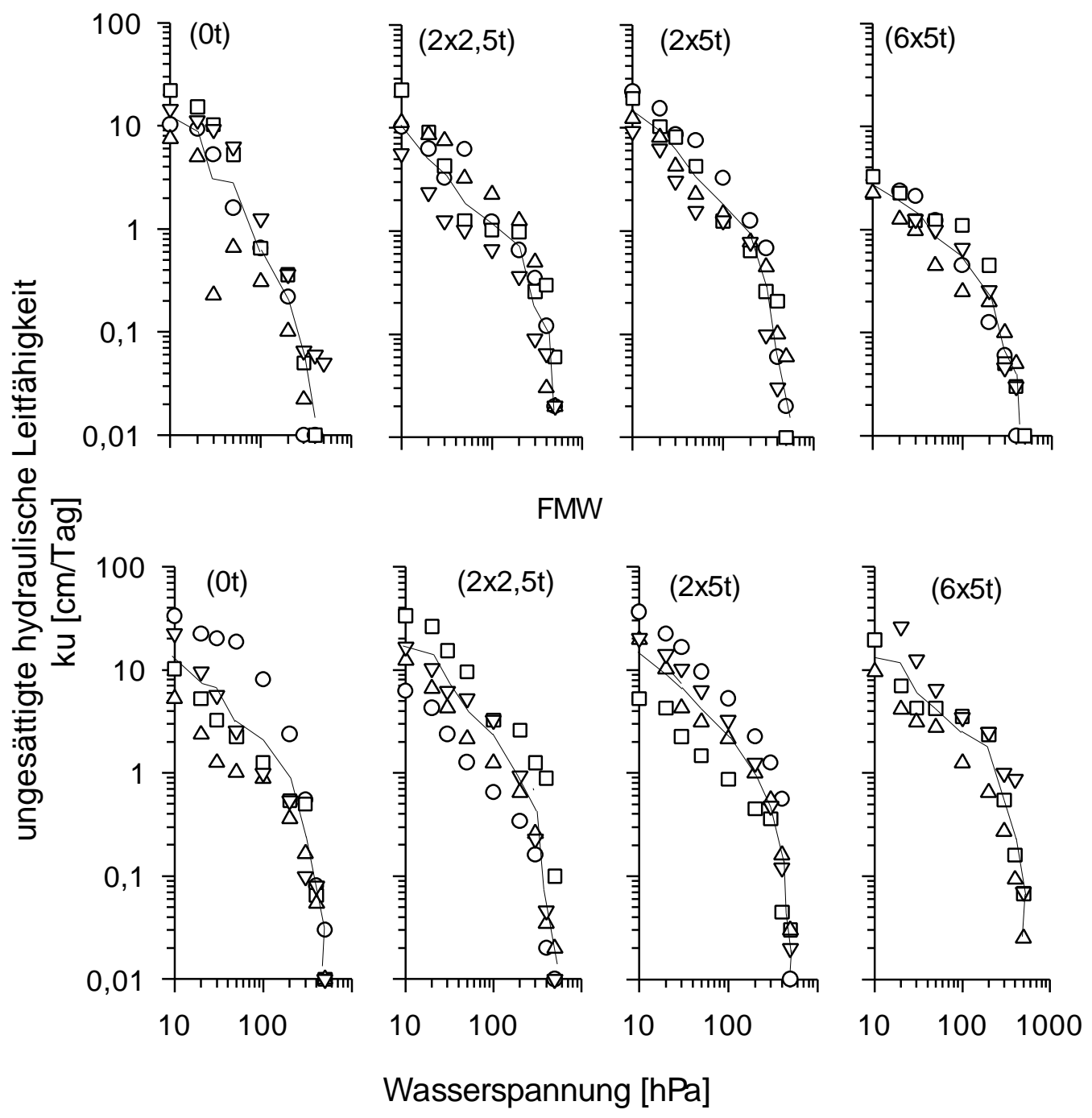

Abb. 15: Beziehung zwischen der ungesättigten hydraulischen Leitfähigkeit (ku) und der Wasserspannung des Bodens in der Bodentiefe $28-34 \mathrm{~cm}$ am 26.04.1995 auf dem Standort Relliehausen. Gleiche Symbole stehen für die Meßwerte gleicher Stechzylinderproben. Die durchgezogene Linie verbindet die geometrischen Mittel der ku- Werte bei gleicher Wasserspannung miteinander.

Auf dem Standort Großobringen fiel die ungesättigte hydraulische Leitfähigkeit in der Bodenschicht 28-34 cm bei LBW tief und LBW flach annähernd identisch aus (Abb. 16). Die Werte auf dem tief und flach bearbeiteten Boden la- 
gen bei hoher Bodenbelastung und einer Wasserspannung von ca. 10-50 hPa deutlich unter den Werten der anderen Belastungsstufen.

LBW tief

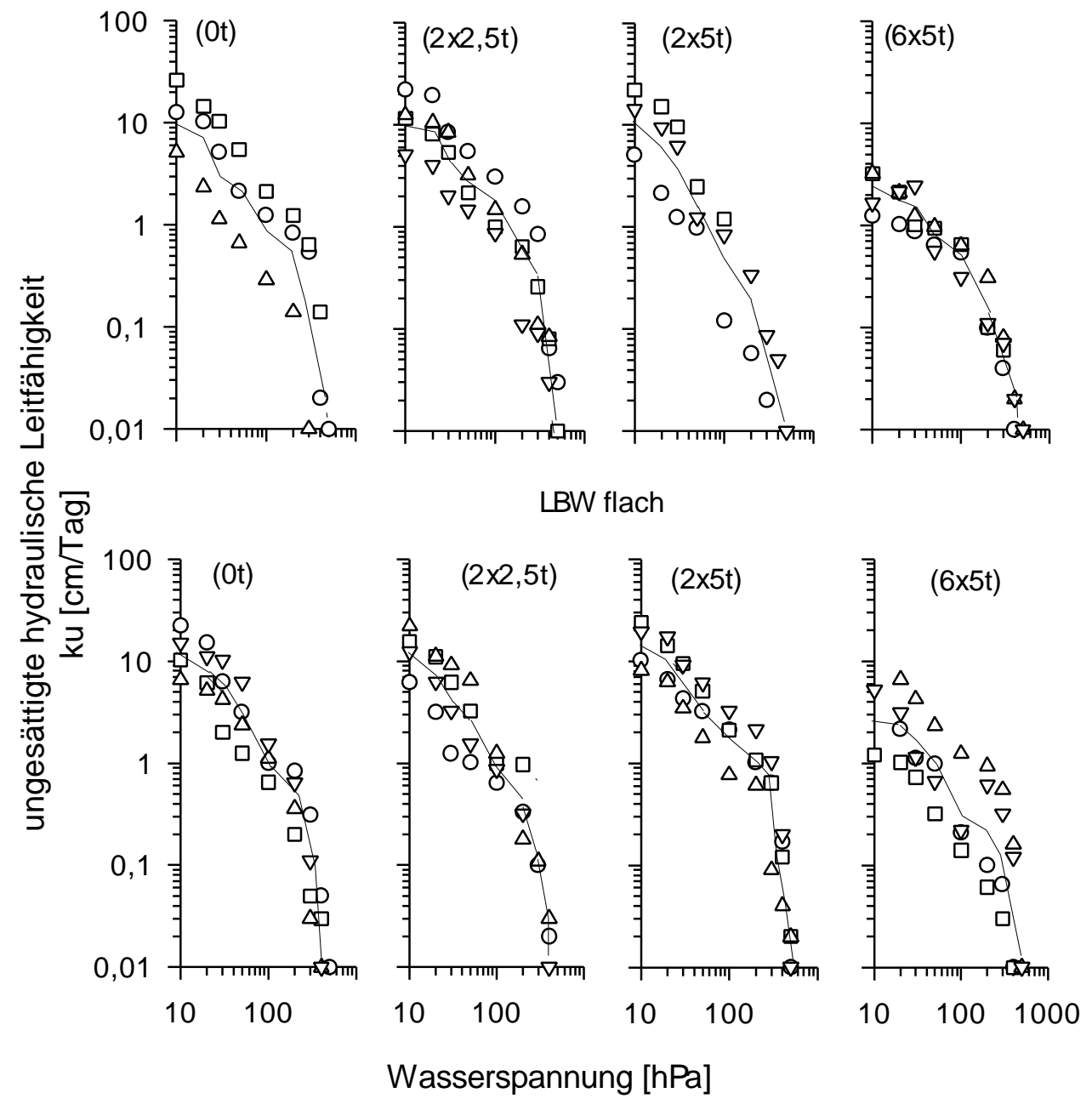

Abb. 16: Beziehung zwischen der ungesättigten hydraulischen Leitfähigkeit (ku) und der Wasserspannung des Bodens in der Bodentiefe $28-34 \mathrm{~cm}$ am 24.03.1995 auf dem Standort Großobringen. Gleiche Symbole stehen für die Meßwerte gleicher Stechzylinderproben. Die durchgezogene Linie verbindet die geometrischen Mittel der ku-Werte bei gleicher Wasserspannung miteinander.

In Großobringen war der Unterschied zwischen den ersten drei Belastungsstufen zur hoch belasteten Stufe in den ku-Werten bei niedriger Wasserspannung größer (eine Zehnerpotenz) als bei den anderen Standorten. Zudem wurde im Vergleich zu den Standorten Reinshof (LBW) und Relliehausen (LBW) in Großobringen die ungesättigte hydraulische Leitfähigkeit bei einer Wasserspannung zwischen 10 und $50 \mathrm{hPa}$ durch den hohen Lasteintrag auf beiden Bearbeitungsvarianten stärker reduziert. 


\subsection{Bestandesentwicklung}

Im vorangegangenen Kapitel wurde aufgezeigt, welche Auswirkung die mechanische Bodenbelastung auf den EDW, die Lagerungsdichte und die Transportfunktionen der Böden für Gas und Wasser hatte. Die Untersuchung der Wurzelsysteme sollte zeigen, wie die Pflanzen auf die auflastbedingte Veränderung der Wachstumsbedingungen im Boden reagieren. Neben dem Wasser- und Nährstoffangebot des Bodens und seiner Leitfähigkeit für Wasser und Gas hat die Größe und die Leistungsfähigkeit des Wurzelsystems einen direkten Einfluß auf das oberirdische Pflanzenwachstum. Um Auswirkungen der mechanischen Bodenbelastung über das Wurzelsystem auf Sproß und Blattwachstum zu erfassen, wurde die Massezunahme der oberirdischen Pflanzenteile und die Ausprägung der Blattfläche festgehalten. Messungen der Gesamtwasserspannung und des $\mathrm{CO}_{2} / \mathrm{H}_{2} \mathrm{O}$-Gaswechsels der Pflanzen sollten zeigen, inwieweit die mechanische Bodenbelastung - über Wurzel- und Sproßwachstum - eine Einschränkung der Photosyntheseleistung bewirken kann.

\subsubsection{Wurzelsystem}

\subsubsection{Standort Reinshof}

Für den Standort Reinshof wird das Wurzelsystem der Sommergerste (1995) und der Wintergerste (1997) zunächst an Wurzelprofilen in den Abb. 17 und 18 vorgestellt. Auf die Darstellung der Wurzelprofile des Winterweizes (1996) wird an dieser Stelle verzichtet, da das Wurzelwachstum des Weizens nur schwach durch die Bodenbelastung beeinträchtigt wurde. Eine Darstellung der WLD des Winterweizens 1996 findet sich in den Tab. 52-54 im Anhang. Im Anschluß an die Beschreibung der Wurzelprofile soll die GWL und die maximale Durchwurzelungstiefe in Abb. 19 betrachtet werden. Am Wurzelprofil lassen sich unschwer Bearbeitungshorizonte, durchwurzelte Bioporen und Durchwurzelungswiderstände erkennen: Horizontale Übergänge von hoher zu geringerer Punktdichte trennen bei LBW in ca. $25 \mathrm{~cm}$ Bodentiefe den bearbeiteten, aufgelokkerten Boden vom unbearbeiteten Unterboden (Beispiel: gestrichelte Hilfslinie in Abb. 18 LBW 2x5t). Bei dem nicht gepflügten Boden wurden bei der Aussaat durch die vorlaufende Kreiselegge die ersten 5 bis $7 \mathrm{~cm}$ des Bodens aufgelok- 
kert. Hohe Punktdichten in dieser Schicht spiegeln eine hohe Durchwurzelungsdichte wider. Wird eine Biopore intensiv durchwurzelt, zeigt das Wurzelprofil eine vertikal ausgerichtete, eng und scharf begrenzte Fläche mit deutlich erhöhter Punktdichte (Beispiel: gestrichelte Hilfslinie in Abb. 18 FMW 2x5t).

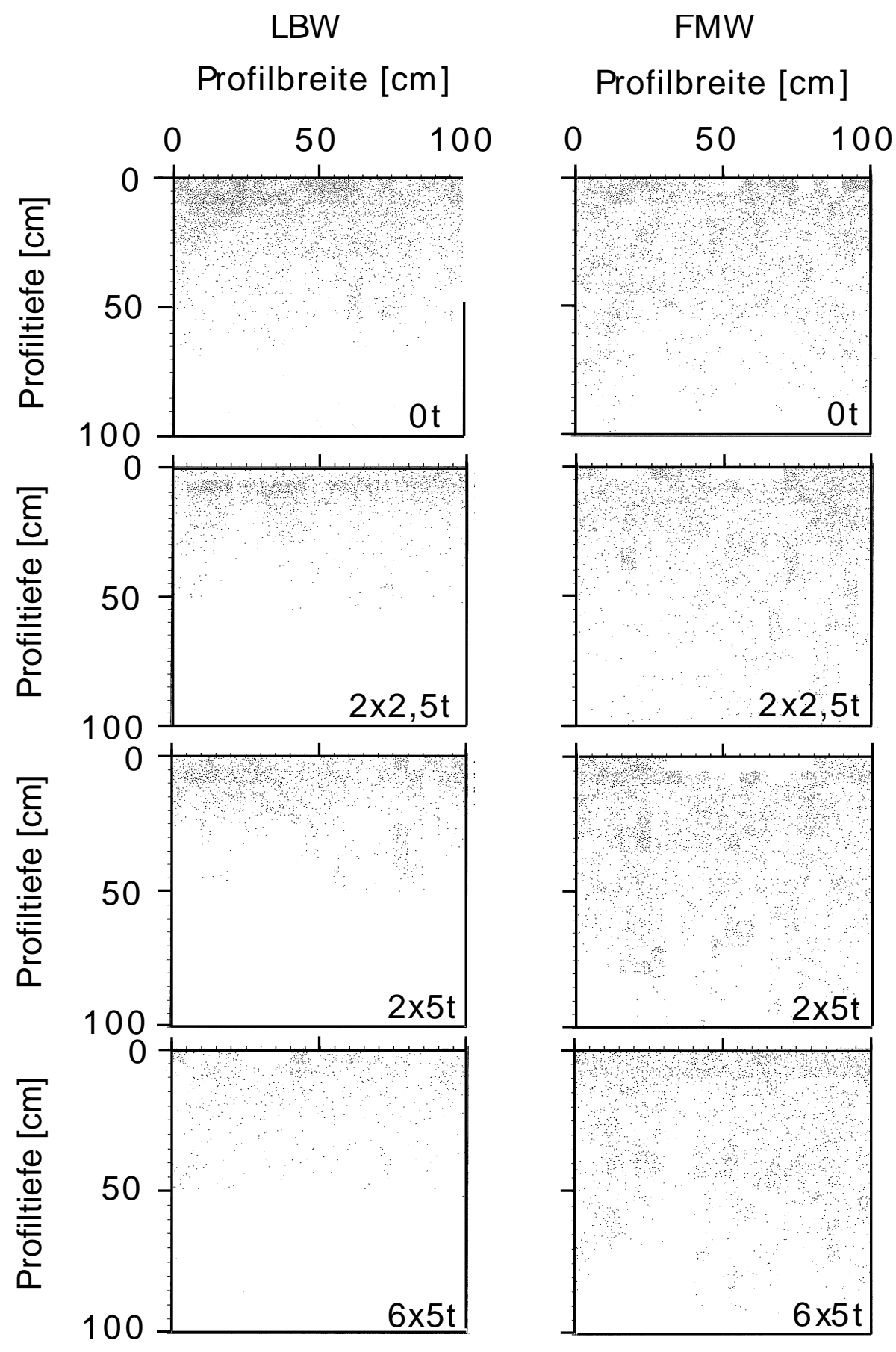

Abb.17: Wurzelprofil für Sommergerste nach der Profilwandmethode im Versuchsjahr 1995 auf dem Standort Reinshof. Die Zählung wurde zum Stadium EC 45 (03.07.1995) durchgeführt. Jeder Punkt entspricht einer Wurzellängeneinheit von $0,5 \mathrm{~cm}$. 

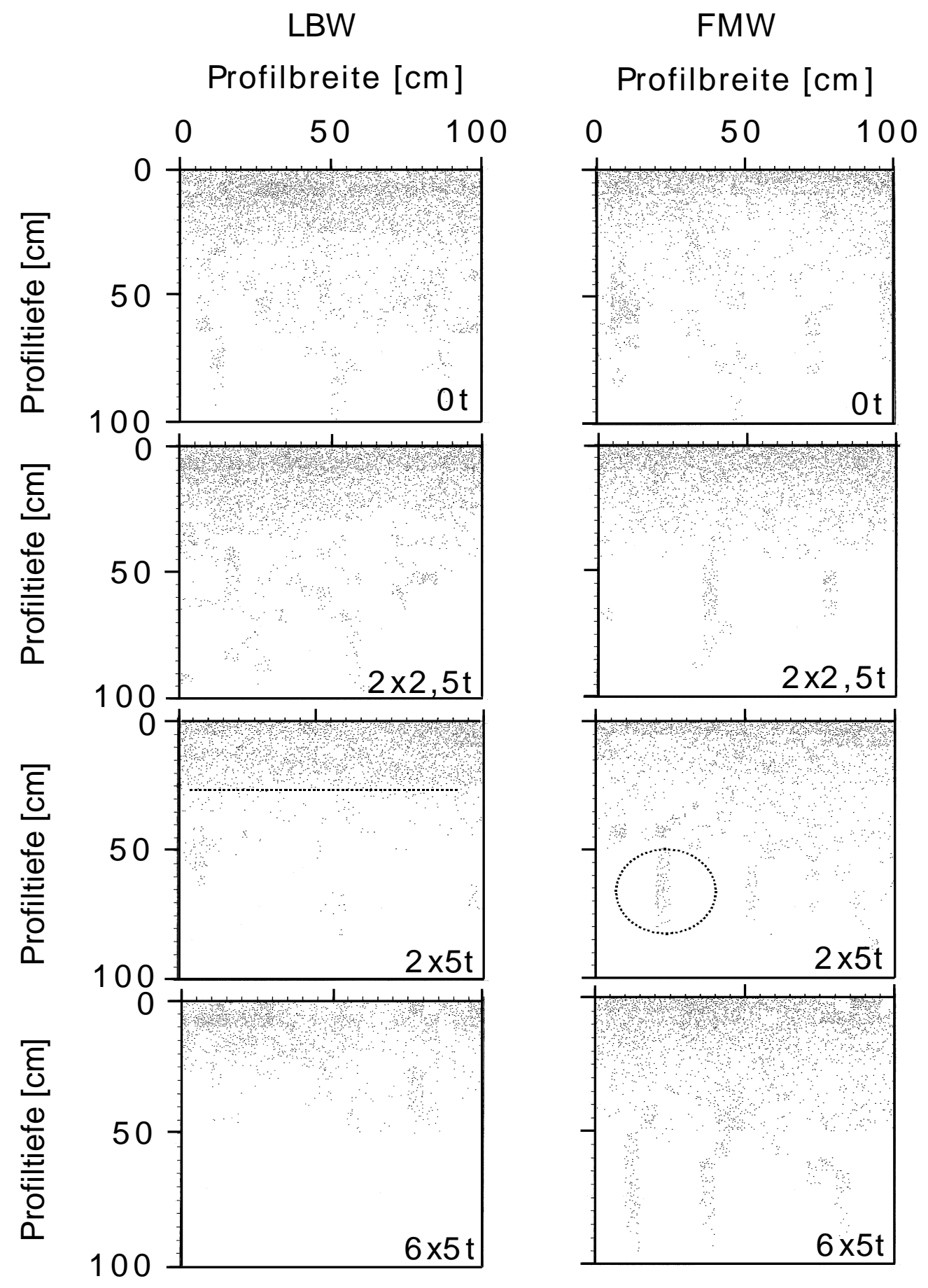

Abb. 18: Wurzelprofil für Wintergerste nach der Profilwandmethode im Versuchsjahr 1997 auf dem Standort Reinshof. Die Zählung wurde zum Stadium EC 45 (02.06.1997) durchgeführt. Jeder Punkt entspricht einer Wurzellängeneinheit von $0,5 \mathrm{~cm}$. Die gestrichelten Hilfslinien werden im Text erläutert. 
Im ersten Versuchsjahr war das Wurzelsystem der Sommergerste auf dem gepflügten Boden mit geringer, mittlerer und hoher Bodenbelastung gegenüber dem nicht belasteten Boden deutlich eingeschränkt: Über die gesamte Durchwurzelungstiefe nahm die Wurzellängendichte mit steigender Belastung des Bodens ab. Der Grad der Einschränkung war bei hoher Belastung stärker als bei geringer und mittlerer Bodenbelastung.

Auf dem flach bearbeiteten Boden (Abb. 17 rechts) konnten 1995 keine Auswirkungen der Bodenbelastung auf die WLD festgestellt werden. Ein Vergleich der Bearbeitungssysteme zeigte für FMW eine Durchwurzelung mit höherer Intensität und größerer Tiefe.

Im Jahr 1997 - zwei Jahre nach der mechanischen Bodenbelastung - war die Wurzellängendichte der Wintergerste von LBW 0t und LBW 2x2,5t auf gleichem Niveau (Abb. 18 links). Deutlich geringer stellte sie sich bei LBW 2x5t und 6x5t dar. Auf dem gepflügten Boden ist bei allen Belastungsstufen die Pflugsohle oder Krumenbasisverdichtung deutlich an der sprunghaften Abnahme der Punktdichte in ca. $25 \mathrm{~cm}$ Tiefe zu erkennen (Beispiel: gestrichelte Hilfslinie in Abb. 18 LBW 2x5t). Das Wurzelprofil von 2x5t und 6x5t zeigt, wie stark eine Pflugsohle als Durchwurzelungswiderstand wirken kann: Nur vereinzelt konnten Wurzeln den verdichteten Horizont durchbrechen. Eine Durchwurzelung des Unterbodens fand kaum statt. Bei FMW konnte im letzten Versuchsjahr (Abb. 18 rechts) kein Einfluß der mechanischen Bodenbelastung auf das Wurzelwachstum festgestellt werden.

Aus der WLD der Bodenschichten errechnet sich die GWL. Sie wird angegeben in km Wurzellänge unter $1 \mathrm{~m}^{2}$ Bodenoberfläche. Abb. 19 zeigt die GWL bei den Bearbeitungssystemen LBW und FMW auf dem Standort Reinshof in den drei Versuchsjahren. Die Darstellung der GWL - unterteilt in Ober- und Unterboden - soll einen Vergleich der Wurzelsysteme beider Bearbeitungssysteme und aller Belastungsstufen vereinfachen. 


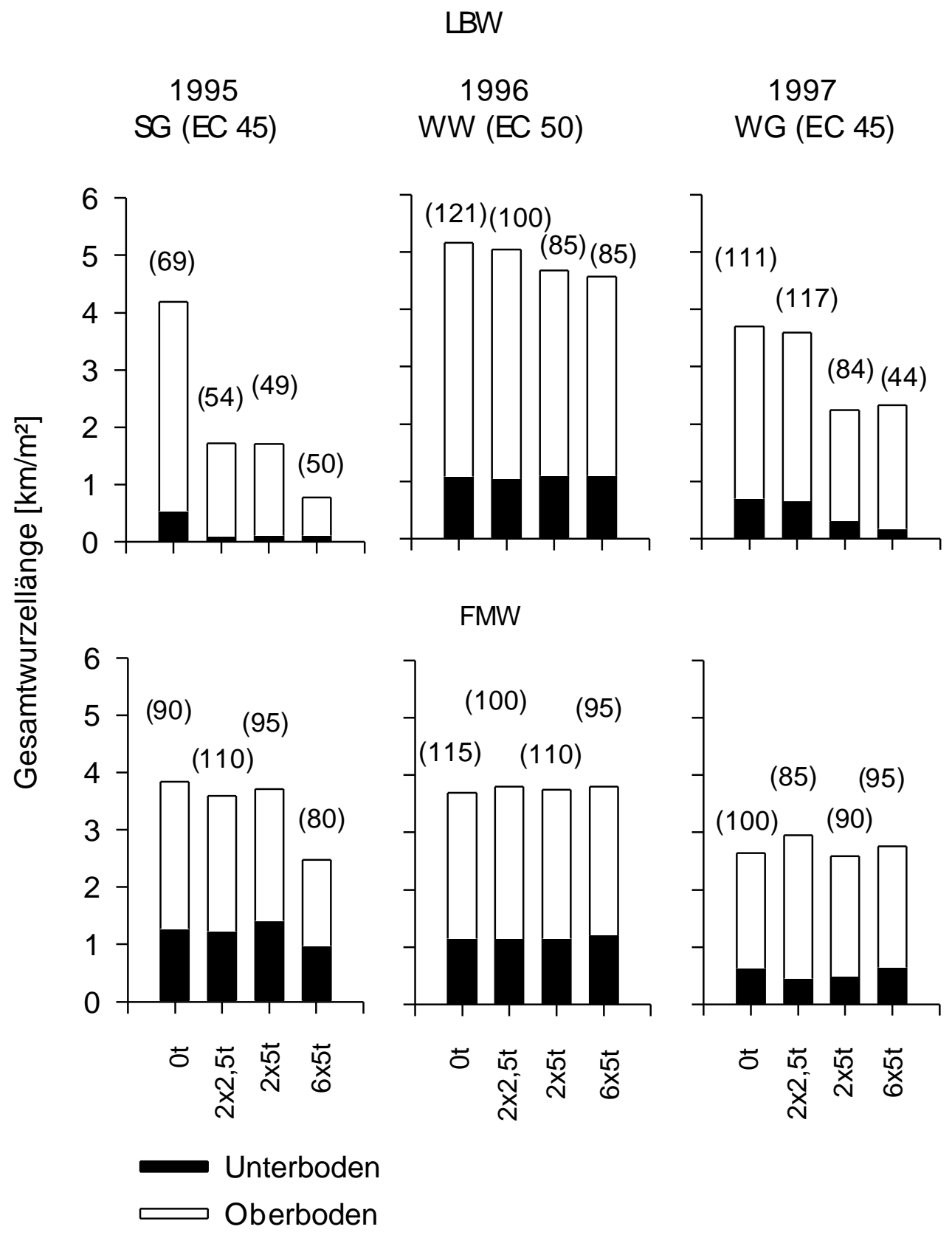

Abb.19: Gesamtwurzellänge (GWL) und GWL in Oberboden (0-30 cm Bodentiefe, weiße Säulen) und Unterboden (von $30 \mathrm{~cm}$ Bodentiefe - maximale Durchwurzelungstiefe, schwarze Säulen) auf dem Standort Reinshof. Dargestellt sind auch die Beprobungstermine und die angebauten Feldfrüchte: 03.07.1995 Sommergerste (SG); 12.06.1996 Winterweizen (WW); 02.06.1997 Wintergerste (WG). Die Zahlen in Klammern zeigen die maximale Durchwurzelungstiefe $[\mathrm{cm}]$. 
Bei LBW 1995 (Abb. 19 oben links) ist die Auswirkung der mechanischen Bodenbelastung auf das Wurzelsystem der Sommergerste offensichtlich: Verglichen mit dem unbelasteten Boden führte die geringe und mittlere Belastung zu einer Reduktion der GWL um 59 \%. Die GWL auf dem hoch belasteten Boden erreichte nur $18 \%$ der GWL auf der Kontrolle (LBW 0t). Besonders drastisch war der Effekt der Belastung auf die Durchwurzelung des Unterboden: Die GWL des Unterbodens bei geringer, mittlerer und hoher Bodenbelastung erreichte nur ca. $15 \%$ des entsprechenden Wertes im unbelasteten Boden. Neben der Gesamtlänge des Wurzelsystems wurde auch die Tiefe der Durchwurzelung beeinträchtigt. Die Sommergerste auf dem Boden ohne Belastung (LBW 0t) wurzelte etwa $18 \mathrm{~cm}$ tiefer als bei geringer, mittlerer und hoher Bodenbelastung. Im Versuchsjahr 1996 (Abb. 19 oben mitte) zeigte das Wurzelsystem des Winterweizens - verglichen mit der Sommergerste - im Ober- und Unterboden eine geringere Empfindlichkeit gegen die mechanische Bodenbelastung. Dennoch wurden Unterschiede zwischen den Belastungsstufen gemessen. Auf dem gepflügten Boden nahm die Durchwurzelung des Oberbodens mit steigender Belastung merklich ab. Die GWL fiel von ot nach 6x5t um $12 \%$ ab. Die WLD im Unterboden war bei allen Belastungsstufen annähernd gleich. Bei $2 \times 5 t$ und 6x5t erreichte das Wurzelsystem eine um ca. $19 \%$ geringere Tiefe als bei $\mathbf{0 t}$ und 2x2,5t. Im dritten Versuchsjahr (Abb. 19 oben rechts) lag die GWL der Wintergerste bei $0 t$ und $2 \times 2,5 t$ auf gleichem Niveau. Bei $2 \times 5 \mathrm{t}$ und $6 \times 5 \mathrm{t}$ nahm die GWL um ca. $36 \%$ ab. Die GWL des Unterbodens erreichte bei 2x5t $43 \%$ und bei $6 \times 5 t$ nur $24 \%$ der Kontrolle (LBW 0t).

Bei FMW (Abb. 19 unten links) zeigte im ersten Versuchsjahr das Wurzelsystem der Sommergerste nur auf dem hoch belasteten Boden eine Reduktion der GWL: Im Oberboden erreichten die Werte $58 \%$, im Unterboden $75 \%$ der Kontrolle (FMW 0t). Nicht nur die WLD, sondern auch die Durchwurzelungstiefe war eingeschränkt. Im zweiten und dritten Versuchsjahr lagen die Werte der GWL im Ober- und Unterboden bei allen Belastungsstufen auf einem Niveau. Auch wurde die maximalen Durchwurzelungstiefe nicht deutlich reduziert (Abb. 19 mitte und rechts). Ein Vergleich der Bearbeitungssysteme zeigte 1995 vor allem bei der Unterbodendurchwurzelung eine größere Streßverträglichkeit der FMW: Bei zunehmender Radlast und steigender Überrollhäufigkeit blieb die Durchwurzelung des Unterbodens bei FMW annähernd gleich. Bei LBW hinge- 
gen führte die mechanische Bodenbelastung zu einer drastischen Abnahme der Durchwurzelung des Unterbodens. Im zweiten Versuchsjahr war bei FMW die Oberbodendurchwurzelung beträchtlich geringer als bei LBW nach Pflugeinsatz. Die Unterbodendurchwurzelung erreichte unabhängig von der Belastungsstufe bei beiden Bearbeitungssystemen dieselbe Höhe. So wie im ersten Versuchsjahr, so konnte auch im dritten Versuchsjahr eine größere Unempfindlichkeit des flach bearbeiteten Bodens gegenüber mechanischer Belastung festgestellt werden: Die Durchwurzelung des Ober- und Unterbodens stellte sich - im Gegensatz zum gepflügten Boden (Abb. 19 oben rechts) - unabhängig von der Belastungsstufe dar (Abb. 19 unten rechts).

Im Rahmen einer Diplomarbeit (MERTEN1998) wurde auf dem Standort Reinshof auf Block 2 im Jahr 1996 der Wurzeldurchmesser des Winterweizens in drei Bodentiefen gemessen (Abb. 20). Die Beprobungstiefen wurden so gewählt, daß bei LBW Messungen in der gelockerten Krume (11-22 cm Bodentiefe), im Bereich der Pflugsohle (22-32 cm Bodentiefe) und unterhalb der Pflugsohle (33$43 \mathrm{~cm}$ Bodentiefe) möglich waren. Bei FMW wurden identische Beprobungstiefen gewählt. Die Untersuchungen sollten zeigen, ob die mechanische Bodenbelastung zu einer Verdickung der Wurzeln führte.

Auf gepflügtem Boden nahm bei 6x5t in 11-22 cm Bodentiefe der Anteil der Wurzeln mit höherem Durchmesser (200- >500 $\mu \mathrm{m})$ ab (Abb. 20 links unten). In 22-32 cm Bodentiefe entsprachen sich die Häufigkeitsverteilungen der Wurzeldurchmesser beider Belastungsstufen. In der Bodentiefe $33-43 \mathrm{~cm}$ wurde eine deutliche Zunahme der Wurzeln mit einem Durchmesser von 200-300 $\mu \mathrm{m}$ festgestellt. Die Ergebnisse deuten an, daß bei hoher Bodenbelastung der Wurzeldurchmesser in der Krume abgenommen hat. Unterhalb des Bearbeitungshorizontes in der Bodentiefe 33-43 cm führte der Lasteintrag dagegen zu einer Verdickung der Wurzeln.

Auf der Kontrollparzelle des Bodens mit FMW (Abb. 20 rechts oben) verlief die Häufigkeitsverteilung der Wurzeldurchmesser in den Bodentiefen 22-32 cm und 33-43 cm annähernd identisch. In der Bodenschicht 11-21 cm wurde ein höherer Anteil an Wurzeln mit einem Durchmesser von 100-300 $\mu \mathrm{m}$ und ein geringerer mit einem Durchmesser von 300-500 $\mu \mathrm{m}$ festgestellt. Durch den Lasteitrag von 6x5t wurde in der Bodenschicht 11-21 cm der Anteil der Wurzeln mit 100$200 \mu \mathrm{m}$ Durchmesser erheblich erhöht. Der Anteil der Wurzeln mit 200-300 $\mu \mathrm{m}$ 
Durchmesser nahm deutlich ab. Auch in der Bodenschicht 33-43 cm nahm die Anzahl der Wurzeln mit einem Durchmesser von 100-200 $\mu \mathrm{m}$ zu.

LBW
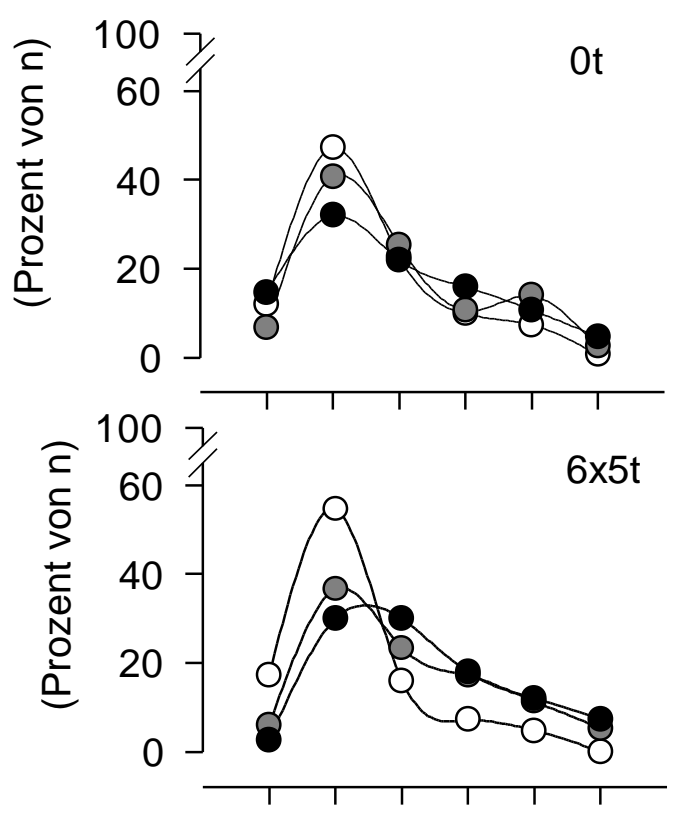

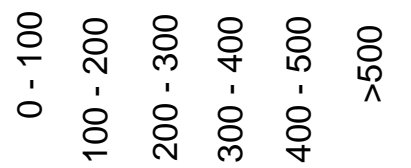

Wurzeldurchmesser $[\mu \mathrm{m}]$
FMW

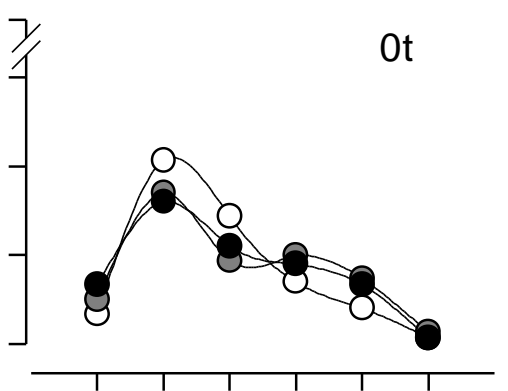

$6 \times 5 t$

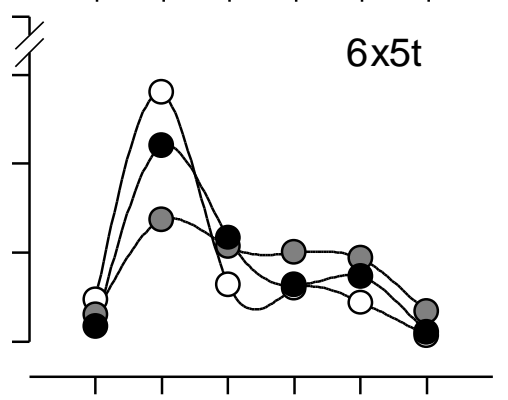

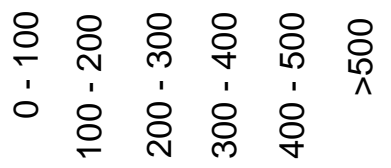

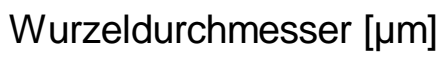

-O- Bodentiefe 11-22 cm

-O- Bodentiefe 22-32 cm

$\longrightarrow$ - Bodentiefe $33-43 \mathrm{~cm}$

Abb. 20: Wirkung der mechanischen Bodenbelastung und des Bearbeitungssystems auf den Wurzeldurchmesser von Winterweizen auf dem Standort Reinshof 1996. Die gemessenen Wurzeldurchmesser ( $n=150$ pro Bodentiefe) wurden in sechs Klassen eingeteilt. Die Beprobung wurde zum EC Stadium 45 in drei Bodentiefen durchgeführt.

\subsubsection{Standort Relliehausen}

Auf dem gepflügten Boden hatte die mechanische Bodenbelastung eine deutlich einschränkende Wirkung auf die WLD und die Durchwurzelungstiefe der Sommergerste (Abb. 21). Auf dem gepflügten Boden ohne Belastung betrug die GWL 5,05 km/m². Bei geringer und mittlerer Belastung erreichte die GWL ca. $60 \%$ der Kontrolle. Auf dem hoch belasteten Boden erreichte die GWL nur 44\% 
der Kontrolle. Die GWL im Unterboden wurde - im Vergleich zur Kontrolle - bei geringer bis hoher Bodenbelastung um ca. 60 \% verringert. Mit steigender Bodenbelastung nahm die maximale Durchwurzelungstiefe von $78 \mathrm{~cm}$ auf dem unbelasteten Boden auf $41 \mathrm{~cm}$ bei hoher Belastung ab. Das entspricht einem drastischen Verlust an durchwurzeltem Boden von annähernd $50 \%$.

LBW

1995

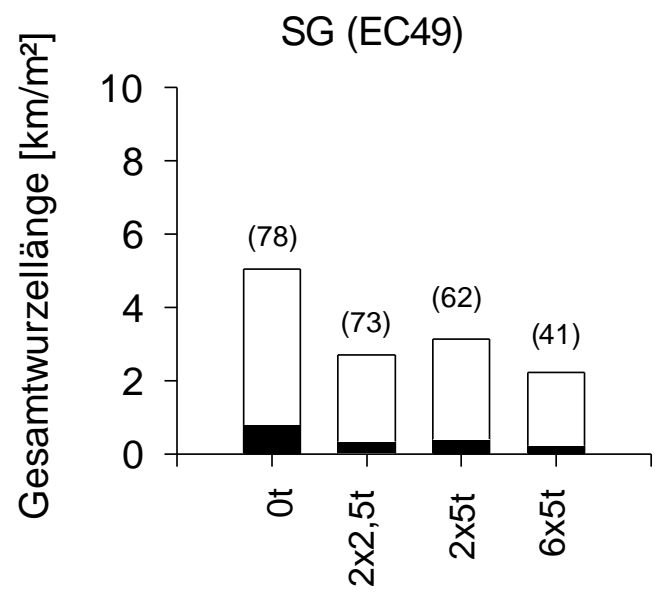

FMW

1995

SG (EC49)

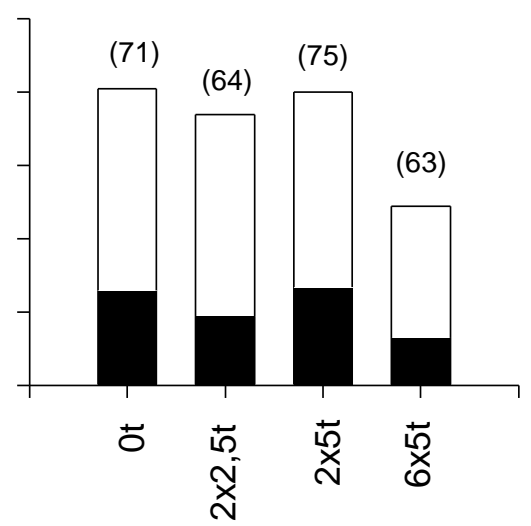

Abb. 21: Gesamtwurzellänge (GWL) und GWL in Oberboden (0-30 cm Bodentiefe, weiße Säulen) und Unterboden (von $30 \mathrm{~cm}$ Bodentiefe - maximale Durchwurzelungstiefe, schwarze Säulen) auf dem Standort Relliehausen. Dargestellt ist der Beprobungstermin 06.07.1995. Die angebauten Feldfrucht ist Sommergerste (SG). Die Zahlen in Klammern zeigen die maximale Durchwurzelungstiefe [cm].

Auf dem flach bearbeiteten Boden lag die GWL sowohl im Ober- als auch im Unterboden bei keiner bis mittlerer Belastung auf gleicher Höhe. Bei hoher Belastung wurde das Wurzelwachstum begrenzt: Die GWL des Oberbodens lag um $33 \%$, die GWL des Unterbodens um $46 \%$ unter den Werten der Kontrolle (FMW 0t). Die Durchwurzelungstiefe wurde weniger stark eingeschränkt.

Ein Vergleich der Bearbeitungssysteme - gemittelt über die Belastungsstufen zeigt beträchtliche Unterschiede: Die GWL bei FMW lag um den Faktor 2,3 höher als bei LBW. Bei Betrachtung der Unterbodendurchwurzelung wird der Unterschied noch deutlicher: Die Werte bei FMW lagen um den Faktor 5,2 höher als bei LBW. 


\subsubsection{Standort Großobringen}

Abb. 22 zeigt die GWL der Kulturpflanzen in den drei Versuchsjahren auf dem Standort Großobringen. Im ersten Versuchsjahr wurden die Wurzeln zu einem wesentlich späteren Termin in der Vegetation untersucht als an den anderen Standorten.

\section{LBW tief}

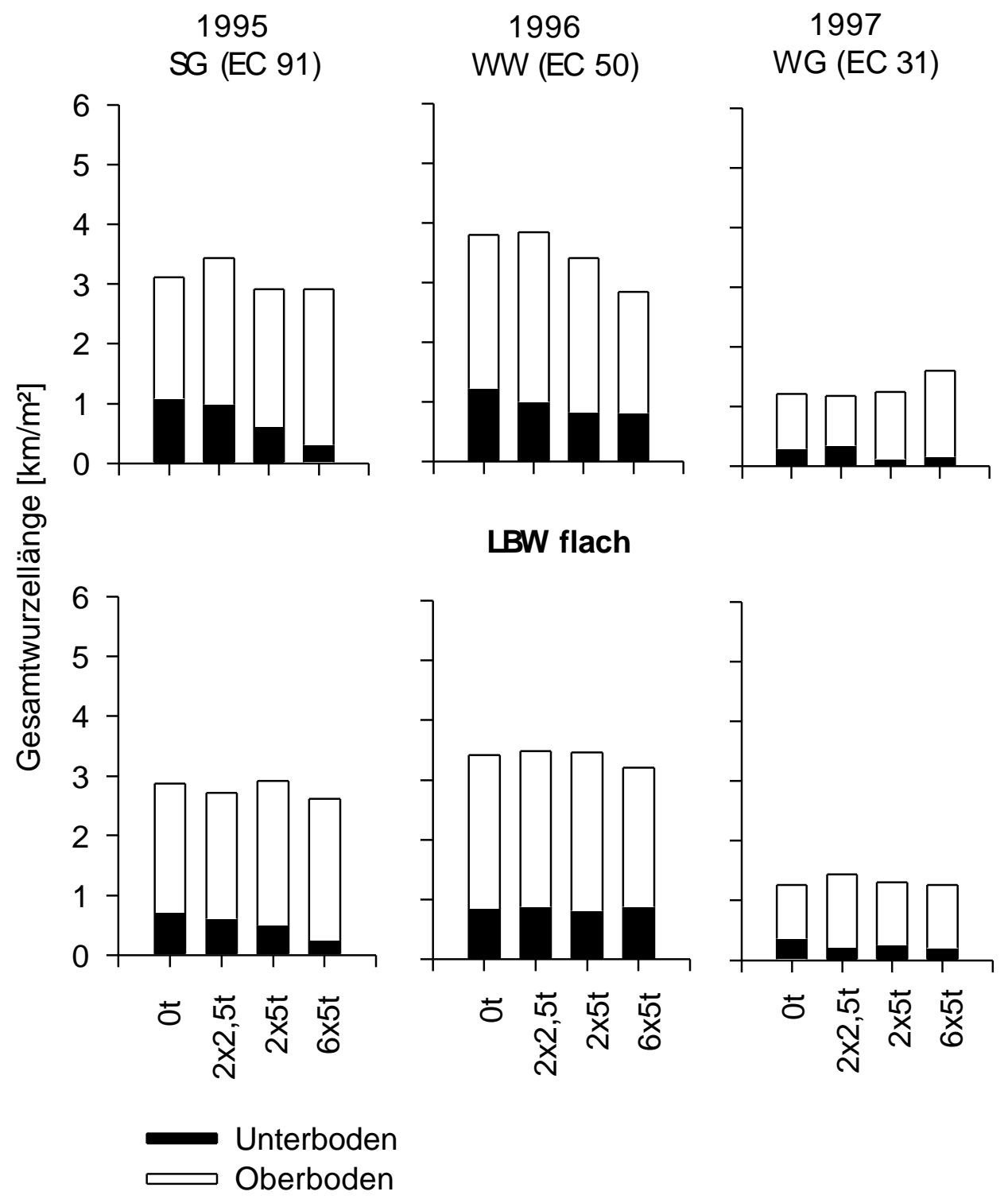

Abb. 22: Gesamtwurzellänge (GWL) und GWL in Oberboden (0-30 cm Bodentiefe, weiße Säulen) und Unterboden (von $30 \mathrm{~cm}$ Bodentiefe $-50 \mathrm{~cm}$ Durchwurzelungstiefe, schwarze Säulen) auf dem Standort Großobringen. Dargestellt sind die Beprobungstemine und die angebauten Feldfüchte: 08.08.1995, Sommergerste (SG); 17.06.1996 Winterweizen, (WW); 07.04.1997 Wintergerste, (WG). 
Unabhängig vom Bearbeitungsystem lag die GWL der Sommergerste 1995 bei allen Belastungsstufen auf etwa gleicher Höhe (Abb. 22 links). Sowohl die unterschiedliche Pflugtiefe bei LBW tief und LBW flach als auch die Höhe der Bodenbelastung hatten keine kennzeichnenden Auswirkungen auf die Gesamtlänge der Wurzelsysteme. Bei der GWL des Unterbodens zeigten sich deutliche Unterschiede: Bei beiden Bearbeitungssystemen nahm sie mit steigender Bodenbelastung ab. Wie Abb. 22 (links) zeigt, lagen die Werte der GWL des Unterbodens bei LBW flach um ca. $20 \%$ niedriger als bei LBW tief. Im zweiten Versuchsjahr lag die GWL des Winterweizens in den ersten beiden Belastungsstufen von LBW tief auf gleicher Höhe. Die GWL bei $2 \times 5 t$ lag um 10 $\%$ tiefer als bei der Kontrolle. Bei 6x5t lag die GWL um $25 \%$ unter der Kontrolle. Wie auch im ersten Versuchsjahr nahm die GWL des Unterbodens mit steigender Belastung ab: Bei der Kontrolle wurden im Unterboden 1,22 km Wurzellänge pro $\mathrm{m}^{2}$ gemessen.Bei hoher Belastung waren es nur noch 0,8 km pro

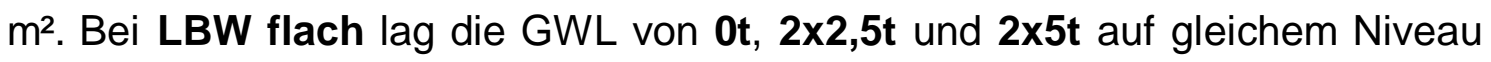
(Abb. 22 mitte). Die GWL bei 6x5t war um $12 \%$ geringer als bei der Kontrolle. Innerhalb der Belastungsstufen unterschied sich die GWL des Unterbodens nicht. Im dritten Versuchsjahr (Abb. 22 rechts) nahm bei LBW tief mit mittlerer und hoher Bodenbelastung die GWL im Vergleich zur Kontrolle (LBW tief 0t) zu, bei LBW flach erreichte die GWL bei allen Belastungsstufen annähernd die gleichen Werte. Im Unterboden konnte bei LBW tief und LBW flach eine geringfügige Reduktion der GWL bei steigender Bodenbelastung beobachtet werden. Diese Tendenz stellte sich bei LBW tief stärker dar als bei LBW flach. 


\subsubsection{Oberirdische Trockenmasse und Blattfläche}

Die mechanische Belastung eines Bodens über seine Eigenstabilität hinaus führt zu Gefügedeformationen. Diese Deformationen können die Speicher- und Leitungsfunktionen des Bodens als poröser Körper für Wasser und Gas stark einschränken. Eine mögliche Folge ist die Beeinträchtigung der physiologischen Wurzelfunktionen und des Wurzelwachstums. Geht man von einer belastungsabhängigen Reduktion des Wurzelsystems aus, so ist auch eine belastungsabhängige Beeinträchtigung des oberirdischen Pflanzenwachstums denkbar. Eine mögliche Ursache wäre eine verminderte Nährstoff- und Wasseraufnahme, oder eine Wachstumsdepression, die nicht direkt auf eine Mangelsituation in der Rhizosphäre zurückzuführen ist, vielleicht aber mit einer Reaktion des Hormonhaushaltes zu erklären wäre. Um eine auflastabhängige Beeinträchtigung des oberirdischen Pflanzenwachstums nachzuweisen, wurden Messungen der Trockensubstanz und der Blattfläche vorgenommen. Grundlage einer solchen Untersuchung ist die Erfassung der Produktion (oder Reduktion) der oberirdischen Trockenmasse und der Blattfläche eines Pflanzenbestandes über ein Zeitintervall (BEADLE 1993). Die Entwicklung der oberirdischen Trockenmasse spiegelt das Pflanzenwachstum unter den aktuellen Wachstumsbedingungen wider. Die Größe der Blattfläche bestimmt neben der Pigmentierung die Kapazität zur Licht- und $\mathrm{CO}_{2}$ - Aufnahme und damit zur Stoffproduktion. Die Blattfläche $\left[\mathrm{m}^{2}\right]$ bezogen auf die Bodenoberfläche $\left[\mathrm{m}^{2}\right]$ ergibt den Blattflächenindex (BFI). Der BFI nimmt bei den Getreidearten während des Schossens (EC 31-39) stark zu (zu den EC-Standien vgl. Tab 40, Anhang). Maximale Werte werden mit dem Ährenschieben, beziehungsweise kurz davor erreicht (EC 49/55). Danach sinkt der BFI kontinuierlich ab, was auf das Absterben älterer Blätter, am Halm von unten nach oben, zurückzuführen ist. Dieser Prozess wird dadurch gefördert, daß Blätter in den unteren „Blattetagen“ zunehmend beschattet werden und bald mehr Substanz veratmen als sie durch ihre Photosynthese gewinnen können.

Mit steigendem BFI in der Jugendentwicklung nimmt die aufgefangene Lichtmenge und damit die Substanzproduktion pro Zeiteinheit zu. Der maximale potentielle Ertragszuwachs, der bei den Getreidearten der gemäßigten Breiten 
ca. $25 \mathrm{~g}$ Trockenmasse pro $\mathrm{m}^{2}$ Bodenoberfläche und Tag betragen kann, wird im allgemeinen unter den Strahlungsbedingungen des (Früh)- Sommers bei einem BFI von 3-5 erreicht. Wird dieser „Schwellenwert des BFI“ überschritten, führt die Steigerung der Lichtinterzeption nur noch zu einer sehr geringen Zunahme der Substanzproduktion pro Zeiteinheit. Da eine maximale Substanzproduktion im Sinne des potentiellen Ertragszuwachses eine maximale Strahlungsinterzeption voraussetzt, sind Pflanzenbestände mit einem BFI weit unterhalb des "Schwellenwertes" in ihrer Substanzproduktion eingeschränkt. Die Größe der assimilierenden Blattfläche und deren Beständigkeit über die Zeit wird mit der Blattflächendauer (BFD) beschrieben. Die BFD ist das Integral des BFI über die Zeit (EHLERS 1996). In der vorliegenden Arbeit wird die BFD nach der Blüte bis zur mittleren Milchreife betrachtet. In diesem Entwicklungsabschnitt der Kornfüllung kann eine hohe BFD ein hohes Maß an eingelagerter Assimilate bedeuten.

\subsubsection{Standort Reinshof}

\subsection{Versuchsjahr 1995}

Im Versuchsjahr 1995 zeigte sich auf dem Standort Reinshof bei beiden Bearbeitungssystemen ein deutlicher Einfluß der Belastungsstufen auf die oberirdische Trockenmasse und die Blattfläche der Sommergerste (Abb. 23). Auf dem gepflügten Boden konnten die Pflanzen bis gegen Ende des Schossens auf dem Boden mit geringer und mittlerer Belastung nur ca. 50\% der oberirdischen Trockenmasse und der Blattfläche der Pflanzen auf der Kontrollparzelle (LBW 0t) bilden (Abb. 23 links). Die Wachstumsbedingungen bei hoher Bodenbelastung waren so ungünstig, daß die Sommergerste im Vergleich zur Kontrolle $70 \%$ weniger oberirdische Trockenmasse und Blattfläche ausbildete. Auf dem gepflügten Boden war mit steigender Bodenbelastung die Produktion von Trockenmasse und Blattfläche zunehmend eingeschränkt.

Bei FMW (Abb. 23 rechts) bildeten die Pflanzen mit keiner, geringer und mittlerer Bodenbelastung auf gleichem Niveau mehr oberirdische Trockenmasse als die Pflanzen auf dem hoch belasteten Boden. Die Entwickung der Blattfläche zeigte die gleiche Tendenz: Zu den Entwicklungsstadien EC 25 und EC 30 lag 
der BFI bei keiner, geringer und mittlerer Bodenbelastung um ca. 0,6 m²/ $\mathrm{m}^{2}$ höher als hoher Belastung. Zum Ende des Schossens betrug die Differenz zwischen den ersten drei Belastungsstufen und der hoch belasteten Variante ca. $1 \mathrm{~m}^{2} / \mathrm{m}^{2}$. Sowohl bei der Trockenmasse als auch bei der Blattfläche war das $\mathrm{Ni}$ veau der Belastungsstufen 2x2,5t und 2x5t bei FMW gleich dem der Kontrollvariante (FMW 0t). Auf dem flach bearbeiteten und gemulchten Boden wurde die oberirdische Trockenmasse und die Blattfläche nur in der Variante mit hoher Bodenbelastung deutlich eingeschränkt. Ausgesät wurde die Sommergerste direkt nach Versuchsbeginn am 13. April 1995. Der späte Aussaattermin verkürzte die Vegetationsperiode, so daß die einzelnen Wachstumsabschnitte schnell durchlaufen wurden. Möglicherweise ist das der Grund für die insgesamt geringe Trockenmasse- und Blattflächenbildung der Sommergerste auch auf den unbelasteten Parzellen.
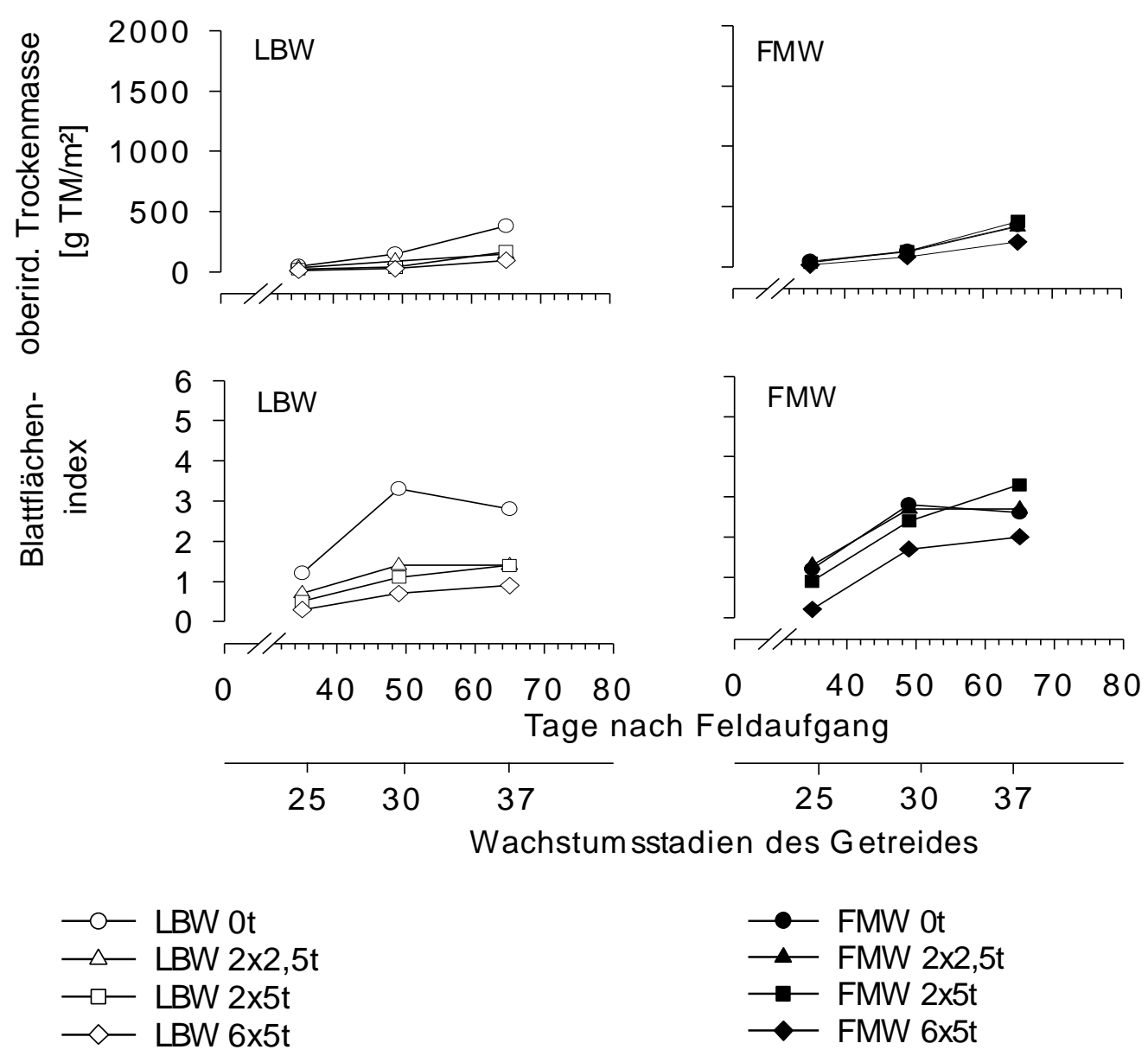

Abb. 23: Entwicklung der oberirdischen Trockenmasse (oben) und des Blattflächenindexes (unten) von Sommergerste über die Zeit auf dem Standort Reinshof im Versuchsjahr 1995. Der Termin des Feldaufgangs geht aus Tab. 57 hervor. Dargestellt sind Ergebnisse von Block 2. 
Tendenziell erreichte der Bestand auf dem gepflügten Boden bei mittlerer und hoher Bodenbelastung zu EC 61 nicht die Höhe des Bestandes bei fehlender und geringer Belastung: Die Bestandeshöhe bei 2x5t war um 20\% niedriger als bei 0t, bei 6x5t betrug die Differenz zur Kontrolle (LBW 0t) 30\% (Abb. 24).

Auf dem flach bearbeiteten Boden war zu EC 31 - wie auf dem gepflügten Boden - die Bestandeshöhe bei allen Belastungsstufen in etwa gleich. Zu EC 61 erreichte der Bestand mit hoher Bodenbelastung 80\% der Bestandeshöhe der Kontrolle (FMW 0t).

Beeinträchtigungen des Längenwachstums durch die mechanische Bodenbelastung traten bei LBW 2x5t und 6x5t auf (Abb. 24). Bei FMW war das Längenwachstum nur bei $6 \times 5 t$ eingeschränkt. Die Bestandeshöhe auf den Bearbeitungssystemen zeigte eine ähnliche Reaktion auf die Belastungsstufen wie die Trockenmasse und die Blattfläche (Abb. 23).

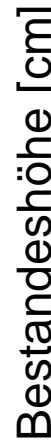

$\frac{E}{0}$
$\frac{0}{0}$
$\frac{0}{0}$
$\frac{0}{0}$
$\frac{0}{0}$
$\frac{0}{0}$
0
0
0

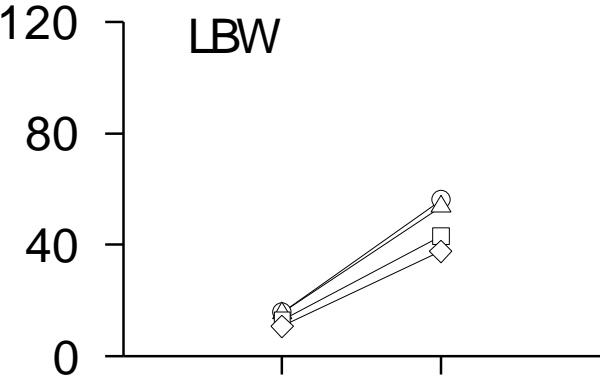

31

61

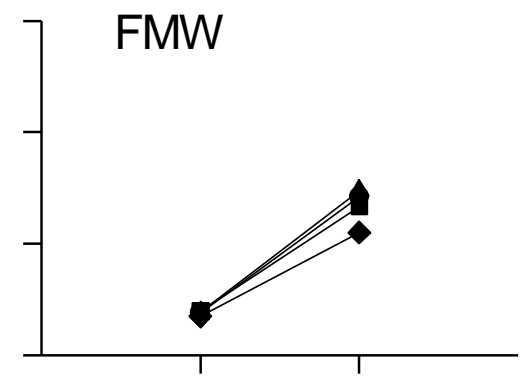

31

61

Entwicklungsstadien des Getreides

$$
\begin{aligned}
& \multimap-\text { LBW 0t } \\
& \square-\text { LBW 2x2,5t } \\
& \square-\text { LBW 2x5t } \\
& \neg \text { LBW 6x5t }
\end{aligned}
$$

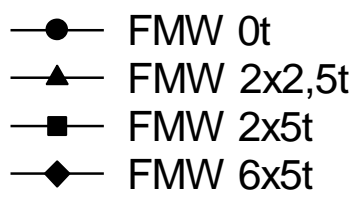

Abb. 24: Bestandeshöhe der Sommergerste auf dem Standort Reinshof 1995 zu den Entwicklungsstadien EC 31 und EC 61. Dargestellt sind arithmetische Mittelwerte aus Block 1-4. 


\subsection{Versuchsjahr 1996}

Die oberirdische Trockenmasse des Winterweizens zeigte bei gepflügtem Boden bis EC 75 keine belastungsabhängigen Unterschiede (Abb.25). Während der Reife lag die Trockenmasse der Kontrolle (LBW 0t) deutlich höher als in den belasteten Parzellen (Abb. 25 links).
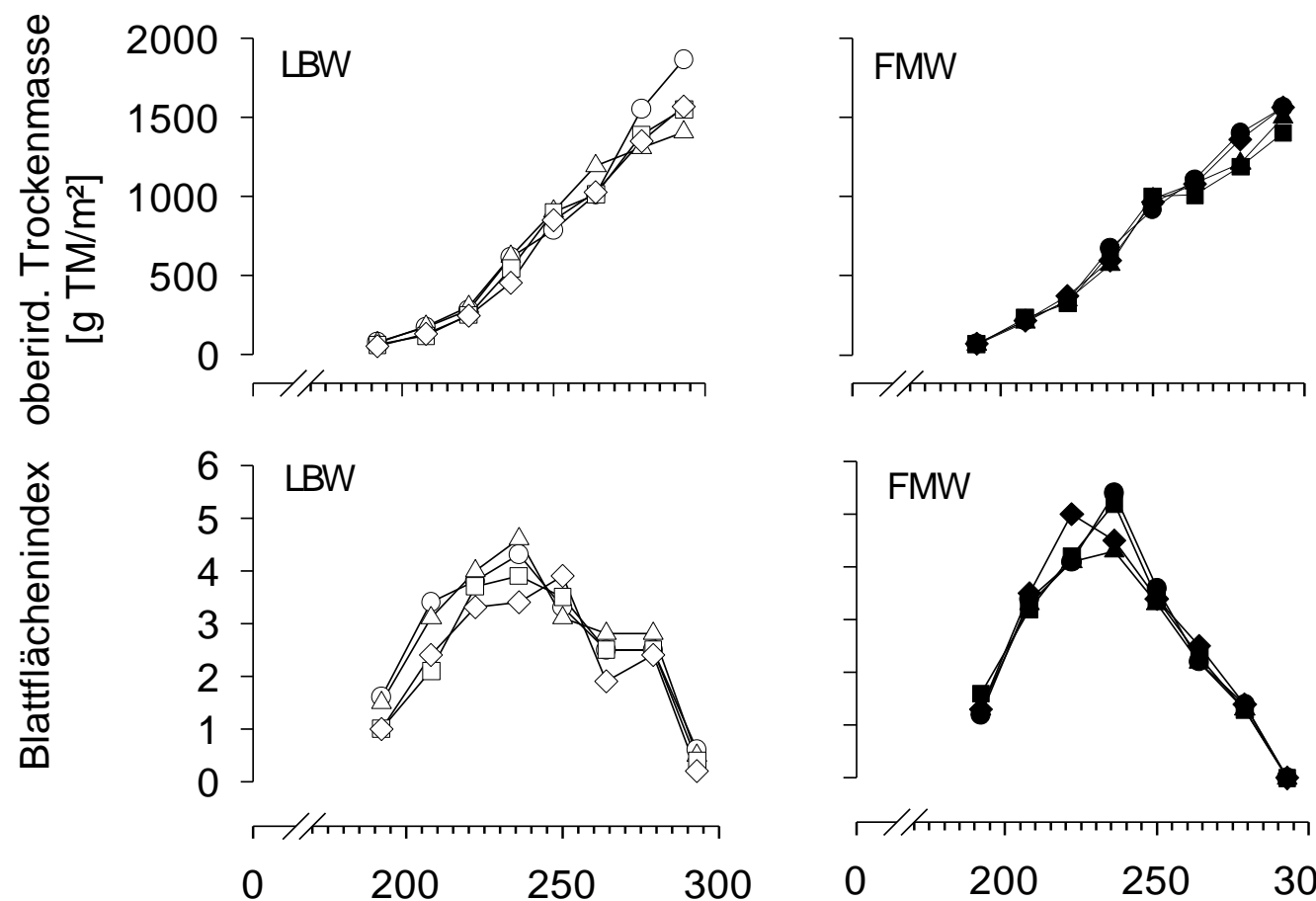

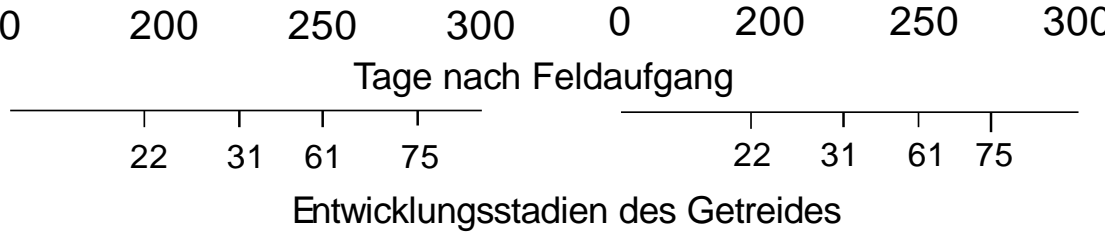

$$
\begin{aligned}
& \multimap \text { LBW 0t } \\
& \neg-\text { LBW } 2 \times 2,5 \mathrm{t} \\
& \multimap-\text { LBW } 2 \times 5 \mathrm{t} \\
& \neg-\text { LBW } 6 \times 5 \mathrm{t}
\end{aligned}
$$

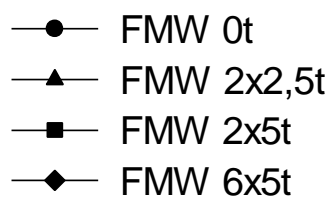

Abb. 25: Entwicklung der oberirdischen Trockenmasse (oben) und des Blattflächenindexes (unten) von Winterweizen über die Zeit auf dem Standort Reinshof im Versuchsjahr 1996. Dargestellt sind Ergebnisse aus Block 2.

Bei der Blattfläche zeigten sich Unterschiede von Anfang an: Auf der Kontrollparzelle (LBW 0t) und bei geringer Belastung bildete sich zu Vegetationsbe- 
ginn eine um ca. $0,8 \mathrm{~m}^{2} / \mathrm{m}^{2}$ größere Blattfläche als bei mittlerer und hoher Bodenbelastung. Auf dem gepflügten Boden setzte die alterungsbedingte Reduktion der Blattfläche bei $\mathbf{0 t}$, 2x2,5t und 2x5t vor EC 61 ein. Bei $6 \times 5 t$ nahm die Blattfläche bis zum Beginn der Blüte (EC 61) zu. Nach der Blüte nahm die Blattfläche bei hoher Belastung stärker und schneller ab als bei den anderen Belastungsstufen.

Bei FMW (Abb. 25 rechts) verlief sowohl die Trockenmassezunahme als auch die Entwicklung der Blattfläche über die gesamte Vegetation bei allen Belastungsstufen in etwa gleich. Abweichend davon setzte bei $6 \times 5 t$ die Reduktion der Blattfläche ca. 10 Tage früher ein als bei 0t, 2x2,5t und 2x5t.

Auf dem gepflügten Boden und bei hoher Belastung lag die BFD um 10\% niedriger als bei den Belastungsstufen 0t, 2x2,5t und 2x5t. Auf denm flach bearbeiteten Boden kehrte sich diese Tendenz um: Die BFD bei 6x5t lag um 7\% höher als bei den anderen Belastungsstufen (Abb. 26). Insgesamt lag die BFD bei FMW auffallend niedriger als bei LBW.

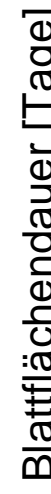

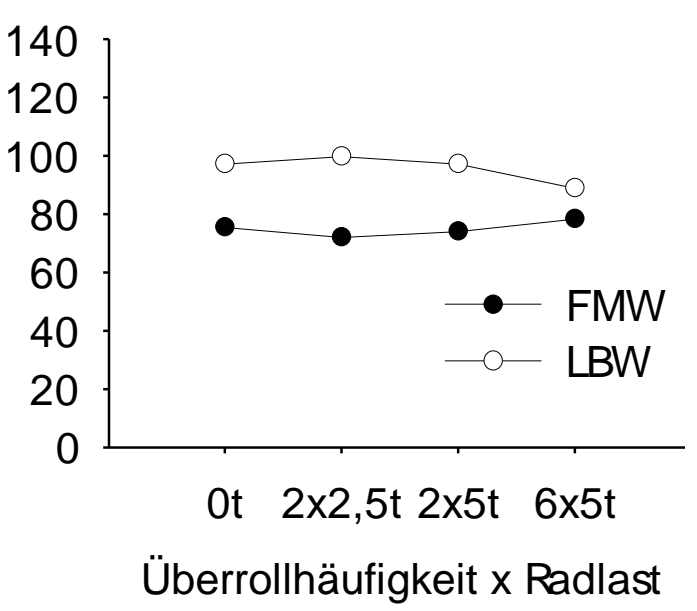

Abb. 26: Blattflächendauer (BFD) des Winterweizens vom Ende der Blüte (EC 69) bis zur mittleren Milchreife (EC 75) auf dem Standort Reinshof 1996 in Abhängigkeit von der Belastungsstufe. Dargestellt sind Ergebnisse aus Block 2.

Abb. 27 zur Bestandeshöhe zeigt zum Schoß- und Blühbeginn annähernd identische Werte bei beiden Bearbeitungssystemen und allen Belastungsstufen. Die Bestandeshöhe bei LBW 6x5t lag zum Stadium EC 61 ca. 10\% unter der Höhe bei LBW 0t, 2x2,5t und 2x5t. 

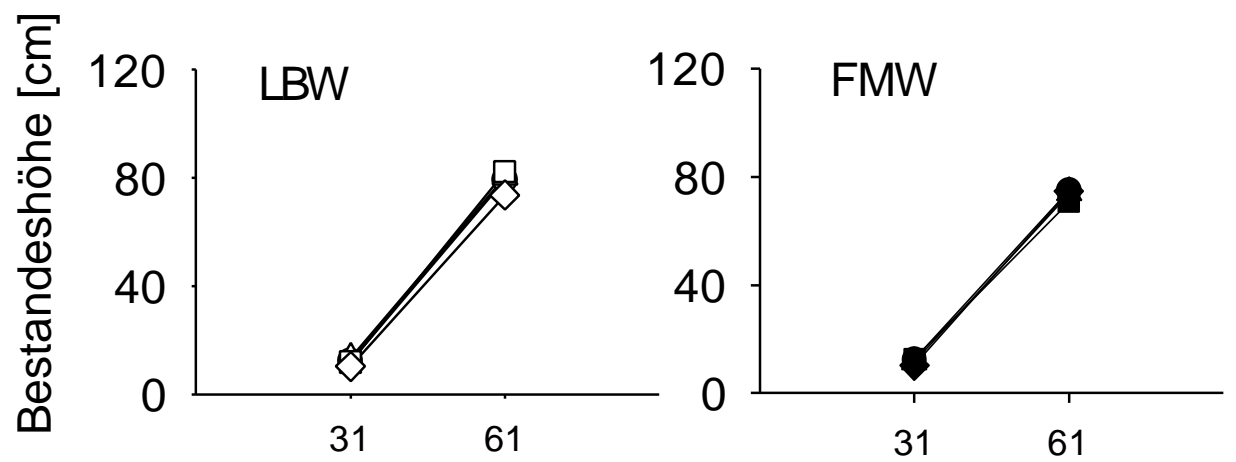

Entwicklungsstadien des Getreides
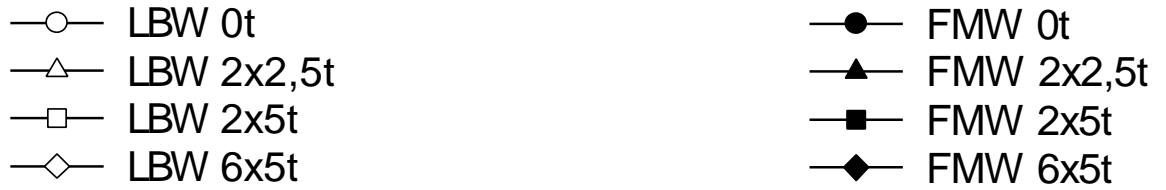

Abb. 27: Bestandeshöhe des Winterweizens auf dem Standort Reinshof 1996 zu den Entwicklungsstadien EC 31 und EC 61. Dargestellt sind arithmetische Mittelwerte aus Block 1-4.

Die mechanische Bodenbelastung zu Versuchsbeginn 1995 beeinträchtigte das Längenwachstum des Winterweizens 1996 nur bei LBW 6x5t. Bei FMW konnte keine Einschränkung nachgewiesen werden.

\subsection{Versuchsjahr 1997}

Auf dem Standort Reinshof trat im Frühjahr 1997 bei FMW ein hoher Konkurrenzdruck durch Windhalm (Apera spica-venti (L.)) auf. 192 Tage nach Feldaufgang der Wintergerste wurde auf beiden Bearbeitungssystemen eine Windhalmbekämpfung mit Ralon Super (36 g/ha Fenoxaprop-ethyl, s. Tab. 57) durchgeführt. Diese Maßnahme führte nicht zu einer ausreichenden Reduktion der Windhalmpopulation. 202 Tage nach Feldaufgang wurde als zweite Maßnahme, wiederum bei beiden Bearbeitungssystemen, Isoproturon (1000 g/ha) eingesetzt. Der wiederholte Herbizideinsatz unterdrückte das Windhalmwachstum bei FMW zufriedenstellend, beeinträchtigte aber bei beiden Bearbeitungssyetmen die Entwicklung der Wintergerste (Abb. 28). 

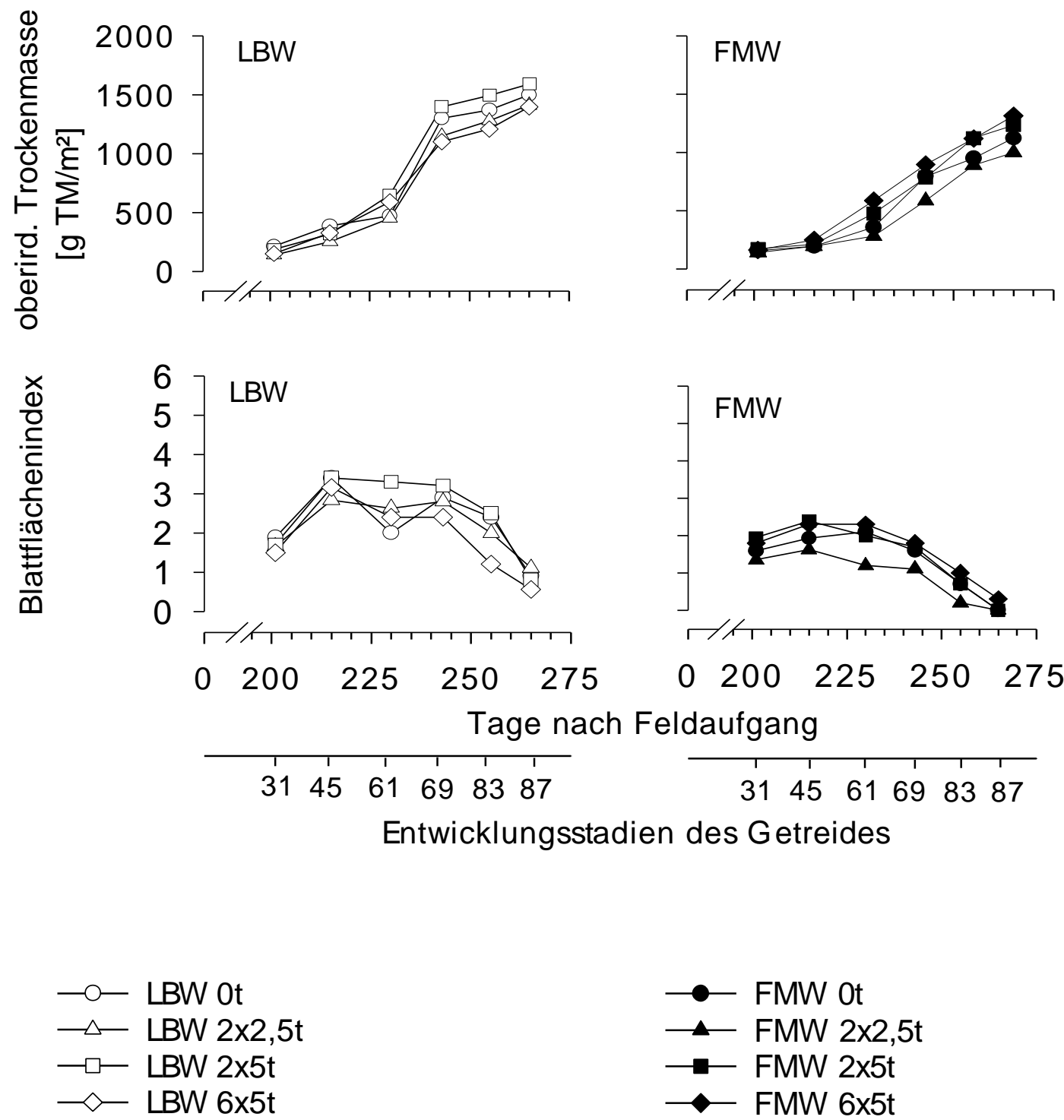

Abb. 28: Entwicklung der oberirdischen Trockenmasse (oben) und des Blattflächenindexes (unten) von Wintergerste auf dem Standort Reinshof über die Zeit im Versuchsjahr 1997. Dargestellt sind Ergebnisse aus Block 3.

Der maximale Blattflächenindex von Getreide wird in der Literatur im Mittel mit ca. 6 angegeben (GeISLER 1983; STÜLPNAGEL 1979). Bei LBW (Abb. 28 links) wurde ein maximaler Blattflächenindex um 3,5 erreicht. Da auf dem gepflügten Boden keine Windhalmkonkurrenz auftrat, wird die belastende Wirkung des intensiven Herbizideinsatzes als Ursache der schwachen Blattflächenentwicklung angenommen.

Nicht nur die Größe der Blattfläche, sondern auch die Blattflächenentwicklung war untypisch: Bei Wintergetreide erreicht die Blattfläche zu EC 45-55 ihr Ma- 
ximum und nimmt dann stetig ab (GEISLER 1983). Auf dem gepflügten Boden war die maximale Blattfläche zu EC 45 erreicht und nahm dann bis EC 61 ab. In der Blüte (EC 61-69) kam es zu einem Wachstumsschub - die Blattfläche nahm noch einmal zu. Nach diesem zweiten Anstieg setzte die Phase der alterungsbedingten Blattflächenreduktion ein. Möglicherweise ist die Wachstumsbeeinträchtigung vor der Blüte durch den zweimaligen, späten Herbizideinsatz der Grund für den untypischen Wachstumsschub in der Blüte. Dies ist besonders deutlich in Abb.28 (links) bei LBW 0t zu beobachten. Ab EC 45 zeigte sich bei den vier Belastungsstufen ein unterschiedlicher Verlauf der Trockenmasse und der Blattfläche. Bei 0t war die Beeinträchtigung der Massezunahme stärker als bei den anderen Belastungsstufen (EC 45-61). Nach der Blüte (EC 69) konnte bei 2x5t die höchste Trockenmasse pro Fläche festgestellt werden. Auf der Kontrolle hatten die Pflanzen eine etwas höhere Masse pro Fläche als bei 2x2,5t und 6x5t. Bei der Blattfläche zeigte sich ein ähnlicher Verlauf. Bis EC 45 lagen alle Belastungsstufen auf gleicher Höhe. Die Wachstumsbeeinträchtigung vor der Blüte (bis EC 61) war bei $0 t$ am stärksten und bei $2 \times 5 t$ am schwächsten. Im zweiten Wachstumsschub (EC 61-69) stieg die Blattfläche bei ot stärker an als bei den anderen Belastungsstufen, erreichte aber nicht die Fläche von 2x5t. Die Blattfläche bei 6x5t stieg nach der Wachstumsdepression nicht wieder an und blieb bis zum Ende der Messungen unter der Blattfläche der anderen Belastungsstufen.

Bei FMW (Abb. 28 rechts) war der Ungrasdruck selbst nach zweimaligem Herbizideinsatz bei 0t gering (50-100 Rispen/m²), bei $2 \times 2,5 t$ sehr hoch (>300 Rispen $/ \mathrm{m}^{2}$ ) und bei 2x5t und 6x5t mäßig (150-200 Rispen $/ \mathrm{m}^{2}$ ). Der für die einzelnen Belastungsstufen angegebene Ungrasdruck wiederholte sich auf den Blökken 1-4. Es kam zu einer erheblichen Wachstumsdepression als Folge einer kombinierten Wirkung des Konkurrenzdruckes durch den Windhalm und der Belastung durch den Herbizideinsatz: Die maximal erreichten Blattflächenindizes lagen unter 2,5.

Bis EC 45 war das Pflanzengewicht und die Blattfläche auf allen Belastungsstufen von FMW in etwa gleich. Ab EC 61 lag das Gewicht und die Blattfläche bei 6x5t höher als auf der Kontrolle (FMW 0t). Bei 2x2,5t - der Belastungs- 
stufe mit dem stärksten Windhalmdruck - wurde die geringste Trockenmasse und die geringste Blattfläche gemessen.

Die Höhe der BFD zeigt erhebliche Unterschiede zwischen den Bearbeitungssystemen und den Belastungsstufen (Abb. 29)

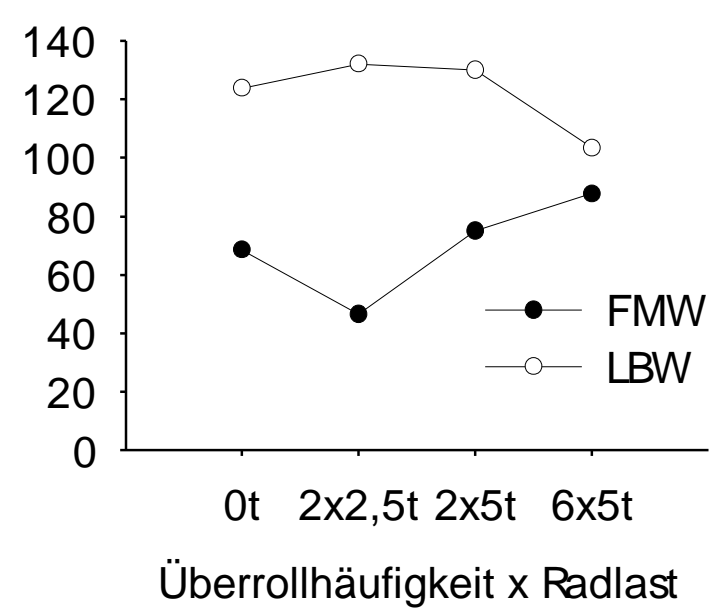

Abb. 29: Blattflächendauer (BFD) der Wintergerste vom Ende der Blüte (EC 69) bis zur Gelbreife (EC 87) auf dem Standort Reinshof 1997 in Abhängigkeit von der Belastungsstufe. Dargestellt sind Ergebnisse aus Block 3.

Bei LBW lag die BFD bei 6x5t um 20\% unter der mittleren BFD der anderen drei Belastungsstufen. Bei FMW 2x2,5t wurde die geringste BFD gemessen. Sie lag 32\% unter dem Wert der Kontrolle (FMW 0t). Die BFD auf der maximal belasteten Parzelle (6x5t) lag um 30\% über der BFD der Kontrolle (FMW 0t). Der hohe Konkurrenzdruck durch den Windhalm beeinträchtigte das Blattwachstum nur bei FMW. Hier lag die mittlere BFD um 44\% unter der von LBW. Die Entwicklung der Bestandeshöhe von EC 31 bis EC 61 zeigte einen identischen Verlauf bei LBW 0t, 2x2,5t, 2x5t einerseits und bei FMW über alle Belastungsstufen andererseits (Abb. 30). Der Bestand bei LBW 6x5t erreichte zu EC 61 82\% der Höhe des Bestandes auf der Kontrolle (LBW 0t). Die mittlere Bestandeshöhe bei FMW lag zu EC 61 ca. 14\% unter der mittleren Höhe bei LBW. 


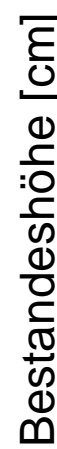

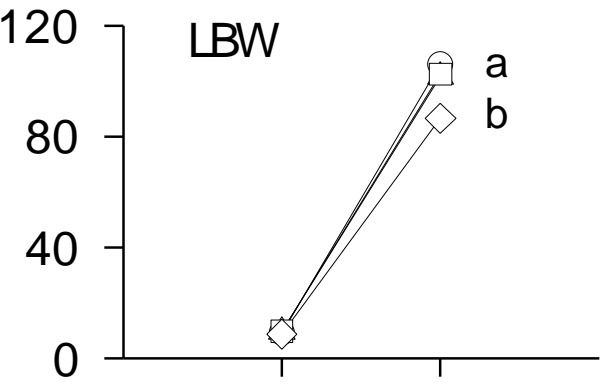

$31 \quad 61$

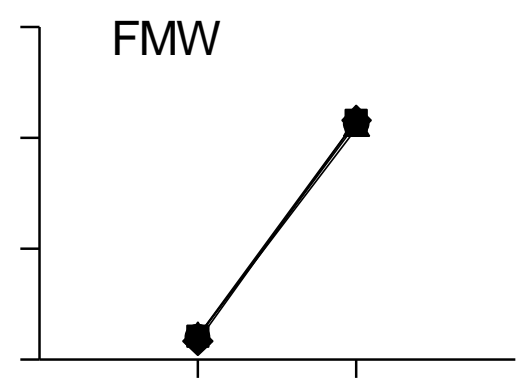

31

61

$$
\begin{aligned}
& \multimap-\text { LBW 0t } \\
& \neg-\text { LBW 2x2,5t } \\
& \square-\text { LBW 2x5t } \\
& \neg \text { LBW 6x5t }
\end{aligned}
$$

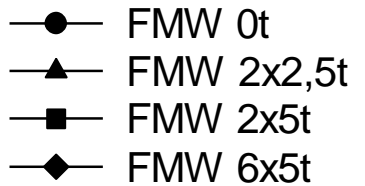

Abb. 30: Bestandeshöhe der Wintergerste auf dem Standort Reinshof $1997 \mathrm{zu}$ den Entwicklungsstadien EC 31 und EC 61. Dargestellt sind arithmetische Mittelwerte aus Block 1-4. Ungleiche Buchstaben kennzeichnen signifikante Unterschiede innerhalb eines Bearbeitungssystems. 


\subsubsection{Standort Relliehausen}

\subsection{Versuchsjahr 1995}

Bei beiden Bearbeitungssystemen zeigten Trockenmasse und Blattfläche einen unterschiedlichen Verlauf abhängig von der Belastungsstufe und dem Entwicklungsstadium der Sommergerste (Abb. 31). Ein Vergleich der Bearbeitungssysteme zeigte keine kennzeichnenden Unterschiede. Die Bestände auf beiden Systemen erreichten annähernd die gleichen Trockengewichte und ähnlich hohe BFI.
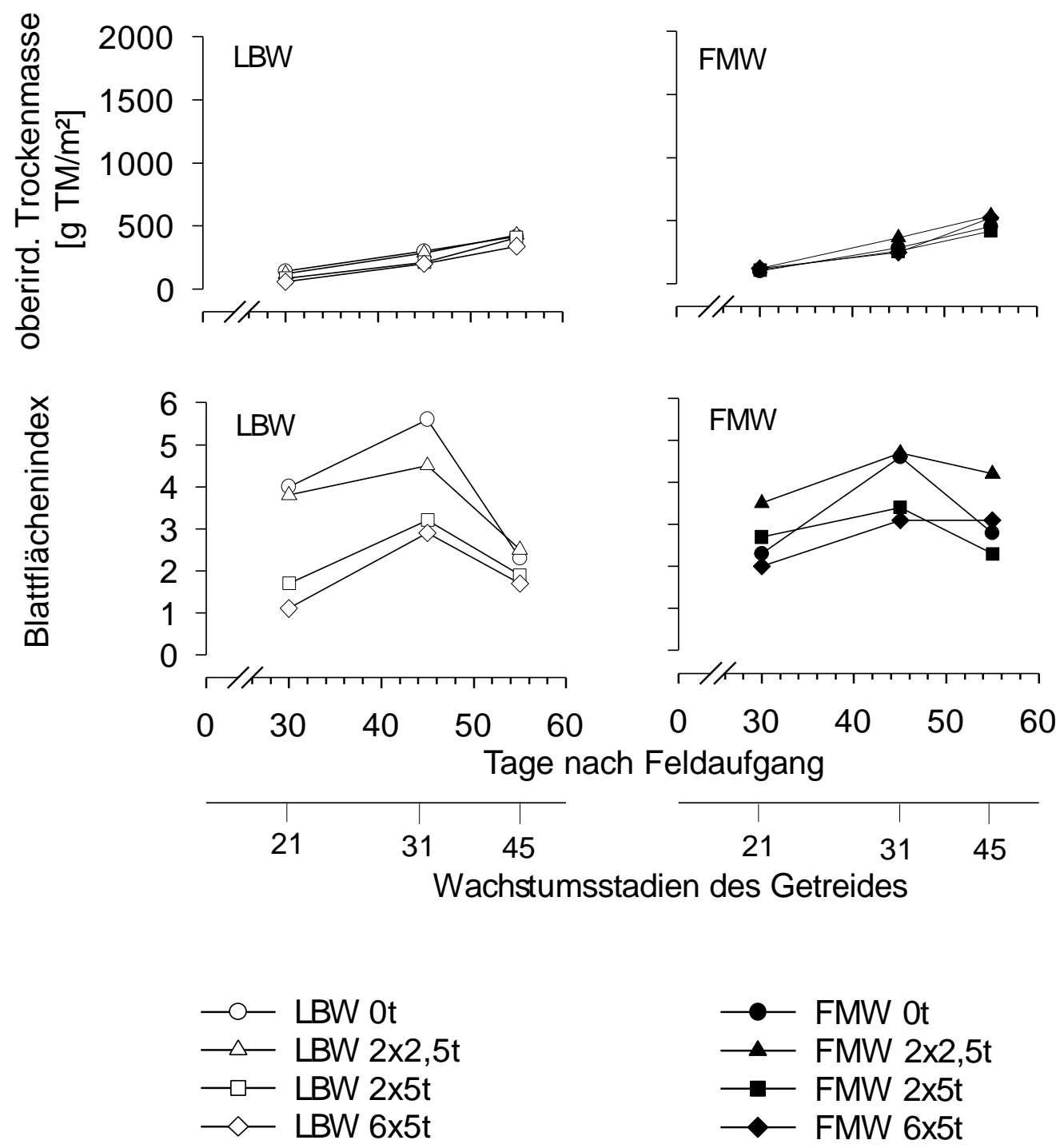

Tage nach Feldaufgang

3145

$21 \quad 31 \quad 45$

Wachstumsstadien des Getreides

Abb. 31: Entwicklung der oberirdischen Trockenmasse (oben) und des Blattflächenindexes (unten) von Sommergerste auf dem Standort Relliehausen über die Zeit im Versuchsjahr 1995. 
Auf dem gepflügten Boden (Abb. 31 links) stand zu Beginn der Bestockung bei Ot (BFI 4) und 2x2,5t (BFI 3,8) mit einem BFI über 3 mehr Blattfläche zu Verfügung, um eingestrahlte Energie aufzufangen und zur Substanzproduktion umzusetzen. Bei 2x5t und $6 \times 5 t$ lag der BFI zum selben Zeitpunkt unter 2,0. Wie der Verlauf der Trockenmassebildung zeigt, reichte bei $2 \times 5 t$ und $6 \times 5 t$ die Blattfläche nicht aus, um eine Stoffproduktion in der Höhe von 0t und 2x2,5t zu erreichen. Zu EC 31 (Abb. 31 links) lag die gemittelte Trockenmasse von $2 \times 5 \mathbf{t}$ und $6 \times 5 \mathrm{t}$ um $40 \%$ unter der gemittelten Trockenmasse von $0 t$ und $2 \times 2,5 \mathrm{t}$. $\mathrm{Zu}$ diesem Zeitpunkt zeigte auch die Blattfläche geringere Werte mit steigender Belastung: Auf dem Boden mit geringer Belastung lag die Blattfäche um 20\% unter der Kontrolle (LBW 0t), bei mittlerer und hoher Bodenbelastung um etwa $40 \%$ unter dem Wert der Kontrolle (LBW 0t). Zu EC 45 war der BFI bei allen Belastungsstufen bereits stark abgefallen. Zu diesem Stadium war der BFI bei 2x5t und $6 \times 5 \mathrm{t}$ um $25 \%$ kleiner als der BFI bei 0 t und $2 \times 2,5 t$.

Auf dem flach bearbeiteten und gemulchten Boden (Abb. 31 rechts) lag zu EC 21 nur der BFI von 2x2,5t über 3. Bei dieser Belastungsstufe lag die Höhe der produzierten Trockenmasse von EC 21 bis EC 45 über den Werten der anderen Belastungsstufen. Bis EC 31 stieg der BFI bei $2 \times 5 t$ und $6 \times 5 t$ auf knapp über 3 . Bei 0t und 2x2,5t erreichte der BFI 4,5. Auffällig ist der starke Anstieg des BFI bei $\mathbf{0 t}$ von EC 21 bis EC 31. Zu EC 45 hatte der BFI bei $\mathbf{0 t}, \mathbf{2 \times 2 , 5 t}$ und $\mathbf{2 \times 5 t}$ abgenommen. Bei 6x5t blieb der BFI von EC 31 bis EC 45 auf dem gleichen Niveau - die grüne Blattfläche wurde nicht reduziert. Möglicherweise wurde die hohe Dauerhaftigkeit der assimilierenden Blattfläche zu einer verstärkten Substanzproduktion genutzt: Die Zunahme an Trockensubstanz von EC 31 bis EC 45 betrug auf bei hoher Belastung $17 \mathrm{~g}$ Trockenmasse pro $\mathrm{m}^{2}$ Bodenoberfläche und Tag, bei den anderen Belastungsstufen betrug die Zunahme dagegen nur $11 \mathrm{~g}$ Trockenmasse pro $\mathrm{m}^{2}$ Bodenoberfläche und Tag.

Bei LBW 0t, 2x2,5t und FMW 2x2,5t zeigt Abb. 31 zu EC 21 und 31 sehr hohe Blattflächenindizes. Möglicherweise sind die hohen Werte auf Meßfehler zurückzuführen. 


\subsection{Versuchsjahr 1996}

Die Entwicklung der Trockenmasse und der Blattfläche des Winterweizens auf dem Standort Relliehausen im Versuchsjahr 1996 ist in Abb. 32 dargestellt.
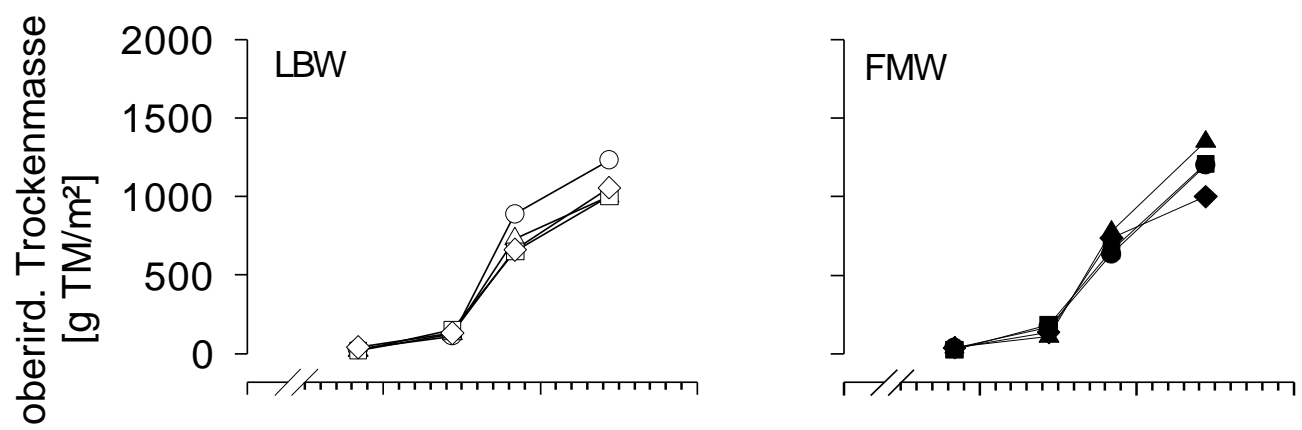

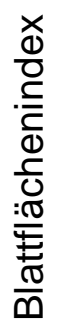
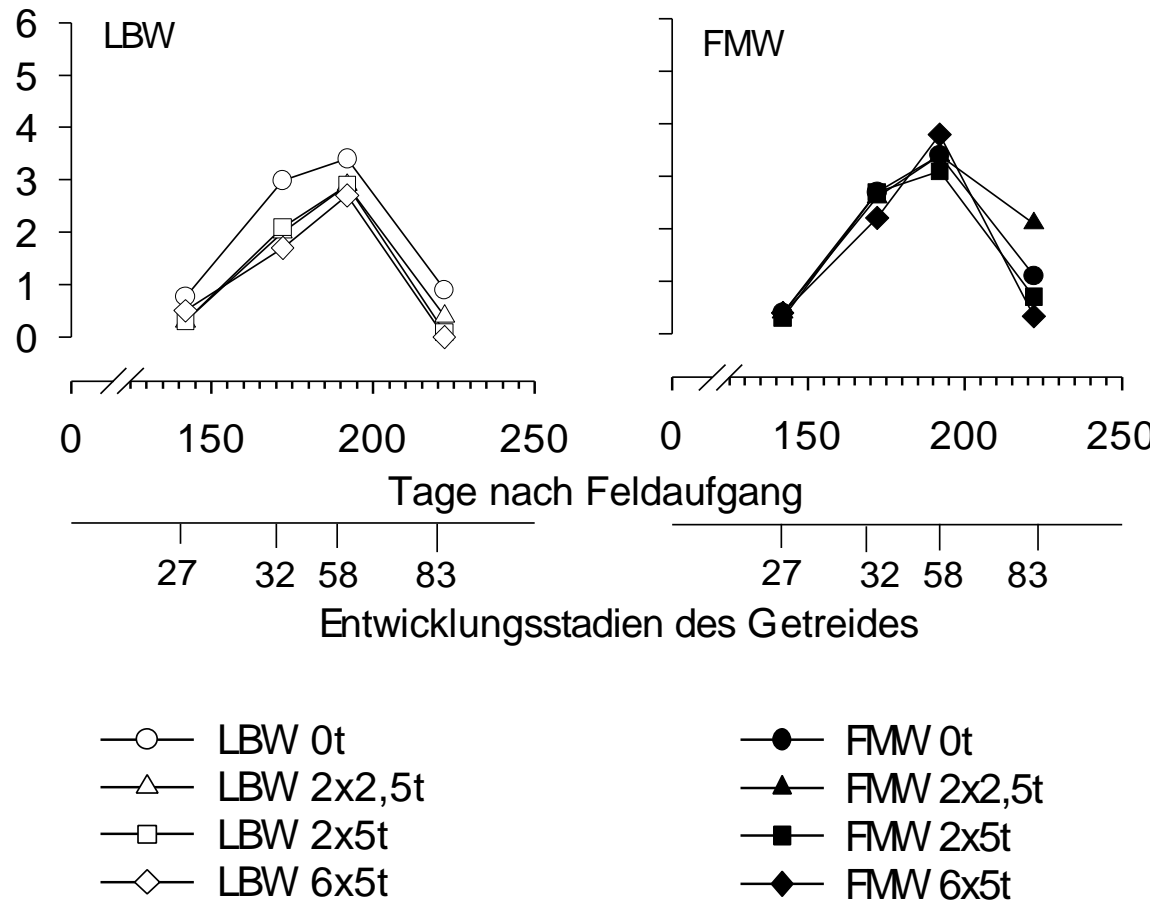

250

$150200 \quad 250$

Tage nach Feldaufgang
27
twicklungsstadien des Getreides

$3258 \quad 83$

Abb. 32: Entwicklung der oberirdischen Trockenmasse (oben) und des Blattflächenindexes (unten) von Winterweizen auf dem Standort Relliehausen über die Zeit im Versuchsjahr 1996.

Bei LBW (Abb. 32 links) erreichte der Bestand auf der unbelasteten Parzelle zu EC 32 einen BFI über 3. Bei den anderen Belastungsstufen lag der BFI zum selben Zeitpunkt bei 2. Die größere Blattfläche bei keiner Belastung führte zu einer geringfügig höheren Trockenmassebildung: Von EC 32 bis EC 58 betrug der Trockenmassezuwachs auf dem Boden ohne Belastung $25 \mathrm{~g}$ Trockenmasse pro $\mathrm{m}^{2}$ Bodenoberfläche und Tag. Der gemittelte Trockenmassezuwachs bei geringer, mittlerer und hoher Belastung lag bei $22 \mathrm{~g}$ Trockenmasse pro $\mathrm{m}^{2}$ Bodenoberfläche und Tag. Von EC 58 bis zum Ende der Messungen (EC 83) 
lag das Trockengewicht der Pflanzen auf der Kontrolle (LBW 0t) um ca. 15\% über dem gemittelten Trockengewicht der Parzellen mit Bodenbelastung.

Bei FMW (Abb. 32 rechts) wies die Entwicklung des Trockengewichtes und der Blattfläche bei allen Belastungsstufen bis zum Beginn der Blüte kaum Unterschiede auf. Ab EC 58 nahm bei allen Belastungsstufen der BFI alterungsbedingt ab. Diese Abnahme der Blattfläche fiel bei 2x2,5t am geringsten aus: Zu EC 83 war der BFI bei 2x2,5t um 195\% höher als der gemittelte BFI von 0t, 2x5t und 6x5t. Die größere Blattfläche führte zu einem höheren Trockengewicht der Pflanzen. Auffällig ist der Kurvenverlauf der Trockenmasse als Funktion der Zeit bei der hoch belasteten Variante (FMW): Hier war der tägliche Trockenmassezuwachs in der Zeit von EC 58 bis EC 83 um 54\% geringer als bei der Kontrolle (FMW 0t). Der BFI zeigte bei dieser Belastungsstufe den stärksten Rückgang der Blattfläche im Alterungsprozess. Die BFD des Winterweizens (Abb. 33) nahm auf dem gepflügten Boden im beobachteten Zeitabschnitt mit steigender Bodenbelastung ab. Bei FMW war eine solche Beziehung nicht festzustellen. Da sich bei FMW die alterungsbedingte Abnahme der Blattfläche bei geringer Belastung deutlich geringer darstellte als bei den anderen Belastungsstufen, war auch die BFD bei dieser Variante am höchsten. Die BFD bei $\mathbf{0 t}$ 2x5t und 6x5t lag auf dem gleichen Niveau aber $20 \%$ unter der BFD von $2 \times 2,5 t$.

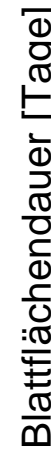

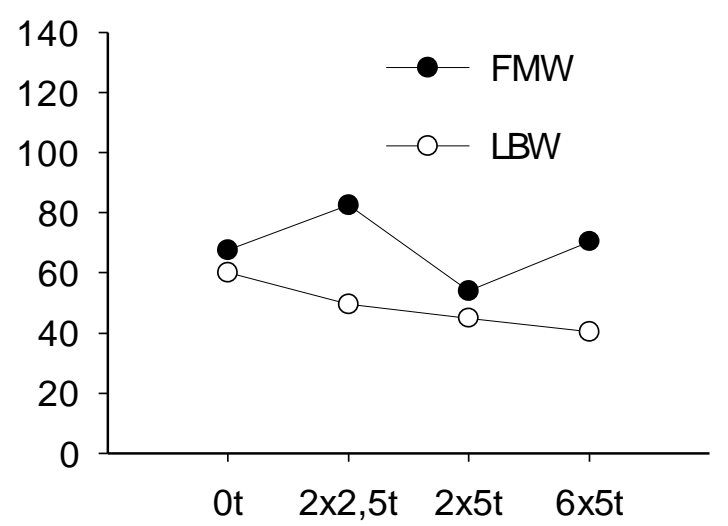

Abb. 33: Blattflächendauer (BFD) des Winterweizens vom Ende der Blüte (EC 69) bis zur mittleren Milchreife (EC 75) auf dem Standort Relliehausen 1996 in Abhängigkeit von der Belastungsstufe.

Überrollhäufigkeit x Radlast 
Bei einem Vergleich der beiden Bearbeitungssysteme zeigt sich bei FMW eine um ca. 45\% höhere BFD: Von EC 69 bis EC 75 stand auf dem flach bearbeiteten und gemulchten Boden wesentlich mehr Blattfläche zur Trockenmassebildung zur Verfügung als auf den gepflügten Varianten. Das war genau umgekehrt zu Reinshof (Abb. 26).

Abb. 34 zeigt die Bestandeshöhe und ihren Verlauf vom Beginn des Schossens bis zum Beginn der Blüte. Auf beiden Bearbeitungssystemen gab es keine signifikanten Unterschiede in der Bestandeshöhe. Tendenziell lagen die Werte für LBW 6x5t und FMW 6x5t zu EC 58 unter den Werten der anderen Belastungsstufen.
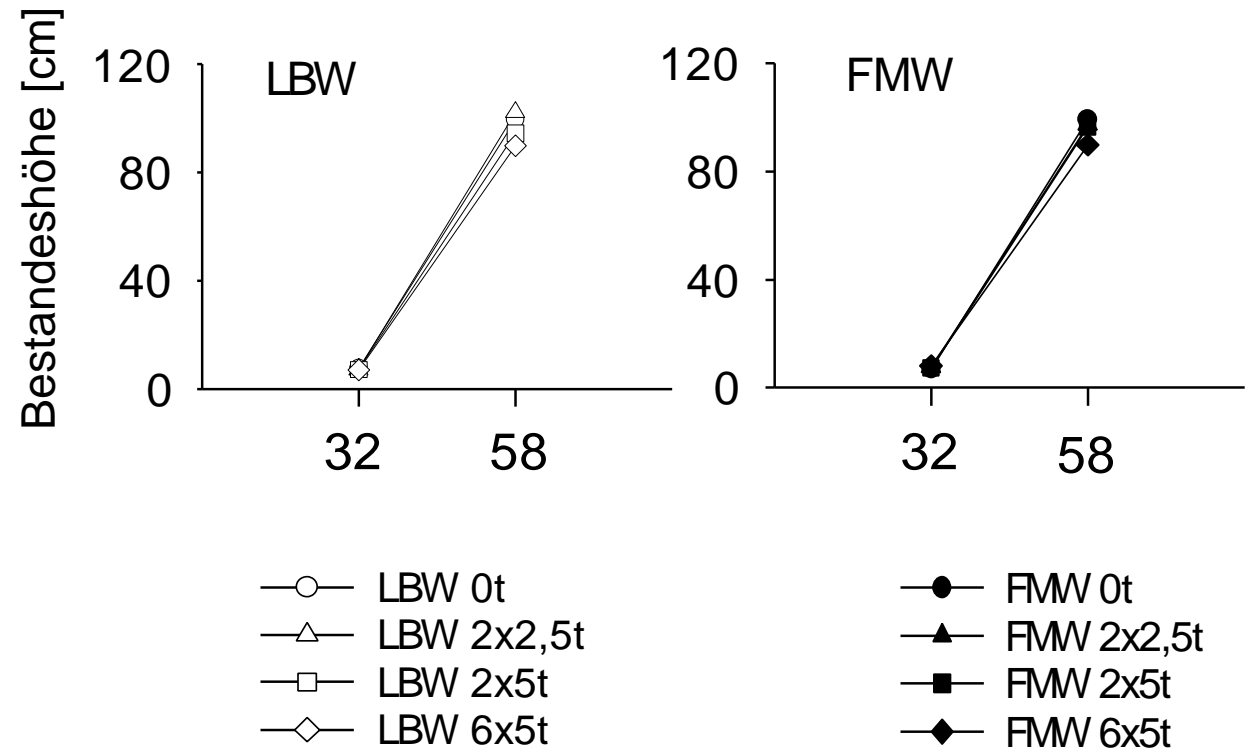

Abb. 34: Bestandeshöhe des Winterweizens auf dem Standort Relliehausen 1996 zu den Entwicklungsstadien EC 32 und EC 58.

Im dritten Versuchsjahr 1997 mußte in Relliehausen auf die Erfassung der Trockenmasse und der Blattfläche der Wintergerste aus arbeitstechnischen Gründen verzichtet werden. 


\subsubsection{Standort Großobringen}

\subsection{Versuchsjahr 1995}

Auf dem Standort Großobringen wurden 1995 keine Untersuchungen zum Trokkengewicht und zur Blattfläche der Sommergerste durchgeführt. Wohl aber wurde die Bestandeshöhe gemessen (Tab. 23). Tendenziell nahm mit steigender Bodenbelastung die Bestandeshöhe auf beiden Bearbeitungssystemen ab.

Tab. 23: Bestandeshöhe der Sommergerste zum Zeitpunkt der Ernte im Versuchsjahr 1995 auf dem Standort Großobringen. Gezeigt werden arithmetische Mittelwerte und die Grenzwerte des 95\%-Vertrauensintervalls.

\begin{tabular}{|c|c|c|c|c|}
\hline \multirow{3}{*}{ Bearbeitungssstem } & \multicolumn{4}{|c|}{ Bestandeshöhe [cm] } \\
\hline & \multicolumn{4}{|c|}{ Belastungsstufe } \\
\hline & $0 t$ & $2 \times 2,5 t$ & $2 \times 5 t$ & $6 \times 5 t$ \\
\hline LBW tief & $59( \pm 3,7)$ & $58( \pm 2,5)$ & $49( \pm 4,5)$ & $39( \pm 2,1)$ \\
\hline LBW flach & $58( \pm 1,2)$ & $56( \pm 2,2)$ & $45( \pm 4,0)$ & $41( \pm 4,0)$ \\
\hline
\end{tabular}

\subsection{Versuchsjahr 1996}

Im Versuchsjahr 1996 zeigte die Trockenmasse und die Blattfläche keine kennzeichnenden Unterschiede zwischen den Belastungsstufen bei den Systemen LBW tief und LBW flach (Abb. 35). Zwischen den Bearbeitungssystemen traten geringe Unterschiede auf. Auf dem tief gepflügten Boden (Abb. 35 links) lag die maximal ausgebildete Blattfläche geringfügig höher als auf dem flach gepflügten Boden (Abb. 35 rechts). Da die Blattfläche ansonsten keine auffallenden Unterschiede zwischen den Bearbeitungssystemen oder den Belastungsstufen aufweist, wurde auf eine Darstellung der BFD verzichtet. Um den Einfluß der mechanischen Bodenbelastung auf das Längenwachstum des Winterweizens im Versuchsjahr 1996 zu erkennen, wurde die Bestandeshöhe zur Zeit der Reife (EC 83) erfaßt (Tab. 24). Innerhalb der Bearbeitungssysteme konnte zwischen den Belastungsstufen kein Unterschied in der Bestandeshöhe festgestellt werden. Zwischen den Bearbeitungssystemen deuten die Werte auf eine reduzierte Bestandeshöhe bei LBW flach hin. 

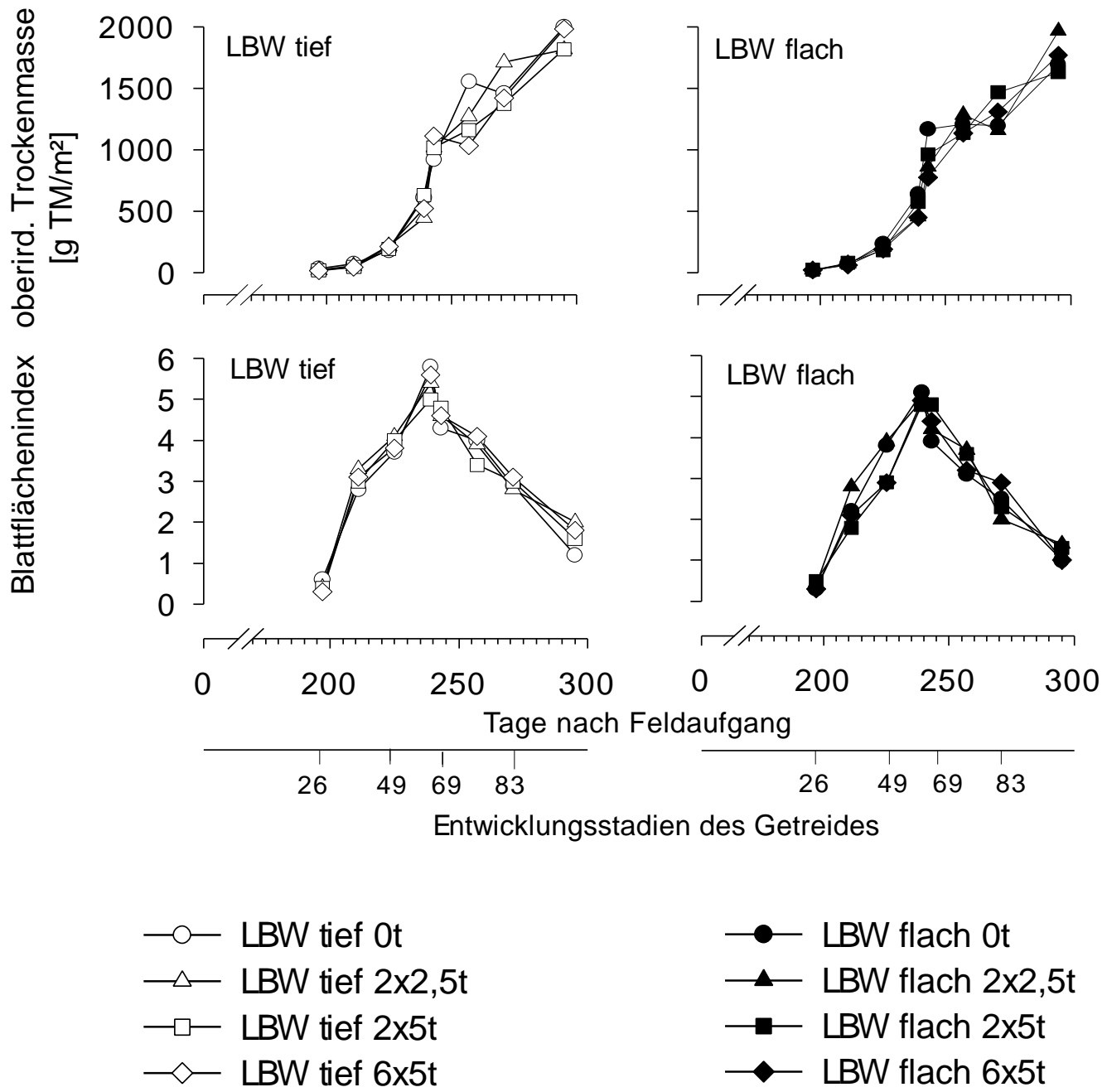

Abb. 35: Entwicklung der oberirdischen Trockenmasse (oben) und des Blattlächenindexes (unten) von Winterweizen über die Zeit auf dem Standort Großobringen im Versuchsjahr 1996.

Tab. 24: Bestandeshöhe des Winterweizens zum Zeitpunkt der Ernte im Versuchsjahr 1996 auf dem Standort Großobringen. Gezeigt werden arithmetische Mittelwerte und die Grenzwerte des 95\%-Vertrauensintervalls.

\begin{tabular}{c|cccc} 
& \multicolumn{4}{|c}{ Bestandeshöhe [cm] } \\
\hline Bearbeitungssystem & \multicolumn{4}{|c}{ Belastungsstufe } \\
& $\mathbf{0 t}$ & $\mathbf{2 \times 2 , 5 t}$ & $\mathbf{2 \times 5 t}$ & $\mathbf{6 x 5 t}$ \\
LBW tief & $95( \pm 2,8)$ & $92( \pm 3,1)$ & $96( \pm 2,0)$ & $96( \pm 2,3)$ \\
LBW flach & $88( \pm 2,5)$ & $88( \pm 1,5)$ & $90( \pm 3,1)$ & $89( \pm 2,0)$
\end{tabular}




\subsection{Versuchsjahr 1997}

Im dritten Versuchsjahr zeigte das Trockengewicht und die Blattfläche der Wintergerste auf dem tief gepflügten Boden bei $\mathbf{0 t}$, 2x2,5t und 6x5t keine deutlichen Unterschiede (Abb. 36 links). Bei 2x5t wurde zu Beginn des Schossens ein reduziertes Blattwachstum festgestellt. Auf dieser Variante erreichte der Bestand erst 214 Tage nach Feldaufgang einen BFI über 3. Bei den anderen Belastungsstufen wurde dieser Wert bereits 201 Tage nach Feldaufgang erreicht (Termin des Feldaufgangs siehe Tab. 59). Wahrscheinlich beeinträchtigte die geringe Blattfläche bei LBW tief 2x5t die Ausnutzung der eingestrahlten Energie und führte so zu einer verminderten Trockenmasseproduktion.
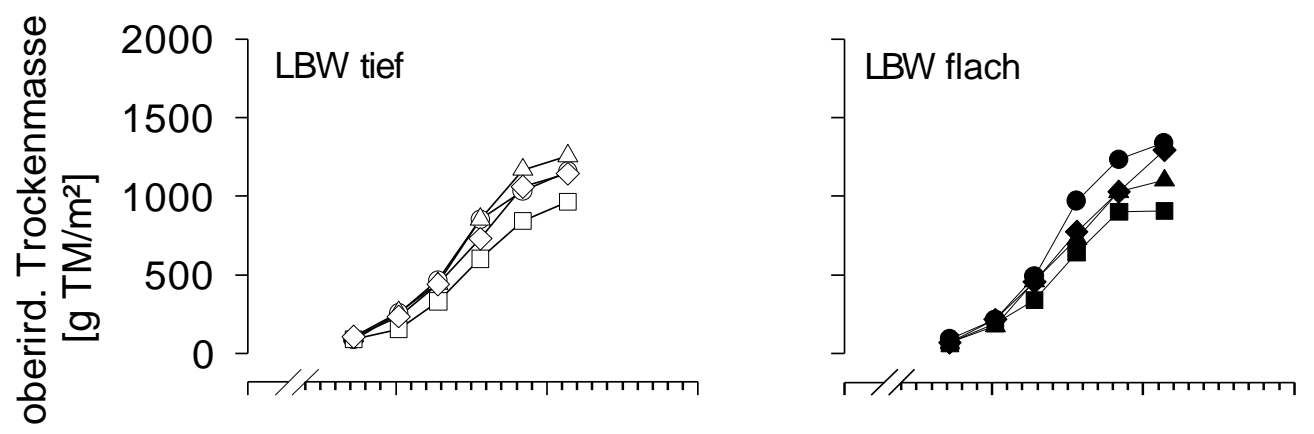

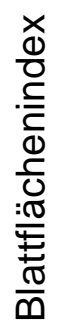
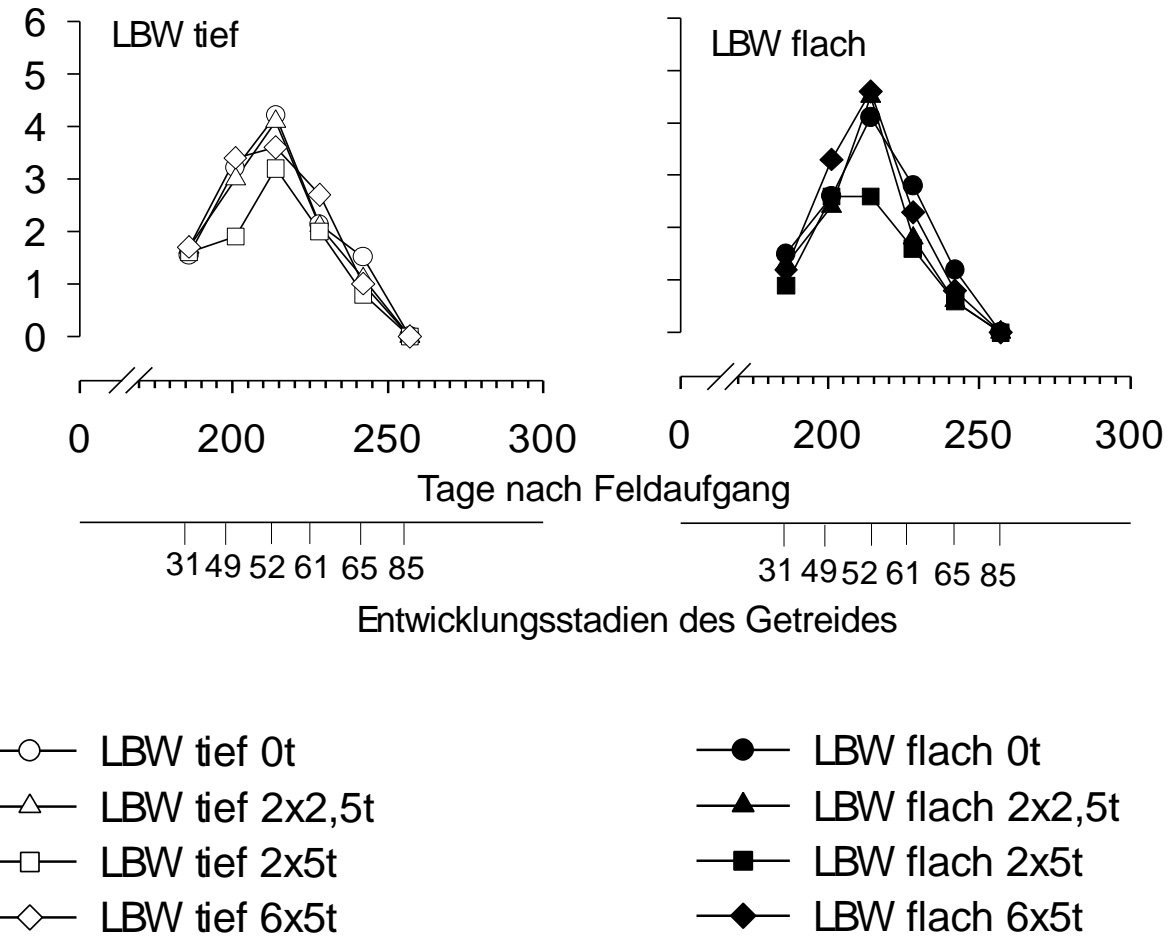

Tage nach Feldaufgang

Entwicklungsstadien des Getreides

$$
\begin{aligned}
& \multimap-\text { LBW tief } 0 \mathrm{t} \\
& \neg-\text { LBW tief } 2 \times 2,5 \mathrm{t} \\
& \square-\text { LBW tief } 2 \times 5 \mathrm{t} \\
& \checkmark-\text { LBW tief } 6 \times 5 \mathrm{t}
\end{aligned}
$$

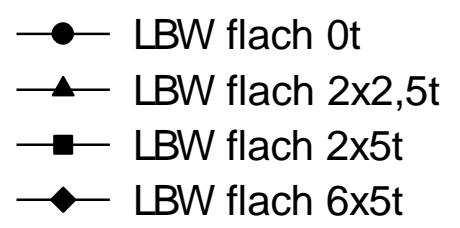

Abb. 36: Entwicklung der oberirdischen Trockenmasse (oben) und des Blattflächenindexes (unten) von Wintergerste über die Zeit auf dem Standort Großobringen im Versuchsjahr 1997. 
Auf dem flach gepflügten Boden (Abb. 36 rechts) zeigte das Trockengewicht und die Blattfläche keine deutlichen Unterschiede bei 0t, 2x2,5t und 6x5t. Bei 2x5t brach die Zunahme an Blattläche nach dem Schossen ab. Der BFI blieb bis zum Ährenschieben bei einem Wert von 2,5 konstant. Ab der Blüte nahm die Blattfläche bei allen Belastungsstufen ab. Die stark eingeschränkte Blattflächenentwicklung bei LBW flach $2 \times 5$ t hatte ähnlich wie bei LBW tief $2 \times 5$ t eine deutlich verminderte Trockenmassebildung zur Folge. Ein Einfluß der Spatenpflugtiefe auf das Trockengewicht und die Blattfläche war nicht festzustellen.

Aus Tab. 25 geht hervor, daß im letzten Versuchsjahr die Bestandeshöhe der Wintergerste bei beiden Bearbeitungssystemen und steigender Bodenbelastung verringert wurde. Bei einem Vergleich der Bearbeitungssysteme zeigte sich, daß die Bestandeshöhe auf dem flach gepflügten Boden deutlich unter der Bestandeshöhe auf dem tief gepflügten Boden lag. Bei LBW tief 2x5t lag die Bestandeshöhe bei beiden Bearbeitungssystemen auffällig niedrig und zeigte damit $\mathrm{Pa}$ rallelen zur Entwicklung von Trockenmasse und Blattfläche (siehe oben).

Tab. 25: Bestandeshöhe der Wintergerste zum Zeitpunkt der Ernte im Versuchsjahr 1997 auf dem Standort Großobringen. Gezeigt werden arithmetische Mittelwerte und die Grenzwerte des 95\%-Vertrauensintervalls.

\begin{tabular}{c|cccc} 
& \multicolumn{4}{|c}{ Bestandeshöhe [cm] } \\
\hline Bearbeitungssystem & \multicolumn{4}{|c}{ Belastungsstufe } \\
& $\mathbf{0 t}$ & $\mathbf{2 \times 2 , 5 t}$ & $\mathbf{2 \times 5 t}$ & $\mathbf{6 x 5 t}$ \\
LBW tief & $80( \pm 2,2)$ & $73( \pm 3,3)$ & $57( \pm 3,2)$ & $61( \pm 2,6)$ \\
LBW flach & $69( \pm 2,4)$ & $62( \pm 4,3)$ & $57( \pm 1,9)$ & $55( \pm 2,4)$
\end{tabular}




\subsubsection{Gaswechsel und Gesamtwasserpotential}

Wasser ist von außerordentlich großer Bedeutung für das Leben der Pflanzen. Das über die Wurzeln aufgenommene Bodenwasser gelangt durch den Sproß ins Blatt. Von der Blattoberfläche wird es als Wasserdampf an die Atmosphäre abgegeben. Die Menge des aufgenommenen Bodenwassers und die Menge des durch Transpiration abgegebenen Wassers beeinflussen das Gesamtwasserpotential in der Pflanze. Die Höhe des Gesamtwasserpotentials beeinflußt viele physiologische Prozesse und kann ein Maß für Wasserstreß in der Pflanze darstellen.

Neben der photosynthetisch aktiven Strahlung (PAR) ist das Vorhandensein von $\mathrm{CO}_{2}$ in den Chloroplasten eine Voraussetzung für die photosynthetischen Stoffwechselprozesse einer Pflanze. $\mathrm{CO}_{2}$ dient als Kohlenstoffquelle zur Biosynthese von Kohlehydraten und damit zur Substanzproduktion. Die $\mathrm{CO}_{2}-$ Aufnahme in das Blatt geschieht - ebenso wie die Transpiration von Wasserdampf - über die Stomata. Treibender Motor für beide Prozesse der Aufnahme und der Abgabe ist die Diffusion entlang eines Konzentrationsgefälles. Für die Abgabe des Wasserdampfes gibt das Sättigungsdefizit der umgebenden Luft einen Hinweis auf die Größe des Konzentrationsgefälles. Regelmechanismen steuern die Raten des Gaswechsels über die Öffnungsweite der Stomata. Als Maß für den Öffnungszustand der Stomata dient die stomatäre Leitfähigkeit. $\mathrm{CO}_{2}-$ Assimilation, Transpiration und Gesamtwasserpotential sind eng über die Regelmechanismen der Stomata miteinander verbunden.

Am 23.06.1996 wurden auf dem Standort Reinshof Messungen zum Gaswechsel des Winterweizens durchgeführt. Abb. 37 faßt mit der PAR und dem Sättigungsdefizit der Luft Witterungsbedingungen zur Zeit der Messung zusammen. Dargestellt ist in Abb. 38 die $\mathrm{CO}_{2}$-Assimilation und die stomatäre Leitfähigkeit des Winterweizens, in Abb.39 das zeitgleich gemessene Gesamtwasserpotential der Pflanzen und in Abb. 40 der Wassergehalt des Bodens zum Termin der Gaswechselmessung. 


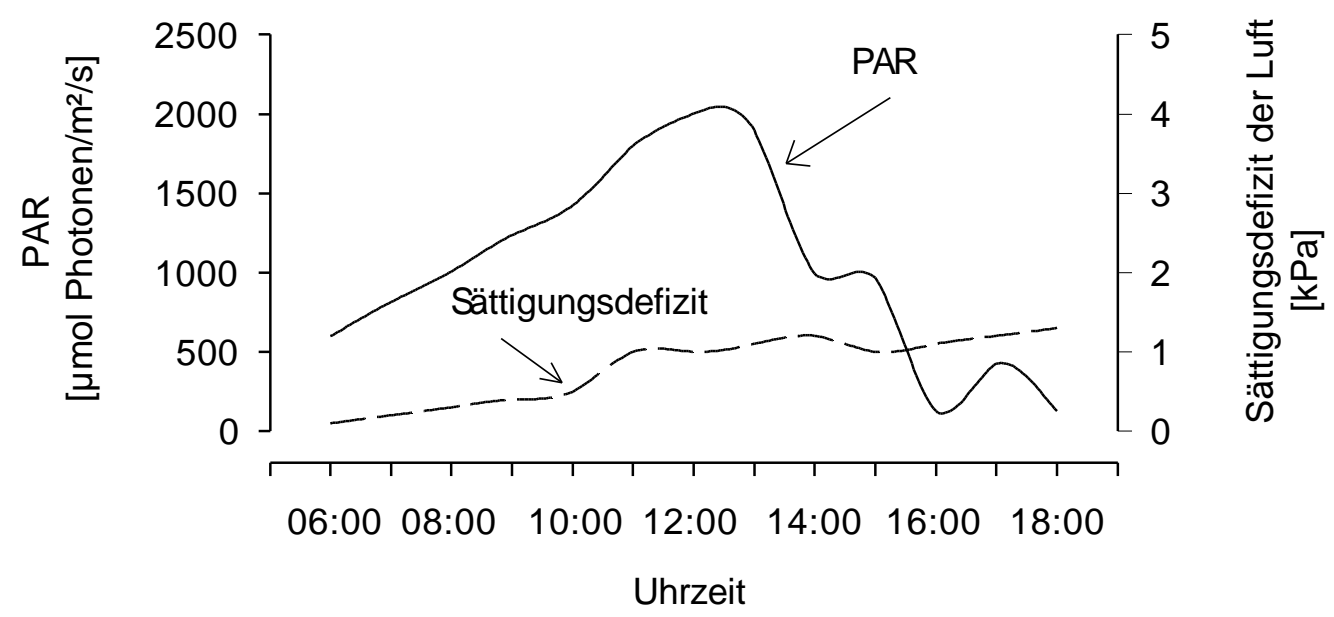

Abb. 37: Gang der photosynthetisch aktiven Strahlung (PAR) und des Sättigungsdefizites am 23.06.1996 auf dem Standort Reinshof.

LBW

FMW

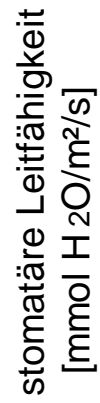

$\left.\begin{array}{c}800 \\ 600 \\ 400 \\ 200 \\ 0\end{array}\right]$
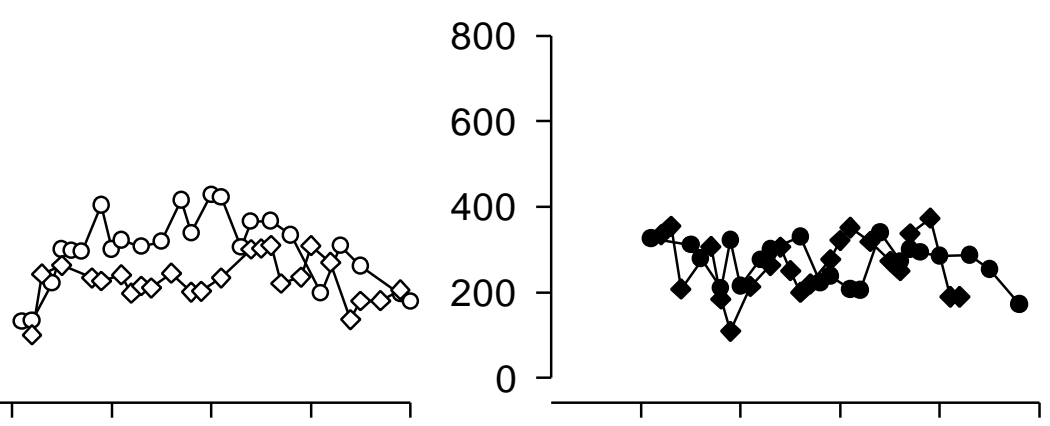

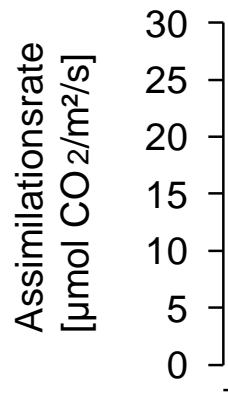
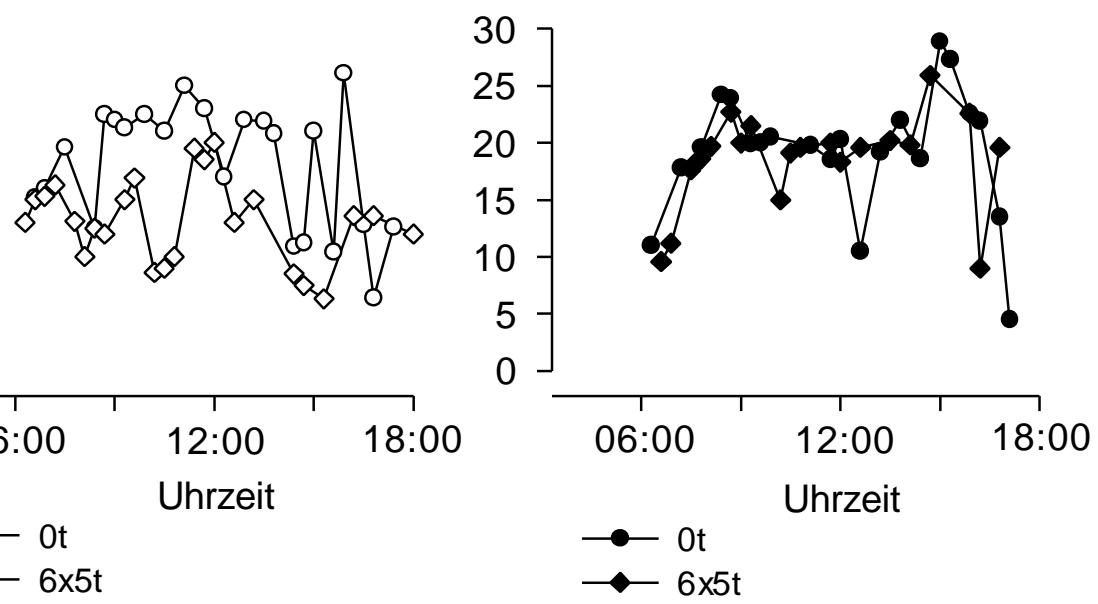

Abb. 38: Stomatäre Leittähigkeit (oben) und $\mathrm{CO}_{2}$-Assimilationsrate des Winterweizens (unten) gemessen am Fahnenblatt auf dem Standort Reinshof als Funktion der Zeit am 23.06.1996 zum Stadium EC 69. 
LBW

FMW

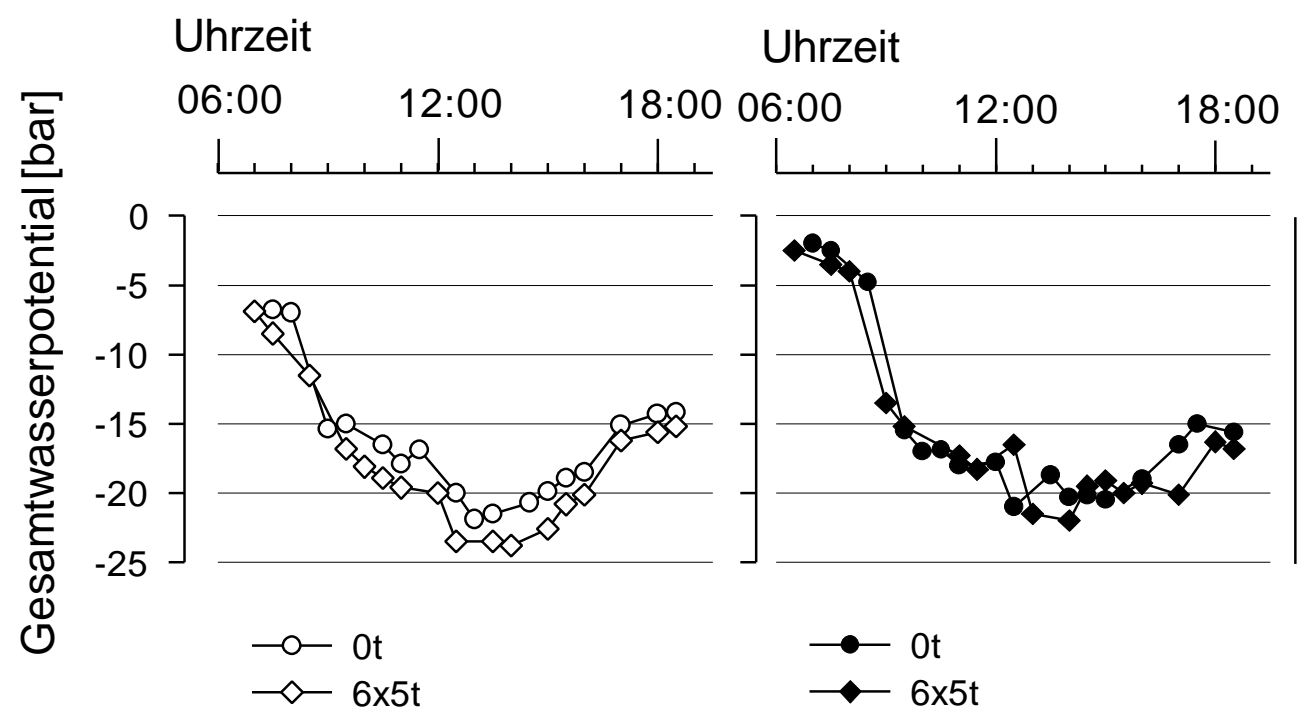

Abb. 39: Gesamtwasserpotential im Winterweizen als Funktion der Zeit am 23.06.1996 auf dem Standort Reinshof zum Stadium EC 69.

LBW

FMW

Bodenwassergehalt [Vol.\%] Bodenwassergehalt [Vol.\%]

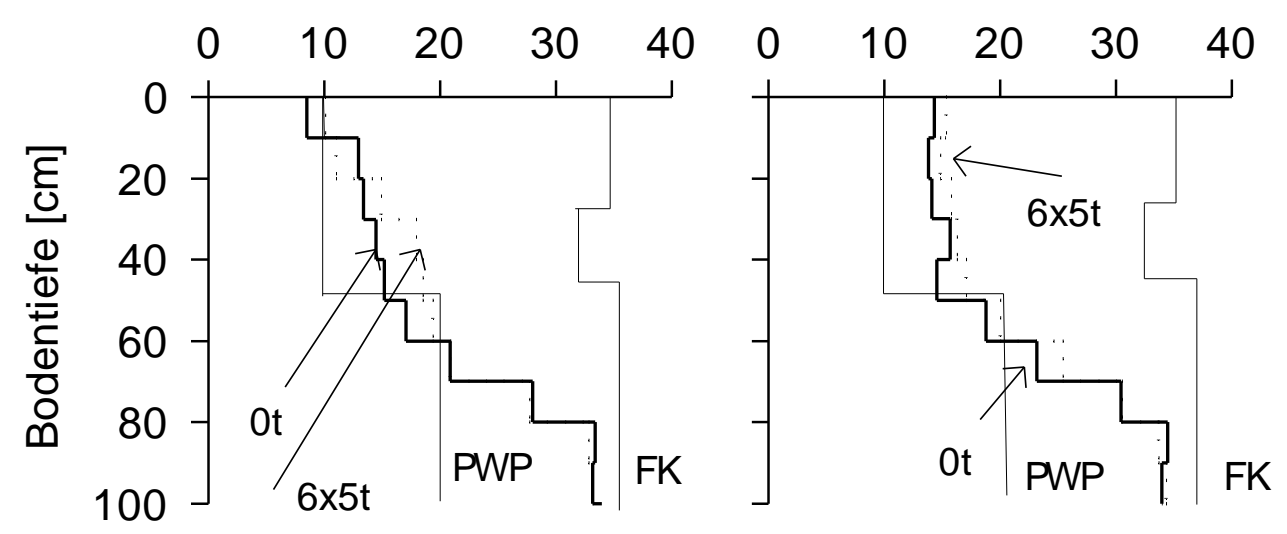

Abb. 40: Bodenwassergehalt als Funktion der Bodentiefe auf dem Standort Reinshof am 23.06.1996. Die Feldkapazität (FK) und der Permanente Welkepunkt (PWP) sind nach Angaben von BORNSCHEUER (1984) zusammengestellt. 
Wie Abb. 37 zeigt, nahm die PAR am 23.06.1996 von 06:00 Uhr bis ca. 12:00 Uhr stark zu. Höchstwerte wurden zwischen 11:00 und 12:00 Uhr mit ca. 2000 $\mu \mathrm{mol}$ Photonen $/ \mathrm{m}^{2} / \mathrm{s}$ erreicht. Von 12:00 bis 18:00 Uhr nahm die PAR bis auf Werte unter $500 \mu \mathrm{mol}$ Photonen/m²/s ab. Die Abnahme der PAR war die Folge einer aufziehenden dichten Bewölkung. Das Sättigungsdefizit der Luft lag um 06:00 Uhr knapp über null. Über den Tagesgang nahm das Sättigungsdefizit der Luft bis auf 1,3 kPa zu. Insgesamt lagen die Werte auf einem sehr niedrigen Niveau. An strahlungsreichen Tagen können Werte bis zu 4 kPa erreicht werden (EHLERS 1996).

Bei LBW lag die stomatäre Leitfähigkeit des Weizens bei hoher Bodenbelastung fast über den gesamten Tag unter der Leitfähigkeit des Weizens auf dem Boden ohne Belastung (Abb. 38 links oben). Bei geringer und mittlerer Belastung (nicht dargestellt) lagen die Meßwerte annähernd auf der Höhe der Kontrolle (LBW 0t). Offensichtlich war die Öffnungsweite der Stomata bei Ot über den Tag größer als bei 6x5t. Eine Ausnahme bildete der Zeitabschnitt von 06:00 bis 07:00 Uhr: Zu Anfang des Tages unterschieden sich die Öffnungsweiten der Stomata nicht. Wie Abb. 38 (links unten) zeigt, war auch die $\mathrm{CO}_{2}{ }^{-}$ Assimilationsrate bei $0 t$ im Durchschnitt höher als bei 6x5t. Die Werte bei 2x2,5t und 2x5t (nicht dargestellt) lagen zwischen den Belastungsstufen 0t und 6x5t. Auf dem hoch belasteten Boden hatte die geringere Öffnungsweite der Stomata eine Einschränkung der $\mathrm{CO}_{2}$-Aufnahme zur Folge.

Der Tagesverlauf des Gesamtwasserpotentials (GWP) des Weizens im Tagesverlauf zeigte auf dem gepflügten Boden bemerkenswerte Unterschiede zwischen den Belastungsstufen 0t und 6x5t (Abb. 39 links): Bis ca. 09:00 Uhr fielen die Werte des GWP bei 0t und 6x5t von -7 bar auf -15 bar (bitte beachten: Fallende Werte der GWP bedeuten einen Anstieg des Wasserstresses in der Pflanze). Von etwa 09:00 bis 14:00 Uhr nahmen die Werte auf dem hoch belasteten Boden merklich stärker $\mathrm{ab}$ als auf dem nicht belasteten Boden - der Wasserstreß des Weizens auf der hoch belasteten Variante war deutlich höher als auf der Kontrolle (LBW Ot). Nach 14:00 Uhr stiegen die Werte des GWP bis zum Ende der Messung bei beiden Varianten wieder an, der Wasserstreß nahm ab. Wie auch in der Zeit von 09:00 bis 14:00 Uhr unterlagen die Pflanzen auf dem hoch belasteten Boden von 14:00 bis 18:00 einem auffallend höheren Wasserstreß als die Pflanzen auf dem Boden ohne Belastung. Die Werte des 
GWP bei geringer und mittlerer Bodenbelastung (nicht dargestellt) entsprachen im Tagesverlauf in etwa der Kontrolle (LBW 0t). Aus Abb.40 geht der zeitgleich erfaßte Bodenwassergehalt als Funktion der Bodentiefe hervor: Auf dem gepflügten Boden unterschritt der Wassergehalt des Bodens bei $6 x 5 t$ den PWP in der obersten Bodenschicht $(0-10 \mathrm{~cm})$ und in der Bodenschicht $50-60 \mathrm{~cm}$ geringfügig, pflanzenverfügbares Wasser stand nicht mehr zur Verfügung (Abb. 40 links). Mit zunehmender Bodentiefe nahm bei beiden Belastungsstufen der Bodenwassergehalt zu. Von $20 \mathrm{~cm}$ bis $60 \mathrm{~cm}$ Bodentiefe lag der Wassergehalt auf dem hoch belasteten Boden um 1-2 Vol. \% über dem Gehalt an Bodenwasser auf dem nicht belasteten Boden. Unterhalb von $60 \mathrm{~cm}$ Bodentiefe war der Wassergehalt bei $0 t$ und $6 \times 5 t$ identisch.

Bei FMW zeigte die stomatäre Leitfähigkeit des Winterweizens (Abb. 38 rechts oben) auf dem Boden ohne, mit geringer (nicht dargestellt), mit mittlerer (nicht dargestellt) und schließlich mit hoher Bodenbelastung keine kennzeichnenden Unterschiede. So wie die stomatäre Leitfähigkeit, so stellte sich auch die Assimilationsrate dar (2x2,5t und $\mathbf{2 \times 5 t}$ sind nicht dargestellt): Über den Zeitabschnitt von 06:00 bis 18:00 Uhr wich die Assimilationsrate der Pflanzen auf dem hoch belasteten Boden nicht auffallend von der Kontrolle (FMW 0t) ab (Abb. 38 rechts unten). Wie Abb.39 rechts zeigt, wies auch das GWP im Tagesgang keine deutlichen Unterschiede zwischen den Belastungsstufen bei FMW auf. Die Werte bei $\mathbf{2 x 2 , 5 t}$ und 2x5t (nicht dargestellt) entsprachen in etwa der Kontrolle (FMW 0t).

Bei einem Vergleich des GWP bei LBW und FMW zeigte sich auffallende Unterschiede: Auf dem gepflügten Boden war das Potential zu Beginn der Messung um 06:00 Uhr erheblich niedriger als auf dem flach bearbeiteten und gemulchten Boden (Abb. 39 rechts). Offenbar konnten sich FMW - Pflanzen über Nacht besser erholen als die auf LBW. Bei LBW stellte sich über Mittag ein stärkerer Wasserstreß ein als bei FMW.

Der Bodenwassergehalt bei FMW lag bei allen Belastungsstufen $(2 \times 2,5 t$ und 2x5t sind nicht dargestellt) von der obersten Bodenschicht bis zu einer Bodentiefe von $50 \mathrm{~cm}$ bei ca. 15 Vol. \% (Abb. 40 rechts). Von $50 \mathrm{~cm}$ bis $80 \mathrm{~cm}$ Bodentiefe nahm der Gehalt an Bodenwasser bei allen Belastungsstufen bis auf 30 Vol.\% zu. In der obersten Bodenschicht bis ca. $20 \mathrm{~cm}$ Bodentiefe lag der 
Bodenwassergehalt auf dem flach bearbeiteten Boden deutlich über dem Wassergehalt des gepflügten Bodens.

Abb. 41 zeigt die assimilierte $\mathrm{CO}_{2}$ - Menge als Funktion der PAR für Winterweizen auf gepflügtem Boden mit den Belastungsstufen ot und 6x5t am 23.06.1996. Die Regressionskurve, die den Verlauf einer Hyperbel hat, zeigt die assimilierte $\mathrm{CO}_{2}$-Menge als Funktion vom Lichtfluß und wird fortan als LichtflußEffektkurve bezeichnet (MOHR \& SCHOPFER 1985). Ein Vergleich der LichtflußEffektkurven (Abb. 41) zeigt Unterschiede zwischen den Belastungsstufen 0t und 6x5t auf dem gepflügten Boden: Ab einem Lichtfluß von über $300 \mu \mathrm{mol}$ Photonen $/ \mathrm{m}^{2} / \mathrm{s}$ lag die Assimilationsrate der Pflanzen auf dem nicht belasteten Boden stets über der Assimilationsrate auf dem hoch belasteten Boden.

Um die Assimilationsraten aller Belastungsstufen auf dem gepflügten und dem flach bearbeiteten und gemulchten Boden vergleichen zu können, sind die Lichtfluß-Effektkurven in Abb.42 dargestellt.

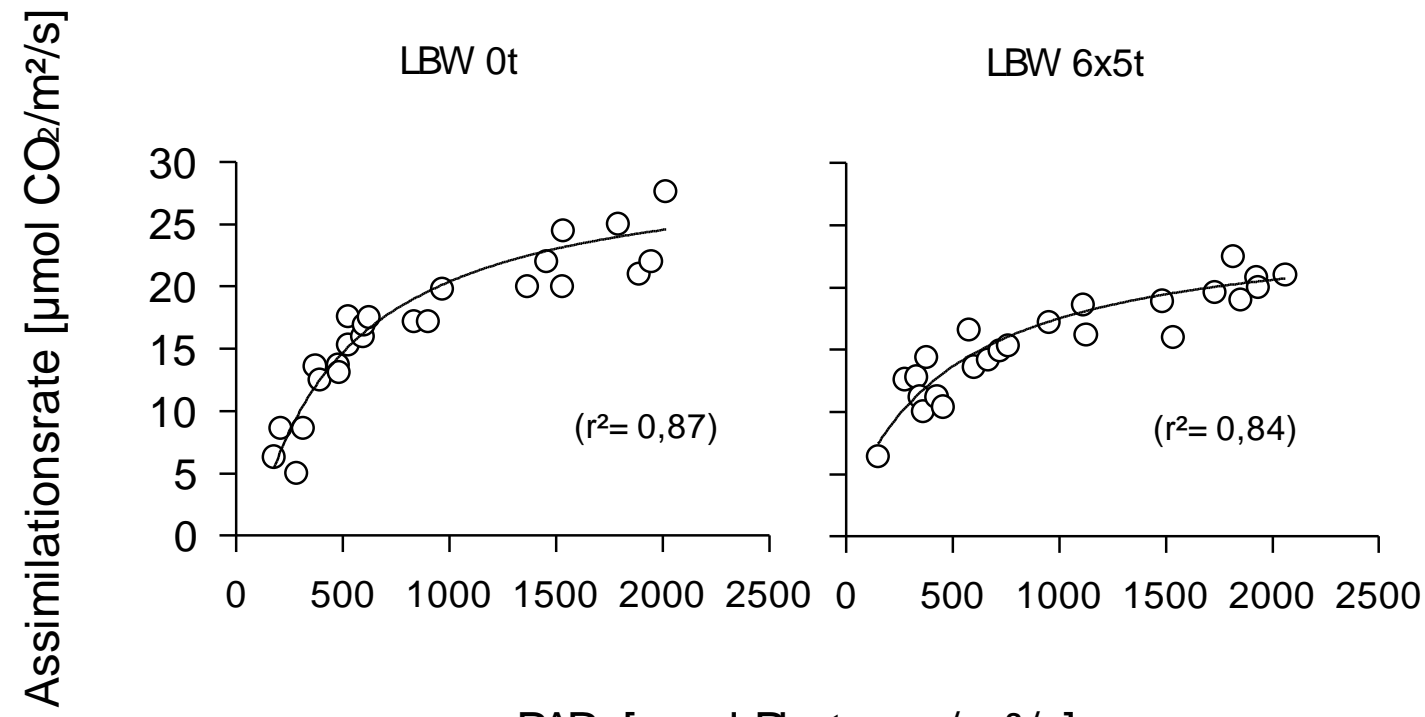

PAR $\left[\mu \mathrm{mol}\right.$ Photonen $\left./ \mathrm{m}^{2} / \mathrm{s}\right]$

Abb. 41: Die $\mathrm{CO}_{2}$-Assimilationsrate von Winterweizen (gemessen am Fahnenblatt) als Funktion der photosynthetisch aktiven Strahlung (PAR) zum Stadium EC 69 bei Lockerbodenwirtschaft (LBW) auf dem Standort Reinshof vom 23.06.1996. 


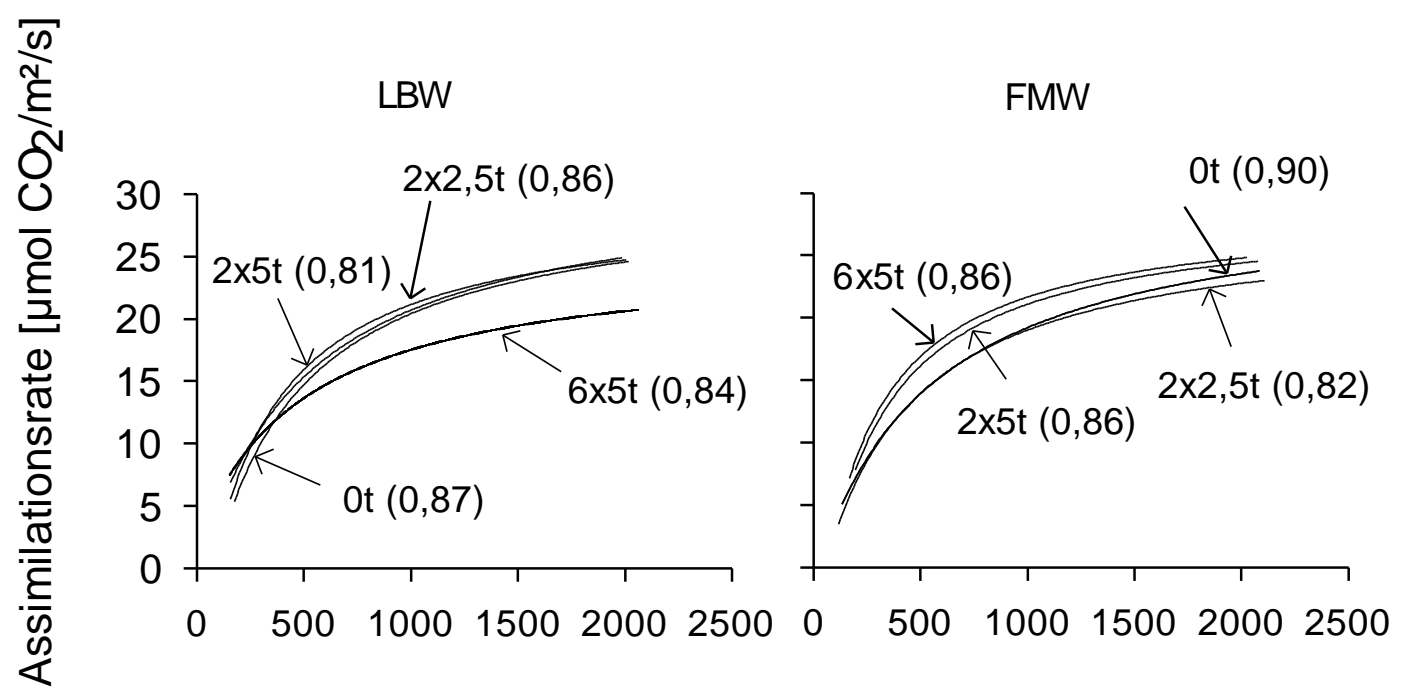

PAR $\left[\mu \mathrm{mol}\right.$ Photonen $\left./ \mathrm{m}^{2} / \mathrm{s}\right]$

Abb. 42: Lichtfluß-Effektkurven des Winterweizens (gemessen am Fahnenblatt) am 23.06.1996 im Stadium EC 69 auf dem Standort Reinshof. Die Zahlen in Klammern zeigen das Bestimmtheitsmaß $\left(r^{2}\right)$ der Regression.

Bei LBW 0t, 2x2,5t und 2x5t lagen die $\mathrm{CO}_{2}$-Assimilationsraten über den gesamten Bereich der eingestrahlten Lichtmenge auf dem gleichen Niveau (Abb. 42 links). Auf dem hoch belasteten Boden war bei geringer Lichtmenge $(<300$ umol Photonen $/ \mathrm{m}^{2} / \mathrm{s}$ ) die Assimilationsrate so hoch wie bei der Kontrolle (LBW 0t). Mit zunehmender Lichtmenge ( $>300 \mu \mathrm{mol}$ Photonen $/ \mathrm{m}^{2} / \mathrm{s}$ ) lagen die Werte der Assimilationsraten bei 6x5t deutlich unter der Kontrolle (LBW 0t). Die Differenz wurde mit zunehmendem Lichtfluß zwischen 6x5t und 0t größer: Bei 2000 $\mu \mathrm{mol}$ Photonen $/ \mathrm{m}^{2} / \mathrm{s}$ lag die Assimilationsrate von 6x5t um $19 \%$ unter den Werten bei 0t. Bei FMW (Abb. 42 rechts) zeigte sich ein ganz anderes Bild. Über den ganzen Bereich der eingestrahlten Lichtmenge lagen die Werte bei $\mathbf{0 t}$ und 2x2,5t auf einer Höhe. Ebenso unterschied sich die Assimilationsrate von $2 \times 5 t$ und $6 \times 5 t$ nur unwesentlich. Bemerkenswert ist das Verhältnis von Assimilationsrate und Belastungsstufe: Im Gegensatz zum gepflügten Boden lagen auf dem flach bearbeitetet und gemulchten Boden die Assimilationsraten auf dem Boden mit mittlerer und hoher Belastung über einen weiten Strahlungsbereich (350 - $1500 \mu \mathrm{mol}$ Photonen $/ \mathrm{m}^{2} / \mathrm{s}$ ) ca. 15\% über den Assimilationsraten auf dem nicht oder gering belasteten Boden. Im Vergleich der Bearbeitungssysteme zeigte sich ein annähernd identischer Verlauf der Lichtfluß-Effektkurven von 
LBW 0t und FMW 0t. Das Bearbeitungssystem hatte ohne zusätzliche Bodenbelastung zum Meßtermin keinen Einfluß auf die Höhe der $\mathrm{CO}_{2}$ Assimilationsrate der Kulturpflanzen als Funktion der PAR.

Im Jahr 1996 konnten die $\mathrm{CO}_{2} / \mathrm{H}_{2} \mathrm{O}$-Gaswechselmessungen nur mit sehr geringem Probenumfang durchgeführt werden, da die Eichung des Porometers groBe Schwierigkeiten machte und ein $\mathrm{CO}_{2}$ - Regelventil defekt war. Im Jahr 1997, dem letzten Versuchsjahr, konnten die technischen Mängel eliminiert werden, so daß umfangreichere Messungen möglich waren. Im Rahmen der Gaswechselmessungen waren Meßphasen bei hohem Wasserstreß in den Pflanzen von besonderem Interesse: Unter Streßbedingungen wurden die größten Unterschiede zwischen den Varianten erwartet. Um den Gaswechsel bei möglichst hohem Wasserstreß in den Pflanzen erfassen zu können, wurden vom 04.06.1997 bis zum 19.06.1997 Messungen am Fahnenblatt der Wintergerste durchgeführt. Im untersuchten Zeitabschnitt fiel kein Niederschlag, die Witterung konnte als sommerlich warm beschrieben werden. Ziel der Untersuchung war es, möglichst lange Tagesgänge bei wolkenfreiem Himmel mit hoher PAR und hohem Sättigungsdefizit zu erfassen. Werte vor und knapp nach Sonnenaufgang sollten Aufschluß geben über die Gaswechselvorgänge nahe des Lichtkompensationspunktes. Die Witterungsbedingungen im untersuchten Zeitabschnitt ermöglichten es nicht, einen kompletten Tagesgang von früh morgens bis zum späten Nachmittag zu erfassen. Aufkommende Bewölkung führte an den Meßtagen zu unterschiedlichen Tageszeiten zu einem Abbruch der Messungen. Um dennoch einen Tagesgang über einen möglichst langen Zeitabschnitt betrachten zu können, wurden in den Abb. 43-45 die Messungen des 18.06.1997 (von 04:10 bis 08:00) und des 19.06.1997 (von 08:00 bis 15:40) zusammengefaßt. Zu diesen Zeiten wurde die Sonneneinstrahlung bis 09:00 Uhr nicht durch sichtbare Wolkenbildung abgeschirmt (Abb. 43). Zwischen 9:30 und 13:30 Uhr bildete sich eine leichte Wolkendecke, die PAR nahm ab. Von ca. 13:30 Uhr bis 14:30 Uhr war der Himmel wolkenfrei. Danach kam es zu einer schnell zunehmenden dichten Bewölkung, so daß die Messung um etwa 15:30 Uhr abgebrochen werden mußte. Das Sättigungsdefizit der Luft stieg von etwa $0 \mathrm{kPa}$ bis auf ca. 1,3 kPa um 11:00 Uhr an. Von 11:00 bis ca. 12:00 Uhr blieb das Sättigungsdefizit auf diesem Niveau. Um 14:00 Uhr - bei zunehmen- 
der PAR - stiegen die Werte auf knapp $2 \mathrm{kPa}$ an. So wie die PAR, so nahm auch das Sättigungsdefizit bei zunehmender Bewölkung nach 14:30 Uhr ab.

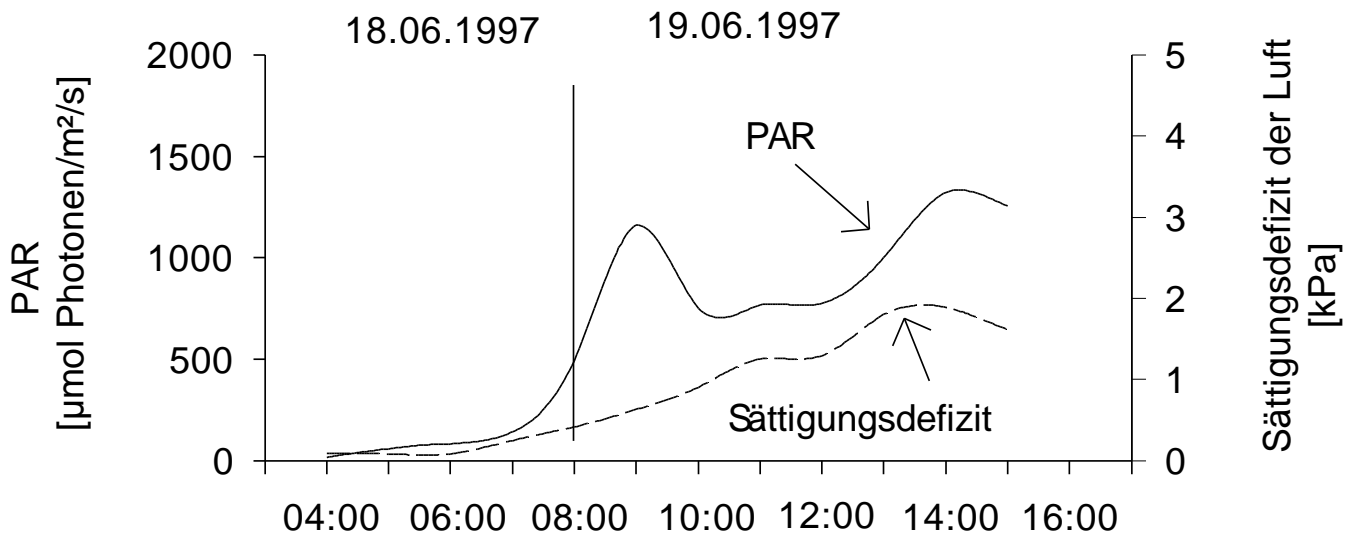

Uhrzeit

Abb. 43: Gang der photosynthetisch aktiven Strahlung (PAR) und des Sättigungsdefizites am 18.06.1997 und am 19.06.1997 auf dem Standort Reinshof.

Bei LBW (Abb. 44 links) bestätigten die Beobachtungen des Tagesganges die Erfahrungen vom Vorjahr mit Winterweizen: Bei hoher Belastung lag die stomatäre Leitfähigkeit der Wintergerste, ebenso wie die $\mathrm{CO}_{2}$-Assimilationsrate tendenziell unter den Werten bei 0t. Auf dem hoch belasteten Boden führte ein stärkerer Spaltenschluß der Stomata zu einer Beeinträchtigung der $\mathrm{CO}_{2}^{-}$ Assimilationsrate. Die Werte der stomatären Leitfähigkeit und der $\mathrm{CO}_{2}{ }^{-}$ Assimilationsrate auf dem Boden mit geringer und mittlerer Belastung (nicht dargestellt) entsprachen in etwa den Werten der Kontrolle (LBW Ot). Wie aus Abb.45 (oben) hervorgeht, war zur selben Zeit bei LBW 6x5t der Wasserstreß in den Pflanzen auffallend höher als bei der Kontrolle. Die Pflanzen bei 6x5t unterlagen in der Regel einem höheren Wasserstreß. Zur Mittagszeit wurden Spitzenwerte des Gesamtwasserpotentials von -25 bar erreicht.

Eine Betrachtung der Ausschöpfung des Bodenwassers vom 04.06 bis zum 19.06.1997 in Abb.46 (links) zeigte einen ungleichen Wasserentzug bei LBW 0t und LBW 6x5t. In der Bodenschicht von 10-60 cm lag die Menge des aufgenommenen Wassers bei 6x5t deutlich über dem Entzug bei $0 t$. Unterhalb von $60 \mathrm{~cm}$ nahmen die Pflanzen bei 6x5t in der beobachteten Zeit kein Bodenwasser auf. Bei den Pflanzen auf der Kontrolle war ein Wasserentzug bis in eine Bodentiefe von $90 \mathrm{~cm}$ festzustellen. Der starke Wasserentzug aus den oberen 
Bodenschichten bei 6x5t führte zu einer kompletten Entleerung des Vorrats an pflanzenverfügbaren Wassers in der Bodenschicht 0-20 cm (nicht dargestellt). Bei 0t wurde der permanente Welkepunkt (PWP) nur in der Bodenschicht 0-10 cm erreicht. Insgesamt entzogen die Pflanzen vom 04.06. bis zum 19.06.1997 bei 6x5t $17 \%$ mehr Bodenwasser als die Pflanzen auf der Kontrolle (LBW 0t). Bei FMW (Abb. 44 rechts) stellte sich eine andere Entwicklung dar: Die stomatäre Leitfähigkeit und die $\mathrm{CO}_{2}$-Assimilationsrate nahm mit steigender Bodenbelastung zu (2x2,5t und $2 \times 5 t$ sind nicht dargestellt). Dies gilt besonders für die Zeit von 09:00 Uhr bis 15:00 Uhr. Eine Betrachtung des Gesamtwasserpotentials (Abb. 45 unten) zeigte bei FMW (2×2,5t und $2 \times 5$ t sind nicht dargestellt) keine Unterschiede zwischen den Belastungsstufen. Anders als bei LBW lag bei FMW die Bodenwasserausschöpfung in der Bodenschicht 10-30 $\mathrm{cm}$ bei 0t über dem Entzug bei 6x5t (Abb. 46 rechts). In der Bodenschicht 0-10 cm und unter $30 \mathrm{~cm}$ Bodentiefe war die Wasseraufnahme von $0 t$ und $6 \times 5 \mathrm{t}$ annähernd gleich. Insgesamt lag der Wasserentzug in der beobachteten Zeit bei 0 t $17 \%$ über dem Entzug von $6 \times 5 t$. 
LBW
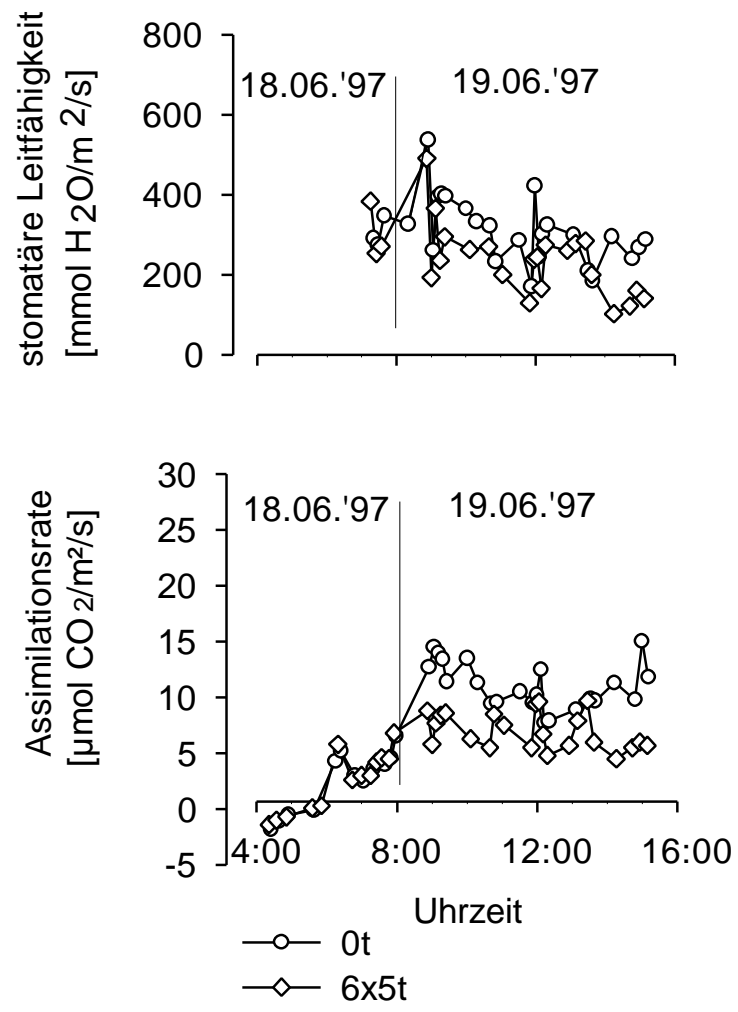

FMW
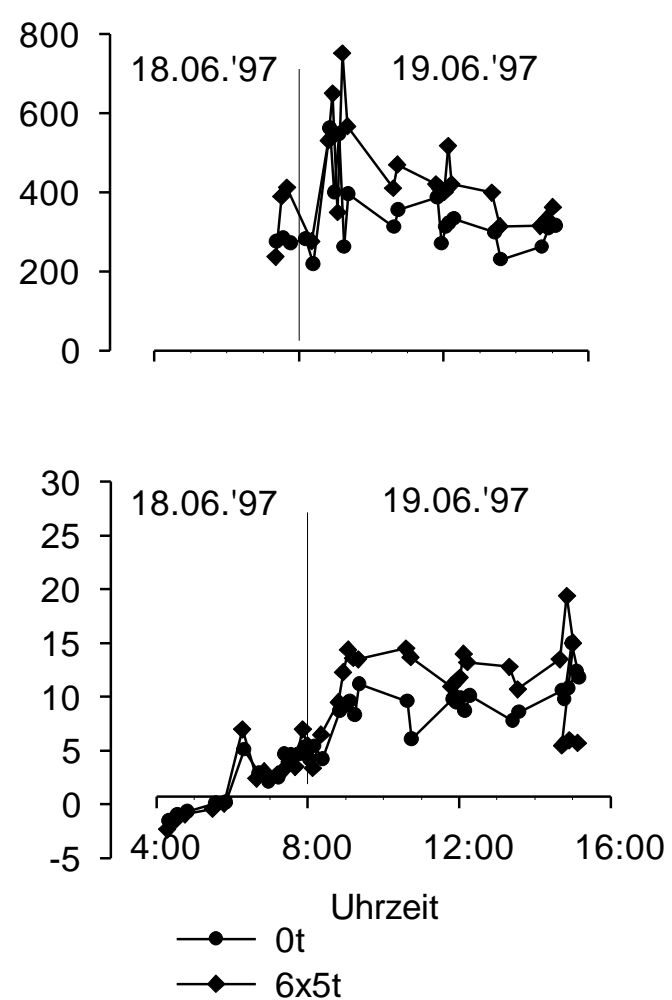

Abb. 44: Stomatäre Leitfähigkeit (oben) und $\mathrm{CO}_{2}$-Assimilationsrate des Wintergerste (unten) gemessen am Fahnenblatt auf dem Standort Reinshof als Funktion der Zeit am 18. und 19. 06.1997 zum Stadium EC 83. 


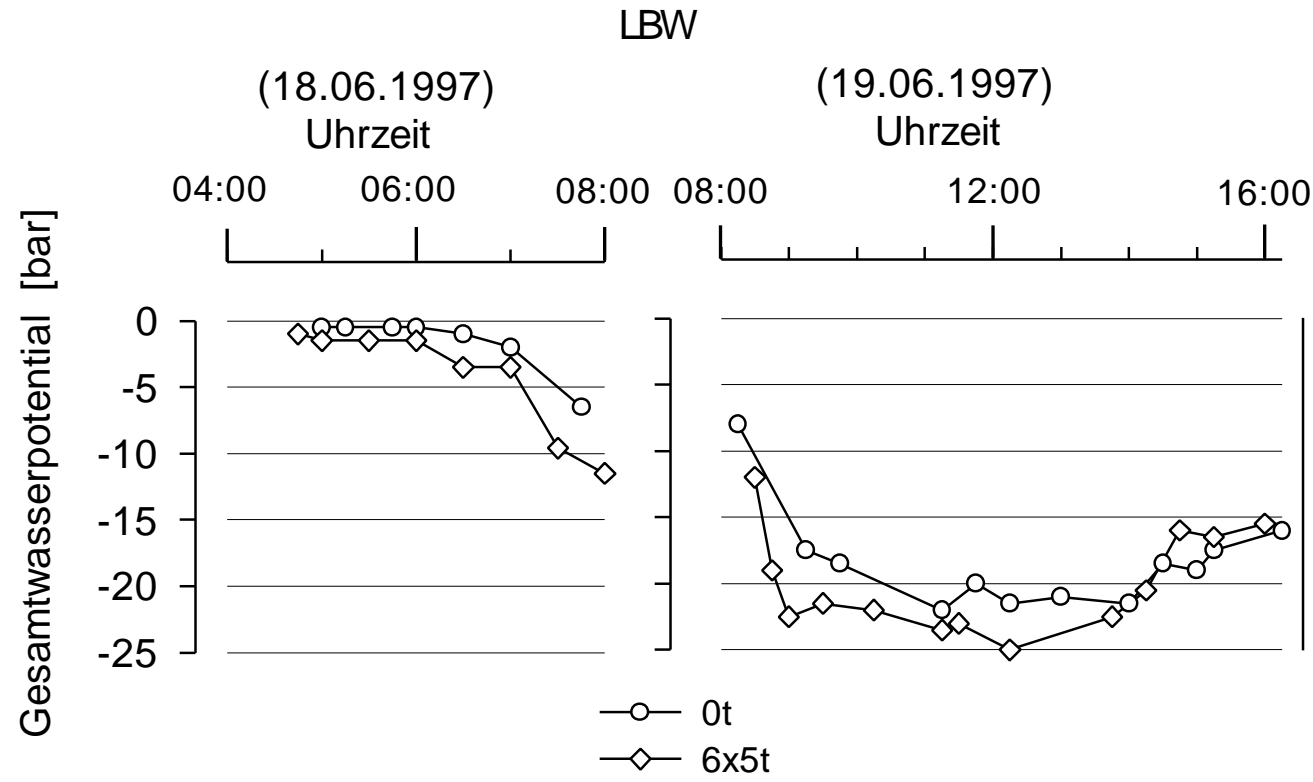

FMW

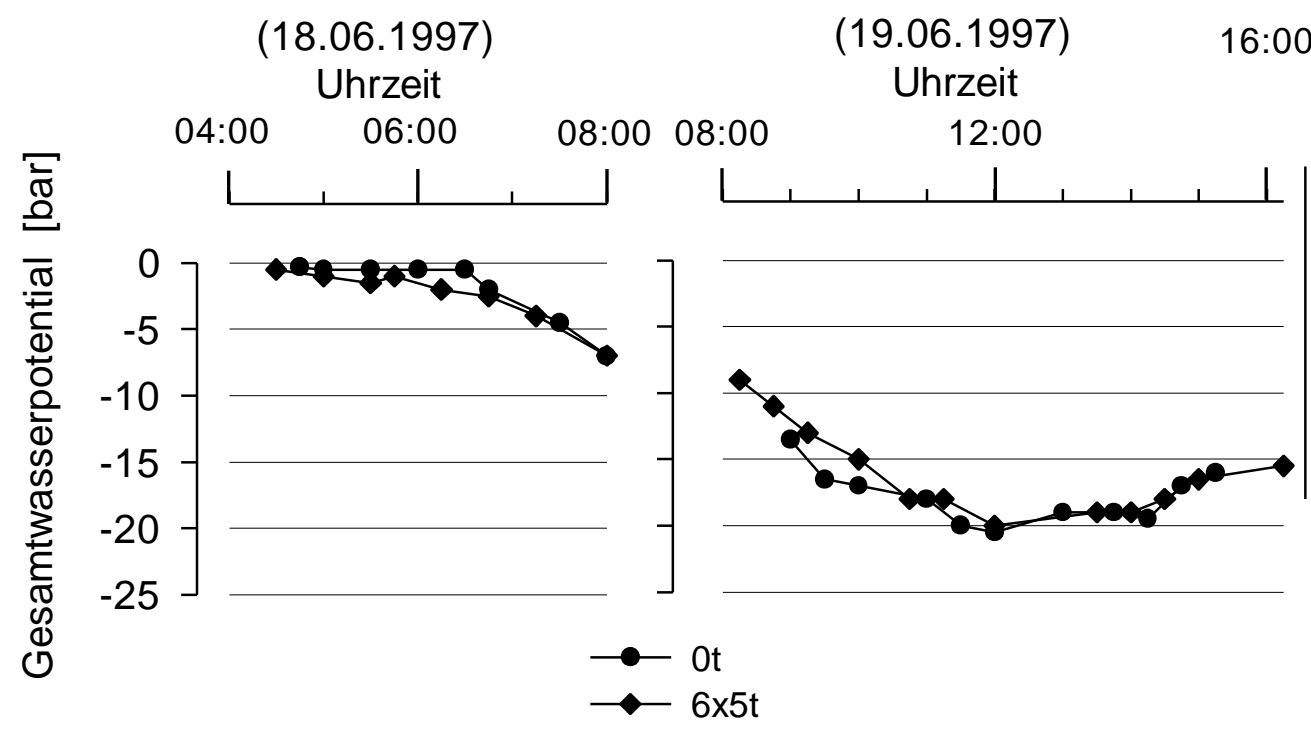

Abb. 45: Gesamtwasserpotential in der Wintergerste als Funktion der Zeit am 18. und 19.06.1996 auf dem Standort Reinshof zum Stadium EC 83. 
LBW

FMW

Bodenwasserausschöpfung [Vol.\%= $\mathrm{mm} / \mathrm{dm}$ Bodenschicht]
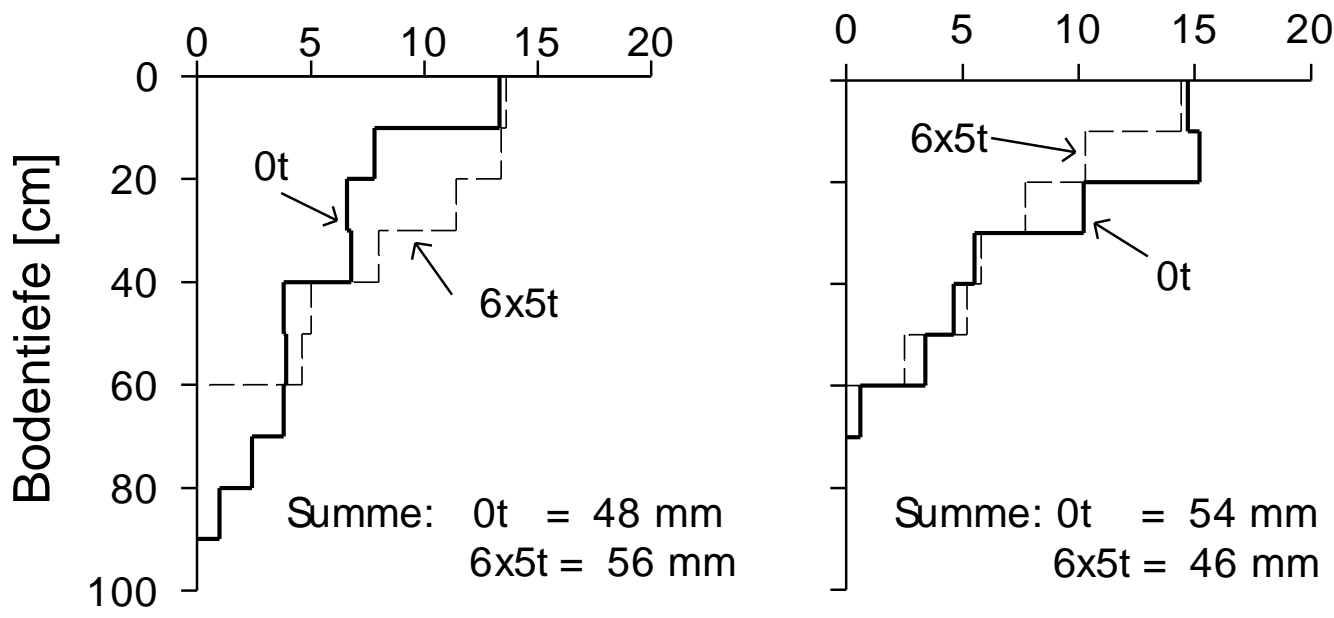

Abb. 46: Bodenwasserausschöpfung vom 04.06.1997 bis zum 19.06.1997 aus einzelnen Bodenschichten auf dem Standort Reinshof.

Die Gaswechselmessungen im Tagesgang vom 04.06.1997 bis zum 17.06.1997 (nicht dargestellt) zeigten auf dem gepflügten Boden bei hoher Bodenbelastung eine tendenziell geringere $\mathrm{CO}_{2}$-Assimilation als auf unbelastetem Boden. In diesem Zeitabschnitt lagen die Werte der $\mathrm{CO}_{2}$-Assimilation auf dem flach bearbeitet und gemulchten Boden (nicht dargestellt) bemerkenswerter Weise bei hoher Belastung über den Werten der Kontrolle (FMW 0t). Wie aus Abb. 44 (rechts) hervorgeht, übertraf die $\mathrm{CO}_{2}$-Assimilation auf dem hoch belasteten Boden am 18. und 19.06.1997 die Assimilationsraten der Kontrolle (FMW 0t). Die Differenz zwischen den Werten der Belastungsstufen 0t und 6x5t nahm jedoch im Zeitabschnitt vom 04.06.1997 bis zum 17.06.1997 ab, und am 17.06.1997 lag die $\mathrm{CO}_{2}$-Assimilation bei 0t und 6x5t annähernd auf einer Höhe. In Abb. 47 sind die Gaswechsel-Messungen vom 04.06.1997 bis zum 19.06.1997 in Lichtfluß-Effektkurven zusammengefaßt. 


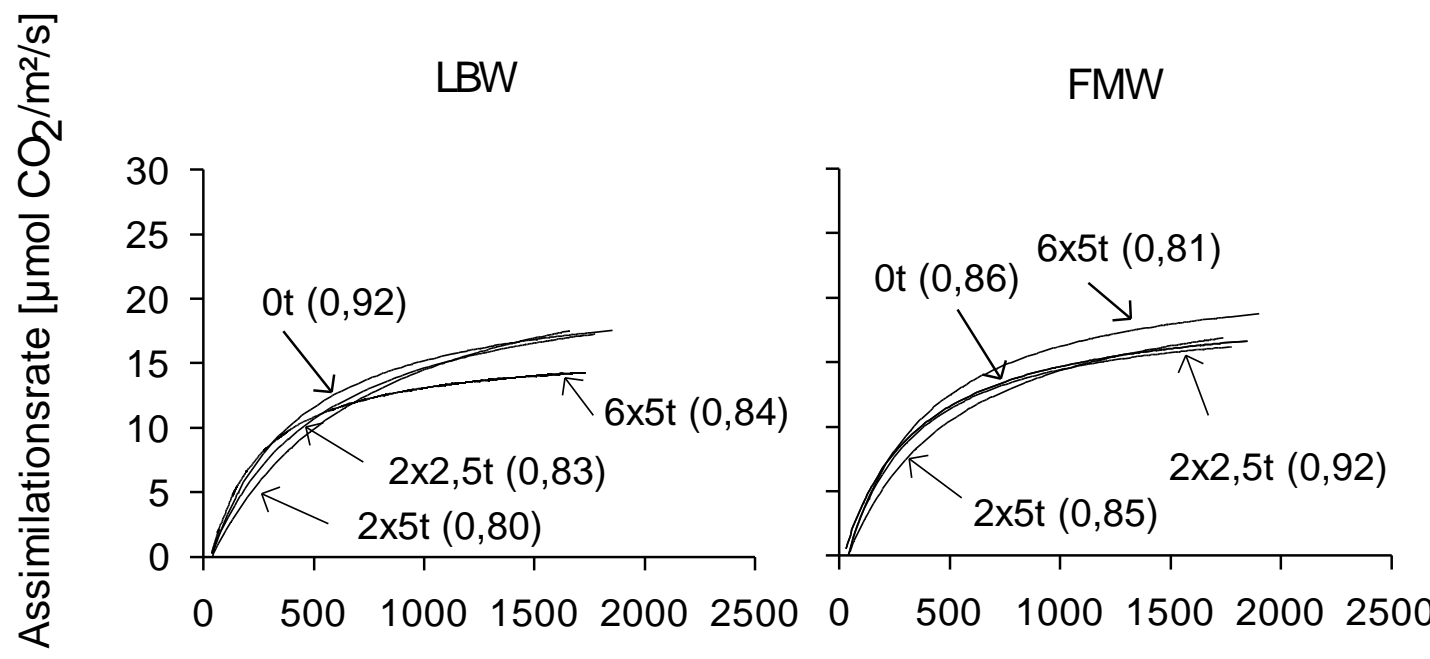

PAR $\left[\mu \mathrm{mol}\right.$ Photonen $\left./ \mathrm{m}^{2} / \mathrm{s}\right]$

Abb. 47: Lichtfluß-Effektkurven der Wintergerste zum Stadium EC 69 bis $83 \mathrm{im}$ Jahr 1997 auf dem Standort Reinshof. Die Zahlen in Klammern zeigen das Bestimmtheitsmaß $\left(\mathrm{r}^{2}\right)$ der Regression.

Auf gepflügtem Boden (Abb. 47 links) zeigte sich 1997 dieselbe Tendenz wie 1996: Bei geringer PAR $\left(<700 \mu \mathrm{mol}\right.$ Photonen $\left./ \mathrm{m}^{2} / \mathrm{s}\right)$ lag die Assimilationsrate bei allen Belastungsstufen auf gleichem Niveau. Bei einer höheren Strahlungsmenge ( $>700 \mu \mathrm{mol}$ Photonen $/ \mathrm{m}^{2} / \mathrm{s}$ ) nahmen die Pflanzen bei $6 \times 5 \mathrm{t}$ deutlich weniger $\mathrm{CO}_{2}$ pro Einheit Blattfläche und Zeit auf als die Pflanzen bei den geringeren Belastungsstufen. Bei diesen Stufen waren die Assimilationsraten über den gesamten Bereich der Strahlungsmenge annähernd identisch.

Bei FMW (Abb.47 rechts) zeigte sich eine bemerkenswerte Umkehrung der Reaktion: Im Bereich stärkerer PAR (> ca. $500 \mu \mathrm{mol}$ Photonen $/ \mathrm{m}^{2} / \mathrm{s}$ ) war die Assimilationsrate bei $6 \times 5 t$ der Rate bei den anderen Belastungsstufen überlegen. Auf dem maximal belasteten Boden nahmen die Pflanzen bei höherer Lichtmenge ca. $15 \%$ mehr $\mathrm{CO}_{2}$ pro Einheit Blattfläche und Zeit auf als die Pflanzen bei den Belastungsstufen $0 t$, 2x2,5t und $6 \times 5 t$. 


\subsection{Ertragsbildung und Ertrag}

Aus den Ertragskomponenten Körner pro Flächeneinheit und Einzelkornmasse (EKM) ergibt sich der pflanzenbauliche Flächenertrag. Die Komponente Körner pro Flächeneinheit setzt sich zusammen aus den Teilkomponenten Ährendichte (Anzahl Ähren pro Flächeneinheit) und Kornbesatz der Ähre (Anzahl Körner pro Ähre).

Die Pflanzendichte beschreibt die Anzahl der Einzelpflanzen pro Flächeneinheit vor dem Stadium des Bestockens. Sie ist das Ergebnis des Entwicklungsverlaufes des Bestandes bis zur Zählung abhängig von der Menge der ausgesäten Körner, der Keimung, dem Feldaufgang und der Entwicklung der bestockungsfähigen Pflanzen.

Im Stadium des Bestockens wird in Abhängigkeit von der Gunst der Umweltbedingungen die maximale Anzahl der Halme pro Einzelpflanze festgelegt. Nicht alle Halme können eine Blütenanlage vollständig bis zum Ende ausbilden. Viele Halme werden während des Schossens zurückgebildet. Die Ährendichte gibt an, wieviel Fruchtstände pro Flächeneinheit nach den Aufbau- und Reduktionsprozessen während der Bestockung, des Schossens und der Blüte zur Kornbildung gelangen konnten.

In ihrer Ausprägung unterliegen alle Ertragskomponenten in Abhängigkeit von Umweltfaktoren erheblichen Schwankungen. Soweit es sich bei den Umweltfaktoren um standortspezifische Klima- und Bodenbedingungen handelt, sind insbesondere das Licht, die Temperatur sowie die Wasser- und Nährstoffversorgung zu nennen.

In den vorangegangenen Kapiteln wurde dargestellt, inwieweit die Bearbeitungssysteme und die mechanische Bodenbelastung auf die bodenphysikalischen Eigenschaften, das Wurzel- und Sproßwachstum und den Gaswechsel der Kulturpflanzen Einfluß genommen haben.

Im folgenden Kapitel soll erörtert werden, in welchem Ausmaß diese Änderungen der Umweltbedingungen und die dadurch geprägte Entwicklung der Kulturpflanzen die Ertragsbildung und den Ertrag beeinflussen konnten. 


\subsubsection{Standort Reinshof}

\subsubsection{Ertragsbildung}

Im ersten Versuchsjahr wurde auf dem Standort Reinshof der Kornbesatz der Ähre nicht gezählt. In Tab. 26 werden die Ertragskomponenten und die Pflanzendichte zusammengefaßt.

Tab. 26: Ertragsbildung bei Sommergerste im Jahr 1995 auf dem Standort Reinshof. EKM bezeichnet die Einzelkornmasse. Unterschiedliche Buchstaben kennzeichnen signifikante Unterschiede zwischen den Merkmalen der Bearbeitungssysteme und der Belastungsstufen. Die Daten stammen von Block 1-4.

\begin{tabular}{|c|c|c|c|}
\hline Belastungsstufe & $\begin{array}{l}\text { Pflanzendichte } \\
\text { [Pflanzen/m²] }\end{array}$ & $\begin{array}{l}\text { Ährendichte } \\
\text { [Ähren/m²] }\end{array}$ & $\begin{array}{l}\text { EKM } \\
{[\mathrm{mg}]}\end{array}$ \\
\hline & \multicolumn{3}{|c|}{ LBW } \\
\hline Ot & $206^{a}$ & $530^{a}$ & $37,4^{a}$ \\
\hline $2 \times 2,5 t$ & $209^{a}$ & $354^{b}$ & $34,2^{a}$ \\
\hline $2 \times 5 t$ & $213^{a}$ & $265^{c}$ & $36,7^{\mathrm{a}}$ \\
\hline \multirow[t]{2}{*}{$6 \times 5 t$} & $194^{a}$ & $252^{c}$ & $33,4^{b}$ \\
\hline & \multicolumn{3}{|c|}{ FMW } \\
\hline ot & $212^{a}$ & $503^{a}$ & $37,3^{a}$ \\
\hline $2 \times 2,5 t$ & $203^{a}$ & $476^{a}$ & $36,9^{\mathrm{a}}$ \\
\hline $2 \times 5 t$ & $209^{a}$ & $435^{a}$ & $36,7^{\mathrm{a}}$ \\
\hline $6 \times 5 t$ & $200^{a}$ & $313^{b}$ & $35,5^{\mathrm{a}}$ \\
\hline
\end{tabular}

Bei LBW und FMW mit hoher Belastung zeigte sich im Vergleich zu den anderen Varianten ein verzögerter und schwächerer Feldaufgang mit lückigem Bestand (Tab. 26). Die Unterschiede in der Pflanzendichte vor dem Bestocken waren jedoch nicht signifikant. Die Anzahl der Ähren, die sich pro Flächeneinheit ausbildeten, wies auf deutliche Entwicklungsunterschiede in Abhängigkeit von der Belastung hin: Bei LBW führte schon die niedrige Bodenbelastung zu einer signifikanten Abnahme der Ährendichte. Durch die Belastung mit 2x5t und 6x5t nahm die Ährendichte noch einmal signifikant ab. Bei FMW lag die Ährendichte bei der hohen Belastungsstufe signifikant unter den Werten der anderen Belastungsstufen. 
Bis auf eine Ausnahme befanden sich die Werte der EKM auf gleichem Niveau. Nur bei hoher Bodenbelastung auf gepflügtem Boden war die EKM signifikant erniedrigt. Im zweiten Versuchsjahr wurde auf dem Standort Reinshof zusätzlich der Kornbesatz der Ähre ermittelt. Die Ertragskomponenten und die Pflanzendichte sind in Tab. 27 zusammengefaßt.

Tab. 27: Ertragsbildung bei Winterweizen im Jahr 1996 auf dem Standort Reinshof. EKM bezeichnet die Einzelkornmasse. Unterschiedliche Buchstaben kennzeichnen signifikante Unterschiede zwischen den Merkmalen der Bearbeitungssysteme und der Belastungsstufen. Die Werte stammen von Block 1-4.

\begin{tabular}{|c|c|c|c|c|c|}
\hline $\begin{array}{c}\text { Belastungs- } \\
\text { stufe }\end{array}$ & $\begin{array}{c}\text { Pflanzen- } \\
\text { dichte } \\
{\left[\text { Pflanzen } / \mathbf{m}^{2}\right]}\end{array}$ & $\begin{array}{c}\text { Ähren- } \\
\text { dichte } \\
\text { [Ähren/m²] }\end{array}$ & $\begin{array}{c}\text { Kornbesatz } \\
\text { der Ähre } \\
\text { [Körner/Ähre] }\end{array}$ & $\begin{array}{l}\text { EKM } \\
\text { [mg] }\end{array}$ & $\begin{array}{l}\text { Kornertrag } \\
\text { berechnet } \\
\text { [dt TM/ha] }\end{array}$ \\
\hline & \multicolumn{5}{|c|}{ LBW } \\
\hline $0 t$ & $414^{a}$ & $544^{a}$ & $38^{a}$ & $42,4^{a}$ & $87,6^{a}$ \\
\hline $2 \times 2,5 t$ & $408^{a}$ & $537^{a}$ & $37^{a}$ & $43,7^{a}$ & $86,8^{a}$ \\
\hline $2 \times 5 t$ & $420^{a}$ & $510^{a}$ & $37^{a}$ & $43,3^{a}$ & $81,7^{a}$ \\
\hline \multirow[t]{2}{*}{$6 \times 5 t$} & $418^{a}$ & $530^{a}$ & $33^{b}$ & $41,4^{\mathrm{a}}$ & $72,4^{b}$ \\
\hline & \multicolumn{5}{|c|}{ FMW } \\
\hline $0 t$ & $419^{a}$ & $530^{a}$ & $38^{a}$ & $41,3^{a}$ & $\overline{83,2^{a}}$ \\
\hline $2 \times 2,5 t$ & $416^{a}$ & $571^{a}$ & $35^{a}$ & $40,9^{a}$ & $81,7^{\mathrm{a}}$ \\
\hline $2 \times 5 t$ & $403^{a}$ & $516^{a}$ & $37^{a}$ & $41,9^{a}$ & $80,0^{a}$ \\
\hline $6 \times 5 t$ & $412^{a}$ & $496^{a}$ & $35^{a}$ & $41,9^{a}$ & $72,7^{b}$ \\
\hline
\end{tabular}

Die Pflanzen- und Ährendichte zeigten keine signifikanten Unterschiede zwischen den Varianten. Beim Kornbesatz der Ähren fällt der Wert bei LBW mit hoher Belastung auf, der signifikant niedriger liegt als bei allen anderen Varianten. Die hohe Bodenbelastung führte auf dem gepflügten Boden nicht nur zu einer Reduktion des Kornbesatzes der Ähre, sondern in der Tendenz auch zu einer geringeren Einzelkornmasse. Die EKM bei LBW und FMW wiesen darauf hin, daß auf dem gepflügten Boden die Kornfüllungsphase schwerere Körner hervorgebracht hat als auf dem flach bearbeitet und gemulchten Boden.

Wie schon in den beiden ersten Versuchsjahren konnte bei der Pflanzendichte im dritten Jahr kein Unterschied zwischen den Varianten nachgewiesen werden 
(Tab. 28). Die Meßwerte deuten jedoch an, daß sich bei FMW geringfügig mehr Gerstenpflanzen pro Flächeneinheit ausbilden konnten als auf dem gepflügten Boden. Innerhalb des Faktors LBW bildeten sich bei hoher Bodenbelastung signifikant weniger Ähren pro Flächeneinheit als bei den anderen drei Stufen. Auf dem flach bearbeiteten Boden fielen besonders die Varianten mit geringer und hoher Belastung auf: Bei geringer Bodenbelastung entwickelten sich erheblich weniger Ähren pro Flächeneinheit als bei der Kontrollparzelle LBW 0t. Bei hoher Bodenbelastung wurde dagegen die höchste Ährendichte ermittelt. Im Mittel über die Bearbeitungssysteme scheinen sich auf dem gepflügten Boden geringfügig mehr Ähren pro Flächeneinheit ausgebildet zu haben. Bei der Betrachtung des Kornbesatzes der Ähre stellte sich ein weiterer Effekt des Bearbeitungssystems heraus: Bei LBW lagen die Werte signifikant höher als bei FMW. Innerhalb der Bearbeitungssysteme konnte eine Wirkung der Bodenbelastung auf den Kornbesatz nicht nachgewiesen werden. Die EKM lag bei allen Varianten auf dem gleichen Niveau. Bei LBW 6x5t lag der berechnete Kornertrag unter dem Ertrag der anderen Stufen.

Tab. 28: Ertragsbildung bei Wintergerste im Jahr 1997 auf dem Standort Reinshof. EKM bezeichnet die Einzelkornmasse. Unterschiedliche Buchstaben kennzeichnen signifikante Unterschiede zwischen den Merkmalen der Bearbeitungssysteme und der Belastungsstufen. Die Werte stammen von Block 1, 3 und 4.

\begin{tabular}{|c|c|c|c|c|c|}
\hline $\begin{array}{c}\text { Belastungs } \\
\text { stufe }\end{array}$ & $\mid \begin{array}{c}\text { Pflanzen- } \\
\text { dichte } \\
\text { [Pflanzen/m²] }\end{array}$ & $\begin{array}{c}\text { Ähren- } \\
\text { dichte } \\
\left.\text { [Ähren } / \mathbf{m}^{2}\right]\end{array}$ & $\begin{array}{c}\text { Kornbesatz } \\
\text { der Ähre } \\
\text { [Körner/Ähre] }\end{array}$ & $\begin{array}{l}\text { EKM } \\
{[\mathrm{mg}]}\end{array}$ & $\begin{array}{l}\text { Kornertrag } \\
\text { berechnet } \\
\text { [dt TM/ha] }\end{array}$ \\
\hline & \multicolumn{5}{|c|}{ LBW } \\
\hline $0 t$ & $\overline{261^{a}}$ & $432^{a}$ & $36^{a}$ & $41,5^{\mathrm{a}}$ & $\overline{64,5^{a}}$ \\
\hline $2 \times 2,5 t$ & $247^{a}$ & $419^{a}$ & $38^{a}$ & $42,2^{\mathrm{a}}$ & $67,2^{\mathrm{a}}$ \\
\hline $2 \times 5 t$ & $259^{a}$ & $417^{\mathrm{a}}$ & $37^{\mathrm{a}}$ & $41,8^{\mathrm{a}}$ & $64,5^{\mathrm{a}}$ \\
\hline \multirow[t]{2}{*}{$6 \times 5 t$} & $247^{a}$ & $357^{b}$ & $39^{\mathrm{a}}$ & $41,9^{\mathrm{a}}$ & $58,3^{\mathrm{a}}$ \\
\hline & \multicolumn{5}{|c|}{ FMW } \\
\hline $0 t$ & $279^{a}$ & $364^{b}$ & $33^{b}$ & $41,3^{\mathrm{a}}$ & $49,6^{b}$ \\
\hline $2 \times 2,5 t$ & $283^{a}$ & $266^{c}$ & $27^{\mathrm{b}}$ & $40,8^{\mathrm{a}}$ & $29,3^{b}$ \\
\hline $2 \times 5 t$ & $265^{a}$ & $340^{b}$ & $28^{\mathrm{b}}$ & $40,6^{a}$ & $38,6^{\mathrm{b}}$ \\
\hline $6 \times 5 t$ & $264^{\mathrm{a}}$ & $496^{a}$ & $31^{\mathrm{b}}$ & $41,1^{\mathrm{a}}$ & $63,2^{\mathrm{a}}$ \\
\hline
\end{tabular}




\subsubsection{Ertrag}

Auf beiden Bearbeitungssystemen konnte im ersten Versuchsjahr 1995 eine Abhängigkeit des Ertrags von der Belastungsstufe gefunden werden (Abb. 48 oben). Bei LBW lag der Kornertrag der Sommergerste der Kontrollparzelle (LBW 0t) signifikant über den Erträgen bei geringer, mittlerer und hoher Bodenbelastung. In der Tendenz zeigte sich eine deutliche Abnahme des Kornertrages mit steigender Bodenbelastung. Bei FMW dagegen stieg der Kornertrag von der ersten bis zur dritten Belastungsstufe an (Abb. 48 oben rechts). Diese Unterschiede waren nicht signifikant, wohl aber die Reduktion des Kornertrages durch die hohe Bodenbelastung.

Wie Tab. 29 für die Sommergerste zeigt, änderte sich der Ernteindex - als Maß für die Assimilatverteilung in der Pflanze - bei steigender mechanischer Bodenbelastung erheblich. Bei LBW wurden in der Kornfüllungsphase mit zunehmender Bodenbelastung weniger Assimilate in das Korn im Vergleich zu anderen Organen eingelagert. Bei FMW fällt besonders die Variante mit mittlerer Bodenbelastung auf: Hier zeigte sich nicht nur der tendenziell höchste Kornertrag, sondern auch der höchste Ernteindex. Im Vergleich zu den anderen Varianten wurden erheblich mehr Assimilate in das Korn eingelagert als in das Stroh. 
LBW

FMW

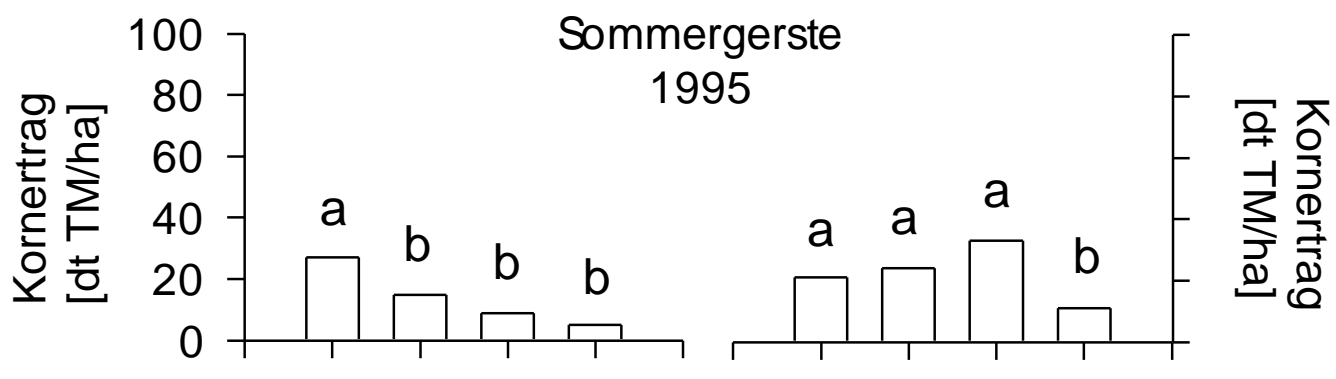

Winterweizen

1996
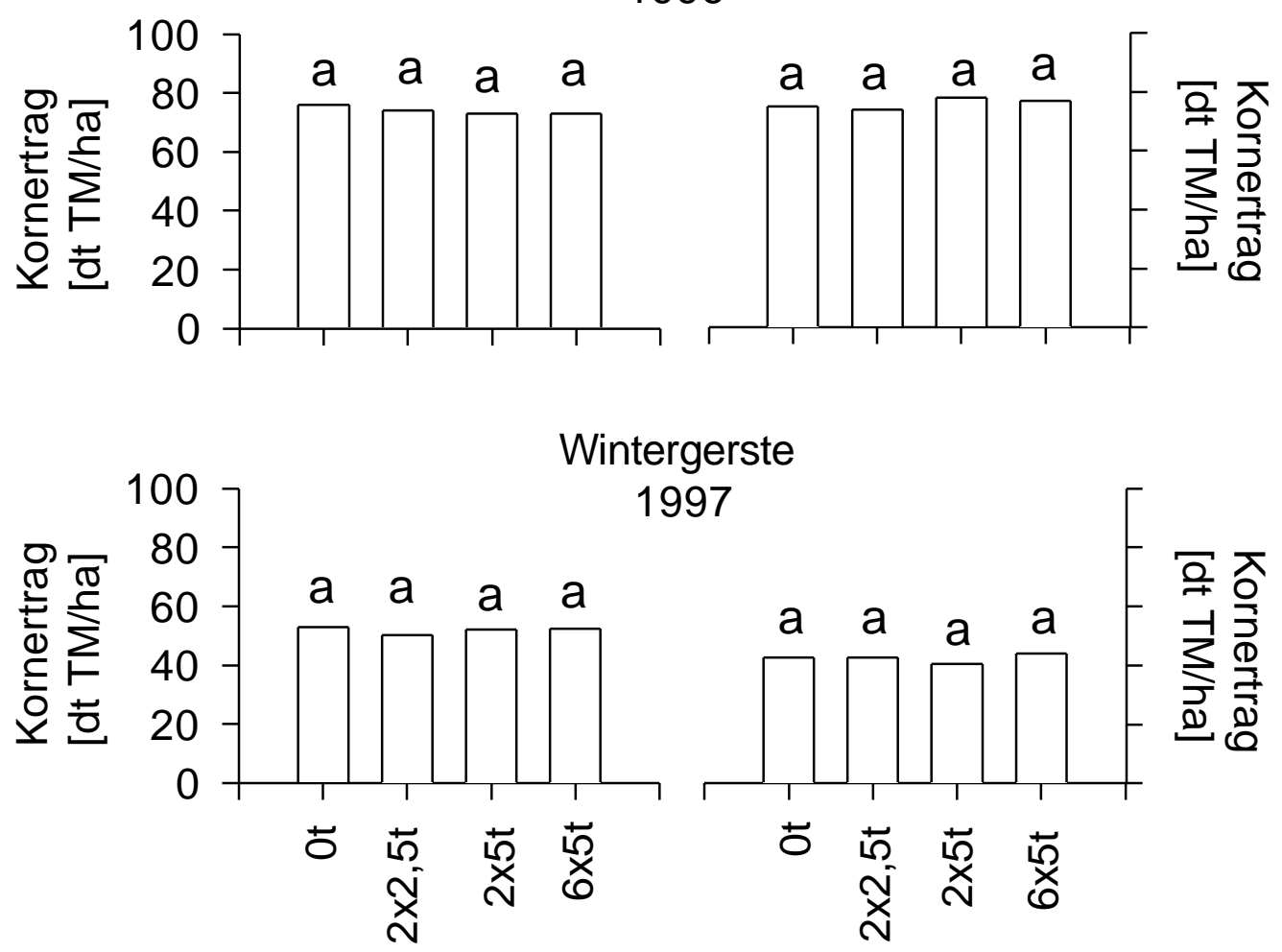

Abb. 48: Kornertrag in den drei Versuchsjahren auf dem Standort Reinshof. Unterschiedliche Buchstaben kennzeichnen signifikante Unterschiede zwischen den Merkmalen der Bearbeitungssysteme und der Belastungsstufen. Die Werte stammen für die Jahre 1995 und 1996 von Block 1-4 und für 1997 von Block 1, 3 und 4. 
Tab. 29: Ernteindex bei Sommergerste (SG) 1995 , Winterweizen (WW) 1996 und Wintergerste (WG) 1997 auf dem Standort Reinshof.

\begin{tabular}{cc|cccc} 
& & \multicolumn{4}{|c}{ Ernteindex } \\
\hline $\begin{array}{c}\text { Kulturpflanze/ } \\
\text { Jahr }\end{array}$ & $\begin{array}{c}\text { Bearbeitungs- } \\
\text { system }\end{array}$ & \multicolumn{4}{|c}{ Belastungsstufe } \\
SG/1995 & LBW & 0,41 & $2 \times 2,5 \mathrm{t}$ & $2 \times 5 \mathrm{t}$ & $6 \times 5 \mathrm{t}$ \\
& FMW & 0,36 & 0,33 & 0,32 & 0,28 \\
WW/1996 & LBW & 0,58 & 0,57 & 0,45 & 0,31 \\
& FMW & 0,57 & 0,56 & 0,58 & 0,58 \\
WG/1997 & LBW & 0,53 & 0,54 & 0,56 & 0,57 \\
& FMW & 0,48 & 0,46 & 0,46 & 0,52 \\
& & & & &
\end{tabular}

Im zweiten Versuchsjahr konnte bei Winterweizen keine Beeinflussung des gemessenen Kornertrags durch das Bearbeitungssystem oder durch die Belastungsstufen nachgewiesen werden (Abb. 48 mitte). Wie aus Tab. 29 hervorgeht, verliefen in der Kornfüllungsphase die Translokationsprozesse bei allen Varianten nach ähnlichem Muster.

So wie im zweiten Versuchsjahr, so zeigte sich auch 1997 beim Kornertrag der Wintergerste keine signifikante Differenz zwischen den Varianten. Tendenziell lag der Kornertrag bei FMW um ca. 20\% unter dem Kornertrag bei LBW. Der Ernteindex für die Wintergerste in Tab. 29 deutet an, daß bei FMW weniger Assimilate in das Korn im Vergleich zu anderen Organen eingelagert wurden als bei LBW.

\subsubsection{Standort Relliehausen}

\subsubsection{Ertragsbildung}

Wie Tab. 30 zeigt, deutet die Anzahl der Pflanzen (Sommergerste) pro Flächeneinheit darauf hin, daß die hohe Bodenbelastung auf dem gepflügten Boden - verglichen mit den anderen Belastungsstufen - zu einer etwas geringeren Pflanzendichte führte. Auf dem flach bearbeiteten Boden zeigte die Pflanzendichte keine grundlegende Abhängigkeit von der Höhe der Bodenbelastung. 
Tab. 30: Ertragsbildung bei Sommergerste im Jahr 1995 auf dem Standort Relliehausen. EKM bezeichnet die Einzelkornmasse. Die angegebenen Werte sind Mittelwerte der Meßwiederholungen auf den Parzellen.

\begin{tabular}{|c|c|c|c|}
\hline Belastungsstufe & $\begin{array}{l}\text { Pflanzendichte } \\
\text { [Pflanzen/m²] }\end{array}$ & $\begin{array}{l}\text { Ährendichte } \\
\text { [Ähren/m²] }\end{array}$ & $\begin{array}{l}\text { EKM } \\
{[\mathrm{mg}]}\end{array}$ \\
\hline & \multicolumn{3}{|c|}{ LBW } \\
\hline $0 t$ & 202 & 432 & 44,2 \\
\hline $2 \times 2,5 t$ & 204 & 440 & 45,5 \\
\hline $2 \times 5 t$ & 205 & 445 & 44,2 \\
\hline \multirow[t]{2}{*}{$6 \times 5 t$} & 190 & 400 & 42,5 \\
\hline & \multicolumn{3}{|c|}{ FMW } \\
\hline $0 t$ & 204 & 422 & 43,3 \\
\hline $2 \times 2,5 t$ & 183 & 480 & 42,4 \\
\hline $2 \times 5 t$ & 204 & 433 & 43,0 \\
\hline $6 \times 5 t$ & 197 & 414 & 44,0 \\
\hline
\end{tabular}

Im weiteren Verlauf der Vegetation bildeten sich sowohl bei LBW als auch bei FMW auf dem hoch belasteten Boden weniger Ähren pro Flächeneinheit aus als bei den anderen Belastungsstufe. Die Meßwerte für EKM deuten an, daß die Kornfüllungsphase auf dem gepflügten Boden durch die hohe mechanische Bodenbelastung negativ beeinflußt wurde. Bei FMW scheint die Bodenbelastung keinen Einfluß auf die EKM gehabt zu haben. Auffällig ist die Variante FMW 2x2,5t. Trotz der geringeren Bodenbelastung lag die EKM unter dem Wert bei FMW 6x5t.

Im Versuchsjahr 1996 wurde bei LBW eine Zunahme der Pflanzendichte mit steigender Bodenbelastung beobachtet (Tab. 31). 
Tab. 31: Ertragsbildung bei Winterweizen im Jahr 1996 auf dem Standort Relliehausen. EKM bezeichnet die Einzelkornmasse. Die angegebenen Werte sind Mittelwerte der Meßwiederholungen auf den Parzellen.

\begin{tabular}{|c|c|c|c|}
\hline Belastungsstufe & $\begin{array}{l}\text { Pflanzendichte } \\
\text { [Pflanzen/m²] }\end{array}$ & $\begin{array}{l}\text { Ährendichte } \\
\text { [Ähren/m²] }\end{array}$ & $\begin{array}{l}\text { EKM } \\
\text { [mg] }\end{array}$ \\
\hline & \multicolumn{3}{|c|}{ LBW } \\
\hline $\mathbf{0 t}$ & 204 & 370 & 37,2 \\
\hline $2 \times 2,5 t$ & 221 & 411 & 36,0 \\
\hline $2 \times 5 t$ & 238 & 292 & 32,2 \\
\hline \multirow[t]{2}{*}{$6 \times 5 t$} & 238 & 382 & 31,0 \\
\hline & \multicolumn{3}{|c|}{ FMW } \\
\hline $\mathbf{0 t}$ & 280 & 409 & 41,2 \\
\hline $2 \times 2,5 t$ & 263 & 515 & 41,6 \\
\hline $2 \times 5 t$ & 255 & 408 & 37,2 \\
\hline $6 \times 5 t$ & 255 & 447 & 40,7 \\
\hline
\end{tabular}

Die Pflanzendichte auf gepflügtem Boden lag bei hoher Belastung gegenüber der Kontrolle (LBW 0t) um 17\% höher (Tab. 31). Auf dem flach bearbeiteten Boden stellte sich die Pflanzendichte anders dar: Mit steigender Bodenbelastung nahm die Anzahl Pflanzen pro Flächeneinheit ab. Die hohe Bodenbelastung führte zu einer Reduktion der Pflanzendichte um ca. 10\%. Insgesamt lag die Pflanzendichte höher als bei LBW (Tab. 31).

Auf dem gepflügten Boden hatte die Belastung kaum einen Einfluß auf die Ährendichte, wohl aber auf die EKM. Mit steigender Bodenbelastung nahm die EKM erheblich ab. Bei FMW deutete sich eine reduzierende Auswirkung der Belastung auf die Ährendichte und die EKM nicht an (Tab. 31).

Bei einem Vergleich der Bearbeitungssysteme - gemittelt über die Belastungsstufen - zeigte sich eine bemerkenswerte Entwicklung: Die Ausprägung des Bestandes schien von der Pflanzendichte bis hin zur EKM auf dem flach bearbeiteten Boden wesentlich günstiger verlaufen zu sein als auf dem gepflügten Boden.

Im dritten Versuchsjahr wurde auf Grund des hohen Arbeits- und Zeitaufwandes auf die Erfassung der Ertragsbildung an diesem Standort verzichtet. 


\subsubsection{Ertrag}

Im ersten Versuchsjahr konnte bei LBW eine tendenzielle Abnahme des Kornertrages der Sommergerste bei steigender Bodenbelastung festgestellt werden (Abb. 49 oben links). Die Differenz zwischen LBW 0t und LBW 6x5t betrug rund $55 \%$. Bei FMW (Abb. 49 oben rechts) lag der Kornertrag der Stufen 0t und 2x2,5t auf gleichem Niveau. Durch die mechanische Bodenbelastung wurde der Kornertrag bei mittlerer Belastung um ca. $40 \%$ und bei hoher Belastung um ca. $12 \%$ reduziert.

So wie der Kornertrag auf dem gepflügten Boden, so wies auch der Ernteindex (Tab. 32) bei LBW mit steigender Bodenbelastung fallende Werte auf. Bei FMW spiegelte sich der auffallend geringe Kornertrag der Stufe 2x5t im Ernteindex wider: Im Vergleich zu den anderen Belastungsstufen wurden weniger Assimilate im Korn als in andere Organe eingelagert. Im zweiten Versuchsjahr zeigte der Kornertrag des Winterweizens bei LBW von 0t nach 2x2,5t einen geringen Anstieg. Bei mittlerer und hoher Belastung fielen die Kornerträge unter das Niveau der unbelasteten Parzelle. Bei FMW lagen die Erträge der Belastungsstufen 0t, 2x2,5t und 2x5t auf einer Höhe. Dagegen war auf dem hoch belasteten Boden der Kornertrag verglichen mit der Kontrolle (0t) um 13\% geringer. Gemittelt über die Belastungsstufen erwies sich der flach bearbeitet und gemulchte Boden als erheblich ertragreicher: Der mittlere Kornertrag bei FMW lag um $56 \%$ höher als bei LBW (Abb.49 mitte).

Der Ernteindex 1996 deutete an, daß bei beiden Bearbeitungssystemen mit steigender Bodenbelastung weniger Assimilate in das Korn als in andere Pflanzenteile eingelagert wurden (Tab. 32). 
LBW

FMW
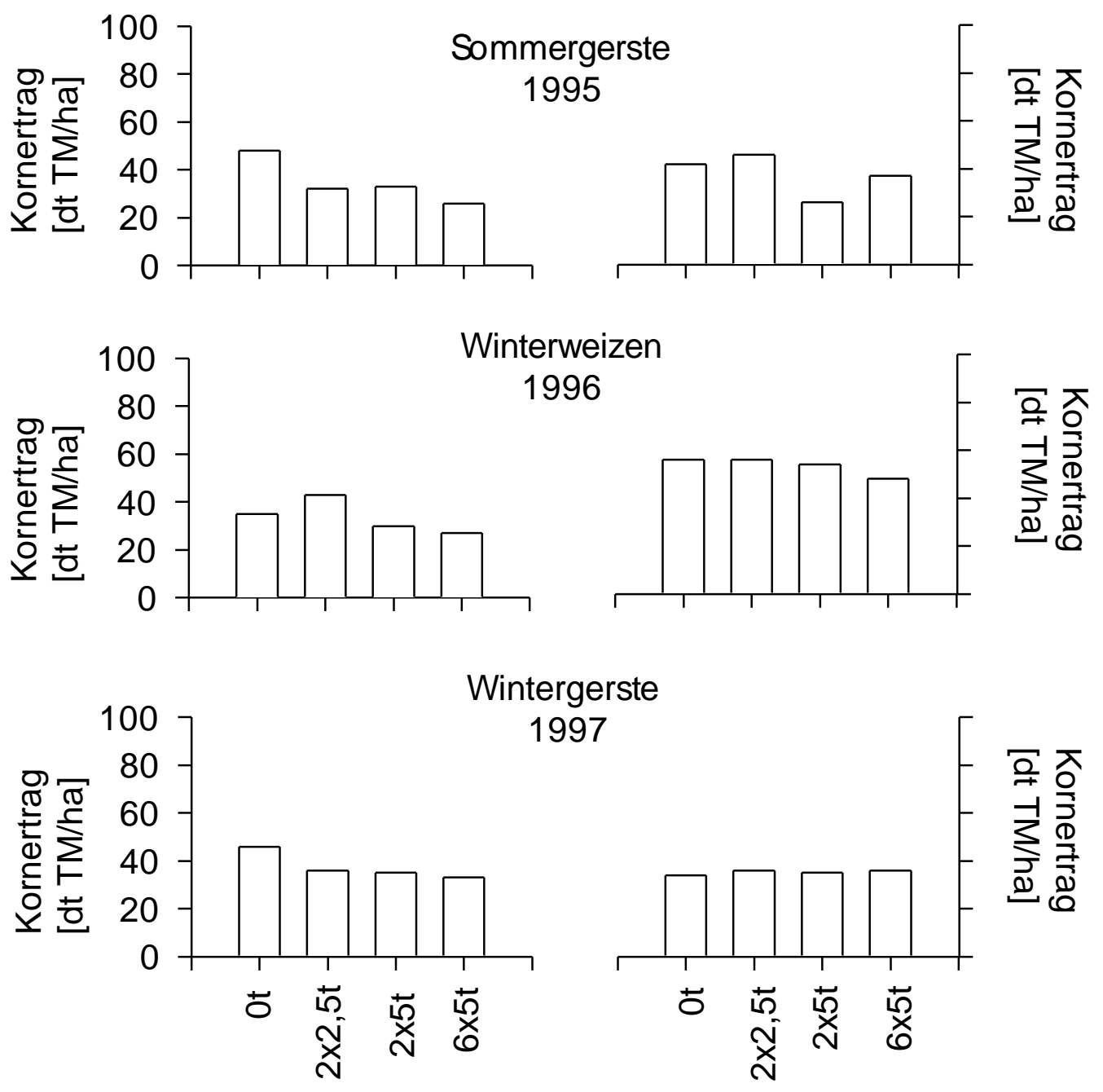

Abb. 49: Kornertrag in den drei Versuchsjahren auf dem Standort Relliehausen. Dargestellt sind die Meßergebnisse von Mischproben.

Im dritten Versuchsjahr lag der Ertrag der Wintergerste bei LBW 0t um ca. 25\% über dem Kornertrag der anderen sieben Varianten (Abb. 49 unten links). Wie Tab.32 zeigt, lagen die Ernteindizes bei FMW bemerkenswert unter denen von LBW. Bei gleichem Kornertrag bildeten die Pflanzen bei FMW beträchtlich mehr Stroh. 
Tab. 32: Ernteindex bei Sommergerste (SG) 1995, Winterweizen (WW) 1996 und Wintergerste (WG) 1997 auf dem Standort Relliehausen.

\begin{tabular}{cc|cccc} 
& & \multicolumn{4}{|c}{ Ernteindex } \\
\hline $\begin{array}{c}\text { Kulturpflanze/ } \\
\text { Jahr }\end{array}$ & $\begin{array}{c}\text { Bearbeitungs } \\
\text { system }\end{array}$ & \multicolumn{4}{|c}{ Belastungsstufe } \\
SG/1995 & LBW & 0,57 & 0,54 & 0,52 & 0,52 \\
& FMW & 0,56 & 0,55 & 0,47 & 0,54 \\
WW/1996 & LBW & 0,41 & 0,41 & 0,36 & 0,36 \\
& FMW & 0,44 & 0,44 & 0,41 & 0,42 \\
WG/1997 & LBW & 0,46 & 0,41 & 0,40 & 0,47 \\
& FMW & 0,33 & 0,35 & 0,37 & 0,35
\end{tabular}

\subsubsection{Standort Großobringen}

\subsubsection{Ertragsbildung}

Auf dem Standort Großobringen lag im ersten Versuchsjahr die Pflanzendichte der Sommmergerste vor dem Bestocken bei LBW tief mit geringer und mittlerer Belastung, sowie bei LBW flach mit den ersten drei Belastungsstufen auf gleichem Niveau (Tab. 33). Die Pflanzendichte bei LBW tief mit keiner und hoher Bodenbelastung lag deutlich unter dem Wert der anderen Varianten. LBW flach 6x5t nahm eine mittlere Stellung ein. Bei der Ährendichte war auf dem tief gepflügten Boden mit steigender Bodenbelastung eine merkliche Reduktion zu erkennen. So wie bei LBW tief, so wurde auch bei LBW flach die Ährendichte mit steigender Belastung reduziert. Bei flach bearbeitetem Boden stellte sich die Reduktion des Kennwertes bei geringer Bodenbelastung stärker dar als bei hoher Belastung.

Bei LBW tief mit keiner und geringer Belastung führte die Entwicklung der Getreidepflanzen zu einer geringfügig höheren EKM als bei mittlerer und hoher Belastung. Auf dem flach bearbeiteten Boden lag die EKM bei hoher Bodenbelastung unter der EKM der anderen Varianten dieses Bearbeitungssystems. 
Tab. 33: Ertragsbildung der Sommergerste im Jahr 1995 auf dem Standort Großobringen. EKM bezeichnet die Einzelkornmasse. Die angegebenen Werte sind Mittelwerte der Meßwiederholungen auf den Parzellen.

\begin{tabular}{|c|c|c|c|}
\hline Belastungsstufe & $\begin{array}{l}\text { Pflanzendichte } \\
\text { [Pflanzen/m²] }\end{array}$ & $\begin{array}{l}\text { Ährendichte } \\
\text { [Ähren/m²] }\end{array}$ & $\begin{array}{l}\text { EKM } \\
\text { [mg] }\end{array}$ \\
\hline & \multicolumn{3}{|c|}{ LBW tief } \\
\hline Ot & 397 & 473 & 38,5 \\
\hline $2 \times 2,5 t$ & 465 & 455 & 39,0 \\
\hline $2 \times 5 t$ & 453 & 449 & 37,8 \\
\hline \multirow[t]{2}{*}{$6 \times 5 t$} & 398 & 433 & 37,4 \\
\hline & \multicolumn{3}{|c|}{ LBW flach } \\
\hline $\mathbf{0 t}$ & 452 & 481 & 36,4 \\
\hline $2 \times 2,5 t$ & 434 & 428 & 39,3 \\
\hline $2 \times 5 t$ & 432 & 451 & 38,6 \\
\hline $6 \times 5 t$ & 412 & 441 & 32,8 \\
\hline
\end{tabular}

Die Ertragsbildung des zweiten Versuchsjahres faßt Tab. 34 zusammen. Auf dem tief gepflügten Boden nahm die Pflanzendichte von 0t nach 2x2,5t um $20 \%$ zu. Bei mittlerer und hoher Bodenbelastung bildeten sich $9 \%$ mehr Pflanzen pro Flächeneinheit als bei der Kontrolle (LBW tief 0 t). Auf dem flach gepflügten Boden fiel die Pflanzendichte im Vergleich zur Kontrolle (LBW flach 0t) bei geringer und mittlere Bodenbelastung um rund $17 \%$ ab. Bei hoher Belastung lag die Pflanzendichte 17,5\% über der Pflanzendichte auf dem Boden ohne Belastung.

Bei beiden Bearbeitungssystemen deuteten die Meßwerte darauf hin, daß sich bei hoher Bodenbelastung eine höhere Ährendichte ausbilden konnte als bei den geringeren Belastungsstufen. Auf dem flach bearbeiteten Boden mit mittlerer Belastung schienen die Bedingungen für eine hohe Ährendichte günstiger gewesen zu sein als bei einer hohen Belastung. Bei LBW tief führte das Wachstum der Pflanze bei hoher Bodenbelastung nicht nur zu einer höheren Ährendichte, sondern auch zu einem höheren Kornbesatz der Ähren. Bei flacher Bodenbearbeitung lag die Anzahl Körner pro Ähre bei geringer und hoher Bodenbelastung deutlich unter den Werte der Kontrollparzelle (LBW flach 0t). 
Bemerkenswert ist die Entwicklung bei mittlerer Belastung: Bei dieser Variante entwickelten sich pro Ähre ebensoviel Körner wie auf dem Boden mit keiner Belastung.

Die EKM bei tiefer Bodenbearbeitung deutete eine geringfügige Abnahme des Korngewichts mit steigender Bodenbelastung an. Bei LBW flach lagen die Werte aller Belastungsstufen auf einem Niveau. Allein die mittlere Belastungsstufe wies eine merklich höhere EKM auf.

Tab. 34: Ertragsbildung bei Winterweizen im Jahr 1996 auf dem Standort Großobringen. EKM bezeichnet die Einzelkornmasse. Die angegebenen Werte sind Mittelwerte der Meßwiederholungen auf den Parzellen.

\begin{tabular}{|c|c|c|c|c|c|}
\hline $\begin{array}{c}\text { Belastungs- } \\
\text { stufe }\end{array}$ & $\begin{array}{c}\text { Pflanzen- } \\
\text { dichte } \\
\text { [Pflanzen/m²] }\end{array}$ & $\begin{array}{c}\text { Ähren- } \\
\text { dichte } \\
\left.\text { [Ähren } / \mathrm{m}^{2}\right]\end{array}$ & $\begin{array}{c}\text { Kornbesatz } \\
\text { der Ähre } \\
\text { [Körner/Ähre] }\end{array}$ & $\begin{array}{l}\text { EKM } \\
\text { [mg] }\end{array}$ & $\begin{array}{l}\text { Kornertrag } \\
\text { berechnet } \\
\text { [dt TM/ha] }\end{array}$ \\
\hline & \multicolumn{5}{|c|}{ LBW tief } \\
\hline $0 t$ & 280 & 472 & 36 & 39,2 & 66,6 \\
\hline $2 \times 2,5 t$ & 336 & 447 & 37 & 35,2 & 58,2 \\
\hline $2 \times 5 t$ & 304 & 472 & 36 & 38,5 & 65,4 \\
\hline \multirow[t]{2}{*}{$6 \times 5 t$} & 304 & 516 & 40 & 38,2 & 78,8 \\
\hline & \multicolumn{5}{|c|}{ LBW flach } \\
\hline $0 t$ & 320 & 343 & 44 & 37,4 & 56,4 \\
\hline $2 \times 2,5 t$ & 264 & 374 & 39 & 37,5 & 54,7 \\
\hline $2 \times 5 t$ & 272 & 427 & 44 & 39,1 & 73,5 \\
\hline $6 \times 5 t$ & 376 & 407 & 38 & 37,0 & 57,2 \\
\hline
\end{tabular}

Die Pflanzendichte der Wintergerste im dritten Versuchsjahr (Tab. 35) lag auf dem tief bearbeiteten Boden mit geringer und mittlerer Belastung erheblich höher als bei keiner und hoher Belastung. Bei flacher Bodenbearbeitung war eine merkliche Abnahme der Pflanzendichte mit steigender Bodenbelastung festzustellen. Sowohl bei LBW tief als auch bei LBW flach deutete sich mit steigender Belastung eine Reduktion der Ährendichte an. Auf LBW tief bei der Anzahl Körner pro Ähre kehrte sich diese Tendenz um: Die hohe Belastungsstufe wies einen deutlich höheren Wert auf als die anderen Varianten. Auf dem flach be- 
arbeiteten Boden bildeten sich bei keiner und geringer Belastung beträchtlich mehr Körner pro Ähre als bei mittlerer und hoher Bodenbelastung.

Verglichen mit 2x5t und 6x5t konnte bei LBW tief 0t und 2x2,5t eine geringfügig höhere EKM festgestellt werden. Bei LBW flach lag das Korngewicht bei 0t und 6x5t auf einem tieferen Niveau als bei den Stufen 2x2,5t und 2x5t.

Tab. 35: Ertragsbildung bei Wintergerste im Jahr 1997 auf dem Standort Großobringen. EKM bezeichnet die Einzelkornmasse. Die angegebenen Werte sind Mittelwerte der Meßwiederholungen auf den Parzellen.

\begin{tabular}{|c|c|c|c|c|c|}
\hline $\begin{array}{c}\text { Belastungs- } \\
\text { stufe }\end{array}$ & $\mid \begin{array}{c}\text { Pflanzen- } \\
\text { dichte } \\
{[\text { Pflanzen/m²] }}\end{array}$ & $\begin{array}{c}\text { Ähren- } \\
\text { dichte } \\
\text { [Ähren/m²] }\end{array}$ & $\begin{array}{c}\text { Kornbesatz } \\
\text { der Ähre } \\
\text { [Körner/Ähre] }\end{array}$ & $\begin{array}{l}\text { EKM } \\
\text { [mg] }\end{array}$ & $\begin{array}{l}\text { Kornertrag } \\
\text { berechnet } \\
\text { [dt TM/ha] }\end{array}$ \\
\hline & \multicolumn{5}{|c|}{ LBW tief } \\
\hline $0 t$ & 397 & 473 & 34 & 38,5 & 61,9 \\
\hline $2 \times 2,5 t$ & 465 & 455 & 31 & 39,0 & 55,0 \\
\hline $2 \times 5 t$ & 453 & 449 & 32 & 37,8 & 54,3 \\
\hline \multirow[t]{2}{*}{$6 \times 5 t$} & 398 & 433 & 37 & 37,4 & 59,9 \\
\hline & \multicolumn{5}{|c|}{ LBW flach } \\
\hline $0 t$ & 452 & 481 & 39 & 36,4 & $\overline{68,3}$ \\
\hline $2 \times 2,5 t$ & 434 & 428 & 38 & 39,3 & 64,0 \\
\hline $2 \times 5 t$ & 432 & 451 & 29 & 38,6 & 50,5 \\
\hline $6 \times 5 t$ & 412 & 441 & 32 & 32,8 & 46,3 \\
\hline
\end{tabular}




\subsubsection{Ertrag}

Im Jahr 1995 zeigte sich bei beiden Bearbeitungssystemen eine kennzeichnende Tendenz: mit steigender Bodenbelastung fiel der Kornertrag der Sommergerste stark ab (Abb. 50 mitte). Das Ausmaß der Reduktion und das Niveau des Ertrages bei den einzelnen Belastungsstufen stellte sich bei tiefer und flacher Bodenbearbeitung gleich dar. Wie der Ernteindex 1995 in Tab. 36 andeutet, verlief die Einlagerung von Assimilaten in das Korn bei allen Varianten ähnlich. Auffällig ist die Variante LBW flach $6 \times 5$ t. Hier wurde ein deutlich geringerer Index ermittelt.

Im zweiten Versuchsjahr mit Winterweizen deutete sich bei LBW tief und LBW flach eine geringe Zunahme des Kornertrages mit steigender Bodenbelastung an (Abb. 50 oben). Bei einem Vergleich der Faktoren stellte sich bei LBW tief der Kornertrag als geringfügig höher dar als der Ertrag bei LBW flach. Innerhalb der Faktoren konnte bei tiefer Bodenbearbeitung eine Abnahme des Ernteindexes mit steigender Bodenbelastung festgestellt werden (Tab. 36). Bei flacher Bearbeitung lag der Ernteindex auf einem Niveau. So wie die Erträge , so liegen auch die Ernteindizes bei LBW tief etwas höher als bei LBW flach.

Mit steigender Bodenbelastung nahm im dritten Versuchsjahr bei beiden Systemen der Kornertrag der Wintergerste von 0t nach 6x5t um ca. $15 \%$ ab (Abb. 50 unten). Wie Tab. 36 zeigt, lagen die Ernteindizes aller Varianten auf einem Niveau. 
LBW tief

LBW flach

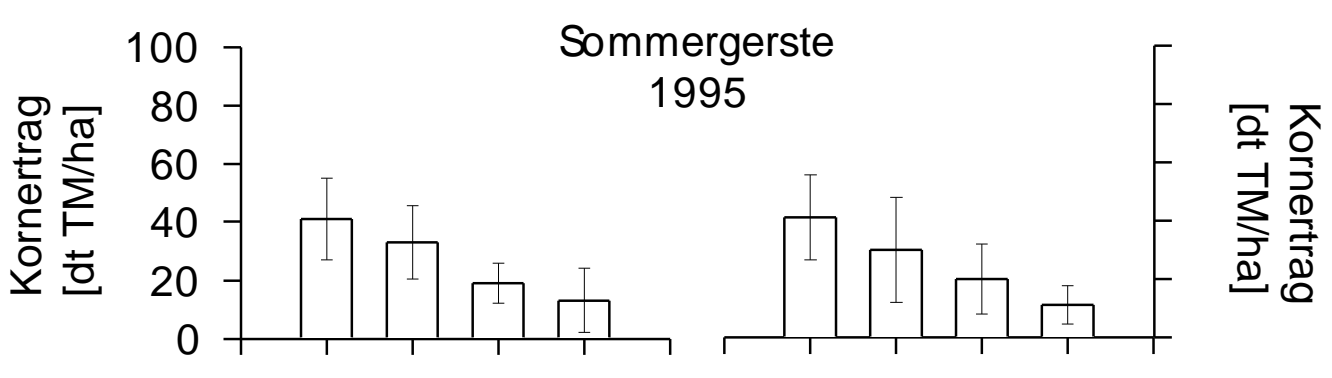

Winterweizen
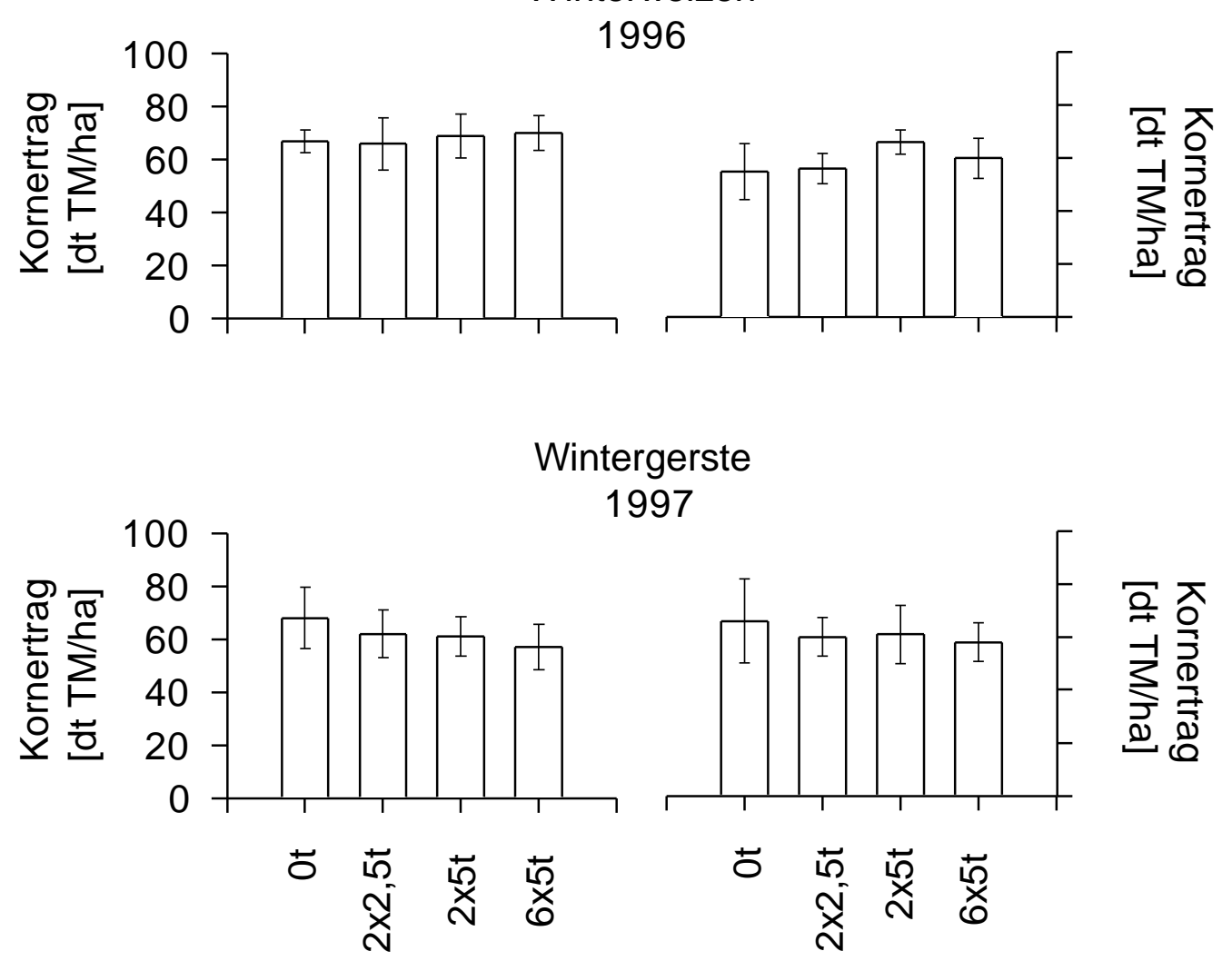

Abb. 50: Kornertrag der drei Versuchsjahre auf dem Standort Großobringen.

Senkrechte Striche kennzeichnen den 95\% Vertrauensbereich. 
Tab. 36: Ernteindex bei Sommergerste (SG) 1995, Winterweizen (WW) 1996 und Wintergerste (WG) 1997 auf dem Standort Großobringen.

\begin{tabular}{c|ccccc}
$\begin{array}{c}\text { Kulturpflanze/ } \\
\text { Jahr }\end{array}$ & $\begin{array}{c}\text { Bearbei- } \\
\text { tungs- } \\
\text { system }\end{array}$ & \multicolumn{5}{|c}{ Ernteindex } \\
& & \multicolumn{4}{c}{ Belastungsstufe } \\
& & $0 t$ & $2 \times 2,5 t$ & $2 \times 5 t$ & $6 \times 5 t$ \\
\cline { 2 - 6 } SG/1995 & LBW & 0,59 & 0,56 & 0,56 & 0,59 \\
& FMW & 0,59 & 0,56 & 0,59 & 0,52 \\
WW/1996 & LBW & 0,48 & 0,46 & 0,45 & 0,45 \\
& FMW & 0,42 & 0,43 & 0,44 & 0,43 \\
WG/1997 & LBW & 0,57 & 0,57 & 0,59 & 0,59 \\
& FMW & 0,57 & 0,59 & 0,60 & 0,57
\end{tabular}




\section{Diskussion}

Die in den letzten Jahrzehnten stark angestiegene mechanische Belastung der Ackerböden durch zunehmend hohe Achslasten hat die Gefahr einer Unterbodenschädigung ansteigen lassen. Dies gilt vor allem für Böden mit Ľockerbodenwirtschaft (LBW) bei hohem Wassergehalt. Bodenbearbeitungssysteme wie die Festboden- Mulchwirtschaft (FMW), bei der zugunsten einer flachen Lockerung auf eine tief-wendende Bearbeitung verzichtet wird, können sich - im Vergleich zu gepflügten Böden - durch eine geringere Empfindlichkeit gegenüber mechanischer Belastung und ein höheres Regenerationsvermögen nach einer starken Belastung auszeichnen.

In der vorliegenden Arbeit wurde die Entwicklung von Böden mit FMW und LBW nach einer einmaligen mechanische Belastung über drei Jahre untersucht. Das Wachstum und der Ertrag von Sommergerste, Winterweizen und Wintergerste dienten als Indikator für belastungsabhängige Gefügeveränderungen durch die Belastung und über die Zeit.

In der Diskussion wird zunächst auf die Auswirkung des Bearbeitungssystems und der mechanischen Bodenbelastung auf bodenphysikalische Eigenschaften und auf die Bestandesentwicklung eingegangen. Abschließend werden aus den Erkenntnissen mögliche Konsequenzen für die pflanzenbauliche Praxis abgeleitet. In Übereinstimmung mit SOMMER et al. (1981) werden die Auswirkungen der mechanischen Belastung im ersten Versuchsjahr als Direktwirkung und in den Folgejahren als Nachwirkung bezeichnet.

5.1 Auswirkungen des Bearbeitungssystems und der mechanischen Bodenbelastung auf bodenphysikalische Eigenschaften

\subsubsection{Eindringwiderstand}

Der Widerstand, den ein Boden einer eindringenden Metallsonde entgegenbringt, beruht auf seiner mechanischen Festigkeit, auf der Verschiebbarkeit der Bodenteilchen. EHLERS et al. (1983a) konnten auf einer Parabraunerde aus Löß mit ca. $15 \%$ Tonanteil nachweisen, daß zunehmende Bodenfestigkeit gemessen als EDW - reduzierend auf das Wurzelwachstum wirkte. Für diesen Zusammenhang war der Bodenwassergehalt mit seiner Wirkung auf die Scherprozesse im Boden von großer Bedeutung. Der EDW soll in der vorliegenden 
Arbeit dazu dienen, Durchwurzelungswiderstände im Boden qualitativ zu erfassen.

Durch den Wendepflug werden im Bearbeitungshorizont kompakte, große Gefügeeinheiten aufgebrochen und als kleinere Gefügeelemente in lockerer Pakkung abgelegt. Der damit einhergehende starke Anstieg des Grobporenanteils führt zu einer Abnahme der Bodenfestigkeit und damit zu einem geringeren Widerstand des Bodens gegenüber der Verformung durch eine Metallsonde (COOTE \& RAMSEY 1983). Die Wirkung des Wendepfluges führte bei der Variante LBW 0t im Bearbeitungshorizont (0-25 cm Bodentiefe) auf den Standorten Reinshof und Relliehausen zu einem EDW von ca. 1,3 MPa (Abb. 7, 8 links). Auf einer Pseudogley Parabraunerde stellte FENNER (1995) im Bearbeitungshorizont des Wendepfluges bei einer Bodenfeuchte nahe der Feldkapazität (FK) einen EDW von ca. 0,6 MPa fest. Bei TEIWES (1988) wies der Bearbeitungshorizont einer Parabraunerde bei Pflugwirtschaft nahe der FK einen EDW von ca. 1,0 MPa auf. Unterhalb des Bearbeitungshorizontes, bei fehlender mechanischer Lockerung, war der EDW des Bodens bei LBW auf beiden Standorten deutlich höher als im Bearbeitungshorizont.

Auf dem Standort Reinshof wurden bei der Variante LBW 0t unterhalb des Bearbeitungshorizontes in der Krumenbasis - über die Versuchsjahre - Maximalwerte des EDW von ca.2,5 - 3,0 MPa gemessen. Auf dem Standort Relliehausen lagen die Maximalwerte dagegen höher bei 3,0 - 4,0 MPa. TEIWES (1988) und FENNER (1995) berichten von EDW in der Krumenbasis gepflügter Böden zwischen 3,0 und 6,0 MPa. Unterhalb der Verdichtungs- oder Verformungszone in der Krumenbasis nahm der EDW auf beiden Standorten bei der Variante LBW 0t, gemittelt über die Versuchsjahre, geringfügig ab. Dies entspricht den Erkenntnissen von TEIWES (1988) und FENNER (1995) auf Böden unter Pflug.

Die hohe Bodenbelastung führte im ersten Versuchsjahr auf dem gepflügten Boden des Standortes Reinshof zu einem drastischen Anstieg des EDW in Ober- und Unterboden. Dieses Ergebnis steht in Einklang mit den Untersuchungen von DANNOWSKI (1995): Auf einer Parabraunerde aus Geschiebemergel und Geschiebelehm mit ca. 7\% Tonanteil führte eine mechanische Bodenbelastung mit $100 \mathrm{kPa}$ Kontaktflächendruck zu einer Zunahme des EDW von ca. 2,0 auf 4,0 MPa in der Pflugsohle. Auch STEWART \& VYN (1994) berichteten von einem Anstieg des EDW in der Bodenschicht 12-35 cm bei einer Belastung 
mit 6t Radlast auf gepflügtem Boden. Ihre Untersuchungen fanden auf einer Tschernosem-Parabraunerde mit einem Tonanteil von ca. 24\% statt. Am Beispiel des Standortes Reinshof zeigt Abb. 51 die Beziehung von Lagerungsdichte und EDW in der Krumenbasis: In der Tendenz nahm bei LBW und FMW in den drei Versuchsjahren mit zunehmender Bodenbelastung die Lagerungsdichte und der EDW zu. Die steigende mechanische Belastung führte zu einer zunehmenden Erhöhung der Festigkeit und damit des Widerstandes des Bodens gegen eine Verformung.

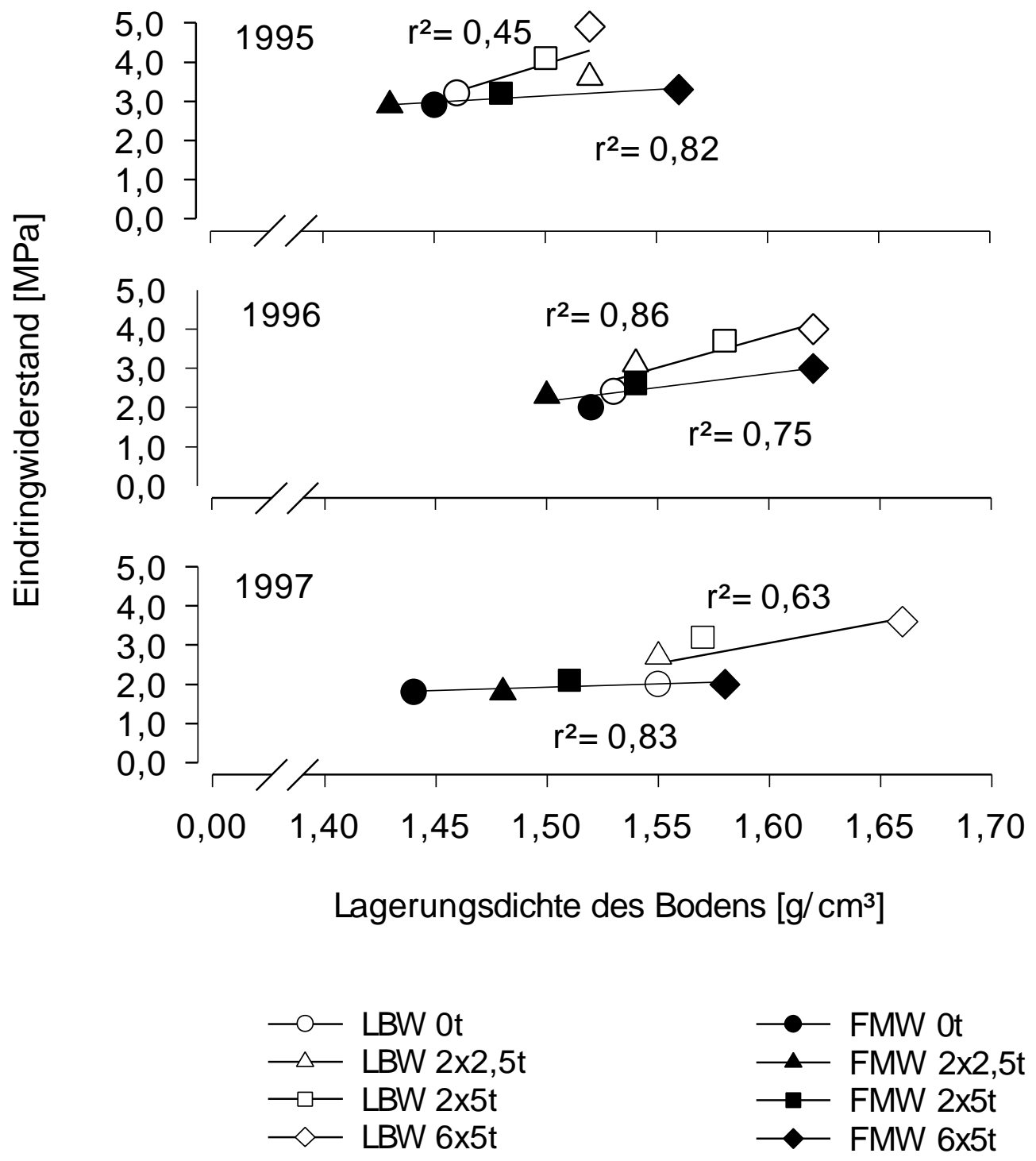

Abb. 51: Beziehung zwischen der Lagerungsdichte des Bodens und dem Eindringwiderstand (EDW) in der Bodentiefe 30-35 cm auf den Standort Reinshof. Die Daten zur Lagerungsdichte wurden nach Angaben der TLL, Jena zusammengestellt. 
Der Verlauf der EDW-Tiefenfunktionen bei LBW zeigt auf den Standorten Reinshof (Abb. 7 links) und Relliehausen (Abb. 8 links) die „Dauerhaftigkeit“ der Erhöhung des mechanischen Widerstandes in der Krumenbasis: Nach der mechanischen Bodenbelastung konnte kaum eine Abnahme des EDW in der Krumenbasis um $30 \mathrm{~cm}$ Bodentiefe festgestellt werden. Der durch die Nachwirkung der mechanischen Belastung langfristig erhöhte mechanische Widerstand in der Krumenbasis gepflügter Böden entspricht den Erkenntnissen von SCHJøNNING \& RASMUSSEN (1994), ETANA \& HÅKANSSON (1994) UND ARVIDSSON \& HÅKANSSON (1996).

Auf dem Boden mit FMW ohne zusätzliche Belastung zeigte der EDW auf dem Standort Reinshof unterhalb des Bearbeitungshorizontes, gemittelt über die Versuchsjahre, eine kontinuierliche Zunahme bei steigender Bodentiefe. In Relliehausen nahm der EDW bei FMW Ot unterhalb des Bearbeitungshorizontes bei zunehmender Bodentiefe geringfügig ab. Die gleichmäßige Veränderung von physikalischen Bodeneigenschaften ohne scharfe Brüche über das Bodenprofil scheint für reduziert bearbeitete Böden typisch zu sein (WENDROTH 1990, FENNER 1995, STOCKFISCH 1997). Durch die hohe Bodenbelastung nahm der EDW des Boden mit FMW beider Standorte nur in der Bodenschicht bis ca. 30 $\mathrm{cm}$ Bodentiefe zu. Unterhalb von $30 \mathrm{~cm}$ Bodentiefe änderte sich der EDW auch bei hoher Belastung kaum. Dieser Befund deutet darauf hin, daß bei FMW die hohe mechanische Belastung nur in den oberen Bodenschichten wirksam war. Verglichen mit dem gepflügten Boden war die „Tiefenwirksamkeit“ der mechanischen Belastung - gemessen am EDW - bei FMW wesentlich geringer. Die Beobachtung einer größeren Unempfindlichkeit von reduziert bearbeiteten Böden gegenüber mechanischer Belastung verglichen mit gepflügten Böden bestätigt die Erkenntnisse von BAEUMER \& PAPE (1972) sowie die Befunde von Hill \& MeZA-Montalvo (1990), EhLERS \& Claupein (1994) und Gruber (1994). Untersuchungen von WIERMANN (1998) auf dem Standort Reinshof zeigten, daß der Boden mit FMW im Vergleich zum gepflügten Boden unterhalb des Bearbeitungshorizontes eine mechanisch stabilere Aggregatstruktur hatte und deshalb ein höheres Kompensationsvermögen gegenüber mechanischer Belastung aufwies. 
Die EDW- Tiefenfunktionen des Standortes Reinshof zeigen, daß der mechanische Widerstand des Bodens unter $40 \mathrm{~cm}$ Bodentiefe auf dem Boden mit LBW und FMW bei allen Belastungsstufen $(2 \times 2,5 t$ und $2 \times 5 t$ sind nicht dargestellt) von 1995 bis 1997 kontinuierlich abgenommen hatte. Auffällig ist, daß die Reduktion des EDW über die Versuchsjahre bei beiden Bearbeitungssystemen und bei allen Belastungsstufen stattfand. Im folgenden sollen mögliche Gründe hierfür erörtert werden: Die Messungen des EDW wurden 1995 mit einem Penetrographen mit mechanischer Druckaufnahme durchgeführt. Möglicherweise wurde ein Teil der inneren Reibung des Gerätes erfaßt und führte 1995 zu einer Überschätzung des EDW bei allen Varianten. Trifft diese Vermutung zu, kann die Reduktion des EDW bei allen Varianten von 1995 nach 1996 ganz oder teilweise auf diesen systematischen Meßfehler zurückzuführen sein.

Die Ergebnisse von EHLERS et al. (1983a) und VOORHEES (1983) belegen die große Bedeutung des Bodenwassergehaltes bei der Ausprägung des mechanischen Widerstandes eines Bodens: Die Autoren zeigten auf, daß bei gleichbleibender Lagerungsdichte und einer Zunahme des Bodenwassergehaltes ein Absinken des EDW festzustellen ist. Dies kann nicht der Grund für die Reduktion des EDW von 1995 nach 1996 sein, da die Probenahmen in beiden Jahren bei annähernd gleichhohem Wassergehalt der Böden stattfanden. Möglicherweise führte aber der geringfügig höhere Bodenwassergehalt zur Probenahme 1997 verglichen mit 1996 - zu einer Reduktion des EDW in der Bodenschicht unter 40 $\mathrm{cm}$.

Eine weitere Ursache für die Reduktion des mechanischen Widerstandes eines Boden kann die Veränderung der Porengeometrie durch Frosteinwirkung sein: Gefrierendes Bodenwasser bildet Eislinsen und führt zu Frostsprengung (SCHEFFER \& SCHACHTSCHABEL 1984). Wie Abb. 52 zeigt, wurde weder im Winter 1995/96 noch im darauffolgenden Winter 1996/97 Bodenfrost in $50 \mathrm{~cm}$ Bodentiefe aufgezeichnet. Eine Reduktion des EDW unterhalb von $50 \mathrm{~cm}$ Bodentiefe durch Frosteinwirkung kann daher ausgeschlossen werden. Eine mögliche Frostwirkung in $20 \mathrm{~cm}$ Bodentiefe (Abb. 52 oben) wird bei LBW und FMW im Bearbeitungshorizont durch die Bodenbearbeitung überlagert. 


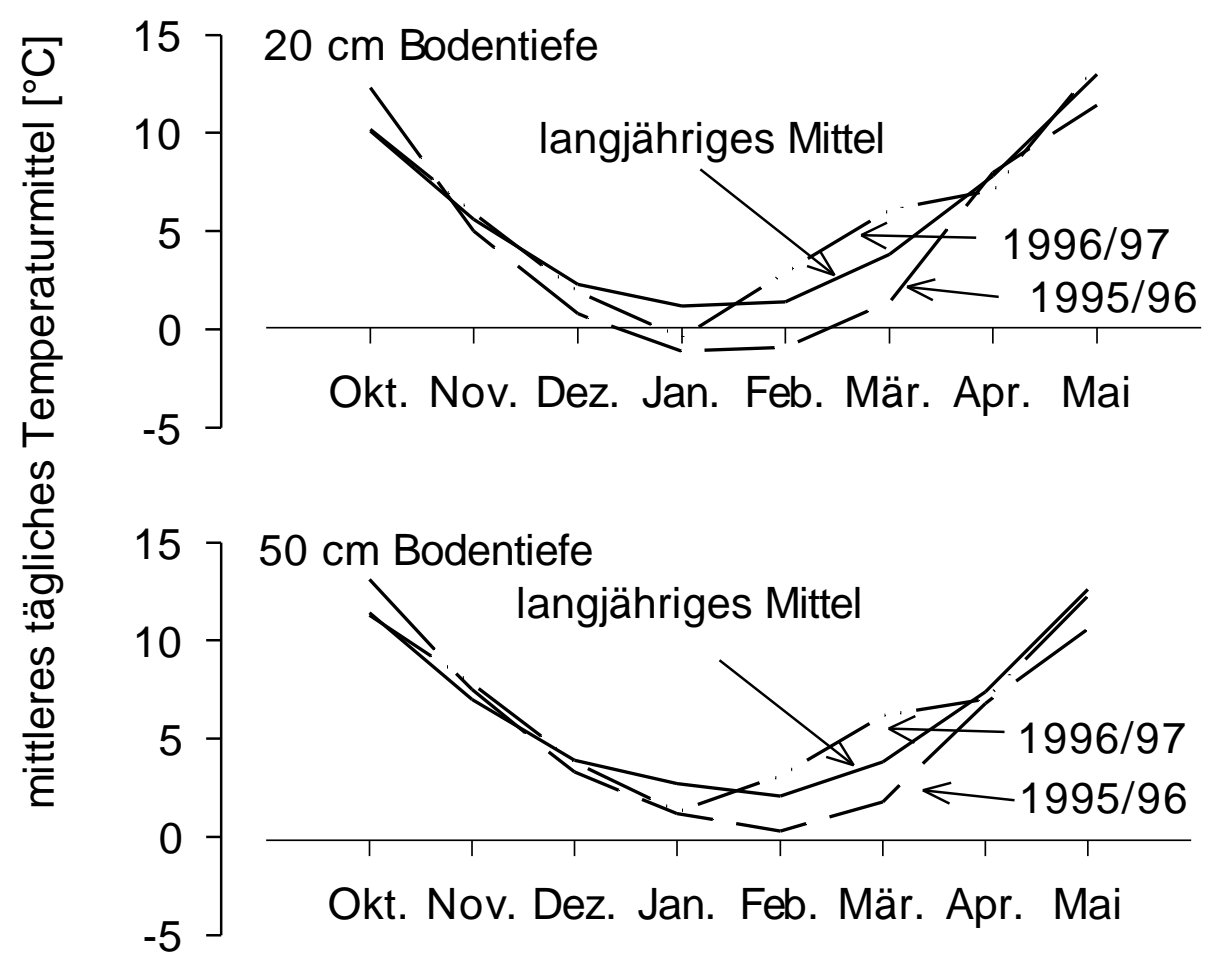

Abb. 52: Bodentemperatur in zwei Bodentiefen, gemessen an der Wetterstation Göttingen. Die Daten wurden zusammengestellt nach Angaben des Deutschen Wetterdienstes.

So wie in Reinshof (Abb. 7), so scheint auch in Relliehausen (Abb. 8) der EDW bei allen Varianten von 1996 nach 1997 abzunehmen. Möglicherweise ist auch auf dem Standort Relliehausen der geringere EDW 1997 mit einem höheren Wassergehalt des Bodens zur Probenahme zu erklären.

Wie schon bei den Arbeiten von VOORHEES (1983) und STEWART \& VYN (1994) stellte sich der EDW des Bodens als ein empfindlicher Indikator für die Wirkung einer mechanischen Bodenbelastung dar. Der EDW erfaßt die mechanische Festigkeit, den mechanischen Widerstand des Bodens. Aus der Literatur (TAYLOR et al. 1966, COCKROFT et al. 1969, EHLERS et. al. 1983a und DANNOWSKI 1995) ist die enge Beziehung von Bodenfestigkeit und Wurzelwachstum bekannt. Setzt man - vereinfachend - die Bodenfestigkeit gemessen als EDW des Bodens mit dem Widerstand gleich, den eine Pflanzenwurzel erfährt, so zeigte sich 1995 auf dem gepflügten Boden des Standortes Reinshof mit hoher Belastung ein über das gesamte Profil stark erhöhter Durchwurzelungswiderstand. Das entspricht den Erkenntnissen von DANNOWSKI (1995). Die Pflugarbeit 1996 und 1997 reduzierte den EDW in der Krume zwar erheblich. Im 
Unterboden, vor allem aber in der Krumenbasis, blieb der hohe Durchwurzelungswiderstand jedoch über die Versuchsjahre erhalten. Auf beiden Standorten wurde bei FMW eine Erhöhung des EDW - hervorgerufen durch mechanische Belastung - nur in der obersten Bodenschicht (0-30 cm Bodentiefe) gemessen. Auf dem Standort Großobringen zeigte der EDW als Funktion der Tiefe keine kennzeichnenden Unterschiede zwischen den Varianten und den Versuchsjahren (Abb. 9). Verglichen mit den Standorten Reinshof und Relliehausen zeichnet sich der Boden in Großobringen durch seinen hohen Tongehalt aus (Tab.7). WERNER (1996) weist darauf hin, daß die Tonpartikel des Bodens zur Probenahme (bei Frühjahrssättigung) möglicherweise vollständig von einer Wasserhülle umgeben waren. Der Boden war von der Metallsonde ohne deutlichen Reibungswiderstand zu durchdringen. Die Werte des EDW lagen über die gesamte Meßtiefe fast ausnahmslos zwischen 1,0 und 2,0 MPa (Abb. 9). Sowohl bei tiefer als auch bei flacher Bodenbearbeitung nahm die Lagerungsdichte des Bodens im ersten Versuchsjahr mit steigender Belastung zu (Tab. 47-49, Anhang). Dies deutete auf eine belastungsabhängige Gefügeveränderung hin, die nicht mit der Messung des EDW bei Frühjahrssättigung des Bodens erfaßt werden konnte.

\subsubsection{Transportfunktionen}

Ein leistungsfähiger Gasaustausch zwischen Boden und Atmosphäre ist für die Sauerstoffversorgung der Pflanzenwurzel (HILLEL 1980) sowie für den Abtransport wachstumshemmender Stoffwechselprodukten (BERGMANN 1954) und toxisch wirkender Gase von großer Bedeutung (BAEHR 1987, THORSTENSON \& POLLOCK 1989). Die Gasdiffusion im Boden findet fast ausschließlich im luftgefüllten Porenraum statt, da sich die wassergefüllten Poren durch einen wesentlich höheren Diffusionswiderstand auszeichnen. Kommt es infolge mechanischer Belastung zu einer Reduktion des luftführenden Porenraumes, kann der Gasaustausch zwischen Rhizosphäre und Atmosphäre und das Wurzelwachstum gehemmt werden. Die Porenraumgestaltung bedingt nicht nur die Gasdiffusion, sondern auch die Leitfähigkeit des Bodens für Wasser im ungesättigten Zustand. Vor allem die Grobporen und die Porenkontinuität spielen bei der Wasserleitung eine bedeutende Rolle (EHLERS \& TEIWES 1987, TEIWES 1988). Wird das Volumen der groben Poren oder die Porenkontinuität reduziert, kann 
eine drastische Reduktion der ungesättigten Wasserleitfähigkeit und damit eine Einschränkung der Wasserversorgung der Pflanzenwurzel die Folge sein (TEIWES 1988, FENNER 1995).

Auf den drei Standorten Reinshof, Relliehausen und Großobringen wurde auf den gepflügten Böden mit zunehmender mechanischer Bodenbelastung der Diffusionskoeffizient in der Bodenschicht 28-34 cm in der Krumenbasis deutlich eingeschränkt (Abb. 10-12 oben). Die Einschränkung der Diffusionskoeffizienten bei einer Wasserspannung von pF 2,0 ist - wie Abb. 53 am Beispiel Reinshof zeigt - eng korreliert mit der Zunahme des EDW des Bodens. Aus Abb. 51 (oben) geht hervor, daß die Zunahme des EDW mit der Zunahme der Lagerungsdichte verbunden war. Dies führt zu dem Schluß, daß die mechanische Belastung in der Bodenschicht 28-34 cm den Boden verfestigte und den Anteil grober Poren einschränkte (Tab. 41, Anhang). Die mit steigender Belastung zunehmende Porenverengung in der Krumenbasis führte zu einer Senkung der Diffusionskoeffizienten in dieser Bodenschicht. Die Befunde stehen in Einklang mit den Ergebnisse von CuRRIE (1960), McAFEE et al. (1989) und XU et al. (1992). In ihren Untersuchungen führte die mechanische Belastung von Böden unter Pflug ebenfalls zur Erhöhung der Lagerungsdichte, der Abnahme des Grobporenvolumens und zu einer Reduktion der Diffusionskoeffizienten.

Wie bei den Böden mit LBW zeigten sich auch bei den Böden mit FMW im ersten Versuchsjahr Gemeinsamkeiten der Standorte (Abb. 10,11 unten): Sowohl auf dem Standort Reinshof, als auch auf dem Standort Relliehausen konnte in der Tendenz bei steigender Bodenbelastung eine Zunahme der Lagerungsdichte bei gleichzeitiger Abnahme der Diffusionskoeffizienten bei pF 2,0 gemessen werden. Abb. 54 verdeutlicht die Abhängigkeit der Diffusionskoeffizienten bei pF 2,0 von der Belastungsstufe und dem Bearbeitungssystem: In Reinshof und Relliehausen lagen die Diffusionskoeffizienten auf dem flach bearbeiteten und gemulchten Boden bei steigender Belastung deutlich höher als auf dem gepflügten Boden. Dies läßt den Schluß zu, daß die Böden mit FMW auf beiden Standorten - unter dem Aspekt der Gasdiffusion - widerstandsfähiger gegen die mechanische Belastung waren als die Böden mit LBW. 
LBW

FMW

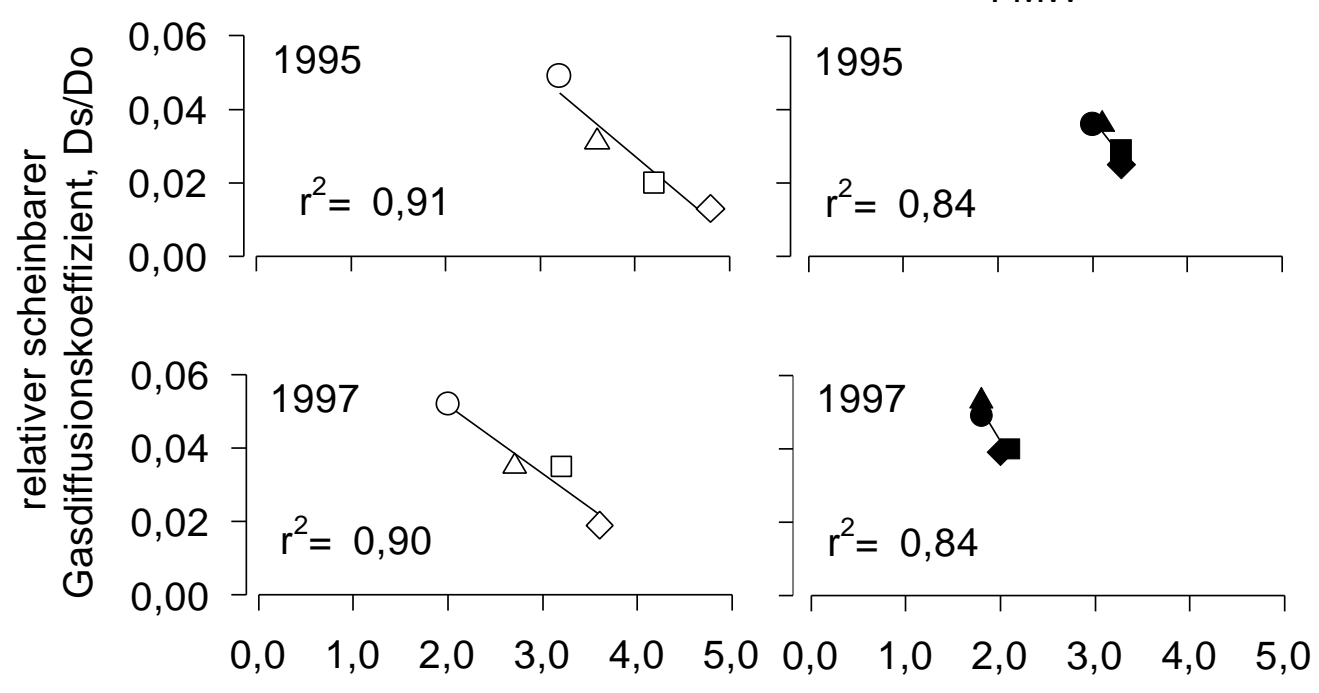

Endringwiderstand [MPa]

$$
\begin{aligned}
& \multimap-\text { LBW 0t } \\
& \square-\text { LBW 2x2,5t } \\
& \square-\text { LBW 2x5t } \\
& \neg \text { LBW 6x5t }
\end{aligned}
$$

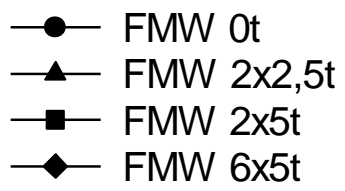

Abb. 53: Relativer scheinbarer Gasdiffusionskoeffizient bei einer Wasserspannung von pF 2,0 als Funktion des Eindringwiderstandes des Bodens in der Tiefe 28-34 cm auf dem Standort Reinshof in den Versuchsjahren 1995 und 1997.
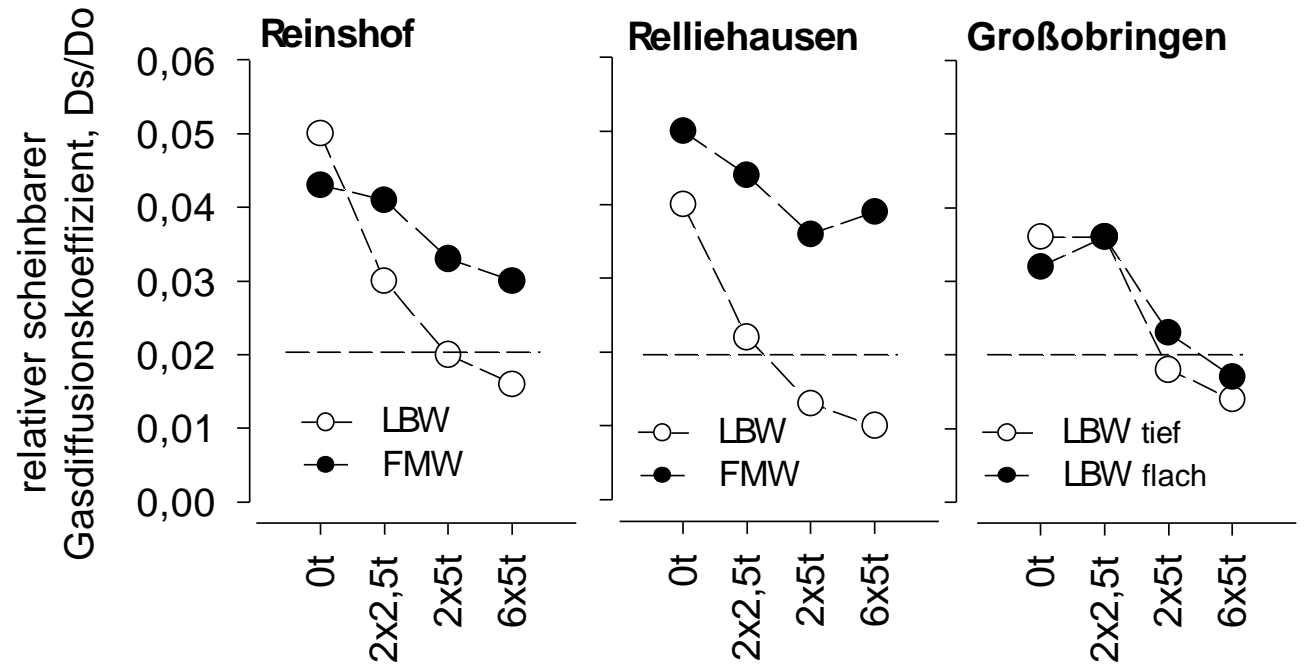

Überrollhäufigkeit x Radlast

Abb. 54: Relativer scheinbarer Gasdiffusionskoeffizient bei einer Wasserspannung von pF 2,0 in der Bodentiefe $28-34 \mathrm{~cm}$ in Abhängigkeit von der Belastungsstufe im Versuchsjahr 1995. Die gestrichelte, horizontale Hilfslinie markiert Ds/Do 0,02. Die Werte für Ds/Do wurden arithmetisch gemittelt. 
Bei der Betrachtung der Diffusionskoeffizienten zeigten sich standorttypische Unterschiede:

Der Standort Relliehausen zeichnete sich besonders durch seine hohe Vorbelastung aus. Der hohe Gehalt des Bodens an grobem Schluff und feinem Sand in Verbindung mit dem geringen Tongehalt (Tab. 6) führte zusammen mit hoher mechanischer Belastung durch regelmäßigen Zuckerrübenanbau in Verbindung mit hohen Niederschlagsmengen zu einem Kohärentgefüge mit geringer Gefügestabilität (FENNER 1995). Im Vergleich dazu zeichnete sich der Boden in Reinshof durch eine hohe Gefügestabilität aus (WERNER 1998). Abb. 54 zeigt, daß der ohnehin schon niedrige Diffusionskoeffizient bei LBW 0t in Relliehausen mit steigender Bodenbelastung erheblich tiefer sinkt als auf gepflügtem Boden in Reinshof.

Auf dem Standort Großobringen wurden zwei unterschiedliche Bearbeitungstiefen des Systems LBW verglichen. Die Ergebnisse deuten darauf hin, daß die Einschränkung der Diffusionskoeffizienten bei zunehmender mechanischer Belastung annähernd unabhängig von der Bearbeitungstiefe war (Abb. 54 rechts). Diese Befunde lassen in Übereinstimmung mit WIERMANN (1998) den Schluß zu, daß die reduzierte Bearbeitungstiefe bei wendender Bodenbearbeitung auf dem Standort Großobringen nicht zur Ausprägung eines „tragfähigeren“ Porensystems in der Bodenschicht 28-34 cm geführt hat.

Auf dem Standort Reinshof deutete sich bei gepflügtem Boden mit geringer, mittlerer und hoher Belastung eine Zunahme der Diffusionskoeffizienten bei pF 2,0 in 28-34 cm Bodentiefe von 1995 nach 1997 an (Abb. 55). Ebenso nahmen die Diffusionskoeffizienten bei FMW (alle Belastungsstufen) von 1995 nach 1997 zu (Abb. 55). Im Gegensatz zu LBW erreichten die Diffusionskoeffizienten bei FMW mit geringer, mittlerer und hoher Belastung das Niveau des unbelasteten Bodens. Diese Befunde deuten darauf hin, daß sich der Boden mit FMW im Vergleich zu LBW durch ein höheres Regenerationsvermögen auszeichnete. Der Grund für den Anstieg der Diffusionsrate kann bei beiden Systemen eine Zunahme des Diffusionsquerschnittes im wasserfreien Porenraum sein. Eine mechanische Lockerung kann nicht die Ursache sein, da bei beiden Bearbeitungssystemen die Untersuchungstiefe unterhalb des Bearbeitungshorizontes 
lag. WERNER (1998) schließt eine Auflockerung der verdichteten und verformten Bodenmatrix aus, weist aber auf folgenden Zusammenhang hin: Je stabiler ein Porensystem vor einer mechanischen Belastung ist, um so mehr Bioporen können den Deformationsprozess als isolierte Porenblase überstehen. Nach der Belastung können diese Porenräume durch Wurzelwachstum oder Wurmgänge wieder verbunden werden und ein System mit hoher Kontinuität ausbilden. Es ist denkbar, daß die Belastung isolierte Porenräume verursachte, die von 1995 bis 1997 wieder in ein neu entstandenes Porensystem aus Bioporen eingegliedert werden konnten. Möglicherweise hat auf dem Standort Reinshof allein die Aktivität der grabenden Makrofauna und das Wachstum der Pflanzenwurzeln eine Gefüge- und Porenraumveränderung des belasteten Bodens in der Bodentiefe 28-34 cm ausgelöst. Vor diesem Hintergrund erscheint das hohe Regenerationsvermögen bei FMW eng mit der intensiven Biodurchporung bei diesem Bearbeitungssystem verknüpft zu sein.

Wie Abb. 52 zeigt, war eine Neuorientierung von Bodenpartikeln in der Bodentiefe 28-34 cm durch Frosteinwirkung nicht oder nur in sehr geringem Umfang zu erwarten. Ein Einfluß des Wassers auf Gefüge und Porenraumgestaltung durch Quellung und Schrumpfung des Bodens kann auf Grund der geringe Änderung des Bodenwassergehaltes in der Bodentiefe unter $30 \mathrm{~cm}$ über den Versuchszeitraum wahrscheinlich ausgeschlossen werden. (Die Bodenwassergehalte sind nicht dargestellt.)

Wie aus den Abb. 14-16 hervorgeht, beeinträchtigte die hohe mechanische Belastung in Reinshof und Relliehausen bei LBW sowie in Großobringen bei LBW tief und LBW flach die ungesättigte hydraulische Leitfähigkeit im Meßbereich der Wasserspannung von 10 - ca. 50 hPa. Dies zeigt eine Einschränkung des groben Porenvolumens mit einem Äquivalentporendurchmesser von 60 bis $300 \mu \mathrm{m}$. Abb. 56 zeigt am Beispiel von Reinshof den eingeschränkten Beitrag der Poren mit einem Äquivalentdurchmesser von 60 bis $300 \mu \mathrm{m}$ an der ungesättigten hydraulischen Leitfähigkeit bei hoher Belastung. 


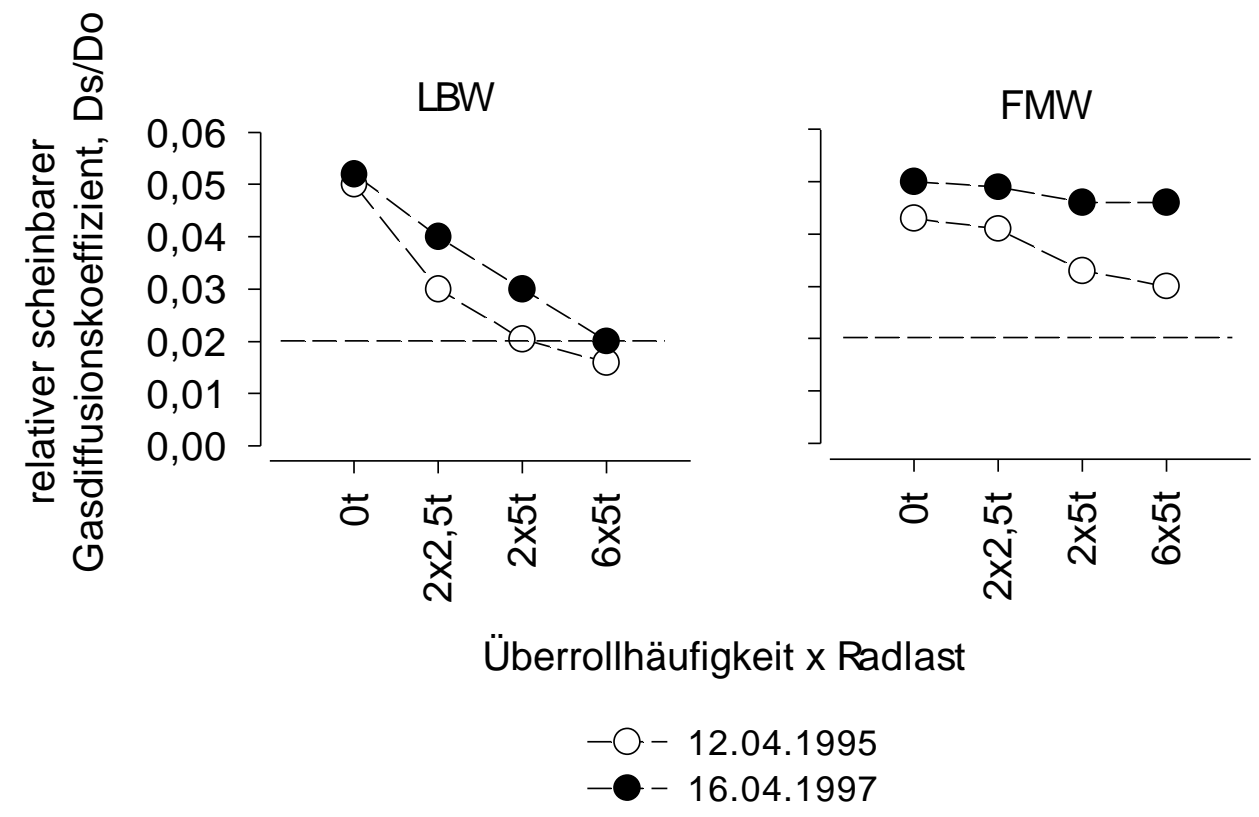

Abb. 55: Relativer scheinbarer Gasdiffusionskoeffizient bei einer Wasserspannung von pF 2,0 in der Bodentiefe $28-34 \mathrm{~cm}$ in Abhängigkeit von der Belastungsstufe in den Versuchsjahren 1995 und 1997 auf dem Standort Reinshof. Die gestrichelte, horizontale Hilfslinie markiert Ds/Do 0,02. Die Werte für Ds/Do wurden arithmetisch gemittelt.
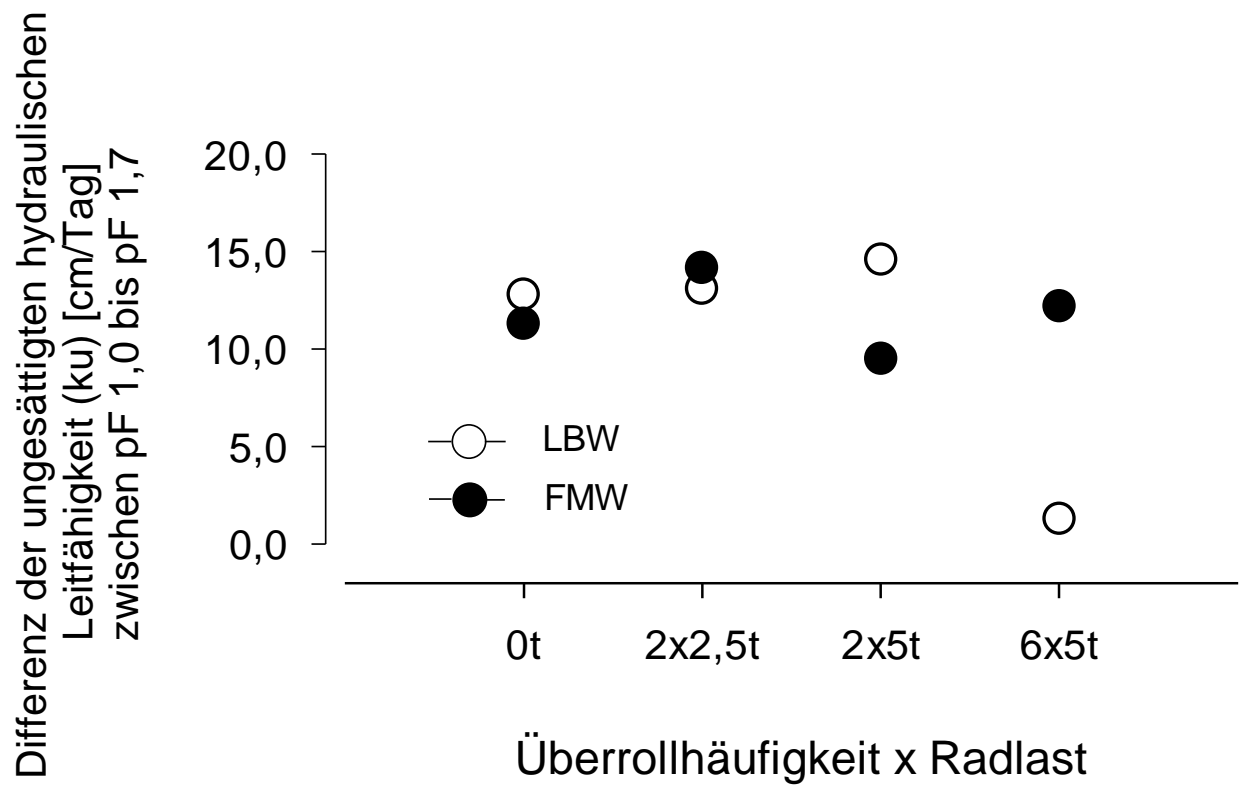

Überrollhäufigkeit x Radlast

Abb. 56: Beitrag der Poren von 60 bis $300 \mu \mathrm{m}$ Äquivalentdurchmesser an der ungesättigten hydraulischen Leitfähigkeit in Abhängigkeit von der Belastungsstufe auf dem Standort Reinshof in der Bodentiefe 28-34 cm am 12.04.1995. Dargestellt werden geometrische Mittelwerte. 
Sowohl in Reinshof als auch in Relliehausen wurde bei FMW keine Beeinträchtigung der ungesättigten hydraulischen Wasserleitfähigkeit gemessen (Abb. 14, 15 unten). Dies zeigt, in Übereinstimmung mit den Untersuchungen der Diffusionskoeffizienten, eine geringere Empfindlichkeit der Böden mit FMW gegen mechanische Belastung.

$\Rightarrow$ Auf den Standorten Reinshof und Relliehausen wurde durch die steigende mechanische Belastung im ersten Versuchsjahr bei LBW und FMW die Lagerungsdichte des Bodens in der Bodenschicht 28-34 cm erhöht und der Porenraum eingeschränkt. Dies führte vor allem bei LBW zu einem Anstieg des Eindringwiderstandes und zu einer Abnahme der Diffusionskoeffizienten sowie der ungesättigten hydraulischen Leitfähigkeit. Bei FMW sanken die Diffusionskoeffizienten bei steigender Belastung in der Bodenschicht 28-34 cm deutlich weniger als bei LBW. Beeinträchtigungen der ungesättigten hydraulischen Leitfähigkeit wurde bei FMW nicht festgestellt. Bezogen auf die Transportfunktionen für Luft und Wasser stellte sich der Boden mit FMW als weniger empfindlich gegenüber mechanischer Belastung dar als der Boden mit LBW.

Die belastungsbedingte Erhöhung des mechanischen Widerstandes und die Beeinträchtigung des Gasaustausches wurden in Reinshof bei LBW auch im dritten Versuchsjahr gemessen.

In Großobringen reagierte der Boden bei LBW mit tiefer Bearbeitung so, wie der Boden mit flacher Bearbeitung: Bei zunehmender Belastung stieg die Lagerungsdichte, der Porenraum wurde verengt und die Transportfunktionen eingeschränkt.

5.2 Auswirkungen der Bearbeitungssysteme und der mechanischen Bodenbelastung auf die Bestandesentwicklung

\subsubsection{Wurzelsystem}

Als Direktwirkung führte die mechanische Belastung auf den drei Standorten in der Tendenz zu einem Anstieg der Lagerungsdichte in der Bodentiefe 30-36 cm. (Tab. 41,44, 47, Anhang). Dies gilt in besonderem Maße für die Böden mit 
LBW. CZeratZKI (1972) wies darauf hin, daß bei einer Zunahme der Bodendichte das Wurzelwachstum durch die Erhöhung des mechanischen Widerstandes und die gleichzeitige Beeinträchtigung des Gasaustausches eingeschränkt wird. In Übereinstimmung mit diesen Befunden zeigt Abb. 53 einen Zusammenhang von EDW und Diffusionskoeffizienten: In Reinshof 1995 fielen - vor allem bei LBW - in der Krumenbasis die Diffusionskoeffizienten mit steigenden EDW stark ab. Aus Abb. 57 geht am Beispiel Reinshof 1995 hervor, daß die Wurzelsysteme bei LBW vermutlich sowohl von steigenden EDW, als auch von eingeschränktem Gasaustausch bei steigender Bodenbelastung beeinträchtigt wurden.

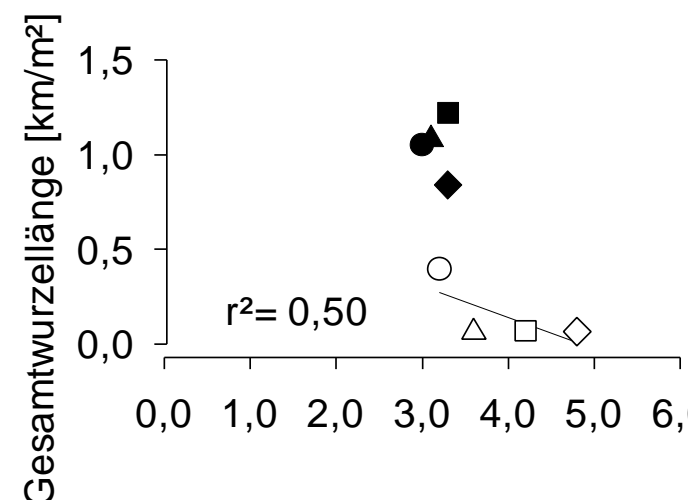

Endringwiderstand [MPa]

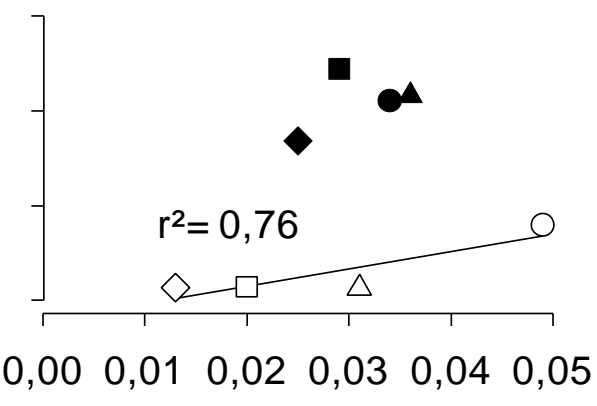

relativer scheinbarer Gasdiffusionskoeffizient, Ds/Do

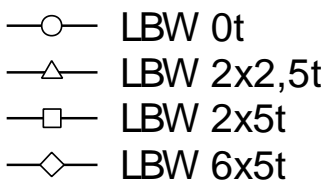

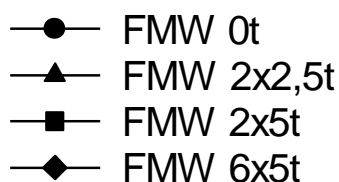

Abb. 57: Gesamtwurzellänge (GWL) unterhalb $35 \mathrm{~cm}$ Bodentiefe am 03.07.1995 als Funktion des Eindringwiderstandes in der Tiefe $30-35 \mathrm{~cm}$ am 04.04.1995 und des relativen scheinbaren Gasdiffusionskoeffizienten bei pF 2,0 in der Tiefe 28-34 cm am 12.04.1995 auf dem Standort Reinshof.

Die Ergebnisse lassen den Schluß zu, daß 1995 auf dem Boden mit LBW die Krumenbasis als Bodenschicht mit hohem EDW und eingeschränktem Gasaustausch vor allem bei den Belastungstufen gering, mittel und hoch als Durchwurzelungsbarriere wirksam wurde (Abb. 19, 21 links). Die mit zunehmender Bodenbelastung stark abnehmende Unterbodendurchwurzelung ist 
wahrscheinlich sowohl auf die gemeinsame Wirkung von hohen mechanischen Bodenwiderständen und beeinträchtigtem Gasaustausch als auch auf die im Vergleich zu den FMW - Stufen geringere Oberbodendurchwurzelung (Abb. 19, 21) zurückzuführen: Die mit steigender Belastung stark reduzierte Oberbodendurchwurzelung bedingte wahrscheinlich eine geringe Durchwurzelung des Unterbodens.

Beeinträchtigungen des Wurzelwachstums, verursacht durch eine hohe Lagerungsdichte oder einen hohen mechanischen Widerstand des Bodens, wurden bereits mehrfach beobachtet: In Laborversuchen konnten RUSSEL \& GosS (1974) und Goss (1977) nachweisen, daß das Wurzellängenwachstum von Getreide in einem Substrat aus Glaskugeln bei optimaler Versorgung mit Sauerstoff und Nährstoffen schon bei einer geringen Zunahme des mechanischen Widerstandes deutlich eingeschränkt wurde. Die Befunde legen den Schluß nahe, daß selbst bei optimaler Versorgung der Wurzel allein eine Zunahme des mechanischen Widerstandes als wachstumshemmender Faktor wirksam werden kann. ATWELL (1990) stellte bei Winterweizen in verdichtetem Boden ein verlangsamtes Wurzelwachstum fest, was zu einer unzureichenden ErschlieBung des Bodenprofils führte. In den Untersuchungen von LEHFELDT (1988) stellte sich bei Sommer- und Wintergerste eine negative Korrelation zwischen der Wurzellängendichte (WLD) und dem Grad der Bodenverdichtung dar.

Auf gepflügtem Boden ist beim Übergang vom Bearbeitungshorizont zum unbearbeiteten Unterboden auf Grund von Pflugsohlenverdichtungen in der Krumenbasis häufig mit einer Behinderung des Wurzelwachstums zu rechnen (BARRAClOUGH \& WeIR 1988, EhLERS et al. 1980/81, EHLERS 1983, EHLERS et al. 1986, AtWell 1990, OussiBle et al. 1992). Trifft eine Pflanzenwurzel auf eine Bodenschicht mit einer höheren Lagerungsdichte, einem reduzierten Grobporenanteil und eingeschränkten Transportfunktionen, so wird sie in ihrem Wachstum behindert: Ist der Durchmesser der Poren zu gering, um ein Durchwachsen zu ermöglichen, muß die Wurzel durch ein verstärktes Dickenwachstum Bodenpartikel verdrängen (MATERECHERA et al. 1991, MATERECHERA et al. 1992). Bei einem solchen Wachstum mit starker Zunahme des Wurzelquerschnittes kann das Längenwachstum eingeschränkt sein (BARLEY 1962, SCHUURMAN 1965, TAYLOR \& RATLIFF 1969, WiLSON et al. 1977, ATWELL 1990). Ist der mechanische Widerstand des Bodens höher als der Druck, den die Wur- 
zelspitze aufbringen kann, ist ein Eindringen nicht möglich, die Wurzel kann abgelenkt werden (PIETOLA 1991). Pflanzenwurzeln sind nicht in der Lage, ihren einmal erreichten Durchmesser zu reduzieren, um in eine kleinere Pore hineinwachsen zu können (WIERSUM 1957).

Anders stellten sich die Direktwirkung der mechanischen Belastung in Reinshof und Relliehausen bei FMW dar: Durch die Belastung stieg die Lagerungsdichte des Bodens in der Bodentiefe 28-34 cm bei den Stufen gering und mittel nur geringfügig an, deutlich stärker dagegen bei hoher Belastung (Tab. 41,44, Anhang, Abb. 51 oben). In Reinshof wurde im Vergleich zu LBW bei FMW der EDW in 28-34 cm Bodentiefe durch die Belastung kaum erhöht (Abb. 7, Abb. 51 oben). Der geringere EDW und der durch die Belastung deutlich weniger eingeschränkte Gasaustausch in der Bodenschicht 28-34 cm führte 1995 bei FMW im Vergleich zu LBW zu einer deutlich stärkeren Unterbodendurchwurzelung (Abb. 19). Am Beispiel Reinshof weist die Beziehung des relativen scheinbaren Gasdiffusionskoeffizienten zur Luftkapazität bei steigender Bodenbelastung (Abb. 58) auf eine mögliche Ursache hin.

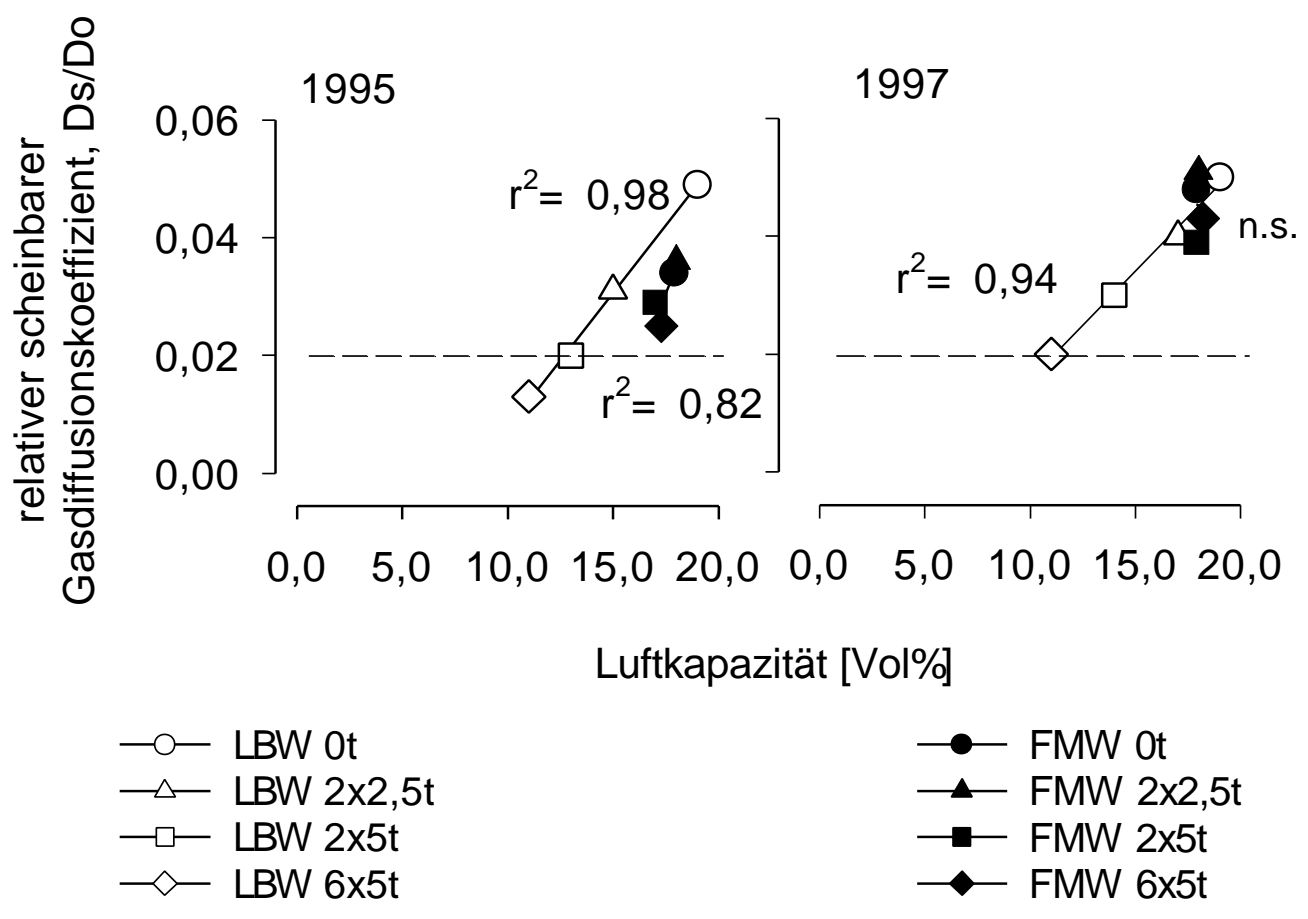

Abb. 58: Beziehung des relativen scheinbaren Gasdiffusionskoeffizienten bei $\mathrm{pF}$ 2,0 zur Luftkapazität ( $\mathrm{pF} 2,0)$ bei steigender Bodenbelastung in der Bodenschicht 28-34 cm auf dem Standort Reinshof am 12.04 .1995 und am 16.04.1997. Die gestrichelte Hilfslinie markiert Ds/Do 0,02. 
Bei LBW führte die geringe, mittlere und hohe Bodenbelastung in der Bodenschicht 28-34 cm zu einem deutlichen Verlust an groben Poren, verbunden mit einer starken Absenkung des Diffusionskoeffizienten. Im Gegensatz dazu wurde bei FMW in derselben Bodenschicht nur bei hoher Belastung der Porenraum deutlich eingeengt und die Diffusionskoeffizienten gesenkt. Offensichtlich konnten bei FMW - im Gegensatz zu LBW - Boden und Porensystem der geringen und mittleren Belastung standhalten, ohne erheblich in ihrer Funktion für das Wurzelwachstum beeinträchtigt zu werden (Abb. 57 rechts). Eine im Vergleich zu gepflügten Böden höhere Unempfindlichkeit gegenüber mechanischer Belastung bei reduziert bearbeiteten Böden steht in Einklang mit den Ergebnissen von DeXter (1988), BAUMGarTL (1991), DieZ (1991), EHLERS et al. (1994) und Gruber (1994). Aufgrund anhaltender Bodenruhe unterhalb des flachen Bearbeitungshorizontes verbunden mit einer hohen biologischen Aktivität konnte sich bei FMW in Relliehausen, vor allem aber in Reinshof ein stabiles Porensystem aus Wurmgängen und Kanälen abgestorbener Pflanzenwurzeln der Vorfrüchte bilden. Ein solches vornehmlich vertikal orientiertes Porensystem kann sich durch eine hohe Resistenz gegenüber einer vertikal auftreffenden Belastung auszeichnen (HARTGE \& BOHNE 1983, BLACKWELL et al. 1990). Es ist daher denkbar, daß bei FMW die geringe Belastungsstufe zu einer Stauchung, nicht aber zu einem drastischen Verlust von Bioporen geführt hat. Die mittlere und hohe Belastung führte bei FMW zu einem Verlust an Porenvolumen und zu einer Senkung der Diffusionskoeffizienten (Abb. 54).

Die durch die mechanische Belastung hervorgerufene Erhöhung der Lagerungsdichte, verbunden mit einer Zunahme des EDW und einer Beeinträchtigung des Gasaustausches schränkte in der Krumenbasis der gepflügten Böden die Wurzelsysteme der Sommergerste im Stadium EC 45 erheblich ein (Abb. 19 links). Abb. 59 zeigt am Beispiel Reinshof 1995 und 1997, wie die Pflanzenwurzeln im Verlauf der Vegetation beeinträchtigt wurden. Im ersten Versuchsjahr wurde bei LBW von EC 45 bis EC 85 bei den Belastungsstufen gering, mittel und hoch bei geringer Unterbodendurchwurzelung kaum eine weitere $\mathrm{Zu}$ nahme der Unterbodendurchwurzelung gemessen. Allein auf unbelastetem Boden nahm die Unterbodendurchwurzelung mit der Zeit zu (Abb. 59 und Tab. 50, 
51, Anhang). Dies legt den Schluß nahe, daß die erhebliche Beeinträchtigung der Porensysteme in ihrer Funktion für das Wurzelwachstum bei geringer, mittlerer und hoher Belastung auf dem gepflügten Boden über die Vegetation wirksam blieb. Ganz anders als bei LBW stellte sich die Wurzeldynamik in Abhängigkeit von der Belastungsstufe bei FMW (Abb. 59 links) dar: Bis EC 45 erreichte die Unterbodendurchwurzelung bei keiner, geringer und mittlerer und hoher Belastung ein wesentlich höheres Niveau als bei LBW. Von EC 45 bis EC 85 nahm die Unterbodendurchwurzelung deutlich ab. Eine Ausnahme bildete die hohe Belastung. Bei dieser Stufe nahm die Unterbodendurchwurzelung von EC 45 nach EC 85 zu (Abb. 59 links). Die im Vergleich zu LBW wesentlich höhere Unterbodendurchwurzelung bei FMW kann möglicherweise auf das ausgeprägt anisotrope Bioporensystem bei FMW, geschaffen von Pflanzenwurzeln und Regenwürmern, zurückgeführt werden.
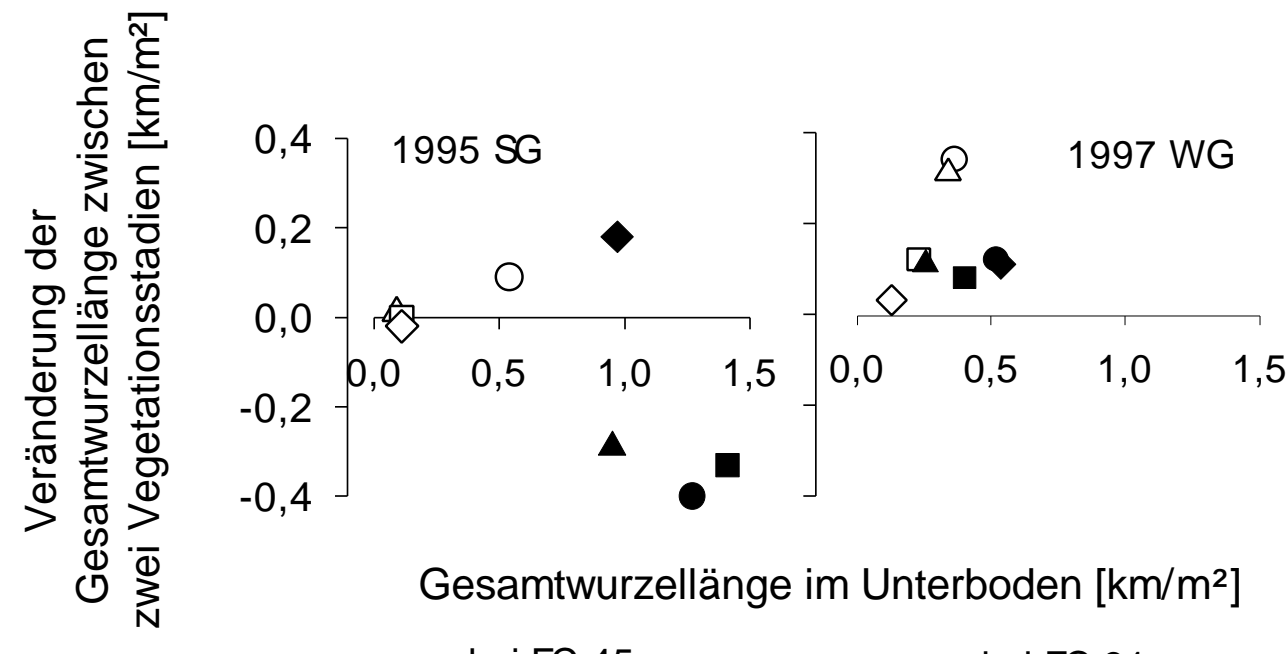

Gesamtwurzellänge im Unterboden $\left[\mathrm{km} / \mathrm{m}^{2}\right]$ bei EC 45 bei EC 31

$$
\begin{aligned}
& -\circ \text { LBW 0t } \\
& \triangle-\text { LBW } 2 \times 2,5 \mathrm{t} \\
& \rightarrow-\text { LBW } 2 \times 5 \mathrm{t} \\
& \multimap \text { LBW } 6 \times 5 \text { t }
\end{aligned}
$$

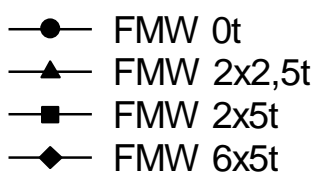

Abb. 59: Veränderung der Gesamtwurzellänge im Unterboden auf dem Standort Reinshof. Die Differenz der Gesamtwurzellänge ist für 1995 für den Abschnitt EC 45 bis EC 85, und 1997 für den Abschnitt EC 31 bis EC 45 dargestellt. Die Werte der Gesamtwurzellänge sind aus den Tab. 50, 51 und 55, 56, Anhang zu entnehmen. SG bezeichnet Sommergerste, WG Wintergerste. 
Im dritten Versuchsjahr zeigte sich auf dem gepflügten Boden bei mittlerer und hoher Belastung eine von EC 31 bis EC 45 anhaltende Beeinträchtigung der Unterbodendurchwurzelung (Abb. 59 rechts). Dies zeigt die deutliche Nachwirkung der Belastung im dritten Versuchsjahr. Bei FMW nahm die Unterbodendurchwurzelung von EC 31 bis EC 45 bei allen Belastungsstufen in gleichem Maße zu (Abb. 59 rechts), eine belastungsabhängige Beeinträchtigung wurde nicht gemessen.

Im Herbst 1995 brach die Pflugarbeit bei LBW den mechanisch belasteten Boden auf und lockerte den Oberboden für die Aussaat des Winterweizens (Tab. 57, Anhang). Die Wurzelsysteme des Winterweizens auf dem Standort Reinshof zeigten mit steigender Belastung eine Reduktion der GWL im Oberboden (Abb. 19 mitte). Dies legt den Schluß nahe, daß der belastete Boden - vor allem bei mittlerer und hoher Belastung - durch den Pflugeinsatz nicht optimal gelockert werden konnte.

Die trotz des hohen mechanischen Widerstandes (Abb. 7 links) auch bei hoher Belastung uneingeschränkte Durchwurzelung des Unterbodens zu EC 50 (Abb. 19 oben mitte) weist auf eine hohe Anpassungsfähigkeit des Winterweizens an Verdichtungserscheinungen im Unterboden hin. Auch bei einer Betrachtung der Unterbodendurchwurzelung über die Vegetation zeigten sich beim Winterweizen kaum belastungsabhängige Beeinträchtigungen (Tab. 52-54, Anhang). Allein die maximale Durchwurzelungstiefe nahm bei steigender Bodenbelastung ab (Abb. 19). Dies steht in Einklang mit den Befunden von BARRACLOUGH \& WEIR (1988). Die Autoren zeigten, daß Winterweizen auf einem Boden mit starker Pflugsohlenverdichtung im Vergleich zu Winterweizen ohne Pflugsohlenverdichtung dieselbe GWL, aber eine geringere maximale Durchwurzelungstiefe aufwies. In Reinshof wurde bei FMW 1996 wie schon 1995 nur bei hoher Belastung eine deutliche Zunahme der Lagerungsdichte verbunden mit dem Anstieg des mechanischen Bodenwiderstandes in der Bodenschicht 28-34 cm gemessen (Abb. 7 rechts, Tab. 42, Abb. 51). Diese Gefügeveränderung zeigte 1996 keine Auswirkungen auf das Wachstum der Weizenwurzeln (Abb. 19 unten mitte). Die im Vergleich zu Gerste größere Unempfindlichkeit des Winterweizens gegenüber Bodenverdichtungen ist aus der Literatur bekannt (SOMMER et al. 1995). Einen Hinweis auf den Prozess, der es der Weizenwurzel ermöglichte, den dicht gelagerten Boden zu durchdringen, gibt MERTEN (1998). Die Auto- 
rin wies nach, daß bei LBW unterhalb des Bearbeitungshorizontes in der Krumenbasis der mittlere Wurzelquerschnitt von Winterweizen mit steigender Belastung zunahm (Abb. 20 links). Dieser Befund steht in Einklang mit den Erkenntnissen von MATERECHERA et al. (1992). Die Autoren stellten dar, daß die Zunahme des Wurzeldurchmessers einen signifikanten Einfluß auf die Fähigkeit der Wurzeln hatte, dicht gelagerten Boden zu durchdringen. Als mögliche Ursache geben sie einen positiven Zusammenhang von Wurzeldurchmesser und Wurzeldruck an. Möglicherweise waren die Wurzeln des Winterweizens als Folge der Zunahme des Wurzelquerschnittes in der Lage, einen ausreichenden Druck zu erzeugen, um selbst bei hoher Bodenbelastung Bodenpartikel zu verdrängen und in den Unterboden vorzudringen.

Die Nachwirkung der mechanischen Belastung auf die Lagerungsdichte und den EDW des Bodens war in Reinshof und Relliehausen auch 1997 sowohl bei LBW als auch bei FMW nachzuweisen. Am Beispiel Reinshof zeigt Abb. 60, daß die Erhöhung des mechanischen Widerstandes des Bodens und die Beeinträchtigung des Gashaushaltes in der Krumenbasis des Bodens mit LBW auch noch bei der Wintergerste 1997 eine beträchtliche Durchwurzelungsbarriere darstellten. Es soll betont werden, daß die Durchwurzelung des Unterbodens in Reinshof bei LBW im dritten Versuchsjahr durch die hohe Bodenbelastung im Vergleich zu keiner Belastung um annähernd 80\% reduziert wurde (Abb. 19 rechts).

Wie ein Vergleich der Direktwirkung (Abb. 57) mit der Nachwirkung im letzten Versuchsjahr (Abb. 60) zeigte, hat die Einschränkung des Wurzelwachstums durch den hohen EDW und die Beeinträchtigung des Gashaushaltes bei LBW mit geringer und mittlerer und bei FMW mit hoher Belastung über die Zeit abgenommen.

Die Prozesse, die eine Veränderung des Porensystems und des Bodengefüges und damit ihrer Funktion für das Wurzelwachstum von 1995 bis 1997 verursacht haben könnten, wurden in Kap. 5.2.1 diskutiert. 


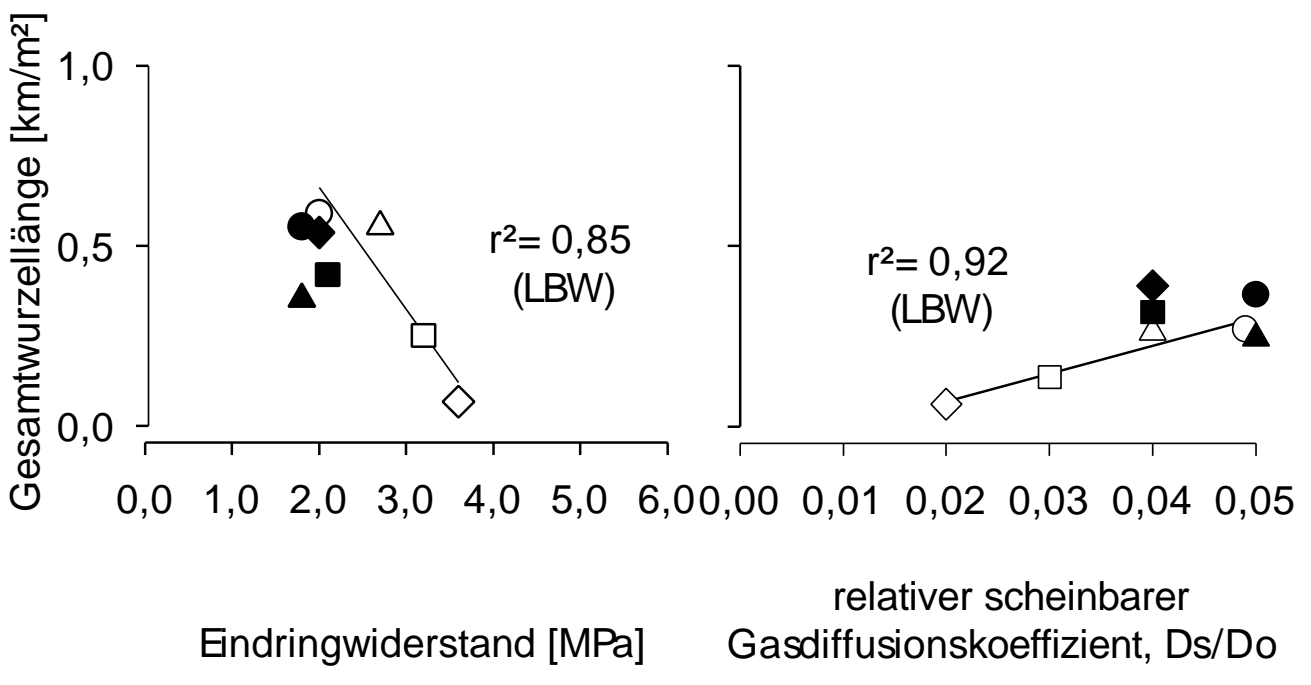

$$
\begin{aligned}
& \multimap-\text { LBW 0t } \\
& \multimap-\text { LBW } 2 \times 2,5 \mathrm{t} \\
& \square-\text { LBW } 2 \times 5 \mathrm{t} \\
& \multimap-\text { LBW } 6 \times 5 \mathrm{t}
\end{aligned}
$$

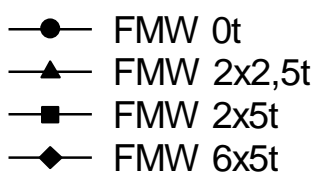

Abb. 60: Gesamtwurzellänge unterhalb $35 \mathrm{~cm}$ Bodentiefe am 02.06.1997 als Funktion des Eindringwiderstandes in der Tiefe 30-35 cm am 19.03.1997 und des relativen scheinbaren Gasdiffusionskoeffizienten bei pF 2,0 in der Tiefe 28$34 \mathrm{~cm}$ am 16.04.1997 auf dem Standort Reinshof.

Auf dem Standort Großobringen stellt sich die Direktwirkung der mechanischen Belastung ganz anders dar als auf den anderen Standorten (Abb. 22): Die bei steigender Bodenbelastung auftretende Einschränkung der Durchwurzelung des Unterbodens wurde bei beiden Bearbeitungstiefen von LBW durch eine stärkere Oberbodendurchwurzlung ausgeglichen, so daß bei allen Varianten die GWL in etwa auf gleicher Höhe lag. Im Gegensatz zu den Standorten Reinshof und Relliehausen war in Großobringen eine hemmende Direktwirkung der mechanischen Belastung auf das Wurzelwachstum im Oberboden nicht zu erkennen. Im Vergleich Reinshof und Relliehausen fand die Beprobung des Standortes Großobringen im ersten Versuchsjahr zu einem wesentlich späteren Zeitpunkt in der Vegetation statt . Es ist denkbar, daß das Wurzelwachstum im Oberboden bei steigender Bodenbelastung zu Beginn der Vegetation gehemmt war, dies aber durch verstärktes Wachstum im Laufe der Vegetation kompensiert wurde. Im Gegensatz dazu nahm bei LBW sowohl in Reinshof als auch in Relliehausen die Oberbodendurchwurzelung nur bei ho- 
her Bodenbelastung im Verlauf der Vegetation deutlich zu. Dagegen nahm auf nicht belastetem Boden die Oberbodendurchwurzelung im Verlauf der Zeit ab. BARRACLOUGH \& WEIR (1988) stellten bei Winterweizen zu Beginn der Vegetation im Frühjahr ein eingeschränktes Wurzelwachstum oberhalb einer verdichteten Bodenschicht fest. Im weiteren Verlauf der Vegetation bis EC 50 beobachteten die Autoren ein ausgleichendes Wachstum im Oberboden.

Auf eine weitere mögliche Ursache für die intensive Durchwurzelung des Oberbodens auf dem Standort Großobringen trotz mechanischer Belastung weist HORN (1998) hin: Auf Grund des hohen Tongehaltes des Bodens (Tab. 7) könnten sich schon direkt nach der Belastung bei einsetzender Trocknung und Schrumpfung des Oberbodens im frühen Sommer 1995 Risse im Boden gebildet haben, die der Sommergerste ein Durchwurzeln des Oberbodens ermöglichten.

Bei der Betrachtung des Standortes Großobringen ist zu bedenken, daß zur Pflugarbeit ein Spatenpflug eingesetzt wurden. Diese Art der Grundbodenbearbeitung erzeugt zwar - wie der Streichblechpflug - einen sprunghaften Übergang von lockerem gewendeten Boden im Bearbeitungshorizont zu ungelokkertem Boden unterhalb des Bearbeitungshorizontes. Ein Befahren der Pflugsohle und damit ein zusätzliches Verdichten und Verschmieren tritt jedoch nicht auf, da der Spatenpflug auf der Bodenoberfläche abgestützt wird (ESTLER 1996). Dennoch führte auch auf dem Standort Großobringen die steigende mechanische Belastung 1995 in der Bodenschicht 28-36 cm zu einem Anstieg der Lagerungsdichte (Tab. 47, Anhang) und zu einer Absenkung der Diffusionskoeffizienten des Bodens (Abb. 12), verbunden mit einer Einschränkung der Durchwurzelung des Unterbodens (Abb. 22 links).

In Großobringen wurde 1996 - etwas deutlicher als in Reinshof (Abb. 19 mitte oben) - auf gepflügtem Boden mit tiefer Bearbeitung eine Reduktion der Oberbodendurchwurzelung bei steigender Belastung gemessen (Abb. 22 mitte). Vermutlich führte der Pflugeinsatz im Herbst 1995 auf beiden Standorten nicht zu einem Aufbruch dichter Aggregate des mittel und hoch belasteten Bodens, die so wenig durchwurzelbar blieben. Im Gegensatz zu Reinshof nahm in Großobringen beim Winterweizen auch die Unterbodendurchwurzelung mit steigender Belastung ab. Möglicherweise war die geringere Unterbodendurchwurzelung eine Folge der ebenfalls geringen Oberbodendurchwurzlung. 
Ein weiterer Grund für die Einschränkung der Unterbodendurchwurzelung in Großobringen mag fehlende Regenwurmaktivität sein. Die grabende Tätigkeit von Regenwürmern kann eine förderliche Wirkung auf das Wurzelwachstum habe: Regenwurmgänge dienen den Pflanzenwurzeln als Leitbahnen und können ein Weg durch verdichtete Bodenschichten sein (ELLIS \& BARNES 1980, Ehlers et al. 1983, DeXTER 1986, Goss et al. 1988, MARTINO \& SHAYKEWICH 1994). LANGMAACK (1997) stellte auf dem Standort Großobringen eine außerordentlich geringe Regenwurmabundanz fest. Dies deutet darauf hin, daß der Winterweizen in Großobringen Bioporen zur Durchwurzelung nur in sehr geringem Umfang nutzen konnte.

Nach dem zweiten Pflugeinsatz im Herbst 1996 war in Großobringen bei beiden Bearbeitungstiefen von LBW eine Behinderung des Wurzelwachstums der Wintergerste im Unterboden zu erkennen. Dies zeigt, daß - so wie in Reinshof - auf dem Standort Großobringen die hemmende Wirkung der mechanischen Bodenbelastung auf das Wurzelwachstum bis ins dritte Versuchsjahr andauerte (Abb. 22).

\section{$\Rightarrow \quad$ Auf den Standorten Reinshof, Relliehausen und Großobringen} spiegelten die Wurzelsysteme die Direktwirkung der mechanischen Belastung auf Bodengefüge und Porenraumgestaltung deutlich wider: Steigende Eindringwiderstände verbunden mit einem beeinträchtigten Gashaushalt schränkten die Unterbodendurchwurzelung und die maximale Durchwurzelungstiefe mit steigender Belastung erheblich ein. Dies gilt besonders für die Böden mit LBW. Die durchwurzelungshemmende Wirkung der Pflugsohlenverdichtung in der Krumenbasis wurde durch die zunehmende mechanische Belastung stark erhöht. Auf Grund der im Vergleich zu LBW höheren Unempfindlichkeit des Bodens mit FMW gegenüber mechanischer Belastung wurden bei FMW die Funktionen des Bodengefüges und des Porensystems nur bei hoher Belastung eingeschränkt: die Wurzelsysteme wiesen nur bei hoher Belastung eine Beeinträchtigung der Unterbodendurchwurzelung auf.

Auf den Standorten Reinshof und Großobringen wurde die durchwurzelungshemmende Wirkung der mechanischen Belastung auf den gepflügten Böden bis ins dritte Versuchsjahr nachgewiesen. 


\subsubsection{Oberirdisches Sproßwachstum}

Das oberirdische Wachstum einer Getreidepflanze ist von der Entwicklung des Wurzelsystems nicht unabhängig. Wurzel und Sproß stehen in einem funktionellen Zusammenhang (DAVIDSON 1969, GREGORY 1994, PASSIOURA 1994, EHLERS 1996). Einschränkungen der Wurzel in ihrer spezifischen Funktion, Wasser- und Nährstoffe an den Sproß weiterzuleiten, können das Wachstum der oberirdischen Organe behindern. Ein beeinträchtigt wachsender Sproß mit geringer Blattfläche und eingeschränktem Stoffwechsel kann die Wurzeln möglicherweise nur unzureichend mit Assimilaten versorgen. Dies wiederum kann das Wurzelwachstum einschränken.

Im ersten Versuchsjahr zeichnete das oberirdische Sproßwachstum auf den Standorten Reinshof (Abb. 23, 24), Relliehausen (Abb. 31) und Großobringen (Tab. 23 ) die Direktwirkung der Belastung auf die Wurzelsysteme der Sommergerste deutlich nach. Bei LBW mit geringer, mittlerer und hoher Belastung wurde in Reinshof und Relliehausen bei stark reduzierter Durchwurzelung deutlich weniger Trockenmasse gebildet als bei keiner Belastung und nicht beeinträchtigter Durchwurzelung (Abb. 23, 31 links, Beispiel Reinshof Abb. 61). Bei FMW war in Reinshof die Durchwurzelung und die Sproßmasse nur bei hoher Belastung reduziert (Abb. 61). Hier zeigen sich deutliche Parallelen zur Beziehung von EDW, Diffusionskoeffizienten und GWL (Abb. 57).

Aus der Literatur ist bekannt, daß zwischen Wurzel- und Sproßausbildung eine Beziehung bestehen kann. SchuURMANN (1965) und ATWELL (1990) stellten fest, daß die Pflanzenhöhe bei Getreide auf verdichtetem Boden geringer ausfiel als auf unverdichtetem Boden. In Klimakammern stellten HofFMANN \& JUNGK (1995) bei Zuckerrüben eine Beeinträchtigung des Wurzelwachstums bei verdichtetem Boden fest. Mit steigender Reduktion der Wurzellänge nahm die Sproßmasse ab. Dieser Zusammenhang wurde von HOFFMANN (1993) auch bei Getreide nachgewiesen. In Laborversuchen konnten YouNG et al. (1997) erkennen, daß bei gleichbleibender Versorgung der Pflanzenwurzel mit Wasser und Nährstoffen allein eine Zunahme des mechanischen Bodenwiderstandes Wurzelwachstum und Blattlängenwachstum bei Weizen und Gerste deutlich reduzierte. 


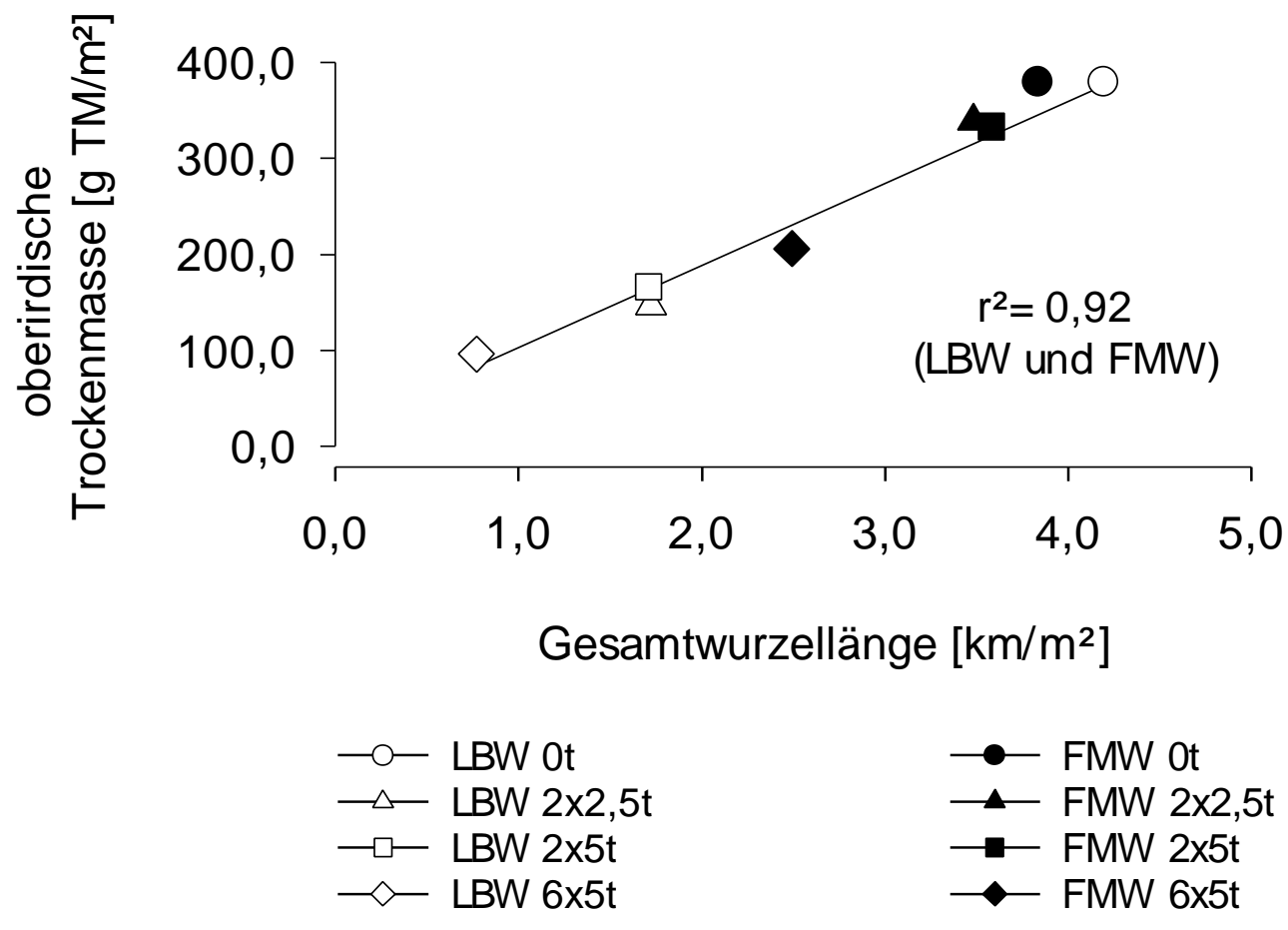

Abb. 61: Beziehung zwischen der Gesamtwurzellänge am 03.07.1995 (EC 45) und der oberirdischen Trockenmasse der Sommergerste am 23.06.1995 (EC37) auf dem Standort Reinshof.

Eine besondere Bedeutung kommt der Frage zu, auf welche Weise ein beeinträchtigtes Wurzelwachstum das Sproßwachstum beeinflußt. Möglicherweise kann eine eingeschränkte Nährstoff- und Wasserversorgung des Sprosses auf Grund der Reduktion des durchwurzelten Bodenraumes das oberirdische - aber auf Grund ihres funktionalen Zusammenhanges auch das unterirdische Wachstum beeinträchtigen. GEISLER \& RITZ (1981) dokumentierten an Sommerweizen in Wasserkultur eine Reduktion des Sproßwachstums bei vermindertem Nährstoffangebot im durchwurzelten Medium. Im Rahmen der vorliegenden Arbeit können jedoch keine Aussagen über die belastungsabhängige Nährstoffversorgung der Pflanzen gemacht werden. Die Bodenwassergehalte auf dem Standort Reinshof waren am 13.06.1995 bei beiden Bearbeitungssystemen und allen Belastungsstufen in der Bodenschicht von 10-100 cm Bodentiefe in etwa gleich hoch (ca. $31 \mathrm{Vol}$. \%). Die Bodenwassergehalte sind nicht dargestellt. Die Feldkapazität des Bodens lag in etwa bei 33 Vol.\%. Dies legt die Vermutung nahe, das im Frühsommer 1995 in Reinshof ein Wassermangel im Boden nicht vorlag. Da die Permeabilität der Pflanzenwurzel für Wasser bei 
eingeschränkter Sauerstoffversorgung gesenkt werden kann (EHLERS 1996), ist es denkbar, daß der erheblich eingeschränkte Gashaushalt bei LBW mit geringer, mittlerer und hoher Belastung und bei FMW mit hoher Belastung die Wasseraufnahme behindert haben könnte.

Im zweiten Versuchsjahr waren die Unterschiede in der Trockenmasse und im Blattflächenindex des Winterweizens zwischen den Belastungsstufen bei LBW und FMW auf den Standorten Reinshof und Relliehausen sehr gering. Für den Standort Reinshof überrascht diese Entwicklung nicht, da sich die Wurzelsysteme des Winterweizens trotz der Bodenbelastung annähernd uneingeschränkt entwickelten (Abb. 19 mitte oben). Bei FMW zeigt sich bei keiner Belastungsstufe eine Einschränkung des Wurzelsystems und auch keine Reduktion des Sproßwachstums.

Wie aus Abb. 19 (rechts oben) hervorgeht, war auf dem Standort Reinshof im dritten Versuchsjahr bei LBW das Wurzelsystem der Wintergerste im Unterboden bei hoher Bodenbelastung in der GWL und der maximalen Durchwurzelungstiefe deutlich reduziert. Dieser Mangel an Wurzelmasse und die im Vergleich zum unbelasteten Boden drastische Reduktion des durchwurzelten Bodens um ca. $80 \%$ spiegelte sich bei der Trockenmasse und dem BFI über die Vegetation wider (Abb. 28 links). Vor allem nach der Blüte lagen die Werte bei hoher Belastung deutlich unter denen der anderen Belastungsstufen. Möglicherweise zeigte sich hier ein Effekt der stark eingeschränkten Durchwurzelungstiefe bei hoher Belastung: Bei der Bereitstellung von Wasser und Nährstoffen konnten die Pflanzen nicht auf tiefere Bodenschichten zurückgreifen. Diese Mangelsituation könnte auch der Grund für das frühere Absterben der grünen Blattläche bei dieser Variante sein, welches sich in der geringeren Blattflächendauer (BFD) zwischen EC 69 und EC 87 ausdrückte (Abb. 29). Unterstützt wird diese Vermutung durch Untersuchungen von HSIAO (1993) und RAVICHANDRAN \& MUNGSE (1995). Die Autoren weisen darauf hin, daß die BFD von Weizen bei Wassermangel reduziert war. Bei FMW war - wie schon im zweiten Versuchsjahr bei Winterweizen - keine Beeinträchtigungen des Wurzelsystems (Abb. 19 rechts unten) oder des Sproßwachstums der Wintergerste (Abb. 28 rechts) zu erkennen. 
An dieser Stelle soll dem Zusammenwirken von Wurzel- und Sproßwachstum am Beispiel der Wintergerste auf dem Standort Reinshof besondere Aufmerksamkeit zukommen: Auf gepflügtem Boden wurde zum Zeitpunkt des Schossens bei hoher Bodenbelastung eine deutlich geringere GWL im Unterboden gemessen als auf dem Boden ohne Belastung (Abb. 19 rechts oben). Die GWL lag bei beiden Belastungsstufen im Oberboden auf einer Höhe (Abb. 19 rechts oben). Dies legt den Schluß nahe, daß das Wurzelsystem im Unterboden durch den hohen EDW und den beeinträchtigten Gashaushalt in der Krumenbasis (Abb. 60) beeinträchtigt wurde. Zur selben Zeit nahm die Bestandeshöhe auf dem Boden ohne Belastung schneller zu als auf dem Boden mit hoher Belastung (Abb. 30 links). Das eingeschränkte Wurzelsystem fiel mit einem beeinträchtigten Längenwachstum des Sprosses zusammen. Bei allen Belastungsstufen lag der Bodenwassergehalt in Ober- und Unterboden (nicht dargestellt) im Bereich der Feldkapazität. Im Frühjahr war bei allen Varianten eine NDüngung mit $70 \mathrm{~kg} \mathrm{~N} / \mathrm{ha}$ auf den bei LBW im Herbst gepflügten Boden ausgebracht worden (Tab. 57, Anhang). Es ist anzunehmen, daß die Versorgung der Pflanzen mit Wasser und Stickstoff im stark durchwurzelten Oberboden nicht eingeschränkt war. Dennoch trat eine Reduktion des Sproßlängenwachstums bei hoher Belastung ein.

Möglicherweise ist die Reduktion des Sproßlängenwachstums bei der Wintergerste - so wie bei der Sommergerste 1995 - auf einen Informationsaustausch zwischen Wurzel und Sproß zurückzuführen: Es ist denkbar, daß Wurzelspitzen, die in verdichtetem Boden auf hohen mechanischen Durchwurzelungswiderstand und einen eingeschränkten Gashaushalt stoßen, über hormonelle Steuerungsmechanismen das gesamte Wachstumsgeschehen der Pflanze beeinflussen können. Das gilt auch, wenn ein Teil des Wurzelsystems seine Funktion uneingeschränkt erfüllt. MASLE et al. (1990) und HOFFMANN (1993) dokumentierten ein beeinträchtigtes Sproßwachstum bei verdichtetem Boden schon kurz nach dem Auflaufen. Pflanzen in diesem Stadium haben zwar schon ein aktives Wurzelsystem. Dennoch findet die Ernährung hauptsächlich aus dem Endosperm statt (GEISLER 1983). Unter diesen Umständen kann eine Reduktion des Sproßwachstums nicht auf begrenzte Versorgung der Wurzel mit Wasser und Nährstoffen zurückgeführt werden. TARDIEU (1988) zeigte auf, daß das Wurzelwachstum von Mais bei beträchtlichen Spurverdichtungen nicht nur 
im verdichteten Boden unter der Spur, sondern auch im nicht verdichteten Boden neben der Spur beeinträchtigt war. MASLE \& PASSIOURA (1987) beobachteten bei Weizen auf einem verdichteten schluffigen Lehm in Klimakammern sowohl eine Beeinträchtigung des Wurzelwachstums als auch des Sproßwachstums. Weiterhin stellten AGUIRREZABAL et al. (1993) bei Mais neben der Beeinträchtigung der Wurzeln in verdichtetem Boden eine Reduktion des Wachstums sproßbürtiger Wurzeln außerhalb des verdichteten Bodens fest. TARDIEU (1994) beobachtete den beschränkenden Effekt einer Bodenverdichtung auf das Wurzel- und Sproßwachstum und nahm eine hormonelle Interaktion von Wurzel und Sproß als eine Ursache an. Aus der Literatur geht hervor, daß die Wirkung wachstumshemmender Bodenbedingungen auf den Hormonhaushalt der Pflanzen wahrscheinlich ist, aber noch nicht komplett aufgeklärt werden konnte (Kays et al. 1974, Torrey 1976, Campell \& Moreau 1979, Carmi \& Heuer 1981, LACHNO et al. 1982, MASLE \& PASSIOURA 1987, DAVIES \& ZANG 1991, TARDieu 1994, Coleman \& SCHNeIDER 1996, MullHolland et al. 1996, MunNS \& Cramer 1996, ZHANG \& TARDIEU 1996).

In Großobringen führte die Direktwirkung der Belastung 1995 auf gepflügtem Boden - so, wie es sich auch in Reinshof 1995 andeutete (Abb. 24 links) - zu einer Reduktion der Bestandeshöhe auf gepflügtem Boden (Tab. 23). Dies zeigte eine deutliche Parallele zur belastungsabhängigen Reduktion der Diffusionskoeffizienten (Abb. 12) und der Unterbodendurchwurzelung (Abb. 22 links): Unabhängig von der Bearbeitungstiefe nahm die Bestandeshöhe bei LBW mit steigender Belastung ab. Die Beeinträchtigung der Wurzelsysteme fiel wie in Reinshof mit einer Reduktion des oberirdischen Sproßwachstums zusammen. Im Gegensatz zur Direktwirkung hatte die Bearbeitungstiefe in den Versuchsjahren 1996 und 1997 in Großobringen - unabhängig von der Belastungsstufe - einen Einfluß auf die Bestandeshöhe: Tendenziell lag die Bestandeshöhe bei tiefer Bodenbearbeitung höher als bei flacher Bearbeitung (Tab. 24, 25). Bei LBW tief wurde der Boden um ca. $12 \mathrm{~cm}$ tiefer gelockert als bei LBW flach. Dadurch stand den Pflanzen im Vergleich zu LBW flach zu Beginn ihrer Entwicklung ein größeres Bodenvolumen mit günstiger Lockerung zu Verfügung. Möglicherweise führte dies in Großobringen zu Wachstumsbedingungen zur Zeit des Schossens, welche eine größere Wachstumshöhe zur Folge hatten. 
$\Rightarrow$ In Reinshof und Relliehausen wurde 1995 bei stark reduzierter Durchwurzelung eine verminderte Bildung von oberirdischer Sproßmasse gemessen. So wie bei der Sommergerste 1995 wurde dieser Zusammenhang in Reinshof auf gepflügtem Boden auch bei der Wintergerste im Jahr 1997 nachgewiesen. Möglicherweise war eine eingeschränkte Versorgung von Wurzel und Sproß durch die stark begrenzte Profilerschließung eine Ursache. Zusätzlich ist eine beeinträchtigte Wasseraufnahme der Wurzel, verursacht durch den eingeschränkten Gashaushalt denkbar.

\subsection{3 $\mathrm{CO}_{2} / \mathrm{H}_{2} \mathrm{O}$ Gaswechsel}

Sowohl beim Winterweizen als auch bei der Wintergerste zeigten die Messungen des Gesamtwasserpotentials und des $\mathrm{CO}_{2} / \mathrm{H}_{2} \mathrm{O}$ Gaswechsel der Fahnenblätter auf gepflügtem Boden einen Zusammenhang: Tendenziell lag bei beiden Feldfrüchten die Assimilationsrate (Abb. 38, 44 links) und das Gesamtwasserpotential (Abb. 39, 45 links) über den Tag bei hoher Bodenbelastung niedriger als auf nicht belastetem Boden. $\mathrm{CO}_{2}$ - Assimilationsrate und stomatäre Leitfähigkeit sind über den Öffnungszustand der Stomata eng miteinander verknüpft und zeichnen sich durch eine positive Korrelation aus (LARCHER 1984, BETHENOD et al. 1996). Der Öffnungszustand der Stomata ist stark abhängig vom Gesamtwasserpotential (GWP) in der Pflanze. Fällt das GWP unter einen Schwellenwert, verengen sich die Stomata. Bei weiter abfallendem GWP kommt es zu einem Spaltenschluß (LARCHER 1984). Ein niedriges GWP kann ursächlich mit einem in seiner Größe oder in seiner Funktion beeinträchtigten Wurzelsystem zusammenhängen: Verliert die Pflanze mehr Wasser durch Transpiration als sie durch das eingeschränkte Wurzelsystem aufnehmen kann, fällt das GWP. Auf dem Standort Reinshof unterschied sich bei LBW 1996 die Unterbodendurchwurzelung des Winterweizens bei keiner und hoher Belastung kaum (Abb. 19 mitte oben). Das bei hoher Belastung niedrigere GWP (Abb. 39 links) ist daher 1996 nicht mit einer eingeschränkten Wasseraufnahme durch ein reduziertes Wurzelsystems im Unterboden zu erklären. Der prozentuale Anteil wassergefüllter Poren am Gesamtporenvolumen in der Bodentiefe 30$100 \mathrm{~cm}$ war am 16.06.1996 bei LBW 6x5t (61,9\%) deutlich höher als bei LBW 
Ot $(51,0 \%)$. Ebenso war bei hoher Belastung die Luftkapazität des Bodens in der Bodenschicht 30-50 cm deutlich geringer als bei keiner Belastung (Tab. 42, Anhang). Diese Befunde legen den Schluß nahe, daß der Gashaushalt und damit die Sauerstoffversorgung der Wurzeln am 16.06.1996 bei hoher Belastung eingeschränkt war. Möglicherweise führte dies zu einer Beeinträchtigung der Wurzeln in ihrer Funktion, Wasser aufzunehmen und damit - unter den Transpirationsbedingungen vom 23.06.1996 - zu einem im Vergleich zur Kontrolle (LBW 0t) stärker gefallenem GWP.

Im Gegensatz zu LBW (Abb. 39 links) fiel das GWP bei FMW (Abb. 39 rechts) am 23.06.1996 über den Tagesgang weniger tief ab und zeigte kaum Unterschiede zwischen keiner und hoher Belastung. Das höhere GWP bei FMW führte jedoch nicht zu einer höheren $\mathrm{CO}_{2}$-Assimilation. Da bei annähernd gleicher GWL der Unterbodendurchwurzelung das GWP bei FMW weniger tief abfiel als bei LBW, liegt die Vermutung nahe, daß das Wurzelsystem im Unterboden bei FMW im Vergleich zu LBW nicht oder weniger in seiner Funktion Wasser aufzunehmen eingeschränkt war.

Wie beim Winterweizen, so war auch bei der Wintergerste bei LBW und hoher Bodenbelastung die stomatäre Leitfähigkeit, die Assimilationsrate der Fahnenblätter und das Gesamtwasserpotential über den Tag tendenziell niedriger als auf dem Boden ohne Belastung (Abb. 44 links). Im Gegensatz zum Weizen war bei der Gerste die Reduktion des Wurzelsystems im Unterboden bei hoher Belastung wesentlich deutlicher (Abb. 19). Abb. 62 zeigt die Beziehung von Gesamtwurzellänge im Unterboden und stomatärer Leitfähigkeit. Zum Zeitpunkt der Gaswechselmessungen war der pflanzenverfügbare Bodenwassergehalt bei hoher Belastung in den ersten $30 \mathrm{~cm}$ Bodentiefe annähernd ausgeschöpft: Ohne Bodenbelastung lagen die Werte nahe des PWP (Bodenwassergehalte sind nicht dargestellt). Unterhalb von $30 \mathrm{~cm}$ Bodentiefe war der Wassergehalt des Bodens bei beiden Belastungsstufen auf gleicher Höhe. Pflanzenverfügbares Wasser stand zur Verfügung. Bei hoher Bodenbelastung konnten die Pflanzen aus dem Oberboden kein Wasser mehr aufnehmen, da der Vorrat erschöpft war. Unterhalb von ca. $50 \mathrm{~cm}$ Bodentiefe konnten die Pflanzen wahrscheinlich ebenfalls kein Wasser aufnehmen, da in dieser Tiefe keine Wurzeln vorhanden waren (Abb. 19 rechts oben). Diese Beeinträchtigung der Wasseraufnahme der 
Wintergerste bei hoher Bodenbelastung kann als Ursache für das niedrige GWP und den eingeschränkten Gaswechsel angesehen werden.

Bei der Wintergerste 1997 zeigte sich auf dem Boden mit FMW und den Belastungsstufen 0t und 6x5t eine unerwarteter Zusammenhang: Bei annähernd gleicher Ausprägung des Wurzelsystems (Abb. 19 rechts unten) lag die stomatäre Leitfähigkeit und die $\mathrm{CO}_{2}$-Assimilationsrate (Abb. 44 rechts) der Fahnenblätter, sowie das GWP der Pflanzen ( Abb. 45 unten) bei hoher Belastung in der Tendenz höher als auf unbelastetem Boden. Der Bodenwassergehalt zum Zeitpunkt der Messung des GWP unterschied sich nicht zwischen den Belastungsstufen (nicht dargestellt). Eine begründete Erklärung für dieses Phänomen ist nicht gefunden worden.
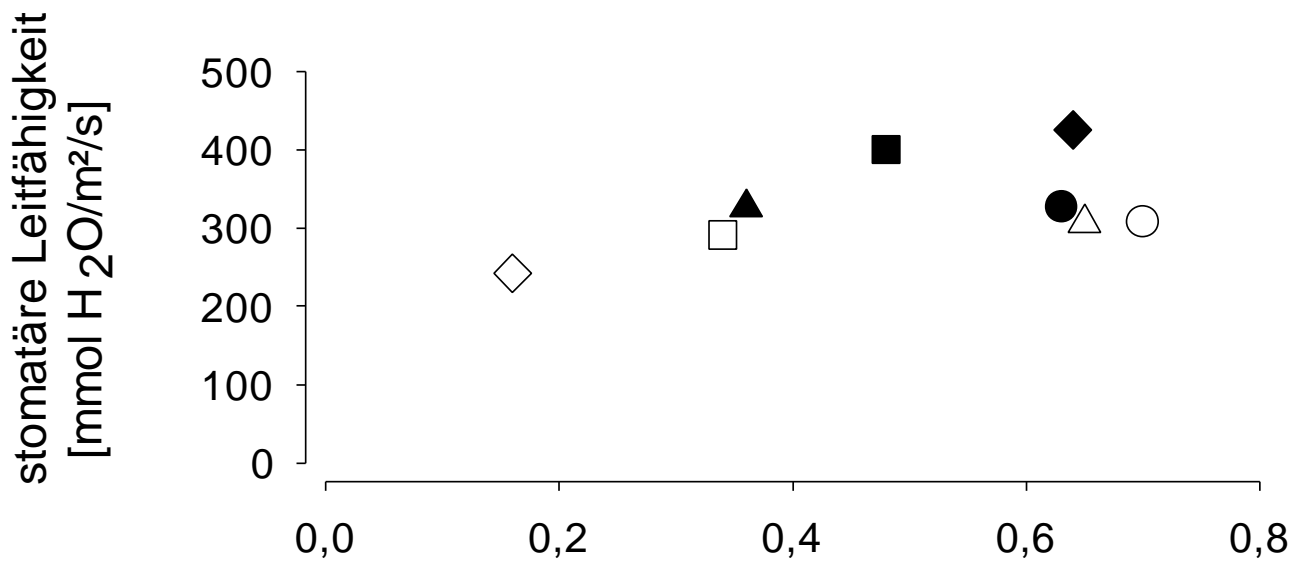

Gesamtwurzellänge im Unterboden $\left[\mathrm{km} / \mathrm{m}^{2}\right]$

$$
\begin{aligned}
& \multimap-\text { LBW 0t } \\
& \neg-\text { LBW 2x2,5t } \\
& \square-\text { LBW } 2 \times 5 \mathrm{t} \\
& \multimap \text { LBW 6x5t }
\end{aligned}
$$

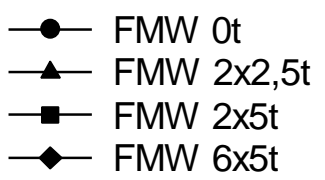

Abb. 62: Beziehung zwischen der Gesamtwurzellänge im Unterboden am 02.06.1997 und der mittleren stomatären Leitfähigkeit am 18. und 19.06.1997 auf dem Standort Reinshof für Wintergerste.

BETHENOD et al. (1996) wiesen darauf hin, daß alle Prozesse, welche die $\mathrm{CO}_{2}^{-}$ Assimilation und den Öffnungszustand der Stomata betreffen, über Regelkreise eng miteinander verknüpft sind. Innerhalb dieser Regelkreise spielt die chemische Signalübermittlung eine bedeutende Rolle. DöRFFLING (1981) berichtete 
von einem Stomataschluß beim Anstieg der Abscisinsäurekonzentration im Blatt und beschrieb eine Zunahme der Abscisinsäurekonzentration bei zunehmendem Wassermangel im Boden. MASLE \& PASSIOURA (1987) dokumentierten die beeinträchtigenden Einflüsse einer Bodenverdichtung auf die stomatäre Leitfähigkeit bei Weizen. Die Autoren vermuteten, daß das Sproßwachstum und der Gaswechsel bei verdichtetem Boden hauptsächlich durch hormonale Signale aus der Wurzel beeinflußt wurden. DAVIES \& ZHANG (1991) und TARDIEU (1994) betonten, daß ein hoher mechanischer Widerstand des Bodens über eine chemische Signalübermittlung aus der Wurzel eine Begrenzung der stomatären Leitfähigkeit hervorrufen kann. Es ist deshalb anzunehmen, daß auch im vorliegenden Fall bei der Regelung des Gaswechsels der Fahnenblätter und des GWP unter Streßbedingungen Hormone eine zentrale Rolle spielten.

Im Vergleich der Lichtfluß-Effektkurven (Abb. 42, 47) stellte sich heraus, daß auf gepflügtem Boden sowohl beim Winterweizen als auch bei der Wintergerste bei gleicher Strahlungsmenge auf dem hoch belasteten Boden deutlich weniger $\mathrm{CO}_{2}$ assimiliert werden konnte als bei keiner, geringer und mittlerer Belastung. In Übereinstimmung mit diesem Ergebnis stellten BETHENOD et al. (1996) bei Mais auf einem verdichteten tonigen Lehm eine eingeschränkte $\mathrm{CO}_{2}$ Assimilation über einen weiten Bereich der eingestrahlten Lichtmenge fest. Als Ursachen sind die eingeschränkte stomatäre Leitfähigkeit bei Wasser- und Nährstoffmangel, sowie hormonelle Interaktionen zwischen Sproß und Wurzelsystem wahrscheinlich.

Diese Befunde zeigen, daß die mechanische Bodenbelastung auf gepflügtem Boden noch zwei Jahre nach dem Lasteintrag über den Einfluß auf die Wurzelsysteme hemmend auf den Gaswechsel der Wintergerste wirken konnte (Abb. $62)$.

Die Gaswechselmessungen erfassen zeitlich einen nur sehr geringen Ausschnitt der Vegetation und deuten einen Zusammenhang von Bodenverdichtung und Gaswechsel nur an. Um umfassendere Aussagen über den Effekt eines beeinträchtigten Wurzelwachstums auf die $\mathrm{CO}_{2}$-Assimilation machen zu können, sollten längere Zeitabschnitte bei einem hohen Sättigungsdefizit der umgebenden Luft und annähernd erschöpftem Bodenwasservorrat durchgeführt werden. Da die beschriebenen Bedingungen bei Feldversuchen stark von der 
längerfristigen Witterung abhängen, sind Laborversuche möglicherweise geeigneter als Untersuchungen im Feld, da die Wachstumsbedingungen besser kontrollierbar sind. Andererseits sind Bodenverdichtungen unter Laborbedingungen nur schwer zu simulieren.

Bei der Betrachtung der $\mathrm{CO}_{2}$-Assimilationsraten und der Lichtfluß-Effektkurven muß berücksichtigt werden, daß in der vorliegenden Arbeit nur das Fahnenblatt der Getreidepflanzen Gegenstand der Untersuchungen war. THORNE (1965) wies darauf hin, daß die grüne Fläche der Ähre bei unbegranntem Weizen ca. 40\% der Fläche der Fahnenblattes erreichen kann. Bei begrannter Gerste kann die grüne Fläche der Ähre im Verhältnis zur Fläche des Fahnenblattes noch größer sein. Der Anteil der Assimilation des Fahnenblattes und der anderen grüner Organe am Aufbau der Körner ist keine statische Größe, sondern verändert sich im Laufe der Entwicklung (STOY 1973). Hinzu kommt, daß der Zusammenhang von Fläche und Leistung des Fahnenblattes stark von Prozessen der Blattalterung beeinflußt wird (APEL et al. 1973, DANTUMA 1973, GALE et al. 1974, Stamp \& HeRzog 1976). Aufgrund dieser Befunde ist es nicht möglich, Rückschlüsse von den Gaswechselmessungen an individuellen Fahnenblättern über einen Zeitabschnitt auf die $\mathrm{CO}_{2}$-Assimilation einer ganzen Pflanze oder eines ganzen Bestandes über einen Zeitabschnitt zu ziehen. Soll der Gaswechsel ganzer Bestände erfaßt werden, ist ein anderer Versuchsansatz, so wie der von BURKHART et al. (1997) nötig.

$\Rightarrow \quad$ Auf gepflügtem Boden in Reinshof zeigte die hohe mechanische Bodenbelastung über das geringe Wurzel- und Sproßwachstum bis ins dritte Versuchsjahr einen hemmenden Einfluß auf den $\mathrm{CO}_{2} / \mathrm{H}_{2} \mathrm{O}$ Gaswechsel der Fahnenblätter. Auf dem Boden mit FMW, bei dem im zweiten und dritten Versuchsjahr keine belastungsabhängigen Beeinträchtigung des Wurzelsystems auftrat, wurde auch keine Hemmung des Gaswechsels bei zunehmender Bodenbelastung gemessen.

\subsubsection{Ertragsbildung und Ertrag}

Auf den drei Standorten Reinshof, Relliehausen und Großobringen wurde im Versuchsjahr 1995 als Direktwirkung auf gepflügtem Boden ein bei steigender Belastung erheblich reduzierter Kornertrag festgestellt (Abb. 48-50). 
Die mechanische Belastung führte in Reinshof und Relliehausen zu einer Reduktion der Bestandesdichte vor dem Bestocken (Tab. 26). Dies steht in Einklang mit den Befunden von ARVIDSSON \& HÅKANSSON (1996). Die Autoren stellten auf verdichteten Böden - vor allem bei hohem Tongehalt - eine Beeinträchtigung der Saatbettqualität und eine damit verbundene Hemmung des Feldaufganges fest. Im Gegensatz dazu wurde in Großobringen - bei besonders hohem Tongehalt (Tab. 7) - keine Beeinträchtigung der Bestandesdichte festgestellt (Tab. 33). Möglicherweise ist dies auf die im Vergleich zu Reinshof und Relliehausen deutlich intensivere Saatbettbereitung mit mehrmaligem Grubber- und Kreiseleggeneinsatz zurückzuführen (Tab. 57-59, Anhang).

Im ersten Versuchsjahr wurde auf gepflügtem Boden eine positive Beziehung zwischen Wurzelsystem und Sproßwachstum festgestellt: Stark eingeschränkte Wurzelsysteme fielen mit reduziertem Sproßwachstum (Abb. 61 ) und geringem Kornertrag zusammen (Abb. 63). Die beträchtliche Einschränkung des durchwurzelten Bodens in Verbindung mit Funktionsstörungen der Wurzel könnten nicht nur Ursachen für eine beeinträchtigte Wasser- und Nährstoffaufnahme und damit für ein reduziertes Wurzel- und Sproßwachstum gewesen sein (vgl. Kap. 5.2.2), sondern auch die Ertragsbildung beeinflußt haben.

Auf allen drei Standorten wurde 1995 auf gepflügtem Boden eine bei steigender Belastung deutlich reduzierte Ährendichte und eine sinkende Einzelkornmasse ermittelt (Tab. 26, 30, 33). Wassermangel zur Zeit der Bestockung ist als Ursache möglich: Nach GEISLER (1984) ist die Anlage „produktiver“ Halme in der Phase der Bestockung besonders empfindlich gegen eine Wasserunterversorgung. Versorgen die Wurzel den Sproß in dieser Phase nicht ausreichend mit Wasser, kann die Anzahl der Halme pro Pflanze und so die Ährendichte reduziert werden. Besonders deutlich zeigte sich auf dem Standort Reinshof eine Abnahme der Einzelkornmasse bei steigender Bodenbelastung (Tab. 26). GEISLER (1984) wies darauf hin, das Wassermangel während des Wachstums der Karyopse die Einzelkornmasse mindern und zu Kümmerkorn führen kann. 


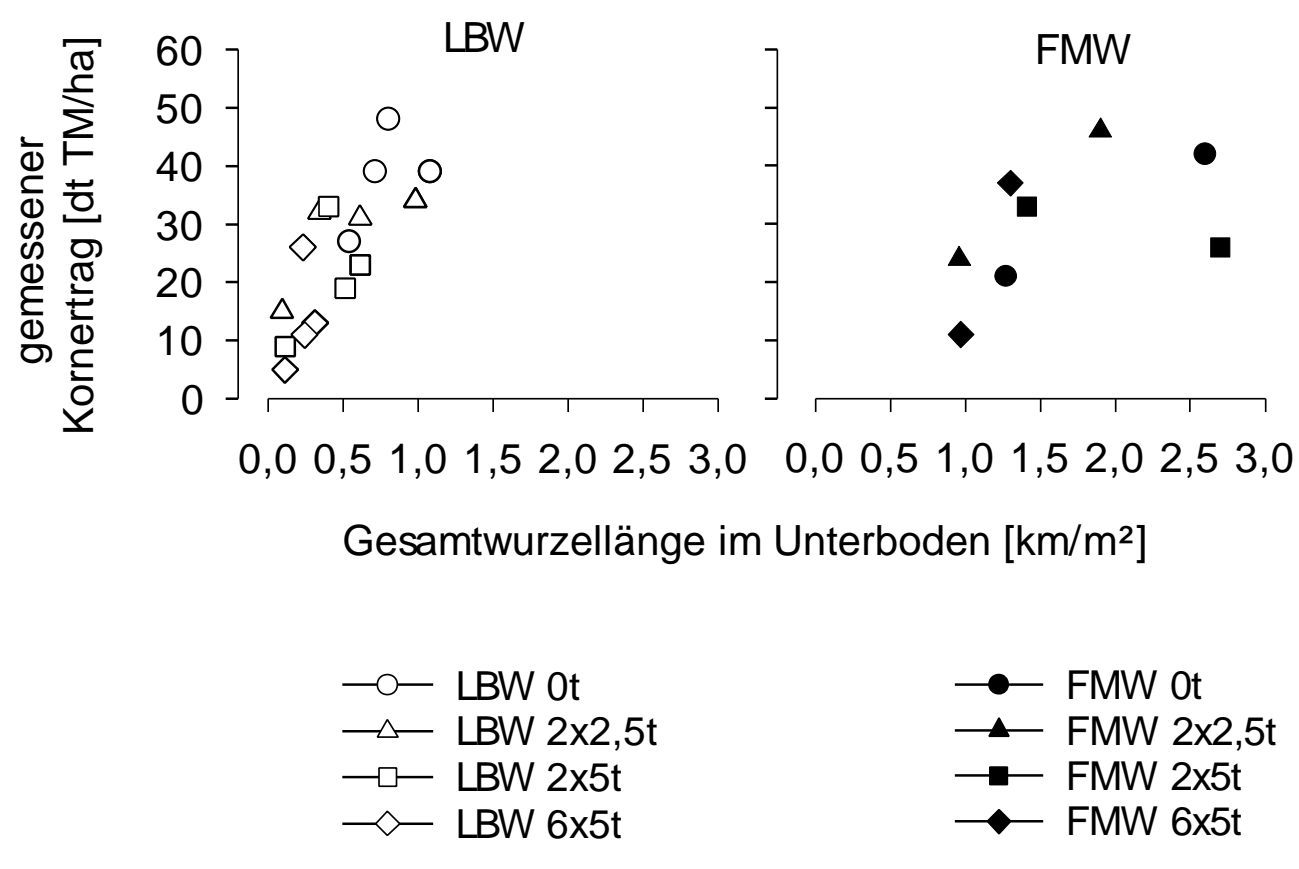

Abb. 63: Gemessener Kornertrag der Sommergerste 1995 als Funktion der Gesamtwurzellänge im Unterboden (Reinshof 26.07.95, Relliehausen 08.08.95, Großobringen 08.08.95) auf den Standorten Reinshof, Relliehausen und Großobringen. Die unterschiedlichen Bearbeitungstiefen in Großobringen (tief und flach) werden unter LBW zusammengefaßt.

Besonders drastisch zeigte sich der Einfluß der Wasserversorgung auf die Einzelkornmasse bei einem Vergleich der Standorte Reinshof und Relliehausen. Wie aus Tab. 37 hervorgeht, lag der Bodenwassergehalt des Standortes Relliehausen im Juli 1995 deutlich höher als in Reinshof. Möglicherweise führte das eingeschränkte Wasserangebot in Reinshof zu der im Vergleich mit Relliehausen um durchschnittlich 7,6 g geringeren Einzelkornmasse. Das deutlich niedrigere Gewicht der Körner in Reinshof ist wahrscheinlich auch als Ursache für den im Vergleich zu Relliehausen geringen Kornertrag anzusehen: Auf beiden Standorten lag die Ährendichte annähernd auf einer Höhe (Tab. 26, 30). Trotzdem brachte die Sommergerste in Relliehausen einen deutlich höheren Kornertrag (Abb. 48, 49). 
Tab. 37: Bodenwassergehalt auf den Standorten Reinshof (20.07.1995) und Relliehausen (05.07.1995).

\begin{tabular}{l|llll}
$\begin{array}{l}\text { Boden- } \\
\text { tiefe }[\mathrm{cm}]\end{array}$ & \multicolumn{4}{|c}{ Bodenwassergehalt [Gew. \%] } \\
\hline \multirow{3}{*}{$0-30$} & \multicolumn{3}{|c}{ Reinshof } & Relliehausen \\
& LBW & FMW & LBW & FMW \\
\cline { 2 - 4 } $30-100$ & 13,0 & 12,8 & 20,0 & 17,6 \\
& 17,8 & 16,7 & 20,0 & 19,1
\end{tabular}

In Reinshof zeigte sich bei LBW ein Zusammenhang von Bodenbelastung, Blattflächenindex und Kornertrag (Abb. 64). So wie der Kornertrag, so lag der Blattflächenindex bei geringer, mittlerer und hoher Belastung (Abb. 64) deutlich unter der Kontrolle (LBW 0t). Vermutlich war bei reduzierter Blattfläche die Bildung von Assimilaten eingeschränkt, was die Anlage der Blüten und damit die Anzahl Körner pro Ähre beeinträchtigte.

Möglicherweise wurde die Ertragsbildung auch durch einen veränderten Hormonhaushalt bei behindertem Pflanzenwachstum geprägt (vgl. Kap. 5.2.2).

Bei FMW zeigte die Direktwirkung in Reinshof nur bei hoher Belastung einen reduzierenden Einfluß auf den Kornertrag (Abb. 48). Dies bestätigte die Beobachtungen, die bei diesem Bearbeitungssystem schon bei der Auswertung der Belastungswirkung auf den Gashaushalt (Abb. 10 unten), das Wurzelsystem (Abb. 19 unten links) und das Sproßwachstum (Abb. 23) gemacht wurden: Im Vergleich zu LBW wies der Boden mit FMW eine geringere Empfindlichkeit gegenüber mechanischer Belastung auf. 

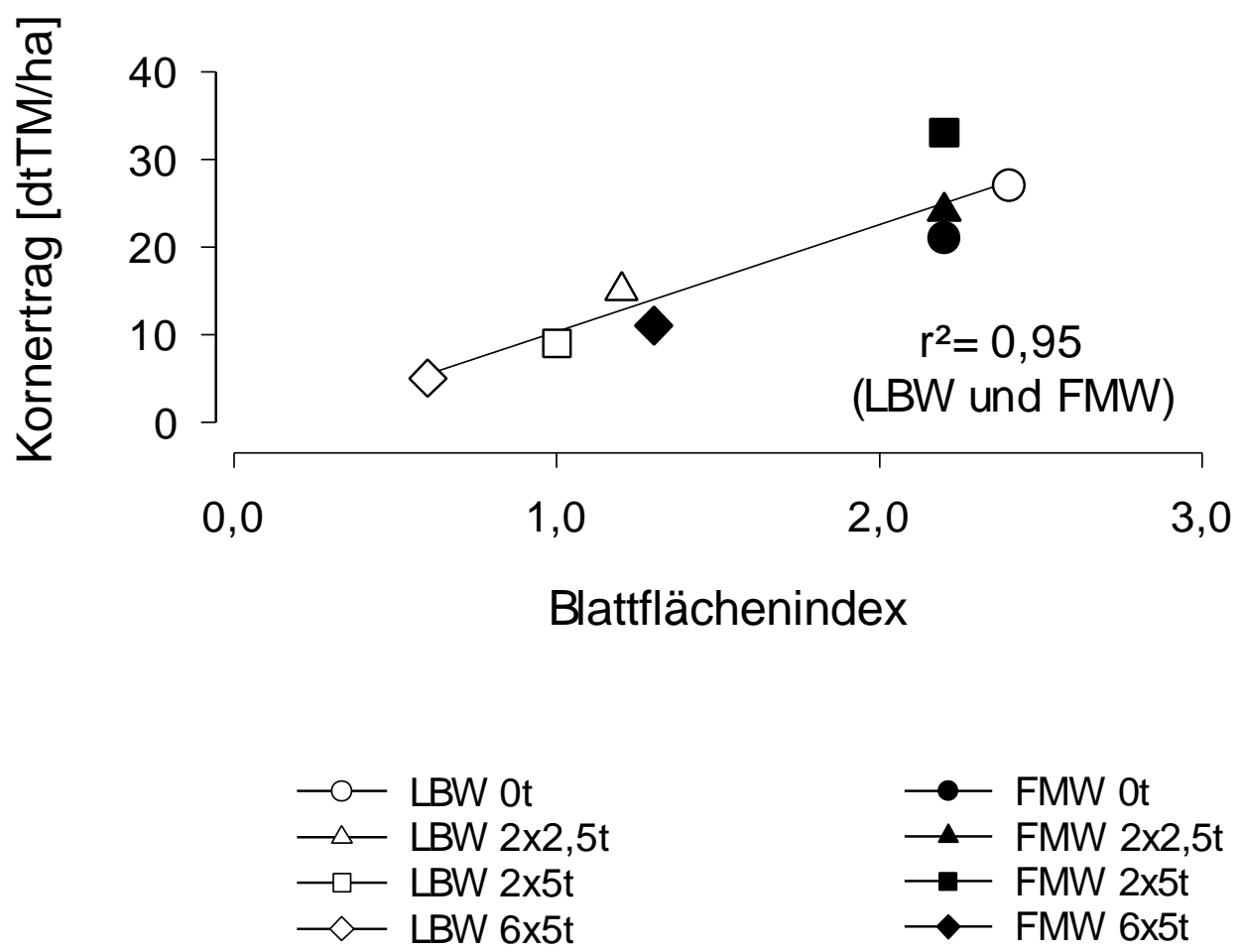

Abb. 64: Beziehung zwischen dem Kornertrag und dem mittleren Blattflächenindex von EC 25-37 der Sommergerste auf dem Standort Reinshof im Versuchsjahr 1995.

Auf Grund der hohe Toleranz des Winterweizens gegenüber verdichteten Bodenschichten mit hohem EDW und eingeschränktem Gashaushalt wurde in Reinshof und Großobringen 1996 kaum eine Beeinträchtigung der Wurzelsysteme bei steigender Belastung gemessen. Ebenso verhielt es sich mit dem gemessenen Kornertrag auf allen drei Standorten.

Aus der Ährendichte, dem Kornbesatz der Ähre und der Einzelkornmasse läßt sich ein theoretischer Kornertrag berechnen. Dieser berechnete Kornertrag spiegelt die Ertragsbildung anhand der ertragsbestimmenden Faktoren wider. Abb. 65 zeigt einen Vergleich des gemessenen Kornertrags mit dem berechneten Kornertrag des Winterweizens und der Wintergerste der Jahre 1996 und 1997 auf allen drei Standorten. Die teils große Differenz zwischen gemessenem und berechnetem Kornertrag wird auf die Heterogenität der Versuchsfelder zurückgeführt. 


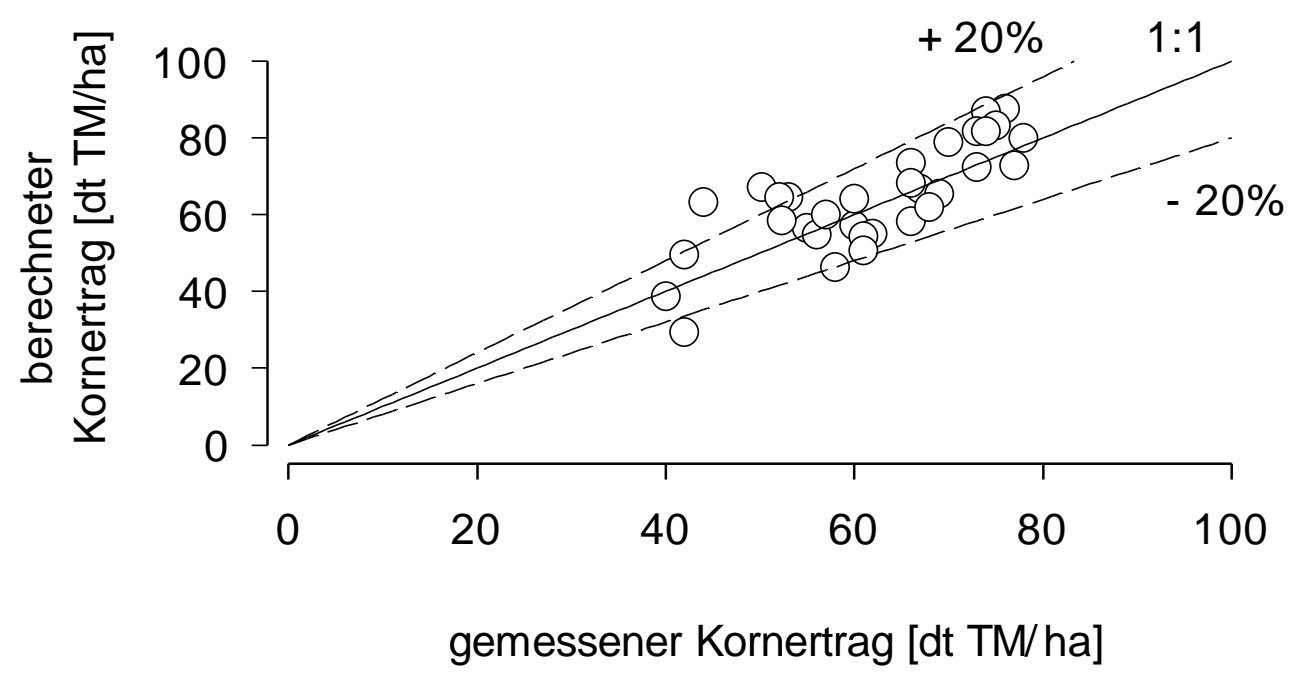

Abb. 65: Beziehung zwischen gemessenem und berechnetem Kornertrag auf den Standorten Reinshof, Relliehausen und Großobringen in den Jahren 1996 und 1997.

Im Versuchsjahr 1997 wurde auf den Standorten Relliehausen und Großobringen auf gepflügtem Boden ein tendenzieller Abfall des Kornertrags bei hoher Bodenbelastung festgestellt (Abb. 49, 50). In Reinshof wurde diese Tendenz nicht festgestellt. Wohl aber kam es in Reinshof auf gepflügtem Boden mit hoher Belastung bei stark reduziertem Wurzelsystem zu einer deutlichen Reduktion der Ährendichte. Kornbesatz und Einzelkornmasse lagen jedoch auf der Höhe der Kontrolle (LBW 0t).

Die gemessenen Kornerträge des Jahres 1997 zeigen, daß die Wintergerste auch bei stark reduziertem Wurzelsystem - wie bei LBW 2x5t und 6x5t in Reinshof (Abb. 19 rechts oben) - einen Kornertrag auf der Höhe der Kontrolle (LBW 0t) ausgebildet hat (Abb. 48). Das beweist, daß die Wintergerste in der Lage war, mögliche Beeinträchtigungen in der Versorgung mit Wasser durch das reduzierte Wurzelsystem bei der Ertragsbildung auszugleichen. Möglicherweise stand im Oberboden aber auch in ausreichendem Maße Wasser zur Verfügung. Es ist anzunehmen, daß Ertragseinbußen bei stark beeinträchtigten Wurzelsystemen mit steigender Austrocknung des Oberbodens zunehmen. Vor diesem Hintergrund gewinnen die unterschiedlichen Durchwurzelungstiefen bei LBW und FMW an Bedeutung. In Reinshof lag die maximale Durchwurzelungstiefe der Wintergerste auf dem Boden mit mittlerer Belastung bei FMW um $7 \%$ und bei hoher Belastung um 115\% höher als bei LBW (Abb. 19 rechts unten). 
$\Rightarrow$ Die belastungsabhängige Erhöhung des mechanischen Widerstandes und die drastische Einschränkung des Gashaushaltes in der Krumenbasis der Böden mit LBW hatte im ersten Versuchsjahr 1995 auf allen Standorten eine drastische Reduktion des Wurzel- und Sproßwachstums, sowie des Kornertrages zur Folge. Im Vergleich dazu trat bei FMW ein reduziertes Pflanzenwachstum und ein deutlich geringerer Kornertrag nur bei hoher Belastung auf. Im Versuchsjahr 1996 war in Reinshof eine belastungsabhängige Beeinträchtigung des Kornertrags in beiden Bearbeitungssystemen angedeutet. Im letzten Versuchsjahr (1997) zeigte sich diese Tendenz kaum. In Relliehausen wurde auf gepflügtem Boden, in Großobringen auf beiden Bearbeitungssystemen durch die Belastung der Kornertrag 1996 und 1997 in der Tendenz gemindert.

\subsection{Konsequenzen für die pflanzenbauliche Praxis}

Das Gesetz zum Schutz des Bodens fordert die Einhaltung einer guten fachlichen Praxis bei der landwirtschaftlichen Bodennutzung. Zu den im Gesetzestext angegebenen Grundsätzen einer solchen Praxis zählen unter anderem die zwei folgenden Punkte: Die Bodenbearbeitung soll unter Berücksichtigung der Witterung standortangepaßt sein. Weiterhin sollen Bodenverdichtungen so weit wie möglich vermieden werden. Insbesondere sind hierbei die Bodenart, die Bodenfeuchte und die zur Bearbeitung eingesetzten Geräte zu berücksichtigen (GesetZ ZUM SchUtZ des Bodens DER BundesRePUBliK DEUTSCHLAND 1998).

Die Ergebnisse haben gezeigt, daß die Böden der Standorte Reinshof und Relliehausen mit FMW im Vergleich zu LBW deutlich unempfindlicher gegenüber mechanischer Belastung waren: Bei annähernd gleicher Bodenfeuchte trat bei FMW eine erhebliche Beeinträchtigung der Funktion des Boden, verbunden mit einer Einschränkung des Pflanzenwachstums erst bei deutlich höherer mechanischer Belastung auf als bei LBW. Vor allem die - bei FMW fehlende pflugtypische Verdichtung der Krumenbasis stellte sich nach mittlerer und hoher Belastung bei LBW als beträchtliche Durchwurzelungsbarriere dar. Eine Regeneration des belasteten Bodens durch Rißbildung und Ausbildung von Bioporen 
trat bei FMW wesentlich schneller auf als bei LBW. Mit dem Ziel einer guten fachlichen Praxis, wie sie das Bodenschutzgesetz fordert, ist die FMW auf geeigneten Böden (EHLERS \& CLAUPEIN 1994, EHLERS 1996) in den Katalog der Maßnahmen aufzunehmen, die es dem Landwirt ermöglichen, seine Bodenbewirtschaftung standortangepaßt und bodenschonend zu gestalten.

Eine Empfehlung von Grenzwerten für eine höchstzulässige Bodenbelastung ist aus der vorliegenden Arbeit heraus sicher nicht sinnvoll. Dennoch lassen sich aus den Ergebnissen Hinweise auf Belastungsgrenzen ableiten: Bei mittlerer und hoher Belastung kam es in Reinshof auf gepflügten Böden zu drastischen Einschränkungen der Funktion des Bodens und des Wurzelwachstums bis ins dritte Versuchsjahr. Dies zeigt, schon die zweifache Überrollung mit 5t Radlast hat die Belastungsgrenze der Böden mit LBW überschritten. Durch die sechsfache Überrollung mit der gleichen Radlast wurde die Funktion des Bodens erheblich beeinträchtigt. Radlast und Überrollhäufigkeit sind zu begrenzen, um Bodenbelastungen im Sinne der Vorsorge zu vermeiden. 


\section{Zusammenfassung}

Ständig schwerer werdende Landmaschinen wie Mähdrescher, selbstfahrende Köpfrodebunker, Güllefässer und Gerätekombinationen haben zu einer erheblichen Belastung vornehmlich gepflügter Ackerböden geführt. Bei hohem Wassergehalt können vor allem gepflügte Böden (L_ockerbodenwirtschaft, LBW) belastungsempfindlich reagieren. Praktische Erfahrungen lassen die These zu, daß sich Böden mit einer flach lockernden Festboden-Mulchwirtschaft (FMW) im Vergleich zu gepflügten Böden gegenüber mechanischer Belastung als unempfindlicher erweisen und sich möglicherweise durch ein höheres Regenerationsvermögen auszeichnen. Vor diesem Hintergrund wurde in Südniedersachsen auf dem Standort Reinshof eine kolluvial überprägte Parabraunerde aus Löß und auf dem Standort Relliehausen eine Pseudogley-Parabraunerde aus Löß über Buntsandstein mit LBW und FMW nahe Feldkapazität im Frühjahr 1995 einer geringen, mittleren oder hohen mechanischen Belastung ausgesetzt. Als Kontrolle diente unbelasteter Boden mit LBW und FMW. Auf dem Standort Großobringen am Rande des Thüringer Beckens wurde tief und flach gepflügte Schwarzerde über Keuperton nach demselben Muster belastet. Auf diesem Standort wurde die Grundbodenbearbeitung mit dem Spatenpflug durchgeführt. Nach der einmaligen Bodenbelastung und der Aussaat von Sommergerste im ersten Versuchsjahr verliefen die pflanzenbaulichen Maßnahmen auf den drei Standorten betriebsüblich. Die Beobachtung der Auswirkungen der mechanischen Belastung wurde in den Jahren 1996 (Winterweizen) und 1997 (Wintergerste) fortgesetzt. Untersuchungen der bodenphysikalischen Eigenschaften, der Wurzelsysteme, des Sproßwachstums, des Gasaustausches der Pflanzen und des Kornertrags orientierten sich an der Wirkkette: mechanische Belastung $\rightarrow$ Bodeneigenschaften $\rightarrow$ Wurzelwachstum $\rightarrow$ Sproßwachstum $\rightarrow$ Ertrag.

Auf allen drei Standorten zeigte die Direktwirkung im ersten Versuchsjahr 1995 bei LBW einen Anstieg der Lagerungsdichte verbunden mit einer erheblichen Reduktion des Anteils grober Poren in der Krumenbasis als Folge der Belastung. Dies führte zu einer drastischen Erhöhung des mechanischen Widerstandes und zu einem Absinken des Gasdiffusionskoeffizienten des Bodens bei steigender Belastung. Sowohl die Untersuchung der Wurzelsysteme als auch ein Vergleich des Sproßwachstums und des Kornertrags der Sommergerste zeigten den Effekt 
der Belastung auf die bodenphysikalischen Eigenschaften der gepflügten Böden: Mit steigender Belastung wurde das Pflanzenwachstum auf allen drei Standorten erheblich eingeschränkt. Im Durchschnitt der Standorte nahm der Kornertrag der Sommergeste bei LBW im ersten Versuchsjahr von der Kontrolle bis zur hohen Belastung um $66 \%$ ab. Der pflugtypischen Krumenbasisverdichtung auf den Standorten Reinshof und Relliehausen kam besondere Bedeutung zu: Mit zunehmender Belastung stellte sich diese Bodenschicht als beträchtliche Durchwurzelungsbarriere dar, die bei geringer, mittlerer und hoher Belastung zu Ende der Vegetationszeit eine Reduktion der Gesamtwurzellänge im Unterboden um annähernd $80 \%$ bewirkte.

Im Vergleich zu LBW trat bei FMW eine erhebliche Veränderung der bodenphysikalischen Eigenschaften, verbunden mit einer beträchtlichen Reduktion des Pflanzenwachstums und des Kornertrags erst bei deutlich höherer Belastung auf. Im Durchschnitt der Standorte Reinshof und Relliehausen lag der Kornertrag 1995 bei FMW mit hoher Belastung 30\% unter dem Kornertrag auf dem Boden ohne Belastung.

In Großobringen zeigten der tief und der flach bearbeitete Boden mit LBW annähernd die gleiche Direktwirkung der Belastung wie die Böden mit LBW der Standorte Reinshof und Relliehausen. Die flache Bodenwendung mit dem Spatenpflug führte in Großobringen nicht zu einer deutlich geringeren Empfindlichkeit des Bodens gegenüber mechanischer Belastung.

Im zweiten Versuchsjahr 1996 wurden kaum belastungsabhängige Unterschiede des Pflanzenwachstums und des Kornertrags auf den drei Standorten gemessen. Der Winterweizen stellte sich gegenüber der Nachwirkung der Belastung als relativ unempfindlich dar.

Im dritten Versuchsjahr 1997 deutete in Reinshof auf gepflügtem Boden ein geringfügiger Anstieg der Gasdiffusionskoeffizienten bei geringer, mittlerer und hoher Belastung eine leichte Regeneration des belasteten Bodens an. Dennoch zeigte die Wintergerste kurz vor dem Ährenschieben bei mittlerer und hoher Belastung eine Reduktion der Gesamtwurzellänge im Unterboden von durchschnittlich 65 \%. Die Nachwirkung der hohen mechanischen Belastung bewirkte 1997 
bei LBW im Vergleich zu FMW einen Verlust von 80\% des durchwurzelten Bodens. Das Wurzelsystem der Wintergerste stellte sich im Vergleich zum Winterweizen als empfindlicher gegenüber der mechanischen Bodenbelastung dar. So wie die Wurzelsysteme, so zeigte die Bestandeshöhe der Wintergerste in Reinshof bei LBW eine Beziehung zur Belastung: Mit zunehmender Bodenbelastung war die Wuchshöhe der Pflanzen reduziert. Messungen des Gesamtwasserpotentials und des Gaswechsels am Fahnenblatt der Wintergerste in Reinshof 1997 zeigten auf gepflügtem Boden - im Gegensatz zum gemulchten, nicht gepflügten Boden - bei hoher Belastung eine geringere $\mathrm{CO}_{2}$ - Assimilation als ohne Belastung. Dies war verbunden mit einem höheren Wasserstreß. Diese Befunde legen die Vermutung nahe, daß in Reinshof bei LBW die Nachwirkung der mechanischen Belastung bis in das dritte Versuchsjahr reichte und entsprechend der Wirkkette über veränderte Bodeneigenschaften Wurzelsystem und Sproßwachstum negativ beeinflußte.

Bei FMW lagen 1997 die Diffusionskoeffizienten des belasteten Bodens auf dem Niveau des unbelasteten Bodens. Belastungsabhängige Beeinträchtigungen des Wurzelsystems, der Bestandeshöhe oder des Gaswechsels wurden nicht gemessen.

In Reinshof wurde 1997 der Kornertrag der Wintergerste bei LBW und FMW durch die Belastung nicht verändert. Im Gegensatz dazu wurde auf den Standorten Relliehausen und Großobringen bei LBW in der Tendenz ein Abfall des Kornertrags bei hoher Belastung festgestellt.

Insgesamt zeigen die Untersuchungen, daß sich die Böden mit FMW im Vergleich zu LBW durch eine höhere Unempfindlichkeit gegen mechanische Belastung und ein höheres Regenerationsvermögen auszeichnen. Die Befunde deuten darauf hin, daß FMW auf geeigneten Böden dazu beitragen kann, Nebenwirkungen der Bodenbelastung zu verringern. Die Direktwirkung der hohen Belastungsstufe hat sowohl bei LBW als auch bei FMW zu einer drastischen Beeinträchtigung von Bodenfunktionen und des Pflanzenwachstums geführt. Dies deutet darauf hin, daß bei LBW aber auch bei FMW eine Begrenzung von Radlast und Überrollhäufigkeit beachtet werden muß, wenn Bodenschutz Teil der Landbewirtschaftung sein soll. 


\section{$7 \quad$ Literatur}

Aguirrezabal L. A. N., Pellerin S. \& Tardieu F. (1993): Carbon nutritition, root branching and elongation: can the present stage of knowledge allow a predictive approach at the whole-plant level? Environmental and Experimental Botany 33, 121-130.

ALAKUKKU L. \& ELONEN P. (1994): Finnish experiments on subsoil compaction by vehicles with high axle load. Soil \& Tillage Research 29, 151-155.

Alblas J., Wanink F., van den AkKeR J. \& van der Werf H. M. G. (1994): Impact of traffic-induced compaction of sandy soils on the yield of sillage mais in The Netherlands. Soil \& Tillage Research 29, 157-165.

Apel P., Lehmann C. O. \& Friedrich O. (1973): Beziehung zwischen Fahnenblattfläche, Photosyntheserate und Einzelährenertrag bei Sommerweizen. Kulturpflanze 21, 89-95.

ARVIDSSON J. \& HÅKANSSON I. (1996): Do effects of soil compaction persist after ploughing? Results from 21 long-term field experiments in Sweden. Soil \& Tillage research 39, 175-197.

Assaeed A. M., McGowan M., Hebblethwaite P. D. \& Brereton J. C. (1990): Effect of soil compaction on growth, yield and light interception of selected crops. Annals of Applied Biology 117, 653-666.

ATWELL B. J. (1990): The effect of soil compaction on wheat during early tillering. 1. Growth, development and root structure. New Phytologist 115, 29-35.

BAEHR A.L. (1987): Selective transport of hydrocarbons in the unsaturated zone due to aqueous vapor phase partitioning. Water Resources Research 23, 1926-1938.

BAEUMER K. (1992): Allgemeiner Pflanzenbau. Ulmer, Stuttgart.

BAeUmeR K. \& PAPE G. (1972): Ergebnisse und Aussichten des Anbaus von Zuckerrüben im Ackerbausystem ohne Bodenbearbeitung. Zucker 25, 711-718.

BAKKER J.W. \& HIDDING A.P. (1970): The influence of soil structure and air content on gas diffusion in soils. Netherlands Journal of Agricultural Science 18, 37-48.

BALL B. C. (1981): Pore characteristics of soils from two cultivation experiments as shown by gas diffusivities and permeabilities and air-filled porosities. Journal of Soil Science 32, 483-498.

BARLEY K. P. (1962): The effect of localized pressure on the growth of roots. Journal of Experimental Botany 13, 95-110. 
BARRAClough P. B. \& WeIR A. H. (1988): Effects of a compacted subsoil layer on root and shoot growth, water use and nutrient uptake of winter wheat. Journal of Agricultural Science 110, 207-216.

BAUMGARTL T. (1991): Spannungsverteilung in unterschiedlich texturierten Böden und ihre Bedeutung für die Bodenstabilität. Schriftenreihe Institut für Pflanzenernährung und Bodenkunde Universität Kiel 12.

BeAdLE C. L. (1993): Growth analysis. In: Photosynthesis and Production in a Changing Environment. (Chapman \& Hall, Hrsg.), Chapman \& Hall, London.

BergmanNN W. (1954): Wurzelwachstum und Ertrag. Zeitschrift für Acker- und Pflanzenbau 97, 336-362.

Bertrand A.R. \& KohnKe H. (1957): Subsoil conditiones and their effects on oxygen supply and growths of corn roots. Soil Science Society of America Journal 21, 135-140.

BethenOd O., TARDiEU F. \& KATERJI N. (1996): Relationship between net photosynthetic rate and stomatal conductance in leaves of field-grown maize subjected to soil compaction or soil drying. Photosynthetica 32, 367379.

BlackWell P. S., Green T. W. \& Mason W. K. (1990): Response of biopore channels from roots to compression by vehicle stresses. Soil Science Society of America Journal 54, 1088-1091.

BORNSCHEUER H. (1984): Die Bodengesellschaft auf dem Systemversuch Reinshof. Diplomarbeit, Fachbereich Agrarwissenschaften, Universität Göttingen.

BöHM, W. (1979): Methods of Studying Root Systems. Ecological studies 33. Springer, Berlin.

BRANDHUBER R. (1997): Mechanische Bodenbelastung durch die landwirtschaftliche Pflanzenproduktion in Bayern. Schriftenreihe der Bayerische Landesanstalt für Bodenkultur und Pflanzenbau 7, 14-19.

BuRKHART S., MANDERSCHEID R. \& WEIGEL H. J. (1997): Bestandesgaswechselmessungen an Sommerweizen unter erhöhten atmosphärischen $\mathrm{CO}_{2}-$ Konzentrationen und Trockenstress. Mitteilungen der Gesellschaft für Pflanzenbauwissenschaften 10, 93-94.

Campell R. D. \& Moreau R. A. (1979): Ethylen in compacted soils and its effect on growth, tuber quality and yield of potatoes. American Potato Journal 56, 199-210.

CARMI A. \& HEUER B. (1981): The role of roots in control of bean shoot growth. Annual Botany 48, 519-527. 
COCKROFT B., BARLEY K.P. \& GREACEN E.L. (1969): The penetration of clays by fine probes and root tips. Australian Journal of Soil Research 7, 333348.

Colemann J. S. \& SCHNeIDER K. M. (1996): The evidence that abscisic acid does not regulate a centralized whole-plant response to low soilresource availability. Oecologia 106, 277-283.

COOTE D.R. \& RAMSEY J.F. (1983): Qualification of the effects of over 35 years of intensive cultivation on four soils. Canadian Journal of Soil Science 63, 1-14.

CURRIE J. A. (1960): Gaseous diffusion porous media. 2. Dry granular materials. British Journal of Applied Physics 11, 318-323.

Czeratzkı W. (1972): Die Ansprüche der Pflanzen an den physikalischen Bodenzustand. Landbauforscheung Völkerode 22, 29-36.

DAHL J. (1997): Das ökologische Mißverständnis und einiges andere zur Landwirtschaft. In: Landwirtschaft 97. Der kritische Agrarbericht (Agrarbündnis e.V. Bonn, Hrsg.), ABL Bauernblattverlags-GmbH, 30-33.

DANNOWSKI M. (1995): Die Auswirkungen konventioneller Landbewirtschaftung auf die nachhaltige Fruchtbarkeit des Bodens in einem Agrarlandschaftselement des Nordostdeutschen Tieflandes. Archiv für Acker- und Pflanzenbau und Bodenkunde 39, 237-259.

DANTUMA G. (1973): Rates of photosynthesis in leaves of wheat and barley varieties. Netherlands Journal of Agricultural Science 21, 181-187.

DAVIDSON R. L. (1969): Effect of root/leaf temperature differentials on root/shoot ratios in some pasture grasses and clover. Annals of Botany 33, 561569.

DAVIES W. J. \& ZHANG J. (1991): Root signals and the regulation of growth and development of plants in drying soil. Annual Review of Plant Physiology and Plant Molecular Biology 42, 55-76.

DEWIT C.T. (1991): On the efficiency of resource use in agriculture. In: Ziele und Wege der Forschung im Pflanzenbau. Festschrift für Kort Bäumer zum 65. Geburtstag (Böhm W., Hrsg.), Triade Verlag E. Claupein Göttingen, 29-54.

DEXTER A. R. (1986): Model experiments on the behaviour of roots at the interface between a tilled seed-bed and a compacted subsoil. 3. Entry of pea and wheat roots into cylindrical biopores. Plant and Soil 95, 149161.

DEXTER A. R. (1988): Advances in characterization of soil structure. Soil \& Tillage Research 11, 199-238. 
DIETZ T. (1991): Einfluß reduzierter Bodenbearbeitung auf Bodengefüge, Wasserhaushalt und Ertrag. Feldwirtschaft 32, 347-349.

DOMZAL H., GLINSKI J. \& LIPIEC J. (1991): Soil compaction research in Poland. Soil \& Tillage Research 19, 99-109.

DöRFFLING K. (1981): Das Hormonsystem der Pflanzen. Georg Thieme, Stuttgart 96-98.

DürR H. D., Petelkau H. \& SOMmeR C. (1995): Literaturstudie „Bodenverdichtung“. UBA-Texte 55, 203.

EHLERS W. (1977): Measurement and calculation of hydraulic conductivity in horizons of tilled and untilled loess-derived soil. Geoderma 19, 293-306.

EHLERS W. (1983): Auswirkungen der Bodenbelastung mit schwerem Gerät und der Bodenbearbeitung auf das Bodengefüge und das Pflanzenwachstum. KALI-Briefe (Büntehof) 16, 499-516.

EHLERS W. (1991): Wirkung von Bearbeitungssystemen auf gefügeabhängige Eigenschaften verschiedener Böden. Berichte über Landwirtschaft 204 (Sonderheft), 118-137.

EHLERS W. (1996): Wasser in Boden und Pflanze. Ulmer, Stuttgart.

EHLERS W. (1997): Optimizing the components of soil water balance by reduced and no-tillage. In: Experience with Applicability of No-tillage Crop Production in West-European Countries, Proceedings of the ECWorkshop-III- Evora, (Tebrügge F. \& Böhrnsen A., Hrsg.), Wissenschaftlicher Fachverlag Gießen 107-118.

Ehlers W., Khosla B.K., Köpke U., Stülpnagel R., Böhm W. \& Baeumer K. (1980/81): Tillage effects on root development, water uptake and growth of oats. Soil \& Tillage Research 1, 19-34.

EHLERS W., KÖPKE U., Hesse F. \& BÖHM W. (1983): Penetration resistance and root growth of oats in tilled und untilled loess soil. Soil \& Tillage Research 3, 261-275.

EHLERS W., Goss M. J. \& Boone F. R. (1986): Einfluß der Bodenbearbeitung auf Bodenwassergehalt, Durchwurzelung und Wasserentzug. KALIBriefe (Büntehof) 18, 107-125.

EHLERS W. \& TEIWES K. (1987): Der Einfluß der Bodenbearbeitung auf Porenraumgliederung, ungesättigte Wasserleitfähigkeit und Gasdiffusion: 1. Porenraumgliederung und Wasserleitung. Mitteilungen der Deutschen Bodenkundlichen Gesellschaft 53, 381-386.

EHLERS W. \& CLAUPEIN W. (1994): Approaches towards conservation tillage in Germany. In: Conservation Tillage in Temperate Agroecosystems (Carter M.R., Hrsg.), Lewis Publishers, Chelsea, Michigan (USA), 141165. 
Ehlers W., WeRner D. \& FenNer S. (1994): Reconsolidation of a deeploosened silt loam under plowing and conservation tillage. Proc. of the $13^{\text {th }}$ International Conference, ISTRO Denmark, Volume 1, 55-60.

ELLIS F. B. \& BARNES B. T. (1980): Growth and development of root systems of winter cereals grown after different tillage methods including direct drilling. Plant and Soil 55, 283-295.

ESTLER M. (1996): Praktische Bodenbearbeitung. Grundlagen, Gerätetechnik, Verfahren, Bewertung. DLG-Verlags- GmbH, Frankfurt, 108-109.

ETANA A. \& HÅKANSSON I. (1994): Swedish experiments on the persistence of subsoil compaction caused by vehicles with high axle load. Soil \& Tillage Research 29, 167-172.

FENNER S. (1995): Wirkung und Nachhaltigkeit mechanischer Lockerung von Krumenbasisverdichtungen unter Wendepflug- und Mulchwirtschaft. Dissertation, Fachbereich Agrarwissenschaften, Universität Göttingen.

Gale M. D., EdRich J. \& LuPton F. G. H. (1974): Photosynthetic rates and the effect of applied gibberellin in some dwarf, semi-dwarf and tall wheat varieties. Journal of Agricultural Science 83, 43-46.

Gameda S., Raghavan G. S. V., McKyes E., Watson A. K. \& Mehuys G. (1994): Long term effects of a single incidence of high axle load compaction on a clay soil in Quebec. Soil \& Tillage Research 29, 173-177.

GEISLER G. (1983): Ertragsphysiologie von Kulturarten des gemäßigten Klimas. Paul Parey, Hamburg.

GEISLER G. \& RITZ J. (1981): Untersuchungen zur Sproß-Wurzel-Beziehung bei Weizen. 1. Eingriff in das Wurzelwachstum und deren Bedeutung für das vegetative Wachstum der Weizenpflanze. Zeitschrift für Acker- und Pflanzenbau 150, 161-172.

GESETZ ZUM SCHUTZ DES BODENS (1998): Bundesgesetzblatt Jahrgang 1998, Teil 1, Nr. 16, ausgegeben zu Bonn am 24.03.1998. Vierter Teil: Landwirtschaftliche Bodennutzung, § 17: Gute fachliche Praxis in der Landwirtschaft.

Goss M. J. (1977): Effects of mechanical impedance on root growth in barley (Hordeum vulgare L.). Journal of Experimental Botany 28, 96-111.

Goss M. J., EHLERs W., BOONE F. R., White I. \& HOWSE K. R. (1984): Effects of soil management practice on soil physical conditions affecting root growth. Journal of Agricultural Engeneer Research 30, 131-140.

Goss M. J., HowSE K. R. \& HARRIS W. (1988): Effects of cultivation on soil water retention and use by cereals in clay soils. Journal of Soil Science $\mathbf{2 9}$, 475-488. 
Grable A. R. \& Siemer E. G. (1968): Effects of bulk density, aggregate size, and soil water suction on oxygen diffusion, redox potentials, and elongation of corn roots. Soil Science Society of America Journal 32, 180186.

GREGORY P. J. (1994): Root growth and activity. In: Physiology and Determination of Crop Yield. (Boote K. J., Bennet J. M., Sinclair T. R. \& Paulsen G. M., Hrsg.), American Society of Agronomy, Inc., Madison, Wisconsin (USA), 65-93.

GRUBER W. (1994): Der Einfluß der Bodenbearbeitung auf die Gefügestabilität von Ackerböden. In: Beurteilung von Bodenbearbeitungssystemen hinsichtlich ihrer Arbeitseffekte und deren langfristige Auswirkung auf den Boden (Tebrügge F. \& Dreier M., Hrsg.), Wissenschaftlicher Fachverlag Dr. Fleck, Gießen, 17-24.

HÅkansson I., Voorhees W. B., Elonen P., Raghavan G. S. V., LoWery B., Van WIJK A. L. M., RASMUSSEN K. \& RILEY H. (1987): Effect of high axle-load traffic on subsoil compaction and crop yield in humid regions with anual freezing. Soil and Tillage Research 10, 259-268.

HAMMEL J. E. (1994): Effect of high axle-load traffic on subsoil physical properties and crop yields in the Pacific Northwest, USA. Soil \& Tillage Research 29, 195-203.

HARTGE K.H. \& Bohne H. (1983): Der Einfluß der Gefügegeometrie auf Verdichtbarkeit des Bodens und auf Keimung von Roggen. Zeitung für Kulturtechnik und Flurbereinigung 24, 5-10.

HARTGE K. H. \& HORN R. (1991): Einführung in die Bodenphysik. Enke, Stuttgart.

HILL R.L. \& MEZA-MONTALVO M. (1990): Long-term wheel traffic effects on soil physical properties under different tillage systems. Soil Science Society of America Journal 54, 865-870.

HILLEL D. (1980): Fundamentals of Soil Physics. Academic Press, New York.

HoffmanN C. (1993): Einfluß der Bodenverdichtung auf Wurzel- und Sproßwachstum sowie die Phosphatversorgung von Zuckerrübe und Getreide. Dissertation, Fachbereich Agrarwissenschaften, Universität Göttingen.

HofFMANN C. \& JUNGK A. (1995): Growth and phosphorus supply of sugar beet as effected by soil compaction and water tension. Plant and Soil 176, 15-25.

HORN R. (1986): Auswirkungen unterschiedlicher Bodenbearbeitung auf die mechanische Belastbarkeit von Ackerböden. Zeitschrift für Pflanzenernährung und Bodenkunde 149, 9-18. 
HORN R. (1998): mündliche Mitteilungen.

HORN R., KÜHNER S. \& WiERMANN C. (1997): Was leisten Bodenbewirtschaftungsverfahren in Lehmböden für die Bodenstabilität? Landbauforschung Völkenrode, Sonderheft 178, 43-52.

HSIAO T. C. (1993): Growth and productivity of crops in relation to water status. International symposium on irrigation of horticultural crops. Almeria, Spain, 23.-27. Novermber 1992. Acta Horticultae 335, 137-148.

Johnson J. F., VoORheEs W. B., Nelson W. W. \& Randall G. W. (1990): Soybean growth and yield as affected by surface and subsoil compaction. Agronomy Journal 82, 973-979.

KAYS S. J., NiCKLOW C. W. \& SimONS D. H. (1974): Ethylen in relation to the respons of roots to physical impedence. Plant and Soil 40, 565-571.

KÖPKE U. (1979): Ein Vergleich von Feldmethoden zur Bestimmung des Wurzelwachstums landwirtschaftlicher Kulturpflanzen. Dissertation, Fachbereich Agrarwissenschaften, Universität Göttingen.

LACHNO D. R, HARRISON-MuRRAY R. S. \& Audus L. J. (1982): The effect of mechanical impedance to growth of the levels of ABA and IAA in the root tips of Zea mays (L). Journal of Experimental Botany 136, 943-951.

LANGMAACK M. (1997): Mechanische Streßverträglichkeit von Bearbeitungssystemen mit reduziertem mechanischen Eingriff. Teil 4: Beitrag von Bodentieren zur mechanischen Streßverträglichkeit. Interner Arbeitsbericht, (nicht veröffentlicht).

LANGMAAK M. (1998): Wechselwirkungen zwischen Bodenverdichtung und der Bodenfauna landwirtschaftlich genutzter Böden. Dissertation, Gemeinsame Naturwissenschaftlich Fakultät, Technische Universität, Braunschweig.

LARCHER W. (1984): Ökologie der Pflanzen. Ulmer, Stuttgart.

LEHFELDT J. (1988): Effects of plough pan compaction on root penetration of various crops grown on sandy and loamy soils. Archiv für Acker- und Pflanzenbau und Bodenkunde 32, 533-539.

LOTHSKY L., Beran P., PARIS P. \& VAligurska L. (1991): Degradation of soil by increasing compression. Soil \& Tillage Research 19, 287-295.

LOWERY B. \& SCHULER R. T. (1991): Temporal effects of subsoil compaction on soil strength and plant growth. Soil Science Society of America Journal 55, 216-223.

MARTINO D. L. \& SHAYKEWICH C. F. (1994): Root penetration profiles of wheat and barley as affected by soil penetration resistence in field conditions. Canadian Journal of Soil Science 74, 193-200. 
MASLE J. \& PASSIOURA J. B. (1987): The effect of soil strength on the growth of young wheat plants. Australian Journal of Plant Physiology 14, 643-656.

MASLE J., FARQUHAR G. D \& GIFFORD R. M. (1990): Growth and carbon economy of wheat seedlings as effected by soil resistance to penetration and ambient partial pressure of $\mathrm{CO}_{2}$. Australian Journal of Plant Physiology 17, 465-487.

Materechera S. A., Dexter A. R. \& Alston A. M. (1991): Penetration of very strong soil by seedling roots of different plant species. Plant and Soil 135, 31-41.

Materechera S. A., Alston A. M., Kirby J. M. \& Dexter A. R. (1992): Influence of root diameter on penetration of seminal roots into a compacted subsoil. Plant and Soil 144, 297-303.

McAFEE M., LINDSTROM J. \& JOHANNSSON W. (1989): Effects of presowing compaction on soil physical properties, soil atmosphere and growth of oats on a clay soil. Journal of Soil Science $40,707-717$.

MERTEN K. (1998): Das Wurzelbild von Winterweizen in einem verdichteten Lößboden bei unterschiedlicher Bodenbelastung. Diplomarbeit, Fachbereich Agrarwissenschaften, Universität Göttingen.

MOHR H. \& SCHOPFER P. (1985): Pflanzenphysiologie. Springer, Berlin.

Mullholand B. J., Black C. R., taylor I. B., Roberts J. A. \& Lenton J. R. (1996): Effect of soil compaction on barley (Hordeum vulgare L.). Possible role of ABA as a root sourced chemical signal. Journal of Experimental Botany 47, 539-549.

MunNS R. \& CRAmeR G. R. (1996): Is coordination of leaf and root growth mediated by abscisic acid? Opinion. Plant and Soil 185, 33-49.

Oussible M., CROOKSTON R. K. \& LARSON W.E. (1992): Subsurface compaction reduces the root and shoot growth and grain yield of wheat. Agronomy Journal 84, 34-38.

PASSIOURA J. B. (1994): The yield of crops in relation to drought. In: Physiology and Determination of Crop Yield. (Boote K. J., Bennet J. M., Sinclair T. R. \& Paulsen G. M., Hrsg.), American Society of Agronomy, Inc., Madison, Wisconsin (USA), 344-359.

PETELKAU H. \& DANNOWSKI M. (1990): Effect of repeated vehicle traffic lanes on soil physical properties, nutrient uptake and yield of oats. Soil \& Tillage Research 15, 217-225.

Pietola L. (1991): Effect of clay soil strength and structure on root penetration and crop yield. Annales Agriculturae Fenniae 30, 345-358. 
RAVICHANDRAN V. \& MUNGSE H. B. (1995): Effects of moisture stress on leaf area development, dry matter production and grain yield in wheat. Annals of Plant Physiology 9, 117-120.

RILEY H. (1994): The effect of traffic at high axle load on crop yields on a loam soil in Norway. Soil \& Tillage Research 29, 211-214.

RUHM E. (1983): Gute Voraussetzungen für eine schlechte Ernte. Hannoversche Land- und Forstwirtschaftliche Zeitung 136, 3-4.

RuSANOV V. A. (1991): Effects of wheel and track traffic on the soil and on the crop growth anf yield. Soil \& Tillage Research 19, 131-143.

Russel R. S. \& Goss M. J. (1974): Physical aspects of soil fertility - The response of roots to mechanical impedance. Netherlands Journal of Agricultural Science 22, 305-318.

SCHARPENBERG D. (1986): Nichtwendende Lockerung mit dem Parapflug im Vergleich zu konventioneller und reduzierter Bearbeitung: Wirkung auf biogene Durchporung des Bodens und das Wurzelwachstum von Weizen. Diplomarbeit, Institut für Pflanzenbau und Pflanzenzüchtung, Universität Göttingen.

Scheffer F. \& SchachtSchabel P. (1984): Lehrbuch der Bodenkunde. 11. Auflage, Enke, Stuttgart.

SCHJØNNING P. \& RASMUSSEN K.J. (1994): Danish experiments on subsoil compaction by vehicles with high axle load. Soil \& Tillage Research 29, 215227.

Scholander P. F., Hammel H. T., Bradstreet E. D. \& Hemmingsen E. A. (1965): Sap pressure in vascular plants. Science 148, 339-346.

Schlichting E. \& Blume H.P. (1966): Bodenkundliches Praktikum. Paul Parey, Hamburg.

SCHUURMAN J. J. (1965): Influence of soil density on root development and growth of oats. Plant and Soil 22, 352-374.

SOMMER C., DÜRR H. J. \& ZACH M. (1995): Auswirkungen von Bodenverdichtungen auf den Pflanzenertrag und Schlußfolgerungen aus der Sicht des Bodenschutzes. KTBL-Schrift 362, 176-188.

Sommer C., Ruhm E. \& AltemülleR H.J. (1981): Direkt- und Nachwirkungen starker Verdichtungen auf das Bodengefüge und den Pflanzenertrag. KALI-Briefe (Büntehof) 15, 429-448.

StAMP P \& HeRZOG H. (1976): Untersuchungen zur Fahnenblattalterung und zum Kornwachstum einiger deutscher Sommerweizensorten (Triticum aestivum L.). Zeitschrift für Pflanzenzüchtung 77, 330-338. 
STEWART G.A. \& VYN T.J. (1994): Influence of high axle loads and tillage systems on soil properties and grain corn yield. Soil \& Tillage Research 29, 229-235.

STOCKFISCH N. (1997): Strohabbau durch Mikroorganismen und Regenwürmer in zwei Bodenbearbeitungssystemen. Dissertation, Fachbereich Agrarwissenschaften, Universität Göttingen.

STOCKFISCH N., EHLERS W. \& EBERHARD U. (1995): Einfluß reduzierter Bodenbearbeitung auf biologische und chemische Kennwerte in verschiedenen Tiefen einer Löß-Parabraunerde. Mitteilungen der Deutschen Bodenkundlichen Gesellschaft 76, 697-700.

STOY V. (1973): Assimilatbildung und -verteilung als Komponente der Ertragsbildung beim Getreide. Angewandte Botanik 47, 17-26.

STÜLPNAGEL R. (1979): Ertragsbildung von Hafer auf bearbeiteten und unbearbeiteten Löß-Parabraunerden. Dissertation, Fachbereich Agrarwissenschaften, Universität Göttingen.

TARDIEU F. (1988): Analysis of the spatial variability of mais root density. 1. Effect of wheel compaction on the spatial arrangement of roots. Plant and Soil 107, 259-266.

TARDIEU F. (1994): Growth and functioning of roots and of root systems subject to soil compaction. Towards a system with multiple signalling? Soil and Tillage Research 30, 217-243.

TAYLOR H. M. \& RATLIFF L. F. (1969): Root elongation rates of cotton and peanuts as a function of soil strength and soil water content. Soil Science 108, 113-119.

TAYLOR H.M., ROBERSON G.M. \& PARKER J.J. JR. (1966): Soil strength - root penetration relations for medium - to coarse - textured soil materials. Soil Science 102, 18-22.

TEBRÜGGE F. \& BÖHRNSEN A. (1995): Beurteilung von Bearbeitungssystemen hinsichtlich Bodenschonung und Kostenentlastung. In: Zeitgemäße Bodenbearbeitungssysteme - verfahrenstechnisch effizient, ökologisch präzise (AG Landtechnik und Bauwesen Hessen e.V., Hrsg.), AG Landtechnik und Bauwesen Hessen e. V., Kassel, 27-36.

TEIWES K. (1988): Einfluß von Bodenbearbeitung und Fahrverkehr auf physikalische Eigenschaften schluffreicher Ackerböden. Dissertation, Fachbereich Agrarwissenschaften, Universität Göttingen.

THORNE G. N. (1965): Photosynthesis of ears and flag leaves of wheat and barley. Annual Botany 29, 317-329. 
ThORSTENSON D.C. \& POLLOCK D.W. (1989): Gas transport in unsaturated zones: Multicomponent systems and adequacy of Fick's laws. Water Resources Research 25, 477-507.

TORREY J. G. (1976): Root hormons and plant growth. Annual Review of Plant Physiology 27, 435-459.

VAN GENUCHTEN M.T. (1980): A closed-form equation for predicting the hydraulic conductivity of unsaturated soils. Soil Science Society of American Journal 44, 892-898.

VetteR H. \& SchaRAFAT S. (1964): Die Wurzelverbreitung landwirtschaftlicher Kulturpflanzen im Unterboden. Zeitschrift für Acker- und Pflanzenbau 120, 275-298.

VOLKMAR K. M. (1995): A method for characterizing the effect of root-formed pores on growth of roots in a chernozemic clay loam. Canadian Journal of Soil Science 75, 293-298.

VOORHEES W.B. (1983): Relative Effectiveness of tillage and natural forces in alleviating wheel-induced soil compaction. Soil Science Society of America Journal 47, 129-133.

Voorhees W.B., Senst C.G. \& Nelson W.W. (1978): Compaction and soil structure modification by wheel traffic in the northern corn belt. Soil Science Society of America Journal 42, 344-349.

VOORHEES W. B. \& LINDSTROM M. J. (1984): Long-term effects of tillage methods on soil tilth independent of wheel traffic compaction. Soil Science Society of America Journal 48, 152-156.

Voorhees W. B., Johnson J. F., Randall G. W. \& Nelson W. W. (1989): Crop growth and yield as effected by surface and subsoil compaction. Agronomy Journal 81, 294-303.

WEISCHET W. (1995): Einführung in die allgemeine Klimatologie. Teubner Studienbücher Geographie, Stuttgart.

WENDROTH O. (1990): Koeffizienten des Wasser- und Gastransportes zur Ableitung von Kenngrößen des Bodengefüges. Dissertation, Fachbereich Agrarwissenschaften, Universität Göttingen.

WERNER D. (1995): Mechanische Streßverträglichkeit von Bearbeitungssystemen mit reduziertem mechanischen Eingriff. Teil 2: Quantifizierung der Gefügeentwicklung unter differenzierter mechanischer Belastung durch Röntgen-,CT- und REM-Technik. Interner Arbeitsbericht, (nicht veröffentlicht).

WERNER D. (1996): mündliche Mitteilungen. 
WERNER D. (1998): The effects of mechanical stress and regenerative soil processes on the structure of conventionally and conservationally tilled loess soil - soil physical and image analytical findings. In: Soil Compaction and Compression in Relation to Sugar Beet Production. (Märländer B., Tijink F.G.J., Hoffmann C. \& Beckers R., Hrsg.), Internationales Institut für Rübenforschung, Göttingen, 21-33.

WiERMANN C. (1998): Auswirkungen differenzierter Bodenbearbeitung auf die Bodenstabilität und das Regenerationsvermögen lößbürtiger Ackerstandorte. Dissertation, Institut für Pflanzenernährung und Bodenkunde, Universität Kiel.

WiERSUM L. K. (1957): The relationship of the size and the structural rigidity of pores to their penetration by roots. Plant and Soil 9, 75-85.

Wilson A. J., Robards A. W., \& Goss M. J. (1977): Effects of mechanical impedance on root growth in barley (Hordeum vulgare L.) 2. Effects on cell development in seminal roots. Journal of Experimental Botany 28, 1216-1227.

WÖSTEN J.H.M. \& VAN GENUCHTEN M.T. (1988): Using texture and other soil properties to predict the unsaturated soil hydraulic functions. Soil Science Society of America Journal 52, 1762-1770.

XU X., Nieber J.L. \& GuptA S.C. (1992): Compaction effect on the gas diffusion coefficient in soils. Soil Science Society of America Journal 56, 17431750.

Young I. M., Montagu K., Conroy J. \& Bengough A. G. (1997): Mechanical impedance of root growth directly reduces leaf elongation rates of cereals. New Phytologist 135, 613-619.

ZADOKS J.C., CHANG T.T. \& KONZAK C.F. (1974): A decimal code for the growth stages of cereals. Weed Research 14, 415-421.

ZHANG J. \& TARDIEU F. (1996): Relative contribution of apices and mature tissues to ABA synthesis in droughted mais root systems. Plant Cell Physiology 35, 598-605.

ZIEGLER K. (1995): Rübentechnik - Der Siegeszug der 6-reihigen Köpfrodebunker. In: Die Zuckerrübenzeitung (Verband Süddeutscher Zuckerrübenanbauer e. V. Hrsg.) 6, 8-9. 
8 Anhang

Tab. 38: Technische Daten und Gewicht der verwendeten Fahrzeuge.

( - keine Angaben)

\begin{tabular}{l|cccc} 
Standort & $\begin{array}{l}\text { Reinshof } u \\
\text { Relliehausen }\end{array}$ & $\begin{array}{l}\text { Reinshof } u \\
\text { Relliehausen }\end{array}$ & Großobringen & Großobringen \\
\hline Fahrzeuge: & Radlader & Radlader & Kranwagen & Radlader \\
-Typ & O\&K L 35 & O\&K L 15 & Omega S15 & Ahlmann \\
-Gewicht [kg] & 18680 & 8700 & 19140 & 9780 \\
Reifentyp: & Michelin & Dunlop & Michelin & - \\
& 23.5 R25 & E91-2 & $1400-R 24$ & $15 \times 5 / 25$ \\
-Durchmesser [cm] & 149 & 130 & - & - \\
-Breite [cm] & 54 & 48 & - & - \\
Innendruck [bar] & & & 8,82 & - \\
-vorne & 3,75 & - & 8,82 & - \\
-hinten & 2,00 & & &
\end{tabular}

Tab. 39: Kontaktflächendruck bei den Belastungsstufen. Die Angaben sind zusammengestellt nach WIERMANN 1997. ( - = keine Angaben)

\begin{tabular}{|c|c|c|c|c|c|c|}
\hline \multirow[t]{2}{*}{ Standort } & \multicolumn{2}{|c|}{ Reinshof } & \multicolumn{2}{|c|}{ Relliehausen } & \multicolumn{2}{|c|}{ Großobringen } \\
\hline & LBW & FMW & LBW & FMW & LBW tief & LBW flach \\
\hline $\begin{array}{l}\text { Kontaktflächen- } \\
\text { druck [kPa] bei }\end{array}$ & & & & & & \\
\hline 2. Belastungsstufe & 77 & 111 & 96 & 105 & 127 & - \\
\hline Kontaktflächen- & & & & & & \\
\hline druck [kPa] bei & & & & & & \\
\hline 3. und 4. & & & & & & \\
\hline Belastungsstufe & 150 & 163 & 185 & 144 & 107 & - \\
\hline
\end{tabular}


Tab. 40: Die Entwicklungsstadien bei Getreide nach ZADOKS et al. (1974).

EC Keimung

00 Trockenes Saatkorn

01 Beginn der Wasseraufnahme

03 Abschluß der Wasseraufnahme

05 Austritt der Keimwurzel aus dem Saatkorn

$07 \quad$ Austritt der Koleoptile

09 Blatt erreicht die Spitze der Koleoptile

\section{Keimtriebentwicklung}

10 Auflaufen: Koleoptile duchstößt Erdoberfläche

11 1-Blatt-Stadium: 1. Blatt entfaltet

12 2-Blatt-Stadium: 2. Blatt entfaltet

13 3-Blatt-Stadium: 3. Blatt entfaltet

14-19 Viertes bis 9. und mehr Blätter entfaltet

\section{Bestockung}

20 Nur Haupttrieb vorhanden

21 Bestockungsbeginn: Haupttrieb und 1. Bestockungstrieb

22-25 Haupttrieb und 2 bis 5 Bestockungstriebe

$26 \quad$ Haupttrieb und 6 Bestockungstriebe

27-29 Haupttrieb und 7-9 und mehr Bestockungstriebe

\section{Schossen}

Schoßbeginn: Haupt- und Nebentriebe aufgerichtet

31 1. Knoten dicht über der Erdoberfläche wahrnehmbar

32 2. Knoten wahrnehmbar. Ein Stengel hat sich gebildet.

$37 \quad$ Fahnenblatt gerade sichtbar

39 Blattscheide ist geschwollen

Ligula des Fahnenblattes ist vollständig entwickelt

\section{Wachstum des Blütenstandes}

41 Fahnenblatt-Lamina entwickelt sich

43 Oberster Halmteil schwillt an

$47 \quad$ Fahnenblattscheide öffnet sich

49 Erste Grannen (soweit vorhanden) sichtbar 


\section{Blütenstand tritt hervor}

51 Beginn des Ähren- oder Rispenschiebens

52/53 $\quad 1 / 4$ des Blütenstandes sichtbar

54/55 1/2 des Blütenstandes sichtbar

56/57 3/4 des Blütenstandes sichtbar

58/59 Blütenstand voll sichtbar

\section{Blüte}

61 Blühbeginn: Die ersten Staubbeutel erscheinen

65 Vollblüte: Die meisten Ährchen haben reife Staubbeutel

69 Blühende: Sämtliche Ährchen haben geblüht

\section{Reife}

$71 \quad$ Kornbildung

73/74 Frühe Milc hreife

75/76 Mittlere Milchreife

77/79 Späte Milchreife

83 Frühe Teigreife

85 Weichteigreife

87 Gelbreife: Korninhalt plastisch bis fest

91 Vollreife: Korninhalt hart; Pflanze abgestorben

92 Totreife: Korninhalt vollständig hart

$93 \quad$ Karyopse lockert sich über Tag

94 Überreife: Stroh abgestorben und zusammenbrechend

95 Samenruhe

96 lebensfähige Körner zu 50\% keimfähig

97 Samen nicht mehr im Ruhestand

98 2. Ruhephase beginnt

99 2. Ruhephase beendet 
Tab. 41: Lagerungsdichte und Luftkapazität bei pF 1,8 auf dem Standort Reinshof 1995. Die Daten sind zusammengestellt nach Angaben der TLL, Jena.

\begin{tabular}{|c|c|c|c|c|c|c|}
\hline \multirow[t]{2}{*}{$\begin{array}{l}\text { Belastungs- } \\
\text { stufe }\end{array}$} & $\begin{array}{c}\text { Boden- } \\
\text { tiefe [cm] } \\
\text { LBW }\end{array}$ & $\begin{array}{l}\text { Lagerungs- } \\
\text { dichte }\left[\mathrm{g} / \mathrm{cm}^{3}\right]\end{array}$ & $\begin{array}{c}\text { Luft- } \\
\text { kapazität } \\
\text { [Vol. \%] }\end{array}$ & $\begin{array}{c}\text { Boden- } \\
\text { tiefe [cm] } \\
\text { FMW }\end{array}$ & $\begin{array}{l}\text { Lagerungs- } \\
\text { dichte }\left[\mathrm{g} / \mathrm{cm}^{3}\right]\end{array}$ & $\begin{array}{c}\text { Luft- } \\
\text { kapazität } \\
\text { [Vol. \%] }\end{array}$ \\
\hline & \multicolumn{3}{|c|}{ LBW } & \multicolumn{3}{|c|}{ FMW } \\
\hline $0 t$ & $\begin{array}{c}7-13 \\
18-24 \\
30-36 \\
40-46\end{array}$ & $\begin{array}{l}1,39 \\
1,51 \\
1,48 \\
1,44\end{array}$ & $\begin{array}{l}16,3 \\
10,2 \\
12,2 \\
15,7\end{array}$ & $\begin{array}{c}7-13 \\
18-24 \\
30-36 \\
40-46\end{array}$ & $\begin{array}{l}1,46 \\
1,51 \\
1,45 \\
1,43\end{array}$ & $\begin{array}{l}14,0 \\
12,5 \\
15,7 \\
17,4\end{array}$ \\
\hline $2 \times 2,5 t$ & $\begin{array}{c}7-13 \\
18-24 \\
30-36 \\
40-46\end{array}$ & $\begin{array}{l}1,46 \\
1,48 \\
1,52 \\
1,46\end{array}$ & $\begin{array}{l}15,1 \\
10,5 \\
10,4 \\
14,5\end{array}$ & $\begin{array}{c}7-13 \\
18-24 \\
30-36 \\
40-46\end{array}$ & $\begin{array}{l}1,52 \\
1,57 \\
1,43 \\
1,42\end{array}$ & $\begin{array}{c}12,4 \\
9,0 \\
16,4 \\
17,4\end{array}$ \\
\hline $2 \times 5 t$ & $\begin{array}{c}7-13 \\
18-24 \\
30-36 \\
40-46\end{array}$ & $\begin{array}{l}1,50 \\
1,49 \\
1,50 \\
1,48\end{array}$ & $\begin{array}{l}12,3 \\
10,0 \\
10,4 \\
12,2\end{array}$ & $\begin{array}{c}7-13 \\
18-24 \\
30-36 \\
40-46\end{array}$ & $\begin{array}{l}1,53 \\
1,53 \\
1,48 \\
1,46\end{array}$ & $\begin{array}{l}11,1 \\
10,9 \\
14,3 \\
15,9\end{array}$ \\
\hline $6 \times 5 t$ & $\begin{array}{c}7-13 \\
18-24 \\
30-36 \\
40-46\end{array}$ & $\begin{array}{l}1,57 \\
1,55 \\
1,52 \\
1,52\end{array}$ & $\begin{array}{r}7,5 \\
9,5 \\
9,9 \\
10,9\end{array}$ & $\begin{array}{c}7-13 \\
18-24 \\
30-36 \\
40-46\end{array}$ & $\begin{array}{l}1,60 \\
1,60 \\
1,56 \\
1,50\end{array}$ & $\begin{array}{r}7,7 \\
7,6 \\
9,5 \\
12,5\end{array}$ \\
\hline
\end{tabular}


Tab. 42: Lagerungsdichte und Luftkapazität bei pF 1,8 auf dem Standort Reinshof 1996. Die Daten sind zusammengestellt nach Angaben der TLL, Jena.

\begin{tabular}{|c|c|c|c|c|c|c|}
\hline $\begin{array}{l}\text { Belastungs- } \\
\text { stufe }\end{array}$ & $\begin{array}{c}\text { Boden- } \\
\text { tiefe [cm] } \\
\text { LBW }\end{array}$ & $\begin{array}{l}\text { Lagerungs- } \\
\text { dichte }\left[\mathrm{g} / \mathrm{cm}^{3}\right]\end{array}$ & $\begin{array}{c}\text { Luft- } \\
\text { kapazität } \\
\text { [Vol. \%] }\end{array}$ & $\begin{array}{c}\text { Boden- } \\
\text { tiefe [cm] } \\
\text { FMW }\end{array}$ & $\begin{array}{l}\text { Lagerungs- } \\
\text { dichte }\left[\mathbf{g} / \mathbf{c m}^{3}\right]\end{array}$ & $\begin{array}{c}\text { Luft- } \\
\text { kapazität } \\
\text { [Vol. \%] }\end{array}$ \\
\hline & \multicolumn{3}{|c|}{ LBW } & \multicolumn{3}{|c|}{ FMW } \\
\hline $0 t$ & $\begin{array}{c}6-12 \\
16-22 \\
30-36 \\
40-46\end{array}$ & $\begin{array}{l}1,43 \\
1,49 \\
1,53 \\
1,45\end{array}$ & $\begin{array}{l}16,7 \\
12,1 \\
11,4 \\
15,9\end{array}$ & $\begin{array}{c}4-10 \\
16-22 \\
29-35 \\
40-46\end{array}$ & $\begin{array}{l}1,44 \\
1,51 \\
1,52 \\
1,47\end{array}$ & $\begin{array}{l}13,8 \\
11,5 \\
12,1 \\
15,5\end{array}$ \\
\hline $2 \times 2,5 t$ & $\begin{array}{c}6-12 \\
16-22 \\
30-36 \\
40-46\end{array}$ & $\begin{array}{l}1,50 \\
1,49 \\
1,54 \\
1,50\end{array}$ & $\begin{array}{l}13,5 \\
13,2 \\
11,9 \\
13,5\end{array}$ & $\begin{array}{c}4-10 \\
16-22 \\
29-35 \\
40-46\end{array}$ & $\begin{array}{l}1,53 \\
1,54 \\
1,50 \\
1,49\end{array}$ & $\begin{array}{c}9,3 \\
9,5 \\
14,0 \\
13,9\end{array}$ \\
\hline $2 \times 5 t$ & $\begin{array}{c}6-12 \\
15-21 \\
30-36 \\
40-46\end{array}$ & $\begin{array}{l}1,41 \\
1,43 \\
1,58 \\
1,46\end{array}$ & $\begin{array}{c}18,6 \\
15,4 \\
9,5 \\
14,4\end{array}$ & $\begin{array}{c}4-10 \\
16-22 \\
29-35 \\
40-46\end{array}$ & $\begin{array}{l}1,51 \\
1,59 \\
1,54 \\
1,54\end{array}$ & $\begin{array}{c}9,8 \\
7,9 \\
10,9 \\
11,4\end{array}$ \\
\hline $6 \times 5 t$ & $\begin{array}{c}5-11 \\
13-19 \\
30-36 \\
40-46\end{array}$ & $\begin{array}{l}1,51 \\
1,46 \\
1,62 \\
1,53\end{array}$ & $\begin{array}{c}13,9 \\
14,6 \\
7,9 \\
11,6\end{array}$ & $\begin{array}{c}4-10 \\
16-22 \\
29-35 \\
40-46\end{array}$ & $\begin{array}{l}1,52 \\
1,66 \\
1,62 \\
1,52\end{array}$ & $\begin{array}{c}11,5 \\
6,1 \\
7,1 \\
12,1\end{array}$ \\
\hline
\end{tabular}


Tab. 43: Lagerungsdichte und Luftkapazität bei pf 1,8 auf dem Standort Reinshof 1997. Die Daten sind zusammengestellt nach Angaben der TLL, Jena.

\begin{tabular}{|c|c|c|c|c|c|c|}
\hline \multirow[t]{2}{*}{$\begin{array}{l}\text { Belastungs- } \\
\text { stufe }\end{array}$} & $\begin{array}{c}\text { Boden- } \\
\text { tiefe }[\mathrm{cm}] \\
\text { LBW }\end{array}$ & $\begin{array}{c}\text { Lagerungs- } \\
\text { dichte }\left[\mathrm{g} / \mathrm{cm}^{3}\right]\end{array}$ & $\begin{array}{c}\text { Luft- } \\
\text { kapazität } \\
\text { [Vol. \%] }\end{array}$ & $\begin{array}{c}\text { Boden- } \\
\text { tiefe }[\mathrm{cm}] \\
\text { FMW }\end{array}$ & $\begin{array}{l}\text { Lagerungs- } \\
\text { dichte }\left[\mathrm{g} / \mathrm{cm}^{3}\right]\end{array}$ & $\begin{array}{c}\text { Luft- } \\
\text { kapazität } \\
\text { [Vol. \%] }\end{array}$ \\
\hline & \multicolumn{3}{|c|}{ LBW } & \multicolumn{3}{|c|}{ FMW } \\
\hline $0 t$ & $\begin{array}{c}6-12 \\
16-22 \\
30-36 \\
40-46\end{array}$ & $\begin{array}{l}1,41 \\
1,37 \\
1,58 \\
1,47\end{array}$ & $\begin{array}{c}13,7 \\
15,9 \\
6,9 \\
12,1\end{array}$ & $\begin{array}{c}4-10 \\
16-22 \\
30-36 \\
40-46\end{array}$ & $\begin{array}{l}1,44 \\
1,54 \\
1,44 \\
1,46\end{array}$ & $\begin{array}{c}10,6 \\
8,4 \\
13,6 \\
12,0\end{array}$ \\
\hline $2 \times 2,5 t$ & $\begin{array}{c}6-12 \\
16-22 \\
27-33 \\
40-46\end{array}$ & $\begin{array}{l}1,46 \\
1,45 \\
1,55 \\
1,48\end{array}$ & $\begin{array}{c}10,4 \\
12,7 \\
9,1 \\
11,1\end{array}$ & $\begin{array}{c}4-10 \\
18-24 \\
30-36 \\
40-46\end{array}$ & $\begin{array}{l}1,51 \\
1,55 \\
1,48 \\
1,44\end{array}$ & $\begin{array}{c}6,4 \\
7,1 \\
12,5 \\
14,6\end{array}$ \\
\hline $2 \times 5 t$ & $\begin{array}{c}6-12 \\
16-22 \\
28-34 \\
40-46\end{array}$ & $\begin{array}{l}1,44 \\
1,43 \\
1,57 \\
1,48\end{array}$ & $\begin{array}{c}12,1 \\
12,5 \\
7,2 \\
11,3\end{array}$ & $\begin{array}{c}4-10 \\
17-23 \\
29-35 \\
40-46\end{array}$ & $\begin{array}{l}1,52 \\
1,55 \\
1,51 \\
1,44\end{array}$ & $\begin{array}{c}8,4 \\
7,7 \\
10,1 \\
14,4\end{array}$ \\
\hline $6 \times 5 t$ & $\begin{array}{c}6-12 \\
16-22 \\
26-32 \\
38-44\end{array}$ & $\begin{array}{l}1,47 \\
1,46 \\
1,66 \\
1,55\end{array}$ & $\begin{array}{c}10,9 \\
13,3 \\
3,7 \\
8,0\end{array}$ & $\begin{array}{c}4-10 \\
17-23 \\
29-35 \\
40-46\end{array}$ & $\begin{array}{l}1,54 \\
1,62 \\
1,58 \\
1,51\end{array}$ & $\begin{array}{c}7,8 \\
6,1 \\
7,8 \\
11,0\end{array}$ \\
\hline
\end{tabular}


Tab. 44: Lagerungsdichte und Luftkapazität bei $\mathrm{pF}$ 1,8 auf dem Standort Relliehausen 1995. Die Daten sind zusammengestellt nach Angaben der TLL, Jena.

\begin{tabular}{|c|c|c|c|c|c|c|}
\hline \multirow[t]{2}{*}{$\begin{array}{l}\text { Belastungs- } \\
\text { stufe }\end{array}$} & $\begin{array}{l}\text { Boden- } \\
\text { tiefe [cm] } \\
\text { LBW }\end{array}$ & $\begin{array}{l}\text { Lagerungs- } \\
\text { dichte }\left[\mathrm{g} / \mathrm{cm}^{3}\right]\end{array}$ & $\begin{array}{c}\text { Luft- } \\
\text { kapazität } \\
\text { [Vol. \%] }\end{array}$ & $\begin{array}{l}\text { Boden- } \\
\text { tiefe [cm] } \\
\text { FMW }\end{array}$ & $\begin{array}{l}\text { Lagerungs- } \\
\text { dichte }\left[\mathbf{g} / \mathrm{cm}^{3}\right]\end{array}$ & $\begin{array}{c}\text { Luft- } \\
\text { kapazität } \\
\text { [Vol. \%] }\end{array}$ \\
\hline & \multicolumn{3}{|c|}{ LBW } & \multicolumn{3}{|c|}{ FMW } \\
\hline $0 t$ & $\begin{array}{l}10-16 \\
20-26 \\
30-36 \\
45-51\end{array}$ & $\begin{array}{l}1,43 \\
1,39 \\
1,57 \\
1,50\end{array}$ & $\begin{array}{c}12,6 \\
12,6 \\
5,9 \\
8,7\end{array}$ & $\begin{array}{l}10-16 \\
20-26 \\
30-36 \\
45-51\end{array}$ & $\begin{array}{l}1,54 \\
1,51 \\
1,57 \\
1,54\end{array}$ & $\begin{array}{l}9,1 \\
9,0 \\
6,9 \\
7,4\end{array}$ \\
\hline $2 \times 2,5 t$ & $\begin{array}{l}10-16 \\
20-26 \\
30-36 \\
45-51\end{array}$ & $\begin{array}{l}1,48 \\
1,44 \\
1,55 \\
1,53\end{array}$ & $\begin{array}{c}9,6 \\
10,0 \\
7,0 \\
7,3\end{array}$ & $\begin{array}{l}10-16 \\
20-26 \\
30-36 \\
45-51\end{array}$ & $\begin{array}{l}1,58 \\
1,50 \\
1,58 \\
1,51\end{array}$ & $\begin{array}{l}6,8 \\
9,7 \\
6,3 \\
7,2\end{array}$ \\
\hline $2 \times 5 t$ & $\begin{array}{l}10-16 \\
20-26 \\
30-36 \\
45-51\end{array}$ & $\begin{array}{l}1,49 \\
1,44 \\
1,66 \\
1,56\end{array}$ & $\begin{array}{l}8,7 \\
8,3 \\
4,1 \\
7,3\end{array}$ & $\begin{array}{l}10-16 \\
20-26 \\
30-36 \\
45-51\end{array}$ & $\begin{array}{l}1,60 \\
1,58 \\
1,59 \\
1,57\end{array}$ & $\begin{array}{l}4,6 \\
7,3 \\
5,4 \\
6,7\end{array}$ \\
\hline $6 \times 5 t$ & $\begin{array}{l}10-16 \\
20-26 \\
30-36 \\
45-51\end{array}$ & $\begin{array}{l}1,48 \\
1,55 \\
1,65 \\
1,58\end{array}$ & $\begin{array}{l}9,0 \\
7,3 \\
4,1 \\
7,3\end{array}$ & $\begin{array}{l}10-16 \\
20-26 \\
30-36 \\
45-51\end{array}$ & $\begin{array}{l}1,61 \\
1,58 \\
1,65 \\
1,54\end{array}$ & $\begin{array}{l}5,3 \\
6,9 \\
4,3 \\
7,8\end{array}$ \\
\hline
\end{tabular}


Tab. 45: Lagerungsdichte und Luftkapazität bei $\mathrm{pF}$ 1,8 auf dem Standort Relliehausen 1996. Die Daten sind zusammengestellt nach Angaben der TLL, Jena.

\begin{tabular}{|c|c|c|c|c|c|c|}
\hline \multirow[t]{2}{*}{$\begin{array}{l}\text { Belastungs- } \\
\text { stufe }\end{array}$} & $\begin{array}{l}\text { Boden- } \\
\text { tiefe [cm] } \\
\text { LBW }\end{array}$ & $\begin{array}{l}\text { Lagerungs- } \\
\text { dichte }\left[\mathrm{g} / \mathrm{cm}^{3}\right]\end{array}$ & $\begin{array}{c}\text { Luft- } \\
\text { kapazität } \\
\text { [Vol. \%] }\end{array}$ & $\begin{array}{c}\text { Boden- } \\
\text { tiefe [cm] } \\
\text { FMW }\end{array}$ & $\begin{array}{l}\text { Lagerungs- } \\
\text { dichte }\left[\mathbf{g} / \mathrm{cm}^{3}\right]\end{array}$ & $\begin{array}{c}\text { Luft- } \\
\text { kapazität } \\
\text { [Vol. \%] }\end{array}$ \\
\hline & \multicolumn{3}{|c|}{ LBW } & \multicolumn{3}{|c|}{ FMW } \\
\hline $0 t$ & $\begin{array}{c}4-10 \\
16-22 \\
30-36 \\
40-46\end{array}$ & $\begin{array}{l}1,30 \\
1,30 \\
1,47 \\
1,52\end{array}$ & $\begin{array}{c}20,7 \\
21,3 \\
11,8 \\
9,6\end{array}$ & $\begin{array}{c}4-10 \\
16-22 \\
30-36 \\
40-46\end{array}$ & $\begin{array}{l}1,49 \\
1,51 \\
1,59 \\
1,51\end{array}$ & $\begin{array}{c}12,5 \\
9,7 \\
11,2 \\
8,8\end{array}$ \\
\hline $2 \times 2,5 t$ & $\begin{array}{c}4-10 \\
16-22 \\
30-36 \\
40-46\end{array}$ & $\begin{array}{l}1,39 \\
1,40 \\
1,57 \\
1,54\end{array}$ & $\begin{array}{c}17,0 \\
16,2 \\
7,0 \\
7,9\end{array}$ & $\begin{array}{c}4-10 \\
16-22 \\
30-36 \\
40-46\end{array}$ & $\begin{array}{l}1,51 \\
1,52 \\
1,53 \\
1,50\end{array}$ & $\begin{array}{c}11,4 \\
9,9 \\
11,2 \\
8,8\end{array}$ \\
\hline $2 \times 5 t$ & $\begin{array}{c}4-10 \\
16-22 \\
30-36 \\
40-46\end{array}$ & $\begin{array}{l}1,37 \\
1,43 \\
1,59 \\
1,67\end{array}$ & $\begin{array}{c}18,2 \\
15,3 \\
6,2 \\
5,3\end{array}$ & $\begin{array}{c}4-10 \\
16-22 \\
30-36 \\
40-46\end{array}$ & $\begin{array}{l}1,48 \\
1,58 \\
1,61 \\
1,68\end{array}$ & $\begin{array}{c}12,0 \\
8,1 \\
7,2 \\
5,1\end{array}$ \\
\hline $6 \times 5 t$ & $\begin{array}{c}4-10 \\
16-22 \\
30-36 \\
40-46\end{array}$ & $\begin{array}{l}1,23 \\
1,29 \\
1,60 \\
1,64\end{array}$ & $\begin{array}{c}25,9 \\
22,7 \\
5,9 \\
4,7\end{array}$ & $\begin{array}{c}4-10 \\
16-22 \\
30-36 \\
40-46\end{array}$ & $\begin{array}{l}1,42 \\
1,62 \\
1,60 \\
1,57\end{array}$ & $\begin{array}{c}14,8 \\
6,7 \\
6,7 \\
6,5\end{array}$ \\
\hline
\end{tabular}


Tab. 46: Lagerungsdichte und Luftkapazität bei pF 1,8 auf dem Standort Relliehausen 1997. Die Daten sind zusammengestellt nach Angaben der TLL, Jena.

\begin{tabular}{|c|c|c|c|}
\hline $\begin{array}{l}\text { Belastungs- } \\
\text { stufe }\end{array}$ & $\begin{array}{c}\text { Boden- } \\
\text { tiefe }[\mathrm{cm}] \\
\text { LBW }\end{array}$ & $\begin{array}{c}\text { Lagerungs- } \\
\text { dichte }\left[\mathrm{g} / \mathrm{cm}^{3}\right] \\
\text { LBW }\end{array}$ & $\begin{array}{c}\text { Luft- } \\
\text { kapazität } \\
\text { [Vol. \%] }\end{array}$ \\
\hline $0 t$ & $\begin{array}{l}4-10 \\
14-20 \\
24-30 \\
40-46\end{array}$ & $\begin{array}{l}1,38 \\
1,36 \\
1,52 \\
1,45\end{array}$ & $\begin{array}{c}13,6 \\
15,1 \\
8,4 \\
14,3\end{array}$ \\
\hline $2 \times 2,5 t$ & $\begin{array}{c}4-10 \\
12-18 \\
23-29 \\
40-46\end{array}$ & $\begin{array}{l}1,42 \\
1,39 \\
1,55 \\
1,52\end{array}$ & $\begin{array}{c}12,4 \\
13,8 \\
7,2 \\
9,7\end{array}$ \\
\hline $2 \times 5 t$ & $\begin{array}{c}3-9 \\
10-16 \\
20-26 \\
40-46\end{array}$ & $\begin{array}{l}1,36 \\
1,36 \\
1,56 \\
1,53\end{array}$ & $\begin{array}{c}15,0 \\
16,0 \\
6,4 \\
10,1\end{array}$ \\
\hline $6 \times 5 t$ & $\begin{array}{c}2-8 \\
8-14 \\
18-24 \\
40-46\end{array}$ & $\begin{array}{l}1,47 \\
1,45 \\
1,58 \\
1,52\end{array}$ & $\begin{array}{c}9,9 \\
11,7 \\
5,8 \\
9,3\end{array}$ \\
\hline
\end{tabular}

\begin{tabular}{ccc}
$\begin{array}{c}\text { Boden- } \\
\text { tiefe } \mathbf{c m} \text { ] } \\
\text { FMW }\end{array}$ & $\begin{array}{c}\text { Lagerungs- } \\
\text { dichte }\left[\mathbf{g} / \mathbf{c m}^{3} \text { ] }\right.\end{array}$ & $\begin{array}{c}\text { Luft- } \\
\text { kapazität } \\
\text { [Vol. \%] }\end{array}$ \\
\hline $4-10$ & FMW & \\
$16-22$ & 1,48 & 9,9 \\
$30-36$ & 1,51 & 9,7 \\
$40-46$ & 1,56 & 7,8 \\
$4-10$ & 1,50 & 9,7 \\
$16-22$ & 1,50 & 9,9 \\
$30-36$ & 1,51 & 10,2 \\
$40-46$ & 1,57 & 8,1 \\
$4-10$ & 1,49 & 10,2 \\
$16-22$ & 1,49 & 10,0 \\
$30-36$ & 1,53 & 9,4 \\
$40-46$ & 1,63 & 5,6 \\
$4-10$ & 1,54 & 6,9 \\
$16-22$ & 1,46 & 10,3 \\
$30-36$ & 1,56 & 9,0 \\
$40-46$ & 1,60 & 5,6 \\
& 1,52 & 9,4
\end{tabular}


Tab. 47: Lagerungsdichte und Luftkapazität bei pF 1,8 auf dem Standort Großobringen 1995. Die Daten sind zusammengestellt nach Angaben der TLL, Jena.

\begin{tabular}{|c|c|c|c|}
\hline $\begin{array}{l}\text { Belastungs- } \\
\text { stufe }\end{array}$ & $\begin{array}{l}\text { Boden- } \\
\text { tiefe [cm] } \\
\text { LBW tief }\end{array}$ & $\begin{array}{c}\begin{array}{c}\text { Lagerungs- } \\
\text { dichte }\left[\mathbf{g} / \mathrm{cm}^{3}\right] \\
\text { LBW tief }\end{array} \\
\end{array}$ & $\begin{array}{c}\text { Luft- } \\
\text { kapazität } \\
\text { [Vol. \%] }\end{array}$ \\
\hline $0 t$ & $\begin{array}{l}3-11 \\
15-22 \\
28-36 \\
39-46\end{array}$ & $\begin{array}{l}1,32 \\
1,44 \\
1,45 \\
1,44\end{array}$ & $\begin{array}{l}17,0 \\
12,1 \\
12,1 \\
12,6\end{array}$ \\
\hline $2 \times 2,5 t$ & $\begin{array}{c}3-11 \\
15-22 \\
28-36 \\
39-46\end{array}$ & $\begin{array}{l}1,50 \\
1,56 \\
1,41 \\
1,43\end{array}$ & $\begin{array}{r}6,8 \\
6,9 \\
13,0 \\
13,1\end{array}$ \\
\hline $2 \times 5 t$ & $\begin{array}{l}3-11 \\
15-22 \\
28-36 \\
39-46\end{array}$ & $\begin{array}{l}1,54 \\
1,55 \\
1,53 \\
1,44\end{array}$ & $\begin{array}{r}5,3 \\
5,2 \\
7,3 \\
10,9\end{array}$ \\
\hline $6 \times 5 t$ & $\begin{array}{c}3-11 \\
15-22 \\
28-36 \\
39-46\end{array}$ & $\begin{array}{l}1,54 \\
1,60 \\
1,51 \\
1,50\end{array}$ & $\begin{array}{l}5,9 \\
4,3 \\
5,6 \\
7,3\end{array}$ \\
\hline
\end{tabular}

\begin{tabular}{ccc}
$\begin{array}{c}\text { Boden- } \\
\text { tiefe [cm] } \\
\text { LBW flach }\end{array}$ & $\begin{array}{c}\text { Lagerungs- } \\
\text { dichte }\left[\mathbf{g} / \mathbf{c m}^{3} \text { ] }\right.\end{array}$ & $\begin{array}{c}\text { Luft- } \\
\text { kapazität } \\
\text { [Vol. \%] }\end{array}$ \\
\hline $3-11$ & LBW flach & \\
\hline $15-22$ & 1,26 & 19,2 \\
$28-36$ & 1,50 & 8,6 \\
$39-46$ & 1,50 & 8,4 \\
$3-11$ & 1,47 & 9,2 \\
$15-22$ & 1,51 & 6,8 \\
$28-36$ & 1,53 & 7,2 \\
$39-46$ & 1,50 & 8,7 \\
$3-11$ & 1,46 & 10,0 \\
$15-22$ & 1,54 & 5,1 \\
$28-36$ & 1,55 & 5,1 \\
$39-46$ & 1,53 & 7,2 \\
$3-11$ & 1,45 & 10,6 \\
$15-22$ & 1,54 & 5,8 \\
$28-36$ & 1,57 & 4,4 \\
$39-46$ & 1,53 & 5,6 \\
& 1,54 & 7,5
\end{tabular}


Tab. 48: Lagerungsdichte und Luftkapazität bei pF 1,8 auf dem Standort Großobringen 1996. Die Daten sind zusammengestellt nach Angaben der TLL, Jena.

\begin{tabular}{c|ccc}
$\begin{array}{c}\text { Belastungs- } \\
\text { stufe }\end{array}$ & $\begin{array}{c}\text { Boden- } \\
\text { tiefe [cm] } \\
\text { LBW tief }\end{array}$ & $\begin{array}{c}\text { Lagerungs- } \\
\text { dichte [g/cm }]\end{array}$ & $\begin{array}{c}\text { Luft- } \\
\text { kapazität } \\
\text { [Vol. \%] }\end{array}$ \\
$\mathbf{0 t}$ & LBW tief & \\
\hline \multirow{4}{*}{$\mathbf{2 \times 2 5 t}$} & $5-11$ & 1,33 & 19,6 \\
& $16-23$ & 1,48 & 11,3 \\
& $29-35$ & 1,50 & 8,1 \\
$\mathbf{2 x 5 t}$ & $40-46$ & 1,45 & 8,4 \\
& $5-11$ & 1,32 & 20,0 \\
& $16-22$ & 1,49 & 9,9 \\
& $29-35$ & 1,47 & 12,3 \\
$\mathbf{6 x 5 t}$ & $40-46$ & 1,44 & 10,8 \\
& $3-9$ & 1,33 & 19,5 \\
& $15-21$ & 1,49 & 10,3 \\
& $27-33$ & 1,56 & 5,6 \\
& $39-45$ & 1,47 & 10,6 \\
& $4-10$ & 1,36 & 19,3 \\
& $16-22$ & 1,55 & 6,8 \\
& $27-33$ & 1,53 & 6,8 \\
& $40-46$ & 1,48 & 9,4
\end{tabular}

\begin{tabular}{ccc}
$\begin{array}{c}\text { Boden- } \\
\text { tiefe [cm] } \\
\text { LBW flach }\end{array}$ & $\begin{array}{c}\text { Lagerungs- } \\
\text { dichte [g/cm } \mathbf{~} \text { ] }\end{array}$ & $\begin{array}{c}\text { Luft- } \\
\text { kapazität } \\
\text { [Vol. \%] }\end{array}$ \\
\hline $5-11$ & LBW flach & \\
\hline $16-22$ & 1,28 & 22,0 \\
$29-35$ & 1,39 & 15,8 \\
$39-45$ & 1,40 & 11,4 \\
$5-11$ & 1,43 & 10,4 \\
$14-20$ & 1,34 & 18,6 \\
$28-34$ & 1,51 & 9,9 \\
$39-45$ & 1,47 & 8,8 \\
$3-9$ & 1,47 & 10,1 \\
$14-20$ & 1,30 & 20,6 \\
$28-34$ & 1,42 & 13,6 \\
$39-45$ & 1,59 & 5,2 \\
$3-9$ & 1,46 & 8,0 \\
$14-20$ & 1,30 & 21,6 \\
$27-34$ & 1,52 & 9,2 \\
$41-47$ & 1,57 & 4,9 \\
& 1,50 & 7,1
\end{tabular}


Tab. 49: Lagerungsdichte und Luftkapazität bei pF 1,8 auf dem Standort Großobringen 1997. Die Daten sind zusammengestellt nach Angaben der TLL, Jena.

\begin{tabular}{|c|c|c|c|}
\hline $\begin{array}{l}\text { Belastungs- } \\
\text { stufe }\end{array}$ & $\begin{array}{l}\text { Boden- } \\
\text { tiefe [cm] } \\
\text { LBW tief }\end{array}$ & $\begin{array}{c}\begin{array}{c}\text { Lagerungs- } \\
\text { dichte }\left[\mathrm{g} / \mathrm{cm}^{3}\right]\end{array} \\
\text { LBW tief }\end{array}$ & $\begin{array}{c}\text { Luft- } \\
\text { kapazität } \\
\text { [Vol. \%] }\end{array}$ \\
\hline $0 t$ & $\begin{array}{c}5-11 \\
16-22 \\
30-36 \\
40-46\end{array}$ & $\begin{array}{l}1,40 \\
1,54 \\
1,44 \\
1,41\end{array}$ & $\begin{array}{c}13,2 \\
8,1 \\
10,8 \\
11,3\end{array}$ \\
\hline $2 \times 2,5 t$ & $\begin{array}{c}5-11 \\
16-22 \\
30-36 \\
40-46\end{array}$ & $\begin{array}{l}1,40 \\
1,49 \\
1,43 \\
1,38\end{array}$ & $\begin{array}{c}12,2 \\
9,8 \\
11,1 \\
12,4\end{array}$ \\
\hline $2 \times 5 t$ & $\begin{array}{c}4-10 \\
17-23 \\
30-36 \\
40-46\end{array}$ & $\begin{array}{l}1,37 \\
1,55 \\
1,43 \\
1,39\end{array}$ & $\begin{array}{c}14,9 \\
7,4 \\
10,2 \\
12,0\end{array}$ \\
\hline $6 \times 5 t$ & $\begin{array}{c}5-11 \\
16-22 \\
30-36 \\
40-46\end{array}$ & $\begin{array}{l}1,42 \\
1,59 \\
1,55 \\
1,44\end{array}$ & $\begin{array}{c}14,3 \\
7,0 \\
5,3 \\
9,0\end{array}$ \\
\hline
\end{tabular}

\begin{tabular}{ccc}
$\begin{array}{c}\text { Boden- } \\
\text { tiefe [cm] } \\
\text { LBW flach }\end{array}$ & $\begin{array}{c}\text { Lagerungs- } \\
\text { dichte }\left[\mathbf{g} / \mathbf{c m}^{3} \text { ] }\right.\end{array}$ & $\begin{array}{c}\text { Luft- } \\
\text { kapazität } \\
\text { [Vol. \%] }\end{array}$ \\
\hline $16-22$ & LBW flach & \\
\hline $24-30$ & 1,42 & 12,0 \\
$30-36$ & 1,53 & 6,6 \\
$40-46$ & 1,46 & 7,8 \\
$14-20$ & 1,45 & 10,2 \\
$22-28$ & 1,38 & 13,9 \\
$30-36$ & 1,55 & 6,0 \\
$40-46$ & 1,42 & 10,1 \\
$12-18$ & 1,42 & 12,9 \\
$22-28$ & 1,35 & 14,3 \\
$30-36$ & 1,58 & 5,0 \\
$40-46$ & 1,49 & 6,8 \\
$12-18$ & 1,46 & 8,7 \\
$22-28$ & 1,31 & 18,9 \\
$30-36$ & 1,56 & 6,3 \\
$40-46$ & 1,53 & 5,5 \\
& 1,51 & 6,1
\end{tabular}


Tab. 50: Wurzellängendichte der Sommergerste in der Bodenmatrix (idBm) und in Bioporen (iBp) auf dem Standort Reinshof am 03.07.1995 (EC45).

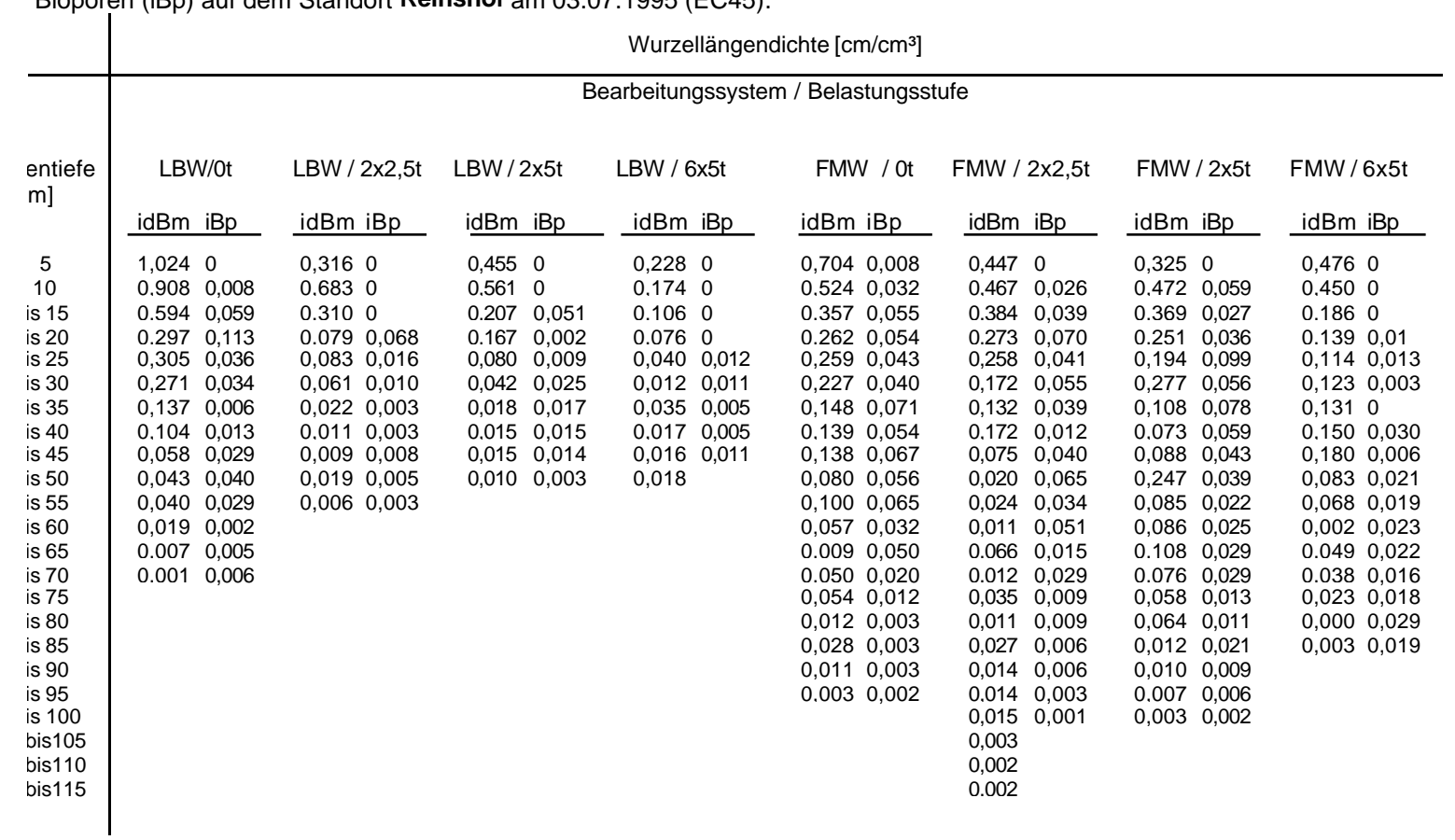


Tab. 51: Wurzellängendichte der Sommergerste in der Bodenmatrix (idBm) und in

Bioporen (iBp) auf dem Standort Reinshof am 26.07.1995 (EC 85).

Wurzellängendichte $\left[\mathrm{cm} / \mathrm{cm}^{3}\right]$

Bearbeitungssystem / Belastungsstufe

\begin{tabular}{|c|c|c|c|c|c|c|c|c|c|c|}
\hline $\begin{array}{l}\text { identiefe } \\
\text { [cm] }\end{array}$ & $\begin{array}{r}\text { LBW / Ot } \\
\mathrm{idBm} \mathrm{iBp} \\
\end{array}$ & $\begin{array}{l}\text { LBW / } 2 \times 2,5 \mathrm{t} \\
\mathrm{idBm} \mathrm{iBp} \\
\end{array}$ & $\begin{array}{l}\text { LBW / 2x5t } \\
\text { idBm iBp }\end{array}$ & $\begin{array}{l}\text { LBW / 6x5t } \\
\text { idBm iBp }\end{array}$ & $\begin{array}{l}\text { FMW / 0t } \\
\mathrm{idBm} i \mathrm{Bp}\end{array}$ & $\begin{array}{l}\text { FMW / } 2 \times 2,5 \mathrm{t} \\
\underline{\mathrm{idBm} i \mathrm{Bp}} \\
\end{array}$ & $\begin{array}{l}\text { FMW } \\
\text { idBm } \\
\end{array}$ & $/ 2 \times 5 t$ & $\mathrm{idBm}$ & $6 \times 5 t$ \\
\hline is 5 & $0,593 \quad 0$ & 0,6140 & $0,554 \quad 0$ & 0,5200 & $0,476 \quad 0$ & $0,550 \quad 0$ & 0,470 & 0,018 & 0,450 & 0 \\
\hline is 10 & $0,524 \quad 0,035$ & $0,503 \quad 0,014$ & $\begin{array}{lll}0,535 & 0,048\end{array}$ & $0,488 \quad 0,018$ & $0,568 \quad 0,005$ & $0,570 \quad 0$ & 0,725 & 0,051 & 0,766 & 0,003 \\
\hline bis 15 & $0,416 \quad 0,013$ & $0,185 \quad 0,021$ & $0,248 \quad 0,067$ & $0,131 \quad 0,007$ & 0,2720 & $0,366 \quad 0,007$ & 0,283 & 0 & 0,335 & 0 \\
\hline bis 20 & $0,296 \quad 0,067$ & $0,105 \quad 0,036$ & $0,107 \quad 0,019$ & 0,1110 & $0,210 \quad 0,015$ & $0,258 \quad 0,011$ & 0,233 & 0,005 & 0,158 & 0,002 \\
\hline bis 25 & $0,188 \quad 0,091$ & $0,108 \quad 0,02$ & $0,121 \quad 0,014$ & $0,106 \quad 0,012$ & $0,171 \quad 0,025$ & $0,145 \quad 0,051$ & 0,201 & 0,002 & 0,111 & 0 \\
\hline bis 30 & $0,196 \quad 0,02$ & $0,051 \quad 0$ & 0,0830 & $0,124 \quad 0,013$ & $0,152 \quad 0,039$ & $0,156 \quad 0,014$ & 0,159 & 0,012 & 0,156 & 0 \\
\hline bis 35 & $0,122 \quad 0,003$ & $0,011 \quad 0,026$ & 0,0310 & $0,037 \quad 0,019$ & $0,148 \quad 0,009$ & $0,137 \quad 0,051$ & 0,162 & 0,036 & 0,129 & 0,004 \\
\hline bis 40 & $0,090 \quad 0$ & $0,010 \quad 0,005$ & $0,021 \quad 0$ & $0,010 \quad 0,015$ & $0,108 \quad 0,005$ & $0,045 \quad 0,042$ & 0,153 & 0,032 & 0,103 & 0,005 \\
\hline bis 45 & $0,093 \quad 0,026$ & $0,011 \quad 0,002$ & $0,018 \quad 0,003$ & 0,011 & $0,076 \quad 0,019$ & $\begin{array}{ll}0,056 & 0,019\end{array}$ & 0,112 & 0,022 & 0,106 & 0,007 \\
\hline bis 50 & $0,111 \quad 0,003$ & $0,019 \quad 0,001$ & $0,000 \quad 0,001$ & & $0,093 \quad 0,019$ & $0,049 \quad 0,019$ & 0,088 & 0,019 & 0,066 & 0,009 \\
\hline bis 55 & $0,0690,001$ & $0,001 \quad 0,003$ & $0,0140,001$ & & $0,0030,090$ & $0,036 \quad 0,032$ & 0,060 & 0,033 & 0,082 & 0,039 \\
\hline bis 60 & $0,038 \quad 0,005$ & 0,004 & $0,001 \quad 0,007$ & & $0,0590,009$ & $0,038 \quad 0,005$ & 0,086 & 0,003 & 0,082 & 0,062 \\
\hline bis 65 & $0,023 \quad 0,005$ & 0,005 & $0,001 \quad 0,002$ & & $0,038 \quad 0,012$ & $0,018 \quad 0,003$ & 0,060 & 0,019 & 0,081 & 0,033 \\
\hline bis 70 & 0,021 & & 0,009 & & $0,038 \quad 0,009$ & $0,005 \quad 0,015$ & 0,053 & 0,005 & 0,089 & 0,046 \\
\hline bis 75 & 0,003 & & 0,002 & & $0,033 \quad 0,023$ & $0,018 \quad 0,013$ & 0,045 & 0,009 & 0,023 & 0,044 \\
\hline bis 80 & & & & & $0,013 \quad 0,012$ & $0,014 \quad 0,002$ & 0,022 & 0,009 & 0,035 & 0,009 \\
\hline bis 85 & & & & & $0,009 \quad 0,005$ & 0,017 & 0,003 & 0,008 & 0,025 & 0,012 \\
\hline bis 90 & & & & & $0,007 \quad 0,016$ & 0,010 & 0,007 & 0,003 & 0,022 & 0,023 \\
\hline bis 95 & & & & & $0,000 \quad 0,009$ & 0,009 & 0,012 & 0,003 & 0,010 & \\
\hline bis 100 & & & & & 0,006 & 0,013 & 0,002 & 0,006 & 0,005 & \\
\hline 0 bis 105 & & & & & 0,001 & & 0,001 & 0,003 & 0,002 & \\
\hline 5 bis 110 & & & & & 0,001 & & 0,002 & & 0,001 & \\
\hline
\end{tabular}


Tab. 52: Wurzellängendichte des Winterweizens auf dem Standort Reinshof am 15.05.1996 (EC 26).

\begin{tabular}{|c|c|c|c|c|c|c|c|c|}
\hline \multirow[b]{3}{*}{$\begin{array}{r}\text { 3odentiefe } \\
{[\mathrm{cm}]}\end{array}$} & \multicolumn{8}{|c|}{ Wurzellängendichte $\left[\mathrm{cm} / \mathrm{cm}^{3}\right]$} \\
\hline & \multicolumn{8}{|c|}{ Bearbeitungssystem / Belastungsstufe } \\
\hline & LBW / Ot & LBW / $2 \times 2,5 \mathrm{t}$ & LBW / $2 x 5 t$ & LBW / 6x5t & $\mathrm{FMW} / 0 \mathrm{t}$ & FMW / $2 \times 2,5 \mathrm{t}$ & FMW / $2 \times 5 t$ & $\mathrm{FMW} / 6 \times 5 \mathrm{t}$ \\
\hline bis 5 & 0,779 & 0,730 & 0,693 & 0,574 & 0,726 & 0,714 & 0,688 & 0,598 \\
\hline$i$ bis 10 & 0,739 & 0,589 & 0,509 & 0,591 & 0,664 & 0,595 & 0,627 & 0,467 \\
\hline 0 bis 15 & 0,598 & 0,485 & 0,282 & 0,321 & 0,508 & 0,336 & 0,391 & 0,211 \\
\hline 5 bis 20 & 0,441 & 0,349 & 0,162 & 0,231 & 0,383 & 0,229 & 0,177 & 0,143 \\
\hline 0 bis 25 & 0,306 & 0,301 & 0,193 & 0,154 & 0,319 & 0,208 & 0,201 & 0,123 \\
\hline 5 bis 30 & 0,135 & 0,206 & 0,060 & 0,068 & 0,223 & 0,225 & 0,193 & 0,117 \\
\hline 0 bis 35 & 0,109 & 0,088 & 0,043 & 0,060 & 0,166 & 0,145 & 0,136 & 0,106 \\
\hline 5 bis 40 & 0,078 & 0,077 & 0,032 & 0,038 & 0,081 & 0,114 & 0,120 & 0,073 \\
\hline 0 bis 45 & 0,059 & 0,026 & 0,065 & 0,073 & 0,056 & 0,080 & 0,120 & 0,043 \\
\hline 5 bis 50 & 0,033 & 0,047 & 0,049 & 0,088 & 0,025 & 0,064 & 0,099 & 0,058 \\
\hline 0 bis 55 & 0,042 & 0,018 & 0,046 & 0,059 & 0,013 & 0,033 & 0,048 & 0,018 \\
\hline 5 bis 60 & 0,029 & 0,013 & 0,030 & 0,034 & 0,010 & 0,020 & 0,029 & 0,037 \\
\hline io bis 65 & 0,005 & 0,017 & 0,009 & 0,011 & 0,016 & 0,012 & 0,040 & 0,013 \\
\hline 5 bis 70 & 0,005 & 0,021 & 0,002 & 0,005 & 0,015 & 0,008 & 0,017 & 0,000 \\
\hline 0 bis 75 & 0,014 & 0,024 & 0,004 & 0,005 & 0,010 & 0,013 & 0,005 & 0,004 \\
\hline ' 5 bis 80 & 0,001 & 0,030 & 0,002 & 0,004 & 0,018 & 0,005 & 0,004 & 0,000 \\
\hline 0 bis 85 & 0,002 & 0,002 & & 0,001 & 0,012 & 0,004 & 0,000 & 0,004 \\
\hline i5 bis 90 & 0,008 & & & & 0,005 & & 0,009 & 0,005 \\
\hline 10 bis 95 & 0,003 & & & & 0,003 & & 0,009 & \\
\hline 15 bis 100 & 0,002 & & & & 0,001 & & 0,003 & \\
\hline 00 bis 105 & 0,001 & & & & 0,001 & & 0,003 & \\
\hline 05 bis 110 & & & & & & & 0,001 & \\
\hline 10 bis115 & & & & & & & & \\
\hline
\end{tabular}


Tab. 53: Wurzellängendichte des Winterweizens auf dem Standort Reinshof am 12.06.1996 (EC 50).

Wurzellängendichte $\left[\mathrm{cm} / \mathrm{cm}^{3}\right]$

\begin{tabular}{|c|c|c|c|c|c|c|c|c|}
\hline \multirow[b]{2}{*}{$\begin{array}{c}\text { Bodentiefe } \\
\lceil\mathrm{cm}\rceil\end{array}$} & \multicolumn{8}{|c|}{ Bearbeitungssystem / Belastungsstufe } \\
\hline & $\mathrm{LBW} / \mathrm{Ot}$ & LBW / $2 \times 2,5 \mathrm{t}$ & LBW / 2x5t & LBW / $6 x 5 t$ & $\mathrm{FMW} / 0 \mathrm{t}$ & FMW / 2x2,5t & FMW / 2x5t & FMW / $6 x 5 t$ \\
\hline 0 bis 5 & 0,941 & 0,895 & 0,965 & 0,767 & 0,883 & 0,841 & 0,789 & 0,736 \\
\hline 5 bis 10 & 0,945 & 0,765 & 0,648 & 0,696 & 0,675 & 0,598 & 0,569 & 0,510 \\
\hline 10 bis 15 & 0,710 & 0,998 & 0,795 & 0,708 & 0,335 & 0,458 & 0,548 & 0,360 \\
\hline 15 bis 20 & 0,615 & 0,554 & 0,459 & 0,548 & 0,359 & 0,469 & 0,125 & 0,425 \\
\hline 20 bis 25 & 0,551 & 0,425 & 0,325 & 0,474 & 0,167 & 0,169 & 0,299 & 0,339 \\
\hline 25 bis 30 & 0,318 & 0,365 & 0,399 & 0,290 & 0,124 & 0,125 & 0,320 & 0,230 \\
\hline 30 bis 35 & 0,269 & 0,236 & 0,125 & 0,167 & 0,160 & 0,265 & 0,400 & 0,310 \\
\hline 35 bis 40 & 0,260 & 0,256 & 0,222 & 0,158 & 0,159 & 0,211 & 0,159 & 0,187 \\
\hline 40 bis 45 & 0,136 & 0,154 & 0,329 & 0,102 & 0,123 & 0,154 & 0,143 & 0,100 \\
\hline 45 bis 50 & 0,100 & 0,269 & 0,026 & 0,079 & 0,056 & 0,089 & 0,125 & 0,137 \\
\hline 50 bis 55 & 0,075 & 0,059 & 0,098 & 0,093 & 0,064 & 0,048 & 0,125 & 0,137 \\
\hline 55 bis 60 & 0,067 & 0,024 & 0,098 & 0,148 & 0,089 & 0,090 & 0,110 & 0,088 \\
\hline 60 bis 65 & 0,078 & 0,020 & 0,098 & 0,155 & 0,122 & 0,130 & 0,025 & 0,058 \\
\hline 65 bis 70 & 0,022 & 0,010 & 0,075 & 0,139 & 0,100 & 0,090 & 0,050 & 0,077 \\
\hline 70 bis 75 & 0,013 & 0,001 & 0,002 & 0,049 & 0,060 & 0,035 & 0,006 & 0,034 \\
\hline 75 bis 80 & 0,005 & 0,002 & 0,009 & 0,004 & 0,047 & 0,006 & 0,001 & 0,026 \\
\hline 80 bis 85 & 0,019 & 0,002 & 0,008 & 0,001 & 0,057 & 0,005 & 0,001 & 0,019 \\
\hline 85 bis 90 & 0,019 & 0,001 & & & 0,050 & 0,004 & 0,001 & 0,006 \\
\hline 90 bis 95 & 0,010 & 0,001 & & & 0,045 & 0,001 & 0,001 & 0,013 \\
\hline 95 bis 100 & 0,001 & 0,001 & & & 0,012 & 0,001 & 0,002 & 0,001 \\
\hline 100 bis 105 & 0,001 & 0,001 & & & 0,001 & 0,005 & 0,001 & \\
\hline 105 bis 110 & 0,001 & & & & 0,001 & & 0,001 & \\
\hline 110 bis 115 & 0,003 & & & & 0,001 & & 0,001 & \\
\hline 115 bis 120 & 0,001 & & & & 0,001 & & & \\
\hline 120 bis 125 & 0,001 & & & & & & & \\
\hline
\end{tabular}


Tab. 54: Wurzellängendichte des Winterweizens auf dem Standort Reinshof am 17.07.1996 (EC 71).

Wurzellängendichte $\left[\mathrm{cm} / \mathrm{cm}^{3}\right]$

\begin{tabular}{|c|c|c|c|c|c|c|c|c|}
\hline \multirow[b]{2}{*}{$\begin{array}{c}\text { Bodentiefe } \\
{[\mathrm{cm}]}\end{array}$} & \multicolumn{8}{|c|}{ Bearbeitungssystem / Belastungsstufe } \\
\hline & LBW / Ot & LBW / $2 \times 2,5 t$ & LBW / $2 \times 5 t$ & LBW / $6 \times 5 \mathrm{t}$ & $\mathrm{FMW} / 0 \mathrm{t}$ & $\mathrm{FMW} / 2 \times 2,5 \mathrm{t}$ & FMW / $2 \times 5 t$ & $\mathrm{FMW} / 6 \times 5 \mathrm{t}$ \\
\hline 0 bis 5 & 0,844 & 0,939 & 0,860 & 0,973 & 0,874 & 0,845 & 0,701 & 0,765 \\
\hline $\begin{array}{l}5 \text { bis } 10 \\
10 \text { bis } 15\end{array}$ & $\begin{array}{l}0,824 \\
0,783\end{array}$ & $\begin{array}{l}0,960 \\
0,588\end{array}$ & $\begin{array}{l}0,540 \\
0,780\end{array}$ & $\begin{array}{l}0,711 \\
0,609\end{array}$ & $\begin{array}{l}0,972 \\
0,685\end{array}$ & $\begin{array}{l}0,740 \\
0,690\end{array}$ & $\begin{array}{l}0,625 \\
0,623\end{array}$ & $\begin{array}{l}0,635 \\
0,387\end{array}$ \\
\hline 15 bis 20 & 0,618 & 0,712 & 0,632 & 0,593 & 0,458 & 0,359 & 0,316 & 0,318 \\
\hline 20 bis 25 & 0,590 & 0,450 & 0,490 & 0,429 & 0,433 & 0,546 & 0,322 & 0,304 \\
\hline 25 bis 30 & 0,421 & 0,365 & 0,590 & 0,380 & 0,428 & 0,455 & 0,326 & 0,215 \\
\hline 30 bis 35 & 0,210 & 0,215 & 0,149 & 0,193 & 0,338 & 0,326 & 0,336 & 0,173 \\
\hline 35 bis 40 & 0,189 & 0,189 & 0,125 & 0,121 & 0,253 & 0,265 & 0,365 & 0,130 \\
\hline 40 bis 45 & 0,188 & 0,204 & 0,125 & 0,129 & 0,274 & 0,265 & 0,256 & 0,070 \\
\hline 45 bis 50 & 0,179 & 0,165 & 0,120 & 0,126 & 0,244 & 0,265 & 0,215 & 0,069 \\
\hline 50 bis 55 & 0,136 & 0,199 & 0,129 & 0,224 & 0,188 & 0,244 & 0,158 & 0,071 \\
\hline 55 bis 60 & 0,122 & 0,099 & 0,100 & 0,130 & 0,125 & 0,265 & 0,125 & 0,056 \\
\hline 60 bis 65 & 0,098 & 0,098 & 0,045 & 0,089 & 0,083 & 0,154 & 0,089 & 0,074 \\
\hline 65 bis 70 & 0,098 & 0,048 & 0,058 & 0,085 & 0,071 & 0,099 & 0,056 & 0,064 \\
\hline 70 bis 75 & 0,043 & 0,045 & 0,056 & 0,041 & 0,081 & 0,054 & 0,046 & 0,034 \\
\hline 75 bis 80 & 0,067 & 0,015 & 0,035 & 0,015 & 0,044 & 0,025 & 0,059 & 0,051 \\
\hline 80 bis 85 & 0,040 & 0,020 & 0,025 & 0,017 & 0,047 & 0,011 & 0,045 & 0,056 \\
\hline 85 bis 90 & 0,028 & 0,026 & 0,036 & 0,011 & 0,057 & 0,009 & 0,066 & 0,037 \\
\hline 90 bis 95 & 0,029 & 0,003 & 0,006 & 0,004 & 0,028 & 0,006 & 0,003 & 0,038 \\
\hline 95 bis 100 & 0,010 & 0,002 & 0,006 & 0,001 & 0,055 & 0,006 & 0,002 & 0,023 \\
\hline 100 bis 105 & 0,001 & 0,001 & 0,004 & & 0,022 & 0,001 & 0,001 & \\
\hline 105 bis 110 & 0,001 & 0,001 & 0,001 & & 0,006 & 0,001 & 0,001 & \\
\hline 110 bis 115 & 0,004 & & & & 0,004 & & 0,001 & \\
\hline 115 bis120 & & & & & 0,001 & & & \\
\hline 120 bis 125 & & & & & 0,001 & & & \\
\hline
\end{tabular}


Tab. 55: Wurzellängendichte der Wintergerste auf dem Standort Reinshof am 14.05.1997 (EC 31).

Wurzellängendichte $\left[\mathrm{cm} / \mathrm{cm}^{3}\right]$

\begin{tabular}{|c|c|c|c|c|c|c|c|c|}
\hline \multirow[b]{2}{*}{$\begin{array}{r}\text { Bodentiefe } \\
{[\mathrm{cm}]}\end{array}$} & \multicolumn{8}{|c|}{ Bearbeitungssystem / Belastungsstufe } \\
\hline & LBW / Ot & LBW / $2 \times 2,5 \mathrm{t}$ & LBW / $2 \times 5 t$ & LBW / $6 \times 5 \mathrm{t}$ & FMW / Ot & FMW / $2 \times 2,5 t$ & FMW / $2 \times 5 t$ & $\mathrm{FMW} / 6 \times 5$ \\
\hline 0 bis 5 & 0,530 & 0,599 & 0,469 & 0,450 & 0,560 & 0,660 & 0,520 & 0,550 \\
\hline $\begin{array}{l}5 \text { bis } 10 \\
10 \text { bis } 15\end{array}$ & $\begin{array}{l}0,560 \\
0,458\end{array}$ & $\begin{array}{l}0,488 \\
0,458\end{array}$ & $\begin{array}{l}0,458 \\
0,457\end{array}$ & $\begin{array}{l}0,510 \\
0,330\end{array}$ & $\begin{array}{l}0,456 \\
0,420\end{array}$ & $\begin{array}{l}0,560 \\
0,420\end{array}$ & $\begin{array}{l}0,330 \\
0,340\end{array}$ & $\begin{array}{l}0,420 \\
0,320\end{array}$ \\
\hline 15 bis 20 & 0,250 & 0,359 & 0,266 & 0,359 & 0,260 & 0,326 & 0,330 & 0,420 \\
\hline 20 bis 25 & 0,233 & 0,220 & 0,240 & 0,240 & 0,190 & 0,366 & 0,360 & 0,210 \\
\hline 25 bis 30 & 0,132 & 0,199 & 0,145 & 0,210 & 0,200 & 0,112 & 0,110 & 0,190 \\
\hline 30 bis 35 & 0,093 & 0,084 & 0,089 & 0,065 & 0,154 & 0,011 & 0,084 & 0,145 \\
\hline 35 bis 40 & 0,069 & 0,052 & 0,064 & 0,035 & 0,086 & 0,050 & 0,075 & 0,092 \\
\hline 40 bis 45 & 0,056 & 0,054 & 0,042 & 0,026 & 0,075 & 0,044 & 0,054 & 0,065 \\
\hline 45 bis 50 & 0,034 & 0,049 & 0,010 & & 0,078 & 0,069 & 0,080 & 0,079 \\
\hline 50 bis 55 & 0,036 & 0,036 & 0,006 & & 0,054 & 0,036 & 0,056 & 0,056 \\
\hline 55 bis 60 & 0,012 & 0,026 & 0,004 & & 0,046 & 0,023 & 0,025 & 0,065 \\
\hline 60 bis 65 & 0,024 & 0,023 & 0,005 & & 0,009 & 0,006 & 0,006 & 0,009 \\
\hline 65 bis 70 & 0,021 & 0,005 & 0,002 & & 0,003 & 0,005 & 0,005 & 0,006 \\
\hline 70 bis 75 & 0,008 & 0,003 & 0,001 & & 0,003 & 0,003 & 0,002 & 0,006 \\
\hline 75 bis 80 & 0,003 & 0,003 & 0,002 & & 0,002 & 0,006 & 0,009 & 0,003 \\
\hline 80 bis 85 & 0,004 & 0,005 & 0,001 & & 0,002 & 0,002 & 0,005 & 0,006 \\
\hline 85 bis 90 & 0,002 & 0,003 & & & 0,002 & & & 0,001 \\
\hline 90 bis 95 & 0,001 & 0,001 & & & 0,005 & & & 0,002 \\
\hline 100 bis 105 & & & & & & & & \\
\hline 105 bis 110 & & & & & & & & \\
\hline 110 bis 115 & & & & & & & & \\
\hline 115 bis 120 & & & & & & & & \\
\hline
\end{tabular}


Tab. 56: Wurzellängendichte der Wintergerste auf dem Standort Reinshof am 02.06.1997 (EC 45).

Wurzellängendichte $\left[\mathrm{cm} / \mathrm{cm}^{3}\right]$

Bearbeitungssystem / Belastungsstufe

\begin{tabular}{|c|c|c|c|c|c|c|c|c|}
\hline $\begin{array}{r}\text { Bodentiefe } \\
{[\mathrm{cm}]}\end{array}$ & LBW / Ot & LBW / 2x2,5t & LBW / 2x5t & LBW / 6x5t & FMW / Ot & $\mathrm{FMW} / 2 \times 2,5 \mathrm{t}$ & FMW / 2x5t & FMW / $6 x 5 t$ \\
\hline 0 bis 5 & 0,700 & 0,776 & 0,720 & 0,710 & 0,826 & 0,833 & 0,803 & 0,677 \\
\hline 5 bis 10 & 0,802 & 0,819 & 0,416 & 0,419 & 0,553 & 0,630 & 0,371 & 0,524 \\
\hline 10 bis 15 & 0,609 & 0,505 & 0,316 & 0,391 & 0,264 & 0,440 & 0,240 & 0,357 \\
\hline 15 bis 20 & 0,389 & 0,368 & 0,239 & 0,333 & 0,177 & 0,266 & 0,194 & 0,300 \\
\hline 20 bis 25 & 0,338 & 0,313 & 0,177 & 0,240 & 0,109 & 0,191 & 0,090 & 0,160 \\
\hline 25 bis 30 & 0,161 & 0,157 & 0,069 & 0,068 & 0,082 & 0,139 & 0,069 & 0,100 \\
\hline 30 bis 35 & 0,116 & 0,098 & 0,092 & 0,090 & 0,081 & 0,111 & 0,057 & 0,104 \\
\hline 35 bis 40 & 0,075 & 0,090 & 0,098 & 0,065 & 0,053 & 0,052 & 0,059 & 0,084 \\
\hline 40 bis 45 & 0,062 & 0,059 & 0,053 & 0,003 & 0,087 & 0,028 & 0,113 & 0,102 \\
\hline 45 bis 50 & 0,081 & 0,036 & 0,021 & & 0,077 & 0,011 & 0,059 & 0,101 \\
\hline 50 bis 55 & 0,097 & 0,120 & 0,016 & & 0,086 & 0,039 & 0,036 & 0,029 \\
\hline 55 bis 60 & 0,072 & 0,050 & 0,018 & & 0,092 & 0,026 & 0,032 & 0,036 \\
\hline 60 bis 65 & 0,087 & 0,089 & 0,030 & & 0,047 & 0,030 & 0,032 & 0,054 \\
\hline 65 bis 70 & 0,008 & 0,034 & 0,013 & & 0,040 & 0,046 & 0,030 & 0,042 \\
\hline 70 bis 75 & 0,038 & 0,008 & 0,002 & & 0,020 & 0,010 & 0,029 & 0,029 \\
\hline 75 bis 80 & 0,039 & 0,011 & 0,001 & & 0,032 & 0,006 & 0,010 & 0,025 \\
\hline 80 bis 85 & 0,016 & 0,019 & & & 0,011 & 0,005 & 0,005 & 0,020 \\
\hline 85 bis 90 & 0,007 & 0,011 & & & 0,001 & & 0,011 & 0,010 \\
\hline 90 bis 95 & 0,004 & 0,017 & & & 0,004 & & 0,004 & 0,004 \\
\hline 95 bis 100 & 0,003 & 0,009 & & & 0,003 & & & 0,001 \\
\hline 100 bis 105 & 0,001 & 0,001 & & & & & & \\
\hline 105 bis 110 & & 0,001 & & & & & & \\
\hline 110 bis115 & & & & & & & & \\
\hline 115 bis 120 & & & & & & & & \\
\hline
\end{tabular}


Tab. 57: Pflanzenbauliche Maßnahmen auf dem Standort Reinshof für die Bearbeitungssysteme Lockerbodenwirtschaft (LBW) und Festboden-Mulchwirtschaft (FMW) in den Jahren 1995 bis 1997.

\begin{tabular}{|c|c|c|}
\hline $\begin{array}{l}\text { Datum } \\
12 . / 13 . \\
04.95\end{array}$ & $\begin{array}{l}\text { Maßnahme } \\
\text { Versuchsanlage }\end{array}$ & Beschreibung \\
\hline 13.04 .95 & Saatbettbereitung & Kreiselegge, Tiefe: $10 \mathrm{~cm}$ \\
\hline $\begin{array}{l}13.04 .95 \\
20.04 .95 \\
18.0595\end{array}$ & Aussaat & $\begin{array}{l}\text { Sommergerste, Sorte:Maresi } \\
\text { Feldaufgang } \\
40 \text { a }\end{array}$ \\
\hline 06.06 .95 & Herbizideinsatz & $\begin{array}{l}\text { 11/ha Starane + 11/ha Duplo DP } \\
+1,5 \mathrm{l} / \mathrm{ha} \text { IPU }\end{array}$ \\
\hline $\begin{array}{l}15.08 .95 \\
28.08 .95 \\
18.09 .95 \\
05.10 .95\end{array}$ & $\begin{array}{l}\text { Ernte } \\
\text { Stoppelbearbeitung } \\
\text { Stoppelbearbeitung } \\
\text { Grundbodenbearbeitung }\end{array}$ & $\begin{array}{l}\text { Ernteparzellen mit der Sense } \\
\text { Kreiselegge, Tiefe: } 5 \mathrm{~cm} \\
\text { Kreiselegge, Tiefe: } 5 \mathrm{~cm}, 2 \times \text { diagonal } \\
\text { LBW: Streichblechpflug, Tiefe: } 25 \mathrm{~cm} \\
\text { FMW: keine Bearbeitung }\end{array}$ \\
\hline 06.10 .95 & Saatbettbereitung & Kreiselegge, Tiefe: $5 \mathrm{~cm}$ \\
\hline 06.10 .95 & Aussaat & Winterweizen, Sorte: Pequital \\
\hline 16.10 .95 & & Feldaufgang \\
\hline 27.03 .96 & $\begin{array}{l}\text { Herbizideinsatz / } \\
+\mathrm{N} \text {-Düngung }\end{array}$ & $\begin{array}{l}1,3 \mathrm{I} / \mathrm{ha} \text { IPU + } \\
72 \mathrm{~kg} \mathrm{~N} / \mathrm{ha} \text { als Ammonnitrat- } \\
\text { Harnstofflösung }\end{array}$ \\
\hline 08.05 .96 & $\begin{array}{l}\text { N-Düngung/ } \\
+ \text { Herbizideinsatz/ } \\
\text { + Halmverkürzer }\end{array}$ & $\begin{array}{l}20 \mathrm{~kg} \mathrm{~N} / \mathrm{ha} \text { als Ammonnitrat- } \\
\text { Harnstofflösung }+0,6 \mathrm{l} / \mathrm{ha} \text { Starane }+ \\
1,0 \mathrm{l} / \mathrm{ha} \text { Optica NP }+0,8 \mathrm{l} / \mathrm{ha} \\
\text { Chlorcholinchlorid }\end{array}$ \\
\hline 14.06 .96 & Fungizideinsatz & 1,3 I/ha Opus Top \\
\hline 17.06 .96 & N-Düngung & $60 \mathrm{~kg} \mathrm{~N} / \mathrm{ha}$ als Kalkammonsalperter \\
\hline 23.08 .96 & Ernte & Ernteparzellen mit der Sense \\
\hline 04.09 .96 & Stoppelbearbeitung & Kreiselegge, Tiefe: $5 \mathrm{~cm}, 2 \times$ diagonal \\
\hline 23.09 .96 & Grundbodenbearbeitung & $\begin{array}{l}\text { LBW: Streichblechpflug, Tiefe: } 25 \mathrm{~cm} \\
\text { FMW: keine Bearbeitung }\end{array}$ \\
\hline $\begin{array}{l}23.09 .96 \\
23.09 .96 \\
03.10 .96\end{array}$ & $\begin{array}{l}\text { Stoppelbearbeitung } \\
\text { Aussaat }\end{array}$ & $\begin{array}{l}\text { Kreiselegge, Tiefe: } 5 \mathrm{~cm} \\
\text { Wintergerste, Sorte: Theresa } \\
\text { Feldaufgang }\end{array}$ \\
\hline $\begin{array}{l}11.03 .97 \\
10.04 .97\end{array}$ & $\begin{array}{l}\text { N-Düngung } \\
\text { Herbizideinsatz }\end{array}$ & $\begin{array}{l}70 \mathrm{~kg} \mathrm{~N} / \mathrm{ha} \text { als Kalkammonsalperter } \\
0,6 \mathrm{l} / \mathrm{ha} \text { Ralon Super }+0,6 \mathrm{l} / \mathrm{ha} \text { Starane } \\
(+1,0 \mathrm{l} / \mathrm{ha} \text { Oleo })\end{array}$ \\
\hline $\begin{array}{l}25.04 .97 \\
22.07 .97\end{array}$ & $\begin{array}{l}\text { Herbizideinsatz } \\
\text { Ernte }\end{array}$ & $\begin{array}{l}\text { 2,0 I/ha IPU } 500 \\
\text { Ernteparzellen mit der Sense }\end{array}$ \\
\hline
\end{tabular}


Tab. 58: Pflanzenbauliche Maßnahmen auf dem Standort Relliehausen für die Bearbeitungssysteme Lockerbodenwirtschaft (LBW) und Festboden-Mulchwirtschaft (FMW) in den Jahren 1995 bis 1997.

\begin{tabular}{|c|c|c|}
\hline $\begin{array}{l}\text { Datum } \\
25 . / 26 . \\
04.95\end{array}$ & $\begin{array}{l}\text { Maßnahme } \\
\text { Versuchsanlage }\end{array}$ & Beschreibung \\
\hline 26.04 .95 & $\begin{array}{l}\text { Saatbettbereitung/ } \\
\text { Aussaat }\end{array}$ & $\begin{array}{l}\text { Kreiseldrillkombination } \\
\text { Sommergerste, Sorte: Maresi }\end{array}$ \\
\hline 05.05 .95 & & Feldaufgang \\
\hline 24.05 .95 & $\mathrm{~N}$-Düngung & $46 \mathrm{~kg} \mathrm{~N} / \mathrm{ha}$ als Harnstoff \\
\hline 01.09 .95 & Ernte & Ernteparzellen mit der Sense \\
\hline 07.09 .95 & Stoppelbearbeitung & Scheibenegge, Tiefe: $7 \mathrm{~cm}$ \\
\hline 16.10 .95 & Stoppelbearbeitung & Kreiselegge, Tiefe $5 \mathrm{~cm}, 2 \times$ diagonal \\
\hline 31.10 .95 & Grundbodenbearbeitung & $\begin{array}{l}\text { LBW: Streichblechpflug, Tiefe: } 25 \mathrm{~cm} \\
\text { FMW: keine Bearbeitung }\end{array}$ \\
\hline 01.11 .95 & $\begin{array}{l}\text { Saatbettbereitung/ } \\
\text { Aussaat }\end{array}$ & $\begin{array}{l}\text { Kreiselegge + Walze/ } \\
\text { Winterweizen, Sorte: Zentos }\end{array}$ \\
\hline 12.11 .95 & & Feldaufgang \\
\hline 08.05 .96 & $\begin{array}{l}\text { N-Düngung/ } \\
\text { Herbizideinsatz/ } \\
\text { Halmverkürzer }\end{array}$ & $\begin{array}{l}73 \mathrm{~kg} \mathrm{~N} / \mathrm{ha} \text { als Ammonnitrat-Harnstoff- } \\
\text { lösung }+1,5 \mathrm{l} / \mathrm{ha} \text { Lumeton }+0,5 \mathrm{l} / \mathrm{ha} \\
\text { Chlorcholinchlorid }\end{array}$ \\
\hline 14.06 .96 & $\begin{array}{l}\text { N-Düngung/ } \\
\text { Fungizideinsatz/ } \\
\text { Wachstumsregler }\end{array}$ & $\begin{array}{l}46 \mathrm{~kg} \mathrm{~N} / \mathrm{ha} \text { als Harnstoff } \\
+0,7 \mathrm{l} / \mathrm{ha} \text { Opus Top } \\
+0,4 \mathrm{l} / \mathrm{ha} \text { Zenit }\end{array}$ \\
\hline 04.09 .96 & Ernte & Ernteparzellen mit der Sense \\
\hline 12.09 .96 & Stoppelbearbeitung & Scheibenegge, Tiefe $10 \mathrm{~cm}$ \\
\hline 18.09 .96 & Grundbodenbearbeitung & $\begin{array}{l}\text { LBW: Streichblechpflug, Tiefe: } 25 \mathrm{~cm} \\
\text { FMW: keine Bearbeitung }\end{array}$ \\
\hline 23.09 .96 & $\begin{array}{l}\text { Saatbettbereitung/ } \\
\text { Aussaat }\end{array}$ & $\begin{array}{l}\text { Kreiseldrillkombination, } \\
\text { Wintergerste, Sorte: Theresa }\end{array}$ \\
\hline 02.10 .96 & & Feldaufgang \\
\hline 12.03.97 & $\begin{array}{l}\text { N-Düngung/ } \\
\text { Herbizideisatz }\end{array}$ & $\begin{array}{l}72 \mathrm{~kg} \mathrm{~N} / \mathrm{ha} \text { als Ammonnitrat-Harnstoff- } \\
\text { lösung }+0,6 \mathrm{l} / \mathrm{ha} \text { Fenikan }+1,4 \text { I/ha IPU }\end{array}$ \\
\hline 15.05 .97 & $\begin{array}{l}\text { Fungizideinsatz/ } \\
\text { Wachstumsregler }\end{array}$ & $\begin{array}{l}0,5 \mathrm{I} / \text { ha Zera }+0,7 \mathrm{I} / \text { ha Juwel }+0,7 \mathrm{I} / \mathrm{ha} \\
\text { Chlorcholinchlorid }\end{array}$ \\
\hline 20.05 .97 & $\mathrm{~N}$-Düngung & $46 \mathrm{~kg} \mathrm{~N} / \mathrm{ha}$ als Harnstoff \\
\hline 0.07 .97 & Ernte & Ernteparzellen mit der Sense \\
\hline
\end{tabular}


Tab. 59: Pflanzenbauliche Maßnahmen auf dem Standort Großobringen für die Bearbeitungssysteme Lockerbodenwirtschaft tief (LBW tief) und Lockerbodenwirtschaft flach (LBW flach) in den Jahren 1995 bis 1997.

\begin{tabular}{|c|c|c|}
\hline $\begin{array}{l}\text { Datum } \\
23 . / 24 . \\
03.95\end{array}$ & $\begin{array}{l}\text { Maßnahme } \\
\text { Versuchsanlage }\end{array}$ & Beschreibung \\
\hline 27.03.95 & Saatbettbereitung & $\begin{array}{l}\text { mehrmaliges Grubbern der Varianten } \\
6 \times 5 t\end{array}$ \\
\hline 03.04 .95 & $\begin{array}{l}\text { Sattbettbereitung/ } \\
\text { Aussaat }\end{array}$ & $\begin{array}{l}\text { Kombinationsdrillmaschine } \\
\text { Sommergerste, Sorte: Maresi }\end{array}$ \\
\hline 13.04 .95 & Herbizideinsatz & $\begin{array}{l}\text { Feldaufgang } \\
0,7 \mathrm{l} / \text { ha Starane }+25 \mathrm{~g} / \text { ha Pointer }\end{array}$ \\
\hline 29.05 .95 & Herbizideinsatz & $0,7 \mathrm{I} /$ ha M-Fluid A \\
\hline 14.08.95 & Ernte & Ernteparzellen mit der Sense \\
\hline 16.08.95 & Stoppelbearbeitung & Schwergrubber, Tiefe: $10 \mathrm{~cm}$ \\
\hline 08.09 .95 & Grundbodenbearbeitung & $\begin{array}{l}\text { LBW tief: Spatenmaschine, Tiefe: } 25 \mathrm{~cm} \\
\text { LBW flach: Spatemaschine, Tiefe: } 12 \mathrm{~cm}\end{array}$ \\
\hline 16.10 .95 & $\begin{array}{l}\text { Saatbettbereitung/ } \\
\text { Aussaat }\end{array}$ & $\begin{array}{l}\text { Kombinationsdrillmaschine } \\
\text { Winterweizen, Sorte: Aron }\end{array}$ \\
\hline 28.10 .95 & & Feldaufgang \\
\hline 23.04 .96 & Striegeln & Striegel \\
\hline 27.04 .96 & N-Düngung & $81 \mathrm{~kg} \mathrm{~N} / \mathrm{ha}$ als Kalkammonsalperter \\
\hline 14.06.96 & N-Düngung & $\begin{array}{l}40 \mathrm{~kg} \mathrm{~N} / \mathrm{ha} \text { als Ammonnitrat-Harnstoff- } \\
\text { lösung }\end{array}$ \\
\hline $\begin{array}{l}23.08 .96 \\
10.09 .96\end{array}$ & $\begin{array}{l}\text { Ernte } \\
\text { Abschlägeln der } \\
\text { Stoppeln }\end{array}$ & $\begin{array}{l}\text { Ernteparzellen mit der Sense } \\
\text { Schlägelhäcksler }\end{array}$ \\
\hline 12.09.96 & Stoppelbearbeitung & Scheibenegge, Tiefe: $7 \mathrm{~cm}$ \\
\hline 20.09 .96 & Grundbodenbearbeitung & $\begin{array}{l}\text { LBW tief: Spatenmaschine, Tiefe: } 25 \mathrm{~cm} \\
\text { LBW flach: Spatemaschine, Tiefe: } 12 \mathrm{~cm}\end{array}$ \\
\hline 01.10 .96 & $\begin{array}{l}\text { Sattbettbereitung/ } \\
\text { Aussaat }\end{array}$ & $\begin{array}{l}\text { Kombinationdrillmaschine } \\
\text { Wintergerste, Sorte: Grete }\end{array}$ \\
\hline 10.10 .96 & & Feldaufgang \\
\hline 04.11 .96 & N-Düngung & $30 \mathrm{kgN} / \mathrm{ha}$ als Ammoniumsulfat \\
\hline 07.03 .97 & N-Düngung & $54 \mathrm{kgN} / \mathrm{ha}$ als Kalkammonsalpeter \\
\hline 02.04 .97 & Herbizideinsatz & $0,7 \mathrm{l} /$ ha Starane $+25 \mathrm{~g} /$ ha Pointer \\
\hline 06.05 .97 & N-Düngung & $\begin{array}{l}72 \mathrm{kgN} / \mathrm{ha} \text { als Ammonnitrat-Harnstoff- } \\
\text { lösung }\end{array}$ \\
\hline 12.05 .97 & Herbizideinsatz & $1,0 \mathrm{l} / \mathrm{ha}$ Starane $+25 \mathrm{~g} / \mathrm{ha}$ Pointer \\
\hline
\end{tabular}




\section{Dank}

Mein herzlichster Dank gilt meiner lieben Anke und meinen Kindern Mathis und Lena. Sie zeigten mir immer wieder mit ihrem Lachen, was für mich wirklich wichtig ist.

Ganz besonderer Dank soll meinen Eltern zukommen. Sie unterstützten mich wo immer sie konnten mit Rat und Tat.

Herrn Prof. Dr. Wilfried Ehlers danke ich für die Bereitstellung des interessanten Themas, für seine Unterstützung bei der Planung, Durchführung und Auswertung der Feldversuche und für seine wertvolle Hilfe bei der Fertigstellung der Arbeit.

Herrn Prof. Dr. Michael Runge danke ich für die Übernahme des Korreferats.

Herrn Dr. habil. Dietrich Werner von der TLL Jena bin ich sehr dankbar für seine wertvolle Unterstützung bei den Feldversuchen und für die Bereitstellung seiner Meßergebnisse.

Mein ganz besonderer Dank gilt Herrn Klaus George. Er übernahm die Führung der Feldversuche auf dem Standort Großobringen.

Allen Mitarbeitern und Mitarbeiterinnen „unseres“ DFG-Forschungsvorhabens sei an dieser Stelle für ihre Hilfe gedankt.

Mein aufrichtiger Dank gilt allen Kräften des Institutes für Pflanzenbau und Pflanzenzüchtung, die durch ihre sorgfältige und ausdauernde Arbeit zum Gelingen meiner Arbeit beigetragen haben. Besonders erwähnt werden soll Frau Anita Bartlitz. Ohne ihren Einfluß hätte die Datenerfassung leicht zu einem Chaos werden können.

Meinen Kolleginnen und Kollegen Annette Muhs, Barbara Hohlmann, Dr. Thomas Forstreuter und Helmut Kimpel-Freund danke ich herzlich für die freundschaftiche Zusammenarbeit und die stets aufschlußreiche und erfrischende Diskussion privater und innerbetrieblicher Zusammenhänge. 
Frau Dr. Friederike de Mol danke ich herzlich für die kritische Durchsicht des Textes und das so manches Mal gewährte Obdach.

Herrn Dr. Stefan Fenner danke ich für die Einführung in die Geheimnisse der Gasdiffusion und der ungesättigten Wasserleitfähigkeit in Böden.

Herrn Christian Sirie und seinen Mitarbeitern vom Versuchsgut Relliehausen sei für die sorgfältige und zuverlässige Durchführung aller notwendigen Arbeiten auf dem Standort Relliehausen gedankt.

Der Firma Orenstein und Koppel gilt mein Dank für die unkomplizierte Bereitstellung der Radlader.

Der Deutschen Forschungsgemeinschaft danke ich für die finanzielle Unterstützung. 


\section{Lebenslauf}

\section{Persönliche Daten:}

Name:

Geburtstag:

Karl-Theodor Mähner

Geburtsort:

Staatsangehörigkeit:

28.10.1963

Hamm/ Westfalen

deutsch

Familienstand:

ledig, zwei Kinder

\section{Schulausbildung:}

1970 bis 1974

Grundschule in Hamm / Westfalen

1974 bis 1984

Mai 1984

Gymnasium in Hamm / Westfalen

Allgemeine Hochschulreife

1984 bis 1985

Grundwehrdienst in Flensburg

\section{Berufsausbildung:}

Ausbildung zum staatlich geprüften landwirtschaftlichen Gesellen:

1985 bis1986 erstes Lehrjahr auf dem Hof Friedrich Wilms-Schulze Kump in Hamm/Westfalen

(120 Hektar Ackerbau, Schweinemast und Ferkelaufzucht)

1986 bis1987 zweites Lehrjahr auf dem Hof Franz Kaiser in Süddinker / Westfalen

(35 Hektar Weide / Futterbau, 10 Hektar Ackerbau und 28 Milchkühe mit Kälberaufzucht)

Juni 1987 Abschlußprüfung an der Landwirtschaftskammer Westfalen/Lippe

\section{Hochschulausbildung:}

1987 bis 1991 Grundstudium Agrarwissenschaften an der Universität Göttingen

1991 bis 1994 Hauptstudium Agrarwissenschaften (Fachrichtung

Pflanzenbau) an der Universität Göttingen

Diplomarbeit: Die Auswirkungen der Bodenbearbeitung auf die Evaporation eines syrischen Vertisols

Erfolgreicher Abschluß des Studiums zum Dipl.-Ing. agr 


\section{Berufstätigkeit:}

1995 bis 1998 Wissenschaftlicher Angestellter der Universität Göttingen, Institut für Pflanzenbau und Pflanzenzüchtung

\section{Auslandspraktika:}

Mai - Juli 1989 Aufenthalt in Syrien und Ägypten. Praktikum Trockenfeldbau Juli - Sept.1990 Aufenthalt in Costa Rica. Praktikum Kaffeeanbau.

Juni - Aug.1992 Aufenthalt in Malaysia/Borneo. Praktikum Tee- und Ölpalmenanbau.

Okt.'92-April'93 Aufenthalt in Syrien. Wissenschaftlicher Angestellter am International Center For Agricultural Research In Dry Areas (ICARDA). 